\title{
The Effects of Compulsory Schooling Laws on Teenage Marriage and Births in Turkey
}

\author{
Murat G. Kirdar \\ Department of Economics, Boğaziçi University, 34342 İstanbul, Turkey \\ E-mail: murat.kirdar@boun.edu.tr
}

\section{Meltem Dayığlu}

Department of Economics, Middle East Technical University, 06800, Ankara, Turkey

E-mail: $\underline{\text { dmeltem@ @etu.edu.tr }}$

\author{
İsmet Koç
}

Institute of Population Studies, Hacettepe University, 06100, Ankara, Turkey

E-mail: iskoc@hacettepe.edu.tr 


\title{
The Effects of Compulsory Schooling Laws
}

\section{on Teenage Marriage and Births in Turkey*}

\author{
Murat G. Kırdar \\ Department of Economics, Boğaziçi University, 34342 İstanbul, Turkey \\ e-mail: murat.kirdar@boun.edu.tr
}

Meltem Dayığglu

Department of Economics, Middle East Technical University, 06800 Ankara, Turkey

e-mail:dmeltem@metu.edu.tr

\begin{abstract}
İsmet Koç
Institute of Population Studies, Hacettepe University, 06100 Ankara, Turkey

e-mail: iskoc@hacettepe.edu.tr
\end{abstract}

* We would like to thank Abdurrahman Aydemir, Anna Batyra, Matias Catteneo, Alper Dinçer, Ronald Lee, Çağlar Özden, Steffen Reinhold, Nicole Schneeweis, İnsan Tunalı, and the participants at the Society of Labor Economists, Population Association of America, Economic Research Forum, Society of Economic Dynamics, Middle East Economic Association, and Koç University ERF Conference on Education, Health, and Worker Productivity conferences and the Econometric Society World Congress as well as the seminar participants at the University of California-Berkeley and Ryerson and Özyeğin Universities for their helpful comments and suggestions. Financial support from the Turkish Scientific and Technological Council Grant 108K251 (2008) is gratefully acknowledged. All errors are our own. 


\begin{abstract}
This paper estimates the impact of the extension of compulsory schooling from 5 to 8 years in Turkey_which increased women's schooling by more than a year-on marriage and birth outcomes of teenage women. We employ a regression discontinuity design, where we compare month-year of birth cohorts of women. The increased compulsory schooling years reduce the probability of teenage marriage by age 16 and first-births by age 17 substantially. However, these effects dissapear after ages 17 and 18 for marriage and first-births, respectively. Our results indicate strong incarceration effects of the policy on marriage and birth outcomes during the newly mandated compulsory schooling years as well as human capital effects on the time to marriage. However, the human capital effects on the probability of being ever-married vanish after a couple of years the students become free to leave school.
\end{abstract}

Keywords: Teenage marriage; births; education; compulsory schooling; regressiondiscontinuity. 


\section{Introduction}

Teenage marriage and births remain at significant levels in Turkey. According to the 2013 Turkish Demographic and Health Survey (TDHS), of the population of 25- to 49-year-old women, 41 percent were married by age 20,22 percent were married by age 18 , and four percent were married by age 15. The percentage having their first-birth in their teenage years is also high: 26 percent gave birth to their first child by age 20. Adolescent marriage is widespread in other parts of the world as well. According to the International Center for Research on Women, 700 million women alive today were married by age 18; moreover, adolescent marriage occurs in both low and high income countries and across different regions, religions, and cultures. ${ }^{1}$ There is also a strong correlation between teenage marriage and education in several developing countries. In Turkey, among women aged 25 to 49 in 2013, while the median age of marriage was 18.9 for those with no school degree, it was 24.6 for those with a high school diploma or above. However, does this imply that an increase in women's educational attainment decreases teenage marriage and births?

In this study, we examine the effects of an increase in women's educational attainment-through the implementation of a longer compulsory schooling duration policy—on age at marriage, age at firstbirth, the time to first-birth after marriage, and the number of children ever born. We also investigate the channels through which the new compulsory schooling policy affects teenage marriage and births. One channel is the incarceration effect (also called the institution effect), which takes place because

\footnotetext{
${ }^{1}$ Among women of 20-24 years of age, the percentage of those marrying before age 18 was 75 in Niger, 66 in Bangladesh, 56 in Mozambique, 47 in India, and 41 in Ethiopia according to the most recent Demographic and Health Survey (DHS) statistics for these countries (International Center for Research on Women, 2015).
} 
schooling and marriage are incompatible events. The second channel is the human capital effect, which refers to the effects of education through its effects on wages, preferences, and knowledge.

For this purpose, we use a major education reform in Turkey in 1997 that extended compulsory schooling from 5 to 8 years. An important distinguishing characteristic of our study is the strength of the exogenous variation in schooling due to the length of the extension of compulsory schooling in Turkey (an additional three years) and the high percentage of children whose behavior is affected. In fact, the number of students in grades 1 to 8 increased by 15 percent (from 9 million to 10.5 million) in the three-year period following the implementation of the policy, compared to a 1-percent decline in the preceding three-year period (Turkish Ministry of Education, 2011). Kirdar et al. (2015) report that the policy increases women's completed years of schooling by more than a year.

In many developing countries, teenage marriage and births are associated with several adverse outcomes, including poorer mother and child health (see, for instance, Alam [2000] for Bangladesh, Raj et al. [2009, 2010] for India), worse educational outcomes (see, for instance, Lloyd and Mensch [2008] for sub-Saharan Africa), and a higher probability of domestic violence (UNICEF, 2005). There is also evidence for causal effects of early marriage; for instance, Field and Ambrus (2008) find a positive effect of delayed age at marriage on years of schooling in Bangladesh. In Turkey, for mothers younger than 20 , the neonatal mortality rate was almost twice as high and the perinatal mortality rate was nearly three times that for mothers aged 20 to 29 during the period from 1998 to 2008 (2008 TDHS). In a study conducted in eastern Turkey, where teenage marriage is especially common, Edirne et al. (2010) find that teenage mothers have less education and are more likely to face domestic violence, and their births have a higher risk of preterm delivery and low birth weight.

In conservative countries, where giving birth out of wedlock is socially condemned and therefore rare, mandating that teens stay in school for a longer time is a potentially important intervention on 
childbearing because longer schooling delays the entry of girls into the marriage market. An important characteristic of the transition to motherhood of women in Turkey is that almost all are married at the time of giving birth. The sociology literature reports a rigid sequence of events for completion of education, marriage and the birth of the first child in other countries as well (Blossfeld and De Rose, 1992; Marini, 1984). A key fact in this sequence of events is that the gap between the age at marriage and first-birth is quite narrow in Turkey. Given this narrow gap and the fact that marriage and schooling are generally incompatible events, a change in the timing of marriage due to increased schooling would directly translate into a change in the timing of fertility. ${ }^{2}$

The data in this study come from the 2008 and 2013 Turkish Demographic and Health Surveys, which are nationally representative and contain detailed information on transitions to marriage and fertility. Our sample includes both ever-married and never-married women. The structure of the data is suitable for a regression discontinuity design (RDD) in estimating the effects of the compulsory schooling policy. We use the variation in policy exposure across month-year of birth cohorts as well as year of birth cohorts.

In this study, we estimate the effect of the compulsory schooling policy rather than use it as an instrument for women's education because the change in compulsory schooling in Turkey

\footnotetext{
${ }^{2}$ The fraction of women who get married by age 18 in Turkey is very similar to that in many other Muslim-majority countries (including the largest ones), where a rigid sequence of education, marriage, and fertility also exists. The percentage of 20-24 year old women who are married by age 18 in Turkey (at 15 percent) is somewhat lower than that in Pakistan (at 21 percent) but very similar to that in Egypt and in Iran (at 17 percent), in Morocco (at 16 percent), in Indonesia and Syria (at 14 percent), in Kyrgyzstan and in Tajikistan (at 12 percent), and in Azerbaijan (at 11 percent) (UNICEF, 2016). Hence, the findings of this paper can be informative for other countries as well.
} 
substantially alters both women's and men's distribution of education. This could significantly alter both the marriage market and labor market opportunities for women. As a result, the exclusion restriction assumption - that the policy affects marriage and childbearing outcomes of a woman through the change in her education only - is likely to be violated.

Our results indicate that the extension of compulsory schooling reduces the probabilities of teenage marriage and births — but only upto a certain age. The probability of marriage by age 16 and, as a result of the rigid sequence of marriage and childbearing, the probability of giving birth by age 17 are both reduced by about 50 percent. However, the policy also increases the probability of marriage at age 17 conditional on not getting married until that age. Due to this catching-up effect shortly after the girls are out of school, the probability of being ever-married reverts to its pre-policy level after age 17. We find no evidence of a policy effect on the time to first-birth after a woman is married. Consequently, the policy effect on ever giving birth also disappears after age 18. In essence, we find strong evidence for the incarceration effect whereas the human capital effect is short-lived.

The outline of the paper is as follows. Section 2 places our study in the relevant literature. Section 3 provides background information on marriage and fertility behavior and the education system in Turkey. Section 4 presents the conceptual framework that guides the interpretation of our results. Data and descriptive statistics are given in Section 5, and the identification strategy and estimation in Section 6. Section 7 presents the results, while Section 8 provides an overall discussion on the results. Section 9 concludes.

\section{Relevant Literature}

In the related literature, most studies in the international context focus on the effect of education on teenage fertility rather than marriage as many of these studies are for countries where there is no rigid 
sequence of marriage and fertility. In terms of marriage outcomes, Lefgren and McIntyre (2006) find that education has no effect on whether a woman in the US ever gets married; however, Brien and Lillard (1994) and Skirbekk et al. (2004) find that education delays the age at marriage in Malaysia and Sweden, respectively. The available evidence on the causal effect of education on teenage fertility is mixed. While Black et al. (2008) for the US and Norway, Silles (2011) for Great Britain and Ireland, Ozier (2011) for Kenya, Grönqvist and Hall (2013) for Sweden, and DeCicca and Krashinsky (2015) for Canada show that teenage pregnancy is reduced by education, McCrary and Royer (2011) find no such effect in the US in an influential study.

A previous working-paper version of this paper (Kirdar et al., 2009) is first to study the causal impact of schooling on marriage and birth outcomes in the Turkish context. We improve upon the working paper by using the 2013 TDHS, which has only recently become available and which allows us to separate the policy effect from the time trends in the outcome variables much more convincingly within RDD — thereby leading to more robust and precise results_-for the following reasons. First, the number of data points on the right-hand-side of the cut-off significantly increases when we use the 2013 THDS due to the rather recent implementation of the policy. Second, we use month-year as well as year of birth as the running variable in this version, which not only increases the number of data points on each side of the cut-off but also allows zooming in around the cut-off more. (Since month-year of birth information is available only for the ever-married sample in the 2008 THDS, it was not used in Kirdar et al. [2009].)

Kirdar et al. (2009) is followed by Dincer et al. (2014) and Gunes (2013), which also examine the effect of schooling on certain marriage and birth outcomes in the Turkish context using the same policy change. Our paper substantially differs from the latter two papers in terms of content, methodology, and - most importantly — findings. In terms of content, the key differences of our paper 
are that it separates the level (quantum) effects of the policy from the timing (tempo) effects and it disentangles the human-capital and incarceration effects of increased schooling on marriage and birth outcomes.

Our results are starkly different from the results of these two papers. Gunes (2015) claims that the policy effect on fertility persists beyond age 18, in fact up to age 22; while Dincer et al. (2014) find that a 10 -percentage point increase in the proportion completing 8 years of schooling decreases the number of children between 0.11 and 0.16 - for women between ages 18 to 22 . We, however, find no policy effect on the number of children born to a woman beyond age 18 - although we do find an effect by age 18 . This difference, we assert, stems from our cleaner identification methodology.

In terms of methodology, Dincer et al. uses an instrument based on geographical variation in the policy intensity. However, in the Turkish context, the policy intensity is far from being "as good as" randomly assigned across locations — which raises concerns about the validity of this instrument. Gunes (2015), on the other hand, uses a difference-in-differences methodology. However, the trends in marriage and birth outcomes across regions exhibit substantial variation in Turkey (where level differences across regions are also substantial); thus, the common trend assumption in the differencein-difference methodology simply does not hold in this context. Our paper goes beyond these papers in terms of internal validity by using RDD and by utilizing richer data. Lee and Lemieux (2010) argue that RDD is potentially more credible than other quasi-experimental approaches. Lee (2008) shows that there is no need to assume that the treatment variable is "as good as" randomly assigned in RDD because of invidivuals' imprecise control over the assigment variable. In this sense, Lee argues that 
RDD is much closer to randomized experiments (the "gold standard" of program evaluation methods) than other quasi-experimental methods. ${ }^{3}$

Another related study that uses the same reform in the Turkish context is Gulesci and Meyersson (2013) — which also implements RDD to examine the reform's effect on religiosity and women's empowerment. Among the myriad of outcomes examined in this paper, one is age at marriage; and they find no policy effect on this variable. That they find an imprecise small effect is presumably not surprising as they do not account for the fuzziness in the treatment variable around the cut-off point and they use data-demanding non-parametric RDD with a relatively small sample size. In essence, while we find strong and robust policy effects on teenage marriage and fertility — unlike Gulesci and Meyersson - we also show that these effects do not persist beyond a certain age - unlike Dincer et al. (2014) and Gunes (2013).

\section{Background Information}

\subsection{Marriage and Fertility}

Despite the rise in non-marital unions in the West, marriage as a social institution remains strong in Turkey. Almost all women marry by age 49 and 98 percent give birth at least once. Moreover, divorce is an unlikely event, with its rate estimated at less than 2 percent among women of 15-49 years of age (TDHS-2013). Giving birth out of wedlock is even rarer; according to TDHS-2013 data, almost all births are given by married women. Age at first-birth is also closely linked to age at marriage; the

\footnotetext{
${ }^{3}$ McCrary and Royer (2011), Ozier (2015), and Silles (2011) also use RDD to examine the effect of schooling on teenage fertility in other contexts.
} 
lapse of time between marriage and first-birth is on average 1.6 years. The average fertility rate currently stands at 2.26 births per woman (TDHS-2013).

Marriage occurs early in life. The median age at first marriage is 21.0 years in TDHS-2013. However, age at marriage has been increasing in Turkey. According to TDHS-2013, while the median age for marriage is 20.2 for the $45-49$ age group, it is 22.0 for the $25-29$ age group; in fact, from 1993 to 2013, the median age at marriage has increased by almost two years. Despite the rise over time in age at marriage, the incidence of teenage marriage remains high. Of the women aged 20-24 in TDHS2013, 29 percent are married before age 20 and 15 percent are married before age 18 .

As a result of their early marriage, Turkish women give their first birth at an early age as well: the median age at first-birth for 25- to 49-year-old women is 22.9 (TDHS-2013). Age at first-birth has also increased over time with the rising age at marriage. Nonetheless, the incidence of first-birth during teenage years remains high even among the younger generations. Of the women aged 20-24, 17 percent had given birth before age 20 and 6 percent before age 18 (TDHS-2013).

The Civil Code prohibits marriage before age 17; however, a marriage can still happen through a religious ceremony before this age. Indeed, 2.5 percent of 16-year-old women in 2013 were married. Many couples have both a religious and a civil marriage ceremony; however, the percentage of women who have a religious marriage only is significant, especially among teenage women. The TDHS-1993 and TDHS-2013 data indicate that the percentage of 15- to 19-year-old married women who had only a religious marriage ceremony was 33 percent in 1993, and dropped only to 22 percent by 2013 . 


\subsection{New Compulsory Schooling Policy}

Before the change in the basic education law, the education system in Turkey consisted of five years of primary, three years of lower secondary, and three years of high school. The first tier was compulsory. In 1997, the Ministry of National Education (MONE) increased compulsory education from five to eight years by combining the first two tiers through amendments made to the Basic Education Law (no. 4306, dated 16 August 1997). The new law covered all children who did not already hold a primary school diploma at the beginning of the 1997-98 school year, without making exceptions for any group - although non-compliance was common both before and after the policy.

Although improving the enrollment at the lower secondary level was a long-established goal, the exact timing of the implementation of the new policy had to do with the political developments of that time. The secular government that came to power in 1997 wanted to prevent children from enrolling in religious schools at an early age. This policy reform would delay this by three years. It is important to note that the timing of the policy was unexpected and its impact on schooling outcomes was realized in a short period of time.

Before the enactment of the law, the net school enrollment rate was 89.4 percent in five-year compulsory schooling, 52.8 percent in lower secondary, and 38.5 percent in upper secondary schooling (Turkish Statistical Institute, 2012). Figure A1 in the Appendix displays how the number of students in grades 1 to 8 changed over the school years for urban and rural areas. From the 199798 school year to the 2000-01 school year, the number of students in grades 1-8 in urban areas increased by 13.7 percent compared with a 1.8-percent increase in the preceding three years, and the number of students in grades 1-8 in rural areas increased by 20 percent compared with a 7-percent fall in the preceding three years. Kırdar et al. (2015) report significant spillover effects to high school grade levels; in fact, the number of students in high school in urban areas increased by 27 percent 
from the 2000-01 school year to the 2003-04 school year, compared with a 10.5-percent rise in the previous three-year period.

To accommodate this substantial increase in the number of students, the government implemented a number of initiatives such as expanding the number of classrooms in existing schools, bussing an additional half a million (mostly rural) children to nearby schools, and constructing almost 600 boarding schools in more remote areas (Kurdar et al. [2015]). Accordingly, the share of MONE in the public investment budget soared from 15 percent in 1997 to 37.3 percent in 1998. As a result of this substantial investment in schooling infrastructure, schooling quality as measured by student-toteacher and student-to-classroom ratios remained relatively constant (Kırdar et al. [2015]).

Since the new law covered children who had completed grade four or lower at the end of the 199697 school year, compulsory schooling became eight years for all students who had started the first grade as of September 1993, but it was five years for those who had started earlier. (There is virtually no grade repetition at this schooling level in Turkey.) The education law stipulates that children start school in the September of the year in which they complete their 72nd month (Resmi Gazete, no. 21308, dated 7 August 1992). Therefore, children born in or after January 1987 were affected by the policy. However, the treatment status of the birth cohorts right around the point of discontinuity is fuzzy. This is mainly because not all children start school at age six in Turkey. Children who were born before January 1987 but started school later than age 6, which is frequent in Turkey, were bound by the policy. On the contrary, children who were born after January 1987 but started school earlier than the normal school start age (and thus had a primary school diploma by the time of the policy) were exempt from the policy. 


\section{Conceptual Framework}

There are two main channels through which education influences marriage: the institution effect and the human capital effect. According to the institution effect, schooling and marriage are incompatible events. Thornton et al. (1995) point out three main reasons for this: i) students are not prepared for adult roles, ii) school requires a lot of time, iii) financial independence is needed for marriage. This argument is consistent with Becker's specialization hypothesis (Becker, 1973) in that the gains from specialization cannot be realized until after schooling is completed: women would not have sufficient time for home production and men would not have sufficient income. The institution effect is also referred to as the "incarceration effect" in the economics literature.

According to the human capital effect, even after schooling is completed, increased education has a bearing on marriage choices. A particular channel through which this happens is market work. With increased schooling, the opportunity cost of marriage and raising children increases for women. This is the channel that is emphasized in the work of Becker and in the economics literature in general. Another channel for the human capital effect is a change in marriage preferences with higher schooling levels. Axinn and Barber (2001) discuss "ideational theories", which explain how schooling changes fertility preferences. An example is that schooling could increase consumption aspirations, thus increasing the costs of childbearing and decreasing the demand for children. Similar arguments could be applied to marriage; hence, a longer education period could change marriage preferences.

Education affects fertility decisions through a number of channels. ${ }^{4}$ First, the effect of education on marriage translates into an effect on fertility in the rigid sequence of marriage and fertility decisions

\footnotetext{
${ }^{4}$ Glewwe (2002) reviews the literature on the relationship between schooling and marriage and fertility in developing countries.
} 
in Turkey, as well as in several other countries. However, there are additional human capital effects of education on fertility. First, a higher level of education for women increases the opportunity cost of raising children by increasing their market wage rate (Willis, 1973). This would decrease the desired number of children. ${ }^{5}$ In addition, Rosenzweig and Schultz (1989) show that education increases the effective contraceptive use of women. Education can provide better knowledge of contraceptive methods via curricula or develop the ability to acquire information about them. The second channel was formalized by Grossman (1972), for which Glewwe (1999) finds empirical evidence in Morocco. Finally, as claimed by Mason (1986), higher bargaining power in fertility decisions for more educated women is another possible channel through which education influences fertility decisions.

\section{Data and Descriptive Statistics}

The data we employ come from the 2008 and 2013 rounds of the Turkish Demographic and Health Survey (TDHS), which is nationally representative. The target population in TDHS is women at the reproductive age (15-49). The sample includes both ever-married and never-married women. The surveys include information on the timing of marriage and the timing of first-birth for ever-married women, as well as information on schooling attainment in the form of the highest grade completed. These three pieces of information are the key outcome variables in this study. We also use information on location of birth in our analyses - in terms of rural vs. urban residence and the geographical region of residence (12 NUTS-1 level regions).

\footnotetext{
${ }^{5}$ This is on condition that the substitution effect dominates the income effect (higher demand for children due to a higher level of income). The income effect would be stronger if there is positive assortative mating; in other words, women who acquire more schooling marry more educated men with higher incomes.
} 
The 2013 survey includes information on the month as well as the year of birth for all women, whereas the 2008 survey includes this information only for ever-married women. Therefore, we use two separate samples. In the first sample, we pool the 2008 and 2013 surveys and define cohorts according to year of birth. In addition, we drop the 1986 and 1987 birth cohorts from the sample due to the fuzziness in the treatment status of these two cohorts right around the cut-off (as explained in Section 3.2) and call this gap the "donut-hole" around the cut-off in the rest of the paper. ${ }^{6}$ Since the surveys draw among women aged 15-49, this sample includes women—ever married or never married—born between 1959 and 1998, except for the 1986 and 1987 birth cohorts. We call this sample (A) — the full sample. In the second sample, we use the 2013 survey only and define cohorts according to month-year of birth. We further disaggregate this sample according to the width of the time window taken around the donut-hole. Sample (B1) takes 4-year intervals around the donut-hole (1982-85 and 1988-91 birth cohorts), sample (B2) 7-year intervals around the donut-hole (1979-85 and 1988-94 birth-cohorts), and sample (B3) 10-year intervals (1976-85 and 1988-97 birth-cohorts). In samples (B1) to (B3), month of birth is an additional variable. Basic descriptive statistics on person-level characteristics are displayed for sample (A) in Table A1 and for samples (B1) to (B3) in Table A2 of Appendix A.

Using the information on age at marriage and age at first birth, we construct histories of ever-married status and ever-given-birth status until age 19 or until the current age at the time of the survey, whichever comes earlier. This means that the number of birth cohorts on the right-hand side of the discontinuity changes by age. For instance, all birth cohorts until 1998 contribute to the sample for age 15, whereas only birth cohorts until 1994 contribute to the sample for age 19 (as the latest survey is in 2013). Hence, the number of observations fall after age 15 with age in samples (A) and (B3) but

\footnotetext{
${ }^{6}$ The reason for this is discussed in detail in the next section on identification strategy.
} 
not for samples (B1) and (B2) because both the 4-year and 7-year intervals around the donut-hole end before 1994.

The effects of the education reform on three different schooling outcomes-eight-year basic education completion, high school completion, and average years of schooling - are illustrated in Figure 1, where panel (A) uses sample (A) with year of birth as the running variable and panel (B) uses sample (B2) with month-year of birth as the running variable. Panels (A1) and (B1) indicate a substantial increase in the proportion of women who complete the $8^{\text {th }}$ grade with the policy, from about 40 percent before the policy to almost 70 percent after the policy. Another interesting feature of Figure 1 is the rise in the percentage of women who complete the $11^{\text {th }}$ grade (high school) following the policy implementation, displayed in panels (A2) and (B2). In other words, the new policy seems to affect grade completion rates well beyond the new compulsory schooling levels. Panels (A3) and (B3) indicate an important jump in the total years of schooling between the 1985 and 1988 birth cohorts.

Changes in the percentage of women who are ever married at each age from 14 to 19 are displayed in Figure 2 for the year of birth cohorts in sample (A) and in Figure 3 for the month-year of birth cohorts in sample (B2). The discontinuity in the percentage of ever married at the time of the policy is visible at ages 14,15 , and 16 in both figures. On the other hand, the graphs suggest no discontinuity in the percentage of ever married at or above age 17. Similarly, the way the percentage of women who have ever given birth at each age from 14 to 19 changes is given in Figure 4 for the year of birth cohorts in sample (A) and in Figure 5 for the month-year of birth cohorts in sample (B2). In this case, the jumps at the cut-off are more visible at ages 15, 16, and 17. Given the fact that the gap between the time of first-birth and the time of marriage is just over a year, the discontinuities in Figures 3 and 5 are consistent with the discontinuities in Figures 2 and 4, respectively. There are also strong time 
trends in Figures 2 to 5, but especially in Figures 2 and 4 with a wider time window. Therefore, in the identification strategy, it will be critical to disentangle the policy effects on marriage and birth outcomes from these secular time trends.

\section{Identification Strategy and Estimation}

We use the variation in the years of compulsory schooling across different month-year of birth cohorts, as well as that across different year of birth cohorts, to identify the causal impact of the new compulsory schooling policy. The structure of our data, illustrated in Figures 1 to 5, fits a regressiondiscontinuity design, as there is a discontinuous jump in the outcome variables at the time of the policy, and the relationship between the outcome variables and the covariate that determines the timing of the jump (running variable) can be taken to be roughly continuous.

The usual way to handle the fuzziness in the data around the discontinuity is to apply a fuzzy regression discontinuity design — which is essentially a 2SLS estimation where the policy dummy is used as an instrumental variable. However, using an instrumental variable requires that the exclusion restriction assumption holds - in other words, the instrument has no direct effect on potential outcomes. In our context, however, the exclusion restriction assumption is not plausible because the compulsory schooling policy in Turkey makes substantial changes in both women's and men's distribution of education. This change could significantly alter both the marriage market and labor market opportunities for women. Moreover, since the policy changes not only the schooling of women but also potentially that of their husbands, we could be measuring the joint effect of the rise in the schooling of both spouses. As a result, the exclusion restriction assumption - that the policy affects marriage and childbearing outcomes of a woman through a change in her education only - is 
likely to be violated. ${ }^{7}$ Hence, we measure the policy effect rather than attempt to measure the effect of an increase in women's education.

Since we estimate the policy effect on potential outcomes (rather than the effect of schooling), we have to use sharp RDD. Hence, we remove the fuzziness from our data based on an exogenous covariate by excluding the 1986 and 1987 birth cohorts (the donut-hole). ${ }^{8}$ Donut-hole RD specifications, which exclude observations in the immediate vicinity of the cut-off, have recently been used in a number of studies (see, for instance, Almond et al. [2011], Bajari et al. [2011], Barreca et al. [2011], Card and Guiliano [2014], Fukishima et al. [2016], Hoxby and Bulman [2016]). ${ }^{9}$ For instance, Barreca et al. [2011] find that the previous estimates by Almond et al. (2010) on the effect of crossing the very-low-birth-weight threshold on infant mortality falls by more than 50 percent once the observations in the immediate vicinity of the threshold are excluded. ${ }^{10}$ While all RD designs require extrapolation, the level of extrapolation is higher in donut-hole $\mathrm{RD}$ designs, which requires that we extrapolate the time trends within the donut-hole until the cut-off point. This extrapolation

\footnotetext{
${ }^{7}$ Angrist et al. (1996) discuss the factors, like the fraction of compliers with the policy, which would determine the importance of such biases.
}

\footnotetext{
${ }^{8}$ Were we to estimate, instead, the effect of education using a 2SLS analysis, this exclusion would not be needed because in that case - within a Wald-estimator interpretation — both the numerator on the policy effect on marriage/first-birth and the denominator on the policy effect on education would be adjusted by the fuzzy treatment in the same way. In fact, as shown by Hahn et al. [2001], the fuzzy regression discontinuity design-which is equivalent to a two-stage least squares estimation - addresses this problem using the random assignment of the instrumental variable.

${ }^{9}$ In most of these studies, the reason for the exclusion of observations near the threshold is manipulation of the running variable around the threshold by the agents.

${ }^{10}$ Cattaneo et al. (2017) discuss the donut-hole RD estimation in their recent review chapter.
} 
within the donut-hole makes the experimentation with different functional forms for the time trend even more important in this study. We also assess the sensitivity of our findings to the width of the donut-hole in robustness checks.

Since we use a donut-role RDD, we find using global polynomial methods rather than local polynomial methods a more natural initial approach as the latter typically uses few observations around the cut-off. At the same time, we experiment with several bandwidths (time windows), as explained in the Data Section, by gradually zooming in around the cut-off. In other words, we show the robustness of our findings under various bandwidths, rather than choosing an optimal bandwidth choice as in the local polynomial approach.

Our data-generating process can be written as

$$
\begin{aligned}
& \mathrm{E}\left(\mathrm{Y}_{0 \mathrm{i}} \mid \mathrm{x}_{\mathrm{i}}\right)=f_{0}\left(\mathrm{x}_{\mathrm{i}}^{\prime}\right), \\
& \mathrm{E}\left(\mathrm{Y}_{1 \mathrm{i}} \mid \mathrm{x}_{\mathrm{i}}\right)=f_{1}\left(\mathrm{x}_{\mathrm{i}}^{\prime}\right)+\rho, \\
& x_{i}^{\prime}=x_{i}-x_{0},
\end{aligned}
$$

where $\mathrm{Y}_{0}$ and $\mathrm{Y}_{1}$ are the outcome variables before and after the policy, respectively. The running variable (year of birth or month-year of birth) is denoted by $\mathrm{x}$, which is normalized using the time of discontinuity $\left(\mathrm{x}_{0}\right)$. The relationships between the outcome variable and $\mathrm{x}$ before and after the discontinuity are denoted by $f_{0}($.$) and f_{1}($.$) , respectively. The policy effect on the outcome variables$ is denoted by $\rho$. Hence, we estimate the following model,

$$
Y_{i}=f_{0}\left(x_{i}^{\prime}\right)\left(1-D_{i}\right)+f_{1}\left(x_{i}^{\prime}\right) D_{i}+\rho D_{i}+\eta_{i},
$$

where $\mathrm{D}$ denotes the treatment variable, which is one when the running variable is above the threshold and zero otherwise. This threshold is January 1987. In equation (4), $\eta$ is the error term. While we 
take split time trends before and after the cut-off as in equation (4) in certain specifications, in others we take a single time trend by imposing $f_{0}()=.f_{1}()=.f($.$) . In addition, control variables for place$ and month of birth are added to equation (4) in robustness checks.

A key assumption of RDD is that the variation in the treatment variable is as good as randomized around the cut-off. This requires that individuals not manipulate their birth date to be on one particular side of the cut-off. In our context, since month-year of birth is determined well before the announcement of the policy, such a manipulation is not possible. Another critical assumption is that the relationship between the outcome variables and the running variable is continuous except at the cut-off. We check this assumption using placebo tests. First, we examine any potential effects of the policy on baseline covariates at the cut-off. The results, given in Table A3 of Appendix A, indicate no evidence of a policy effect on these covariates. Second, we search for discontinuities in the relationship between the outcome variable and the running variable away from the cut-off. The results of these checks, given in Tables A4 to A7 of Appendix A, do not indicate any obvious discontinuities.

A critical aspect of our identification strategy is to distinguish the discontinuous jump from the smooth function $\mathrm{f}($.$) or the split functions f_{0}(),. f_{1}($.$) . For this reason, we assess the robustness of our$ identification strategy to various choices. First, we take various time windows (bandwidths). Second, we take split polynomials on both sides of the cut-off, as well as a single polynomial throughout the time window. Third, we take very flexible polynomial speficiations to disentangle the time trends from the policy effect properly. The maximum degree of the polynomials taken depends on the width of time window and whether the running variable is month-year of birth or year of birth (both of which determine the number of data points on each side of the cut-off), as well as whether or not the time trends are different before and after the cut-off in a systematic way: the maximum order is higher when the time window is wider, when month-year of birth is the running variable, and when the time 
trends are assumed to be common on both sides of the cut-off. ${ }^{11}$ With sample (A), where the running variable is year of birth, the order of single and split polynomials goes up to four and two, respectively. When the running varable is month-year of birth, the order of single and split polynomials goes up to two and one, respectively, with sample (B1); up to four and two, respectively, with sample (B2); and up to six and three, respectively, with sample (B3). We take lower orders of polynomials when the time window is narrower because the risk of misspecification of the functional form is lower in that case. As argued by van der Klauuw (2008, p. 235) in his review of RDD, "A linear control function is likely to provide a reasonable approximation of the true functional form within a small neighborhood of the cut-off."12

Using month-year of birth as the running variable instead of year of birth imposes a weaker identification assumption. When we use year of birth as the running variable with sample (A), we compare birth cohorts that are three years apart around the cut-off. However, with month-year of birth as the running variable with samples (B1) to (B3), we compare birth cohorts that are 25 months apart around the cut-off. (In fact, we further narrow this to 19 months in the robustness checks of Section 6.4.) Another advantage of month-year of birth as the running variable is that it allows the specification of more flexible functional forms for the running variable (due to the higher number of data points on each side of the cut-off). On the other hand, the advantage of using sample (A) is the

\footnotetext{
${ }^{11}$ Cattaneo (2017) suggests using $4^{\text {th }}$-order or $5^{\text {th }}$-order polynomials in global polynomial approaches, but lower order polynomials for restricted bandwidths.

${ }^{12}$ We also use the Akaike information criterion to examine the relative performance of alternative specifications. Lowerorder polynomial specifications perform clearly better with 4-year intervals and generally better with 7-year intervals. At the same time, as we widen the time interval, the relative performance of high-order polynomials improves vis-à-vis the performance of low-order polynomials.
} 
higher number of observations, which becomes especially important at lower ages when the incidence of marriage and birth events is low.

If the timing of the education reform were correlated with some unobserved characteristics that also affect marriage and fertility decisions, we would get biased estimates. For instance, if the policy change had come right after some shock that decreased school enrollment rates while increasing marriage and fertility, there would have been a problem. In this sense, it is important to emphasize that the timing of the policy had to do with the political circumstances of the time.

Another potential concern is that the education reform coincides with other interventions that also influence the outcome of interest. This is especially the case if better-than-average economic conditions allow higher investment in public services on health, education, or social transfers. For this reason, we check for potential discontinuties in 1997 in a number of health (health expenditures per capita, infant and under-5 mortality rates), employment (employment rates of men and women aged 15 to 24), and growth outcomes (GDP per capita at ppp growth rate), as well as investment in education (education's share in public expenditure)—which are presented in Figure A2 of Appendix A. While there is a tremendous jump in the share of education in public expenditure, there are no visible discontinuities in the other outcomes. ${ }^{13}$ An exception to the absence of other interventions that could influence marriage and birth outcomes is the change in the Civil Code in 2002, whose effect we examine also using RDD.

In the estimation of the policy effect on marriage and birth outcomes by age, we run equation (4) for four outcome variables: i) ever-married status, ii) ever-given-birth status, iii) time to birth after

\footnotetext{
${ }^{13}$ Two other notable albeit substantially smaller scale educational interventions were implemented much later: the public conditional cash transfer (CCT) policy in 2003 and a NGO-driven CCT policy in 2005.
} 
marriage, and iv) number of children ever born. All equations are estimated at each age separately. We leave the inclusion of additional control variables as robustness checks because there is no variation in the outcome variable for some values of these variables in the regressions for lower ages. (In RDD, the inclusion of these variables would not matter for the estimated coefficients but it would matter for standard errors.) Standard errors are clustered at the level of birth-year or month-and-birthyear in accordance with the running variable.

\section{Results}

Before we present the estimation results from various samples and specifications outlined in the previous section, we would like to highlight the fact that the reason for our use of multiple samples is the trade-off between potential bias and precision. Sample (A) uses all available data from two different surveys and, therefore, offers more precision. However, in this global approach, the chances of misspecifying the functional form of the time trends are higher because the data cover a longer period. Hence, with sample (A), we experiment with various degrees of polynomials in defining the time trends and draw conclusions only if the results are robust across various specifications. As we zoom in around the cut-off gradually with samples (B3), (B2) and (B1) - as in local RDD applications - we decrease the chances of functional-form misspecification; however, this comes at the cost of lower statistical power. We refrain from using high-order polynomials as we zoom in around the cutoff - in particular with sample (B1) - as suggested by Gelman and Imbens (2014).

The use of sample (A) becomes critical at lower ages because, since the incidences of marriage and first-birth events are low at these ages, increasing the precision of the estimates becomes essential. On the other hand, as the running variable is year-of-birth with sample (A), the number of data points on each side of the cut-off is lower-which becomes even lower on the right-hand-side of the cut-off 
as age increases. Finally, while we show the results, separately, with split and with single polynomials for the time trends (for checking robustness), we prefer split polynomials as they are more flexible.

\subsection{Impact of the Education Policy on Schooling}

Table 1 presents estimation results on the policy effect on three schooling outcomes for women: $8^{\text {th }}$ grade completion in panel (A), $11^{\text {th }}$ grade (high school) completion in panel (B), and years of schooling in panel (C). The first two binary outcomes are estimated using logistic regressions, whereas years of schooling is estimated using OLS regression. Because of the minimum-age restrictions imposed (age 18 in panel (B) and age 22 in panel (C)), we are unable to use 10-year intervals around the donut-hole in panel (B) and 7-year and 10-year intervals around the donut-hole in panel $(\mathrm{C})$. (There are fewer data points on the right-hand side of the donut-hole than on the lefthand side in these cases.)

As can be seen in panel (A), there is strong evidence that the new policy increases the completion probability of grade 8 for women. Quantitatively, the policy increases the odds of the $8^{\text {th }}$-grade completion rate by a factor of about two. In addition, as suggested by Figure 1, the policy increases high school completion, which is not compulsory. Almost all coefficient estimates in panel (B) are statistically significant; and in the two cases where they are not, the coefficient magnitudes are very similar to those of the statistically significant coefficients. Panel (C) of Table 1 shows that the policy increases the years of schooling by about one year. ${ }^{14}$ All coefficients are statistically significant at

\footnotetext{
${ }^{14}$ When no donut-hole is taken, the estimated policy impact on years of schooling is about 0.75 years; i.e., the policy effect on years of schooling falls by more than 25 percent due to the fuzziness of treatment status right around the cutoff. This is the very reason we conduct our analysis with a donut-hole around the cut-off.
} 
least at the 5 percent level, except for one case where the magnitude of the coefficient estimate is very similar to the others.

\subsection{Policy Effect on Marriage and First-Birth of Teenage Women}

The estimation results, in terms of odds ratios, on the effects of the education policy on being ever married and on ever giving birth are presented for the full sample (sample A) in Table 2, where the running variable is year of birth, and for samples (B1) and (B2) in Table 3 and for sample (B3) in Table 4, where the running variable is month-year of birth. In these tables, the results for being ever married are given in panel (A)s, and the results for ever giving birth are given in panel (B)s. For samples (B1) to (B3), the minimum age for which results are presented is 13 for marriage and 15 for first-birth, whereas it is 12 for marriage and 13 for first-birth for sample (A) in Table 2 due to its larger sample size.

As can be seen in panel (A) of all Tables 2 to 4, for all time windows and with all specifications, there is evidence that the education policy decreases the probability of being ever married by ages 15 and 16-beyond the ages girls are mandated to stay in school with the new policy. To assess the magnitude of the effects, we prefer the estimates in Table 3, where we zoom in around the cut-off, because they are quite precise. The policy reduces the odds of being ever marrried by age 15 by a remarkable 60 to 70 percent and the odds of being ever married by age 16 by about 50 percent. Consistent with the rigid sequence of marriage and birth outcomes in Turkey, there is also evidence 
across all specifications in panel (B) of Tables 2-4 that the probabiliy of ever giving birth by ages 16 and 17 decreases with the policy ${ }^{15}$.

Tables 2-4 also show that the policy effect on being ever-married completely dissappears by age $17 .{ }^{16}$ Similarly, in the rigid sequence of marriage and fertility, the policy effect on ever giving birth disappears by age 18. In fact, the estimated odds ratios for marriage by age 17 and for birth by age 18 are close to one; thus, the lack of evidence of a policy effect in both cases is not due to high standard errors.

The results for sample (A) in Table 2 also show that the odds ratios for policy effect at ages 12 to 14 -when children are mandated to stay in school with the new policy - are substantially lower than one, indicating a negative effect of the policy on marriage. This effect is robust across all specifications at age 14, but not at lower ages presumably due to the low incidence of marriage events at these ages. The policy reduces the odds of ever being married by age 14 by at least 40 percent according to all specifications in Table 2. This indicates that the policy has a strong incarceration

\footnotetext{
${ }^{15}$ The results in Table 4, where the time window is wider, is more sensitive to the order of polynomials in time trends than those in Table 3, as expected. At the same time, the coefficients of the specifications with higher-order polynomials in Table 4 are more consistent with the coefficients in Table 3.

${ }^{16}$ While there is some evidence for an effect even after age 17 in column (1) of Table 2, this effect disappears with the more flexible specifications taken in other columns; and, there is no such evidence in Tables 3 and 4 where we zoom in around the cut-off.
} 
effect on marriage. Again, in accordance with the rigid sequence, there is evidence that the probability of ever giving birth by age 15 decreases with the policy. ${ }^{17}$

The results in Tables 3 and 4 also indicate that the estimated effects on marriage by ages 13 and 14 and on first-birth by age 15 are also substantially lower than one and of similar magnitude to the corresponding estimates in Table 2 . However, these estimates are generally statistically insignificant, unlike those in Table 2, due to the smaller sample sizes in these estimations.

Overall, the results are quite consistent across different time windows and running variables. At the same time, the estimates in Table 3-based on narrow time windows-are more stable across specifications than those in Table 2 and Table $4 .{ }^{18}$ Therefore, in quantifying the policy effect, we refer to the estimates in Table 3. Based on these estimates, we calculate the baseline and policy values of the predicted probabilities of being ever married and ever giving birth by age for the cohort born between December 1986 and January 1987. We present these in Table 5 for the specifications with split linear time trends. The magnitude of the policy effect on the probability of being ever married at ages 15 and 16 is striking; using sample (B2), the probability of marriage by age 15 decreases by 3.8 percentage points, from 6.5 to 2.7 percent, and that by age 16 drops by 5.7 percentage points, from 11.9 to 6.2 percent. In terms of percentage changes, the fall in the probability of marriage by age 15 is 58.5 percent, and that by age 16 is 47.9 percent. Similarly, the policy effect on first-birth

\footnotetext{
${ }^{17} \mathrm{We}$ also reduce sample (A) to 10-year intervals and 5-year intervals around the donut-hole and repeat our estimations with these samples. The results, presented in Tables A8 and A9 of Appendix A, are very consistent with those in Table 2.

${ }^{18}$ Finally, the estimates for both marriage and birth outcomes at higher ages are more stable across specifications in Tables 3 and 4 than in Table 2 because the data points on the right-hand side of the cut-off are even fewer at these higher age levels with sample (A).
} 
outcomes is also remarkable. According to the analysis with sample (B2), the probability of giving birth by age 16 decreases by 2.1 percentage points, from 3.4 to 1.3 percent, and the probability of giving birth by age 17 decreases by 4.6 percentage points, from 8.1 to 3.5 percent.

\subsubsection{Impact of the Education Policy on the Time to First-Birth after Marriage}

The drop in the percentage of women who give birth at young ages can be attributed to two different mechanisms. First, it could be brought about by a change in the age at marriage and, in fact, we illustrate above that the percentage of women who get married at young ages decreases with the policy. Second, as a result of the higher education levels caused by the education policy, married women could be delaying the birth of their first child. In this subsection, we tackle this question: after a woman is married, does the longer compulsory schooling increase the time to first-birth?

In this analysis, the sample includes only ever-married women, and the dependent variable is time to first-birth after marriage in months. The sample here is restricted to women who are 17 or older because, as shown in the previous section, the policy changes the group of ever-married women before age $17 .{ }^{19}$ Table 6 presents the OLS estimation results in two different panels. In panel (A), age at marriage is also controlled for, as this becomes a state variable here; in panel (B), the results without controlling for age at marriage (due to concerns about the endogeneity of this variable) are presented. Within each panel, the results for our four samples are presented separately. The estimates are quite similar across the two panels. The data present no evidence of a policy effect on the time to first-birth after marriage with any sample. This finding is important because, unlike the time to marriage and

\footnotetext{
${ }^{19}$ Figure A3 of the Appendix presents the time to first birth after marriage in number of months across the birth cohorts in our sample. This figure does not indicate any discontinuity.
} 
time to first-birth analyses, in the time to first-birth after marriage analysis we know for certain that women are out of school in the rigid sequence of schooling, marriage, and fertility in Turkey.

\subsubsection{Policy Effect on Number of Children Ever Born}

Here, we examine the policy effect on the number of children ever born to a woman. Using the information on the date of birth of all children, we construct histories of the number of children ever born to a woman in person-age format from age 15 to her current age in the sample, where age is in years and months. Then, we estimate the policy effect on the number of children ever born at selected values of women's age — at 12-month increments from 180 to 240 months; i.e., from age 15 to 20.

The policy effect on the number of children ever born is presented in Table 7 by women's age for samples (B1) and (B2). (Table A10 in Appendix A gives it for sample (B3).) For all time windows and specifications, there is strong evidence that the policy decreases the number of children born to a woman by age 18 . According to the estimates in Table 7 for 4 -year and 7 -year intervals with higherorder polynomials, the policy decreases the number of children born to an 18-year-old woman by about 0.07 to 0.08 . However, this effect is very short-lived; unlike what Dincer et al. (2014) and Gunes (2015) claim, there is no evidence of a policy effect on the number of children born to a 20year-old woman. This lack of significance is not due to large standard errors; the coefficient estimates are very close to zero-especially in comparison to the estimates for age 18 .

\subsection{Survival Analysis: Policy Effect on the Time to Marriage and Time to First-Birth}

The analysis in Section 6.2 uncovers the policy effect on marriage and first-birth outcomes by agewhich cumulates the age-specific effects over age. For instance, the policy effect on marriage by age 16 depends on the policy effect on the probability of marriage at each age conditional on not being married until that age (marriage hazard rate) by age 16. Even if the policy has no effect on the 
marriage hazard rate at age 16 , it could have an effect on marriage by age 16. Using survival analysis, we also estimate the policy effect on the timing of marriage and first-birth, thereby uncovering agespecific policy effects. The details of the analysis are given in Appendix B. The results show that while the marriage hazard rate at ages 15 and below decreases with the policy, once the students are out of school at age 17, they are more likely to get married as a result of the policy. Consequently, after age 17, the probability of being ever married reverts to its baseline levels as shown in Tables 24. Consistent with the rigid sequence of marriage and birth events, while the policy decreases the first-birth hazard rate at ages 15 to 17 , it increases it after age 18. As a result, the probability of ever giving birth by age 18 reverts to its pre-policy level.

\subsection{Robustness Checks}

Here, we briefly discuss the further robustness checks that we conduct; a detailed discussion is provided in Appendix C. First, we conduct falsification checks in which we examine the effects of policies that did not happen. For this purpose, we define counterfactual policy dummies by sliding the timing of the policy over time and estimate equation (4) using these. The results show that as we slide the timing of the policy away from its actual point, the policy effect gradually diminishes and vanishes - as would be expected. Second, we show that our findings are robust to the inclusion of other control variables. Third, we assess the sensitivity of our findings to the donut-hole width. We find that our findings are very robust when somewhat shorter or longer donut-holes (ranging from 9 to 18 months on each side of the donut-hole, instead of the baseline 12 months) are taken; nonetheless, with donut-holes that are narrower than 9 months on each side, fuzziness in the treatment status starts

to contaminate the estimates. Fourth, we examine the potential effects of the change in Civil Code in 2002 and find that there is either no or a small effect of the Civil Code on the marriage hazard rateespecially when compared to the magnitude of effect of the education policy. 


\section{Discussion}

In the conceptual framework discussion in Section 4, we identified two main channels through which education affects marriage and, therefore, birth outcomes: the incarceration effect and the human capital effect. Here we interpret our key findings within this framework.

We find a strong incarceration effect of the education policy on teenage marriage and births. The probability of being ever married by age 14 (girls are mandated to stay in school with the new education policy until this age) drops substantially, as shown in Table 2. At the same time, our findings show that the effect of the new education policy on teenage marriage and births persists for a few years beyond the new compulsory schooling years. While most children complete grade 8 by age 14 , we find that the effect of the policy on marriage persists by age 16 .

If there was only an incarceration effect of the policy on marriage, the women who delay their marriage because of the policy, which would otherwise happen at grade levels 6 to 8 (ages 12 to 14), would get married once the new compulsory schooling years are over. This 'catching-up effect' would mean that the hazard rate of marriage at ages 15 and 16 would be higher with the policy and, thus, the probability of marriage by age 15 or 16 would revert to its original level. However, this is not observed; the policy effect on the probability of marriage persists by age 16 (Tables 2-4). Moreover, it is not only the absence of a catching-up effect at ages 15 and 16 that results in the lower levels of ever-married status at these ages with the policy; in fact, as the survival analysis shows, the policy also reduces the hazard rate of marriage at age 15 (conditional on not being married until this 
age) - at an age that girls are no longer mandated to stay in school. These results, hence, indicate that there is also a human capital effect. ${ }^{20}$

However, our analysis also reveals that the marriage hazard rate at age 17 is higher with the policy than it would be in the absence of it; and, consequently, the probability of marriage reverts to its original level by this age. Therefore, the human capital effect of the policy on marriage is short-lived. In addition, we find no evidence of a human capital effect on the time to first-birth after marriage. An increase in the time to first-birth after marriage could not be a result of the incarceration effect of the policy because in the rigid sequence of schooling, marriage, and fertility in Turkey, all married women are out of school. In essence, we find a human capital effect of the policy on the time to marriage but no human capital effect on the time to first-birth after marriage.

Our estimated magnitude of the effect of the compulsory schooling policy on teenage fertility is much greater than those reported in developed countries. For instance, one of the largest effects Black et al. (2008) find is that the policy of mandating women to stay in school until age 17 reduces the probability of birth before age 19 by 8.8 percent in the US. In comparison, we find that mandating women in Turkey to stay in school until completing grade 8 (roughly age 14) reduces the probability of giving birth by age 17 by a striking 50 percent. In Western societies, teenage fertility may not necessarily be planned, whereas in Turkey, it is clearly planned along with marriage. Thus, schooling puts a very strong brake on teenage fertility in Turkey by preventing marriage, whereas it causes only a deceleration in the US and Norway by decreasing the chances of pregnancy. Nonetheless, similarly large effects are reported for other developing countries. For instance, Ozier (2015) reports that

\footnotetext{
${ }^{20}$ Black et al. (2008) find that the effect of compulsory schooling on teenage fertility in the US and in Norway also persists beyond the years the children are forced to stay in school and interpret this as evidence for human capital effect.
} 
secondary school completion in Kenya also causes a near elimination of pregnancy among compliers by age 18 .

Previous studies on early marriage in Turkey point out cultural factors, rather than economic factors, as the key driving elements. For instance, Edirne et al. (2010) find that the parents of teenage mothers have less education and are more likely to follow traditional matrimonies, but do not have lower household income than other parents. In their qualitative study in eastern Turkey, Ertem and Koçtürk (2008) highlight the importance of "protecting family honor" as a reason for marriage once girls reach

the age of menarche. If, in fact, cultural traditions are the main driving factor of early marriage and fertility, this study shows that it is not impossible to alter the course of these cultural traditions, at least in Turkey.

\section{Conclusion}

In this paper, we estimate the impact of the extension of compulsory schooling from 5 years to 8 in Turkey on the marriage and first-birth decisions of teenage women. We find that the rise in years of compulsory schooling indeed reduces the probability of marriage and giving birth for teenage women. Moreover, the magnitude of this effect is quite substantial. The proportion of women married by age 16 drops by 5.7 percentage points (48 percent), and the proportion of women who give birth by age 17 drops by 4.6 percentage points ( 57 percent) for the first birth-cohort affected by the policy. Nonetheless, shortly after these women are out of school, marriage and fertility hazard rates rebound. In fact, the marriage hazard rate at age 17 and the resulting fertility hazard rate at age 18 are higher with the policy than they would have been in the absence of it. Consequently, the policy effect on the probability of marriage by age 17 and the probability of first-birth by age 18 dissappear. In addition, we find no evidence for a policy effect on the time to first-birth after a woman is married. Hence, we conclude that there is a strong incarceration effect of the new compulsory schooling policy on 
marriage and, therefore, on first-birth in Turkey. While there is also evidence of a human capital effect on the time to marriage, the human capital effect on the probability of being ever-married vanish after a couple of years the students become free to leave school.

This study shows that increasing education by an extension of compulsory schooling years delays marriage and childbearing of teenage women in an upper-middle income (World Bank classification) developing country. Previous empirical literature shows that increasing the age at marriage and childbearing has important socioeconomic implications. Given the causal links between age at marriage and age at first-birth with other demographic outcomes (like divorce, the number and spacing of children), health outcomes (such as maternal and child mortality), and economic outcomes (labor force participation, migration, welfare take-up), as well as the inter-generational effects of early marriage and childbearing, the rise in age at marriage and age at first-birth would have important implications on the aggregate welfare. 


\section{References}

Alam N. Teenage Motherhood and Infant Mortality in Bangladesh. Journal of Biosocial Science 2000,$32 ; 229-236$.

Almond D., Doyle Jr. J., Kowalski A., Williams H. Estimating Marginal Returns to Medical Care: Evidence from At-risk Newborns. Quarterly Journal of Economics 2010, 125; 591-634.

Almond D., Doyle J.J. After Midnight: A Regression Discontinuity Design in Length of Postpartum Hospital Stays. American Economic Journal: Economic Policy 2011, 3; 1-34.

Axinn W.G., Barber J.S. Mass Education and Fertility Transition. American Sociological Review 2001, 66; 481-505.

Bajari P., Hong H., Park M. and R. Town. Regression Discontinuity Designs with an Endogenous Forcing Variable and an Application to Contracting in Health. NBER Working Paper 17463, December 2011.

Barreca A.I., Guldi M., Lindo J. and G. Waddell. Saving Babies? Revisiting the Effect of Very Low Birth Weight Classification. Quarterly Journal of Economics 2011, 126; 2117-2123.

Becker G. A Theory of Marriage, Part I. Journal of Political Economy 1973; 81; 813-846.

Black S., Devereux P., and Salvanes, K. Staying in the Classroom and Out of the Maternity Ward? The Effect of Compulsory Schooling Laws on Teenage Births. Economic Journal 2008; 118; 1025-1054.

Blossfeld H.P., and De Rose A. Educational Expansion and the Changes in Entry Into Marriage and Motherhood: The Experience of Italian Women. Genus 1992; 48(3-4); 73-89.

Brien M, Lillard L. Education, Marriage, and First Conception in Malaysia. Journal of Human Resources 1994; 29(4); 1167-1204. 
Card, D. and L. Giuliano. Does Gifted Education Work? For Which Students? NBER Working Paper No. 20453, September 2014.

Cattaneo M, Idrobo N., Titiunik R. A Practical Introduction to Regression Discontinuity Designs. Monograph prepared for Cambridge Elements: Quantitative and Computational Methods for Social Science. Cambridge University Press. May 29, 2017.

DeCicca, P., and Krashinsky, H. Does Education Reduce Teen Fertility? Evidence from Compulsory Schooling Laws (No. w21594). National Bureau of Economic Research (NBER); 2015.

Dincer, M.A., Kausal, N. and Grossman, M. “Women’s Education: Harbinger of Another Spring? Evidence form a Natural Experiment in Turkey”. World Development; 2014; 64; 243-258.

Edirne T., Can M., Kolusarı A., Yıldızhan R., Adalı E., Akdağ B. Trends, Characteristics, and Outcomes of Adolescent Pregnancy in Eastern Turkey. International Journal of Gynecology \& Obstetrics 2010, 110; 105-108

Field E., Ambrus A. Early marriage, age of menarche, and female schooling attainment in Bangladesh. Journal of Political Economy 2008; 116; 881-930.

Fukushima K., Mizuoka S., Yamamoto S., Iizuka T. Patient Cost Sharing and Medical Expenditures for the Elderly. Journal of Health Economics 2016, 45; 115-130.

Gelman, Andrew, and Guido Imbens. "Why high-order polynomials should not be used in regression discontinuity designs.” No. w20405. National Bureau of Economic Research, 2014.

Glewwe P. Why Does Mother's Schooling Raise Child Health in Developing Countries? Evidence from Morocco. Journal of Human Resources 1999; 34; 124-159.

Glewwe P. Schools and Skills in Developing Countries: Education Policies and Socioeconomic Outcomes. Journal of Economic Literature 2002; 40(2); 436-482. 
Grossman M. On the concept of health capital and the demand for health. Journal of Political Economy $1972 ; 80 ; 223-255$.

Grönqvist, H., and Hall, C. "Education Policy and Early Fertility: Lessons from an Expansion of Upper Secondary Schooling”. Economics of Education Review; 2013; 37; 13-33.

Gulesci, S. and Meyersson, E. "'For the Love of the Republic' Education, Secularism, and Empowerment.” Working Paper 490. IGIER, Bocconi University; 2013.

Gunes,, P.M. "The Impact of Female Education on Fertility: Evidence from Turkey." Grand Challenges Canada Working Paper GCC 13-01; 2013

Hahn J., Todd P.E., van der Klauuw V. Identification and Estimation of Treatment Effects with a Regression-Discontinuity Design. Econometrica 2001; 69; 201-9.

Hoxby C. and G.B. Bulman. The Effects of the Tax Deduction for Postsecondary Tuition: Implications for Structuring Tax-based Aid. Economics of Education Review 2016, 51; 23 60.

International Center for Research on Women. Child Marriage Facts and Figures. http://www.icrw.org/child-marriage-facts-and-figures, accessed on October 4, 2015.

Kırdar M., Dayığlu M. and Koc I. Does Longer Compulsory Education Equalize Schooling by Gender and Rural/Urban Residence? World Bank Economic Review 2016, 30(3): 549-579.

Kırdar M., Dayığlu M. and Koc I. The Impact of Schooling on the Timing of Marriage and Fertility: Evidence from a Change in Compulsory Schooling Law. MPRA Working Paper No: 13410 from University Library of Munich, Germany, February 2009.

Lee D.S. "Randomized Experiments from Non-random Selection in U.S. House Elections," Journal of Econometrics 2008; 142(2); 675-697. 
Lee D.S., Lemieux T. Regression Discontinuity Designs in Economics. Journal of Economic Literature 2010, 48(2); 281-355.

Lefgren L., McIntyre F. The Relationship between Women's Education and Marriage Outcomes. Journal of Labor Economics 2006; 24(4); 787-830.

Lloyd C.B., Mensch B.S. Marriage and Childbirth as Factors in Dropping out from School: an Analysis of DHS Data from Sub-Saharan Africa. Population Studies 2008; 62; 1-13.

Mason K.O. The Status of Women: Conceptual and Methodological Debates in Demographic Studies. Sociological Forum 1986; 1; 284-300

Marini M. Women's Educational Attainment and the Timing of Entry into Parenthood. American Sociological Review 1984; 49; 491-511.

McCrary J., Royer H. The effect of female education on fertility and infant health: evidence from school entry policies using exact date of birth. American Economic Review $2011 ; 101 ; 158-$ 195.

Ozier O. The impact of secondary schooling in Kenya: a regression-discontinuity analysis. The World Bank, Policy Research Working Paper 7384; 2015.

Raj A., Saggurti N., Balaiah D., Silverman J.G. Prevalence of Child Marriage and its Effect on Fertility and Fertility-control Outcomes of Young Women in India: a Cross-Sectional, Observational Study. Lancet 2009; 373; 1883-89.

Raj A., Saggurti N., Winter M., Labonte A., Decker M.R., Balaiah D., Silverman J.G. The Effect of Maternal Child Marriage on Morbidity and Mortality of Children under 5 in India. British Medical Journal 2010; 340; Article Number: b4258. 
Riddell, A. "The Introduction of Free Primary Education in sub-Saharan Africa," Background paper prepared for the Education for All Global Monitoring Report 2003/4, The Leap to Equality.

Silles, M.A. "The Effect of Schooling on Teenage Childbearing: Evidence Using Changes in Compulsory Education Laws” Journal of Population Economics 2011; 24: 761-777.

Skirbekk V, Kohler HP, Prskawetz A. Birth Month, School Graduation, and the Timing of Births and Marriages. Demography 2004; 41(3); 547-568.

Thornton, A., Axinn W.G., Teachman J.D. The Influence of School Enrollment and Accumulation on Cohabitation and Marriage in Early Adulthood. American Sociological Review 1995; 60; 762-774.

Turkish Ministry of Education. National Education Statistics: Formal Education. Ankara, 2011.

Turkish Statistical Institute. Population and Development Indicators. http://nkg.tuik.gov.tr/goster.asp?aile=3.UNICEF, UNICEF Data: Monitoring the Situation of Children and Women, https://data.unicef.org/topic/child-protection/child-marriage/, accessed on April 10, 2017

van der Klauuw, W. (2008) "Regression-Discontinuity Analysis: A Survey of Recent Developments in Economics," Labour: Review of Labour Economics and Industrial Relations 22(2): 219245.

Willis K.J. A New Approach to the Economic Theory of Fertility Behavior. Journal of Political Economy 1973; 81(2); S14-64. 
Figure 1: Policy Effect on Schooling Outcomes

A) Running Variable: Year of Birth

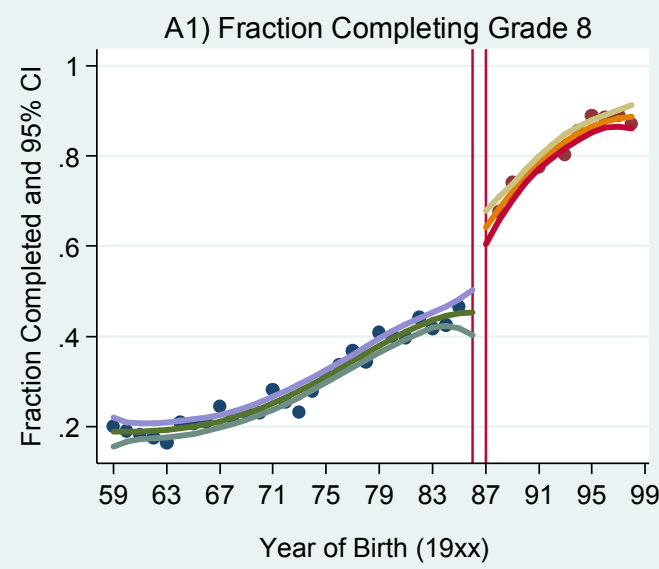

A2) Fraction Completing Grade 11

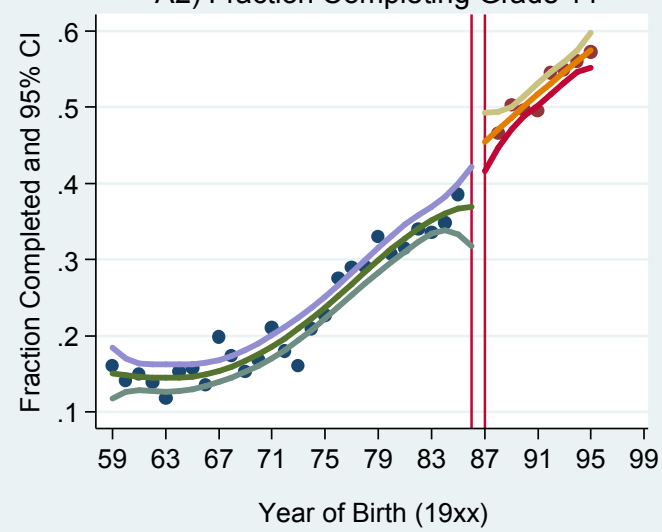

A3) Average Years of Schooling

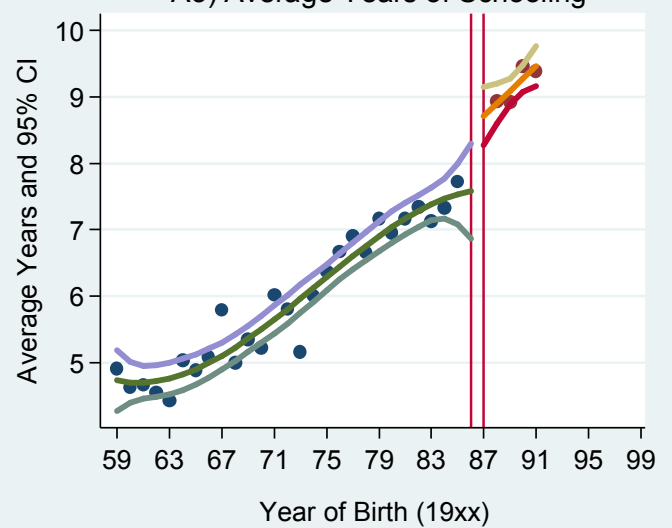

B) Running Variable: Month-Year of Birth

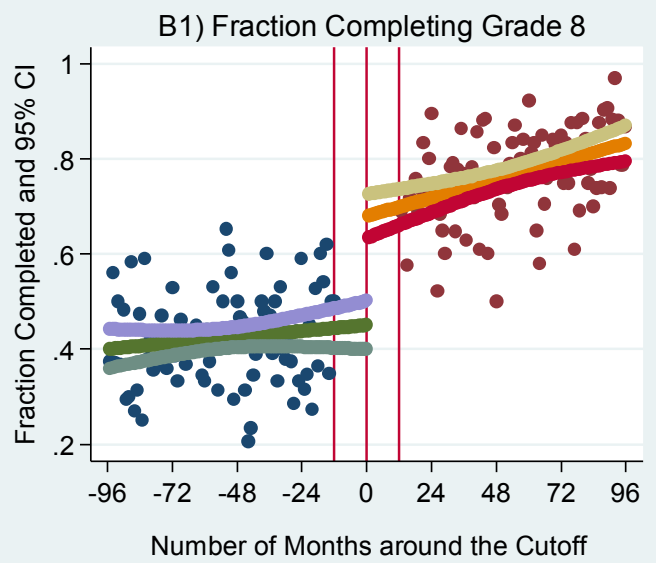

B2) Fraction Completing Grade 11

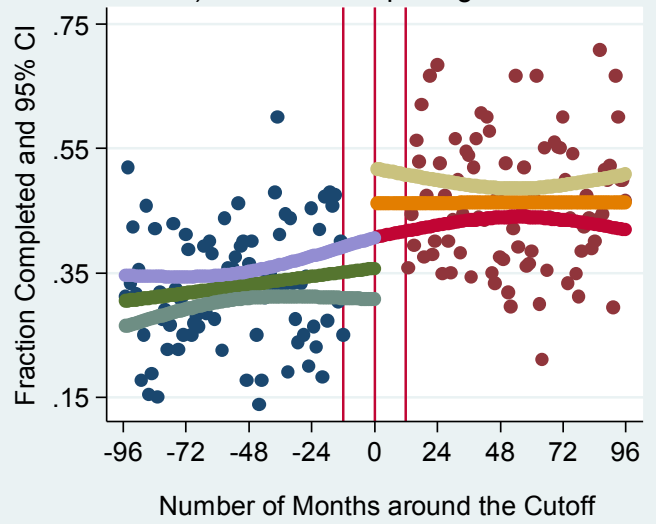

B3) Average Years of Schooling

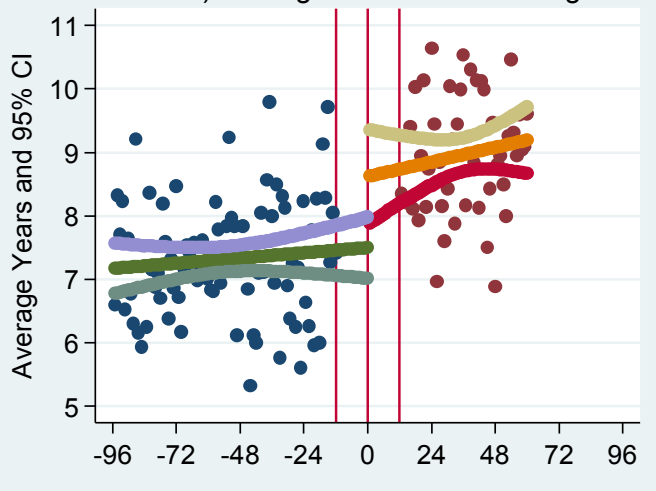

Number of Months around the Cutoff

Notes: a) The sample is drawn from both the 2008 and 2013 DHS in panel (A), but only from the 2013 DHS in panel (B).

b) In both panels, 1986 and 1987 birth cohorts are excluded due to the fuzziness of treatment status

d) Quartic polynomials are fit before the cut-off in panel (A), whereas after the cut-off quadratic polynomials are fit in panels (A1) and (A2), but linear in panel (A3).

e) Linear polynomials are fit before and after the cut-off in panel (B),
f) In panels (A2), (A3), and (B3), the graphs are truncated on the right hand side due to higher age restrictions. 
Figure 2: Fractions of Women Ever Married at Selected Ages by Year of Birth
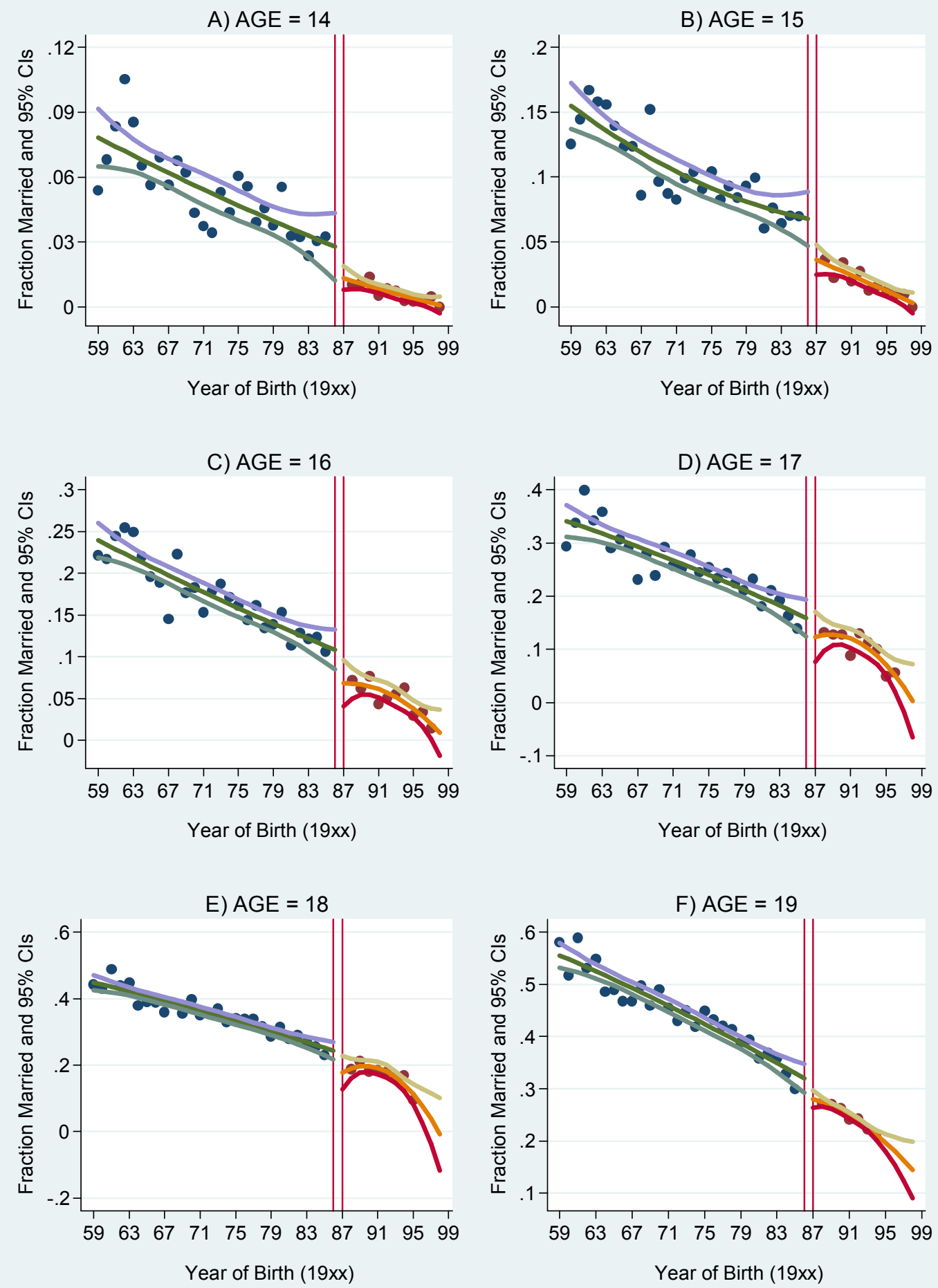

Notes: a) The sample is drawn from both the 2008 and 2013 DHS

b) In both panels, 1986 and 1987 birth cohorts are excluded due to the fuzziness of treatment status. c) Quartic polynomials are fit before the cut-off, quadratic polynomials are fit after the cut-off. 
Figure 3: Fraction of Women Ever Married at Selected Ages by Month-Year of Birth
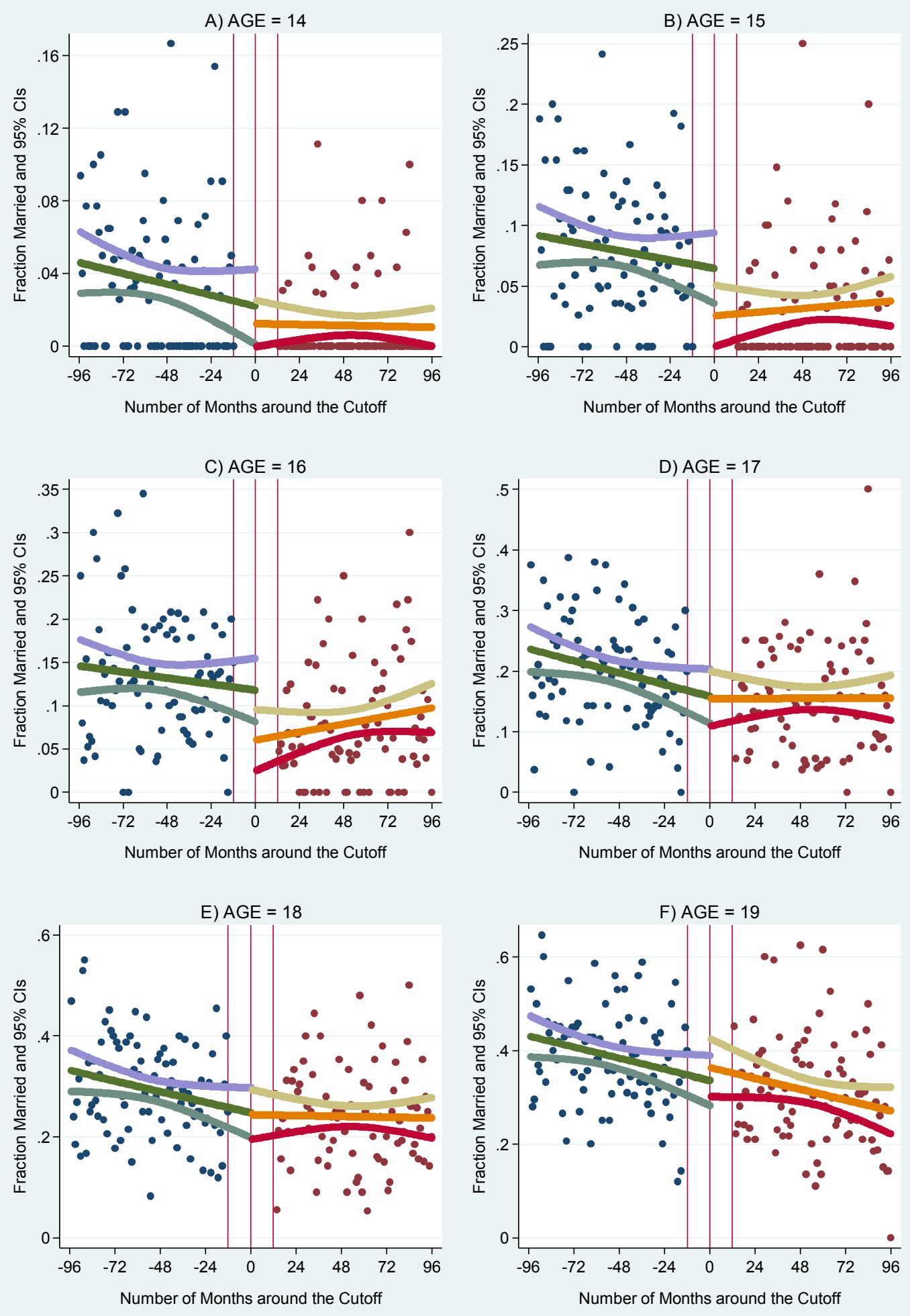
Figure 4: Fractions of Women Ever Given Birth at Selected Ages by Year of Birth
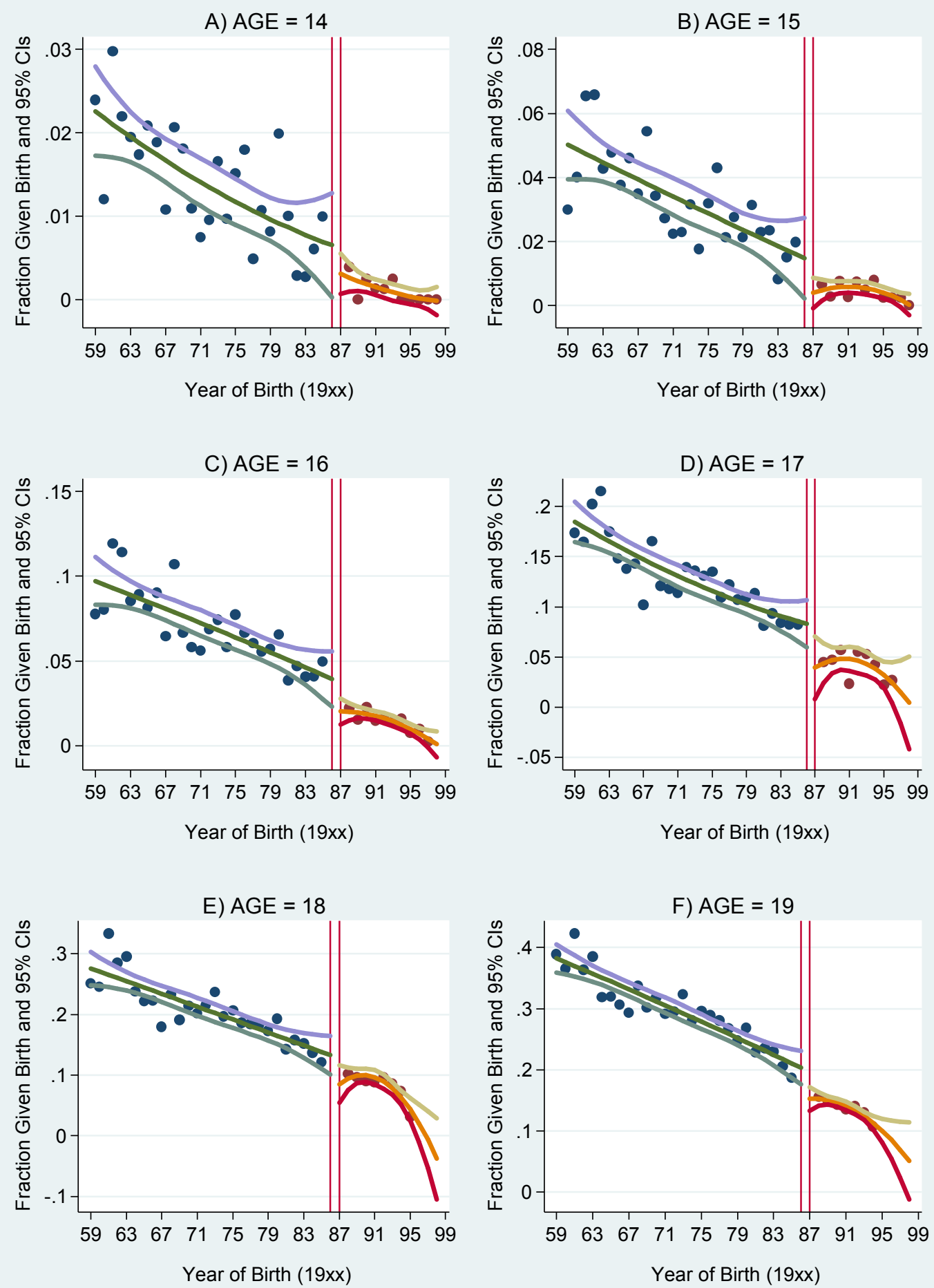

Notes: a) The sample is drawn from both the 2008 and 2013 DHS.

b) In both panels, 1986 and 1987 birth cohorts are excluded due to the fuzziness of treatment status. c) Quartic polynomials are fit before the cut-off, quadratic polynomials are fit after the cut-off. 
Figure 5: Fraction of Women Ever Given Birth at Selected Ages by Month-Year of Birth
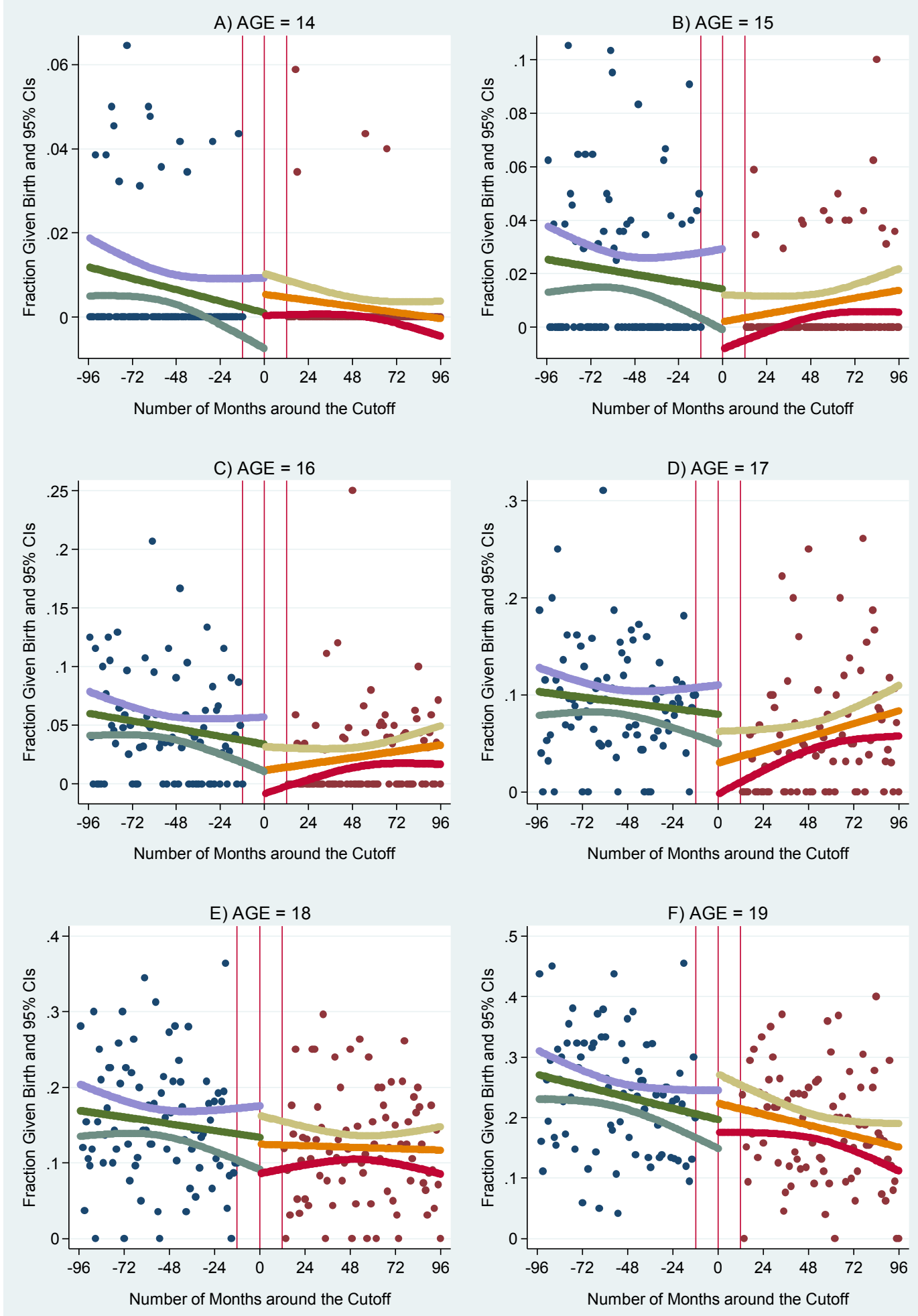


\section{Table 1: Policy Effect on Schooling Outcomes, Odds ratios}

\begin{tabular}{|c|c|c|c|c|c|c|c|c|c|c|}
\hline \multirow[b]{2}{*}{ Degree of Polynomial } & \multicolumn{6}{|c|}{ Single Time Trend } & \multicolumn{3}{|c|}{ Split Time Trends } & \\
\hline & $\begin{array}{l}\text { One } \\
\text { (1) }\end{array}$ & $\begin{array}{l}\text { Two } \\
\text { (2) }\end{array}$ & $\begin{array}{c}\text { Three } \\
\text { (3) }\end{array}$ & $\begin{array}{l}\text { Four } \\
\text { (4) }\end{array}$ & $\begin{array}{l}\text { Five } \\
\text { (5) }\end{array}$ & $\begin{array}{l}\text { Six } \\
\text { (6) }\end{array}$ & $\begin{array}{l}\text { One } \\
\text { (7) }\end{array}$ & $\begin{array}{l}\text { Two } \\
\text { (8) }\end{array}$ & $\begin{array}{l}\text { Three } \\
\text { (9) }\end{array}$ & Obs. \\
\hline
\end{tabular}

A) Policy Effect on the Odds of Completing Grade 8 - Logistic Regression Results

\begin{tabular}{|c|c|c|c|c|c|c|c|c|c|c|}
\hline Year of Birth & $2.806 * * *$ & $1.906 * * *$ & $1.909 * * *$ & $2.120 * * *$ & -- & -- & $2.033 * * *$ & $1.683 * * *$ & -- & 21,845 \\
\hline 1959-1998 & [0.269] & [0.203] & [0.189] & {$[0.272]$} & -- & -- & {$[0.140]$} & {$[0.196]$} & -- & \\
\hline Month-Year of Birth & $2.520 * * *$ & $2.331 * * *$ & -- & -- & -- & -- & $2.375 * * *$ & -- & -- & 2,325 \\
\hline 4-year intervals & [0.603] & {$[0.526]$} & -- & -- & -- & -- & {$[0.537]$} & -- & -- & \\
\hline Month-Year of Birth & $2.590 * * *$ & $2.420 * * *$ & $2.439 * * *$ & $2.461 * * *$ & -- & -- & $2.442 * * *$ & $2.361 * * *$ & -- & 4,065 \\
\hline 7-year intervals & {$[0.447]$} & [0.397] & {$[0.613]$} & [0.622] & -- & -- & [0.399] & {$[0.740]$} & -- & \\
\hline Month-Year of Birth & $2.114 * * *$ & $1.906 * * *$ & $2.702 * * *$ & $2.589 * * *$ & $2.657 * * *$ & $2.661 * * *$ & $1.921 * * *$ & $2.894 * * *$ & $2.284 *$ & 5,945 \\
\hline 10 -year intervals & [0.296] & {$[0.252]$} & {$[0.531]$} & {$[0.514]$} & {$[0.708]$} & {$[0.728]$} & {$[0.250]$} & {$[0.683]$} & {$[0.967]$} & \\
\hline
\end{tabular}

B) Policy Effect on the Odds of Completing Grade 11 - Logistic Regression Results

\begin{tabular}{|c|c|c|c|c|c|c|c|c|c|c|}
\hline Year of Birth & $1.257 * * *$ & 1.131 & $1.167 * * *$ & $1.296 * * *$ & -- & -- & $1.274 * * *$ & $1.180 * *$ & -- & 19,321 \\
\hline 1959-1995 & {$[0.062]$} & {$[0.085]$} & {$[0.066]$} & {$[0.110]$} & -- & -- & {$[0.056]$} & {$[0.085]$} & -- & \\
\hline Month-Year of Birth & $1.799 * *$ & $1.791 * *$ & -- & -- & -- & -- & $1.784 * *$ & -- & -- & 2,325 \\
\hline 4-year intervals & {$[0.472]$} & {$[0.464]$} & -- & -- & -- & -- & {$[0.462]$} & -- & -- & \\
\hline Month-Year of Birth & $1.500 * *$ & $1.497 * *$ & $1.691 *$ & $1.665^{*}$ & -- & -- & $1.493 * *$ & 1.696 & -- & 4,065 \\
\hline 7-year intervals & {$[0.256]$} & {$[0.255]$} & {$[0.481]$} & {$[0.462]$} & -- & -- & {$[0.254]$} & {$[0.601]$} & -- & \\
\hline \multicolumn{11}{|c|}{ C) Policy Effect on Years of Schooling - OLS Regression Results } \\
\hline Year of Birth & $1.039 * * *$ & $0.708 * * *$ & $1.240 * * *$ & $1.030 * * *$ & -- & -- & $0.858 * * *$ & 0.915 & -- & 16,448 \\
\hline 1959-1991 & {$[0.135]$} & {$[0.248]$} & {$[0.350]$} & {$[0.264]$} & -- & -- & {$[0.195]$} & {$[0.560]$} & -- & \\
\hline Month-Year of Birth & $1.043^{*}$ & $1.024 *$ & -- & -- & -- & -- & $1.034 *$ & -- & -- & 2,293 \\
\hline 4-year intervals & {$[0.530]$} & {$[0.528]$} & -- & -- & -- & -- & {$[0.528]$} & -- & -- & \\
\hline
\end{tabular}

Notes: The sample includes observations from both the 2008 and 2013 DHS in the analysis by year of birth, but only from the 2013 DHS in the analysis by month-year of birth. The sample is restricted to ages 15 and above in panel (A), to ages 18 and above in panel (B), and to ages 22 and above in panel (C). In panel (B), 10-year intervals on both sides of the cut-off cannot be taken due to the age restriction. Due to the same reason, in panel (C), neither 7-year intervals nor 10-year intervals can be taken. In all analyses, 1986 and 1987 birth cohorts are excluded. The policy dummy is one when year of birth is greater 1987. Each cell comes from a separate regresion of the specified schooling outcome on the policy dummy as well as the specified time trends. In panels (A) and (B), odds ratios and their standard errors are given, wheras in panel (C), OLS estimates are given. Standard errors are clustered at the year-of-birth level when the running variable is year of birth and at the month-year of birth level when the running variable is month-year of birth. Statistical significance is *** at 1 percent level, ** at 5 percent level, * at 10 percent level. 
Table 2: Policy Effect on Marriage and First-Birth by Age, Odds Ratios - Full Sample (Sample A) and Year of Birth as the Running Variable

\begin{tabular}{|c|c|c|c|c|c|c|c|}
\hline & \multicolumn{4}{|c|}{ Single Time Trend } & \multicolumn{2}{|c|}{ Split Time Trends } & \multirow[b]{2}{*}{ Obs. } \\
\hline & $\begin{array}{l}\text { Linear } \\
(1)\end{array}$ & $\begin{array}{c}\text { Quadratic } \\
\text { (2) }\end{array}$ & $\begin{array}{c}\text { Cubic } \\
\text { (3) }\end{array}$ & $\begin{array}{c}\text { Quartic } \\
\text { (4) }\end{array}$ & $\begin{array}{c}\text { Linear } \\
(5)\end{array}$ & $\begin{array}{c}\text { Quadratic } \\
\text { (6) }\end{array}$ & \\
\hline \multicolumn{8}{|c|}{ A) Dependent Variable: Ever Married } \\
\hline Age $=12$ & $\begin{array}{c}0.249 * * * \\
{[0.118]}\end{array}$ & $\begin{array}{c}0.597 \\
{[0.366]}\end{array}$ & $\begin{array}{c}0.488 \\
{[0.309]}\end{array}$ & $\begin{array}{c}0.415 \\
{[0.288]}\end{array}$ & $\begin{array}{c}0.333^{*} \\
{[0.199]}\end{array}$ & $\begin{array}{c}0.690 \\
{[0.492]}\end{array}$ & 20,552 \\
\hline Age $=13$ & $\begin{array}{c}0.329 * * * \\
{[0.083]}\end{array}$ & $\begin{array}{c}0.590 \\
{[0.247]}\end{array}$ & $\begin{array}{c}0.604 \\
{[0.241]}\end{array}$ & $\begin{array}{c}0.487^{*} \\
{[0.213]}\end{array}$ & $\begin{array}{c}0.572 * \\
{[0.183]}\end{array}$ & $\begin{array}{c}0.791 \\
{[0.356]}\end{array}$ & 20,552 \\
\hline Age $=14$ & $\begin{array}{c}0.305 * * * \\
{[0.056]}\end{array}$ & $\begin{array}{c}0.438 * * * \\
{[0.108]}\end{array}$ & $\begin{array}{c}0.601 * * \\
{[0.137]}\end{array}$ & $\begin{array}{c}0.546 * * \\
{[0.130]}\end{array}$ & $\begin{array}{c}0.572 * * \\
{[0.129]}\end{array}$ & $\begin{array}{c}0.387 * * * \\
{[0.108]}\end{array}$ & 20,552 \\
\hline Age $=15$ & $\begin{array}{c}0.389 * * * \\
{[0.061]}\end{array}$ & $\begin{array}{c}0.528 * * * \\
{[0.120]}\end{array}$ & $\begin{array}{c}0.701 * * \\
{[0.123]}\end{array}$ & $\begin{array}{c}0.611 * * * \\
{[0.098]}\end{array}$ & $\begin{array}{c}0.729^{*} \\
{[0.120]}\end{array}$ & $\begin{array}{l}0.494 * * \\
{[0.148]}\end{array}$ & 20,552 \\
\hline Age $=16$ & $\begin{array}{c}0.556 * * * \\
{[0.056]}\end{array}$ & $\begin{array}{c}0.744 * * \\
{[0.098]}\end{array}$ & $\begin{array}{c}0.828 \\
{[0.102]}\end{array}$ & $\begin{array}{c}0.777 * * \\
{[0.086]}\end{array}$ & $\begin{array}{c}0.785^{*} \\
{[0.098]}\end{array}$ & $\begin{array}{c}0.610^{* * *} \\
{[0.105]}\end{array}$ & 19,732 \\
\hline Age $=17$ & $\begin{array}{c}0.711 * * * \\
{[0.072]}\end{array}$ & $\begin{array}{c}0.979 \\
{[0.115]}\end{array}$ & $\begin{array}{c}1.073 \\
{[0.124]}\end{array}$ & $\begin{array}{c}1.127 \\
{[0.148]}\end{array}$ & $\begin{array}{c}0.932 \\
{[0.094]}\end{array}$ & $\begin{array}{c}0.775 \\
{[0.150]}\end{array}$ & 18,879 \\
\hline Age $=18$ & $\begin{array}{c}0.749 * * * \\
{[0.050]}\end{array}$ & $\begin{array}{c}0.922 \\
{[0.087]}\end{array}$ & $\begin{array}{c}0.985 \\
{[0.128]} \\
\end{array}$ & $\begin{array}{c}1.027 \\
{[0.120]}\end{array}$ & $\begin{array}{c}0.874 \\
{[0.109]} \\
\end{array}$ & $\begin{array}{c}0.655 * * * \\
{[0.072]}\end{array}$ & 18,043 \\
\hline \multicolumn{8}{|c|}{ B) Dependent Variable: Ever Given Birth } \\
\hline Age $=13$ & $\begin{array}{c}0.282 * * \\
{[0.158]}\end{array}$ & $\begin{array}{c}0.885 \\
{[0.736]}\end{array}$ & $\begin{array}{c}1.385 \\
{[1.129]}\end{array}$ & $\begin{array}{c}1.520 \\
{[1.196]}\end{array}$ & $\begin{array}{c}1.217 \\
{[0.598]}\end{array}$ & $\begin{array}{c}0.137 \\
{[0.229]}\end{array}$ & 20,552 \\
\hline Age $=14$ & $\begin{array}{c}0.255^{* * *} \\
{[0.105]}\end{array}$ & $\begin{array}{c}0.417 \\
{[0.267]}\end{array}$ & $\begin{array}{c}0.702 \\
{[0.455]}\end{array}$ & $\begin{array}{c}0.669 \\
{[0.428]}\end{array}$ & $\begin{array}{c}0.652 \\
{[0.338]}\end{array}$ & $\begin{array}{c}0.335 \\
{[0.374]}\end{array}$ & 20,552 \\
\hline Age $=15$ & $\begin{array}{c}0.353 * * * \\
{[0.077]}\end{array}$ & $\begin{array}{c}0.446 * * \\
{[0.153]}\end{array}$ & $\begin{array}{c}0.486 * * \\
{[0.160]}\end{array}$ & $\begin{array}{l}0.411 * * \\
{[0.153]}\end{array}$ & $\begin{array}{c}0.444 * * * \\
{[0.130]}\end{array}$ & $\begin{array}{l}0.209 * * \\
{[0.128]}\end{array}$ & 20,552 \\
\hline Age $=16$ & $\begin{array}{c}0.425 * * * \\
{[0.054]}\end{array}$ & $\begin{array}{c}0.587 * * * \\
{[0.118]}\end{array}$ & $\begin{array}{c}0.678 * * \\
{[0.129]}\end{array}$ & $\begin{array}{c}0.610 * * * \\
{[0.114]}\end{array}$ & $\begin{array}{c}0.639 * * * \\
{[0.104]}\end{array}$ & $\begin{array}{c}0.454 * * * \\
{[0.121]}\end{array}$ & 19,732 \\
\hline Age $=17$ & $\begin{array}{c}0.567 * * * \\
{[0.067]}\end{array}$ & $\begin{array}{c}0.651 * * * \\
{[0.091]}\end{array}$ & $\begin{array}{c}0.710 * * \\
{[0.107]}\end{array}$ & $\begin{array}{c}0.773 * \\
{[0.112]}\end{array}$ & $\begin{array}{c}0.638 * * * \\
{[0.093]}\end{array}$ & $\begin{array}{c}0.500 * * \\
{[0.142]}\end{array}$ & 18,873 \\
\hline Age $=18$ & $\begin{array}{c}0.677 * * * \\
{[0.056]} \\
\end{array}$ & $\begin{array}{c}0.873 \\
{[0.085]}\end{array}$ & $\begin{array}{c}1.003 \\
{[0.127]}\end{array}$ & $\begin{array}{c}1.047 \\
{[0.117]}\end{array}$ & $\begin{array}{c}0.850 \\
{[0.100]}\end{array}$ & $\begin{array}{l}0.600 * * \\
{[0.127]}\end{array}$ & 18,033 \\
\hline
\end{tabular}

Notes: The sample includes observations from both 2008 and 2013 DHS. In the 2008 survey, it includes 1959 to 1993 birth cohorts in all rows of age values above; in the 2013 survey, it includes 1964 to 1998 birth cohorts for ages 12 to 15,1964 to 1997 birth cohorts for age 16 , 1964 to 1996 birth cohorts for age 17, and 1964 to 1995 birth cohorts for age 18.1986 and 1987 birth cohorts are excluded in all cases. The policy dummy is one when year of birth is greater than 1987. Each cell comes from a separate logistic regression of ever-married status at the specified age in panel (A) and of ever-given-birth status at the specified age in panel (B) on the policy variable as well as the specified time trends. Odds ratios and their standard errors are given as estimated parameters. In the first 4 columns, single time trends up to quartic polynomials are fitted, whereas separate polynomials are fitted on either side of the discontinuity in columns (5) and (6). The standard errors are clustered at the year-of-birth level. Statistical significance is *** at 1 percent level, ** at 5 percent level, * at 10 percent level. 
Table 3: Policy Effect on Marriage and First-Birth by Age, Odds Ratios - Month-Year of Birth as Running Variable, 4-Year and 7-Year Intervals around the Donut-hole (Samples B1, B2)

\begin{tabular}{|c|c|c|c|c|c|c|c|c|c|}
\hline \multirow[b]{3}{*}{$\begin{array}{l}\text { Degree of } \\
\text { Polynomial } \\
\end{array}$} & \multicolumn{3}{|c|}{ I) 4-Year Intervals } & \multicolumn{6}{|c|}{ II) 7-Year Intervals } \\
\hline & \multicolumn{2}{|c|}{$\begin{array}{c}\text { Single Time } \\
\text { Trend }\end{array}$} & \multirow{2}{*}{$\begin{array}{c}\begin{array}{c}\text { Split } \\
\text { Trends }\end{array} \\
\text { One } \\
(3)\end{array}$} & \multicolumn{4}{|c|}{ Single Time Trend } & \multicolumn{2}{|c|}{ Split Time Trends } \\
\hline & $\begin{array}{l}\text { One } \\
(1)\end{array}$ & $\begin{array}{l}\text { Two } \\
(2) \\
\end{array}$ & & $\begin{array}{l}\text { One } \\
(4)\end{array}$ & $\begin{array}{l}\text { Two } \\
(5) \\
\end{array}$ & $\begin{array}{c}\text { Three } \\
(6)\end{array}$ & $\begin{array}{c}\text { Four } \\
(7)\end{array}$ & $\begin{array}{c}\text { One } \\
(8)\end{array}$ & $\begin{array}{l}\text { Two } \\
(9) \\
\end{array}$ \\
\hline \multicolumn{10}{|c|}{ A) Dependent Variable: Ever Married } \\
\hline Age $=13$ & $\begin{array}{c}0.451 \\
{[0.478]}\end{array}$ & $\begin{array}{c}0.412 \\
{[0.458]}\end{array}$ & $\begin{array}{c}0.385 \\
{[0.445]}\end{array}$ & $\begin{array}{c}0.883 \\
{[0.697]}\end{array}$ & $\begin{array}{c}1.066 \\
{[0.774]}\end{array}$ & $\begin{array}{c}0.812 \\
{[0.943]}\end{array}$ & $\begin{array}{c}0.293 \\
{[0.416]}\end{array}$ & $\begin{array}{c}0.971 \\
{[0.708]}\end{array}$ & $\begin{array}{c}0.194 \\
{[0.371]}\end{array}$ \\
\hline Age $=14$ & $\begin{array}{c}0.356 \\
{[0.299]}\end{array}$ & $\begin{array}{c}0.288 \\
{[0.263]}\end{array}$ & $\begin{array}{c}0.282 \\
{[0.259]}\end{array}$ & $\begin{array}{c}0.777 \\
{[0.451]}\end{array}$ & $\begin{array}{c}0.775 \\
{[0.457]}\end{array}$ & $\begin{array}{c}0.439 \\
{[0.381]}\end{array}$ & $\begin{array}{l}0.175^{*} \\
{[0.183]}\end{array}$ & $\begin{array}{c}0.742 \\
{[0.436]}\end{array}$ & $\begin{array}{c}0.113^{*} \\
{[0.148]}\end{array}$ \\
\hline Age $=15$ & $\begin{array}{c}0.321 * * \\
{[0.153]}\end{array}$ & $\begin{array}{c}0.305 * * \\
{[0.172]}\end{array}$ & $\begin{array}{c}0.285 * * \\
{[0.161]}\end{array}$ & $\begin{array}{c}0.449 * * \\
{[0.159]}\end{array}$ & $\begin{array}{c}0.406 * * \\
{[0.163]}\end{array}$ & $\begin{array}{c}0.310 * * \\
{[0.178]}\end{array}$ & $\begin{array}{c}0.232 * * \\
{[0.145]}\end{array}$ & $\begin{array}{c}0.395 * * \\
{[0.161]}\end{array}$ & $\begin{array}{l}0.159 * * \\
{[0.126]}\end{array}$ \\
\hline Age $=16$ & $\begin{array}{l}0.521 * * \\
{[0.167]}\end{array}$ & $\begin{array}{c}0.494 * * \\
{[0.174]}\end{array}$ & $\begin{array}{c}0.483 * * \\
{[0.167]}\end{array}$ & $\begin{array}{c}0.540 * * * \\
{[0.128]}\end{array}$ & $\begin{array}{c}0.500 * * * \\
{[0.125]}\end{array}$ & $\begin{array}{l}0.518^{*} \\
{[0.198]}\end{array}$ & $\begin{array}{c}0.442 * * \\
{[0.167]}\end{array}$ & $\begin{array}{c}0.492 * * * \\
{[0.124]}\end{array}$ & $\begin{array}{c}0.414^{*} \\
{[0.192]}\end{array}$ \\
\hline Age $=17$ & $\begin{array}{c}1.359 \\
{[0.406]}\end{array}$ & $\begin{array}{c}1.313 \\
{[0.397]}\end{array}$ & $\begin{array}{c}1.318 \\
{[0.397]}\end{array}$ & $\begin{array}{c}1.043 \\
{[0.204]}\end{array}$ & $\begin{array}{c}1.026 \\
{[0.199]}\end{array}$ & $\begin{array}{c}1.252 \\
{[0.422]}\end{array}$ & $\begin{array}{c}1.158 \\
{[0.390]}\end{array}$ & $\begin{array}{c}1.021 \\
{[0.198]}\end{array}$ & $\begin{array}{c}1.284 \\
{[0.571]}\end{array}$ \\
\hline Age $=18$ & $\begin{array}{c}1.128 \\
{[0.297]}\end{array}$ & $\begin{array}{c}1.119 \\
{[0.297]}\end{array}$ & $\begin{array}{c}1.120 \\
{[0.295]}\end{array}$ & $\begin{array}{c}1.069 \\
{[0.184]}\end{array}$ & $\begin{array}{c}1.061 \\
{[0.181]}\end{array}$ & $\begin{array}{c}1.218 \\
{[0.353]}\end{array}$ & $\begin{array}{c}1.195 \\
{[0.345]}\end{array}$ & $\begin{array}{c}1.061 \\
{[0.182]}\end{array}$ & $\begin{array}{c}1.211 \\
{[0.443]}\end{array}$ \\
\hline Age $=19$ & $\begin{array}{r}1.132 \\
{[0.307]} \\
\end{array}$ & $\begin{array}{r}1.124 \\
{[0.309]} \\
\end{array}$ & $\begin{array}{r}1.125 \\
{[0.307]} \\
\end{array}$ & $\begin{array}{c}1.130 \\
{[0.194]} \\
\end{array}$ & $\begin{array}{c}1.133 \\
{[0.195]} \\
\end{array}$ & $\begin{array}{c}0.993 \\
{[0.290]}\end{array}$ & $\begin{array}{c}0.961 \\
{[0.282]} \\
\end{array}$ & $\begin{array}{c}1.130 \\
{[0.194]}\end{array}$ & $\begin{array}{c}0.956 \\
{[0.362]}\end{array}$ \\
\hline \multicolumn{10}{|c|}{ B) Dependent Variable: Ever Given Birth } \\
\hline Age $=15$ & $\begin{array}{c}0.392 \\
{[0.398]}\end{array}$ & $\begin{array}{c}0.254 \\
{[0.333]}\end{array}$ & $\begin{array}{c}0.289 \\
{[0.371]}\end{array}$ & $\begin{array}{c}0.526 \\
{[0.297]}\end{array}$ & $\begin{array}{c}0.330 \\
{[0.228]}\end{array}$ & $\begin{array}{c}0.580 \\
{[0.664]}\end{array}$ & $\begin{array}{c}0.340 \\
{[0.479]}\end{array}$ & $\begin{array}{c}0.313 \\
{[0.232]}\end{array}$ & $\begin{array}{c}0.427 \\
{[0.733]}\end{array}$ \\
\hline Age $=16$ & $\begin{array}{c}0.323 * * \\
{[0.185]}\end{array}$ & $\begin{array}{c}0.210 * * \\
{[0.151]}\end{array}$ & $\begin{array}{c}0.200 * * \\
{[0.147]}\end{array}$ & $\begin{array}{c}0.517 \\
{[0.209]}\end{array}$ & $\begin{array}{c}0.385 * * \\
{[0.182]}\end{array}$ & $\begin{array}{c}0.371 \\
{[0.248]}\end{array}$ & $\begin{array}{c}0.241 * \\
{[0.182]}\end{array}$ & $\begin{array}{c}0.367 * * \\
{[0.182]}\end{array}$ & $\begin{array}{c}0.164 * \\
{[0.163]}\end{array}$ \\
\hline Age $=17$ & $\begin{array}{c}0.413 * * \\
{[0.160]}\end{array}$ & $\begin{array}{c}0.330 * * \\
{[0.149]}\end{array}$ & $\begin{array}{c}0.313 * * \\
{[0.143]}\end{array}$ & $\begin{array}{c}0.481 * * \\
{[0.140]}\end{array}$ & $\begin{array}{c}0.424 * * * \\
{[0.139]}\end{array}$ & $\begin{array}{c}0.335 * * \\
{[0.159]}\end{array}$ & $\begin{array}{c}0.231 * * * \\
{[0.123]}\end{array}$ & $\begin{array}{c}0.408 * * * \\
{[0.138]}\end{array}$ & $\begin{array}{c}0.154 * * * \\
{[0.102]}\end{array}$ \\
\hline Age $=18$ & $\begin{array}{c}1.000 \\
{[0.336]}\end{array}$ & $\begin{array}{c}0.968 \\
{[0.331]}\end{array}$ & $\begin{array}{c}0.964 \\
{[0.329]}\end{array}$ & $\begin{array}{c}0.926 \\
{[0.208]}\end{array}$ & $\begin{array}{c}0.922 \\
{[0.208]}\end{array}$ & $\begin{array}{c}0.975 \\
{[0.367]}\end{array}$ & $\begin{array}{c}0.890 \\
{[0.339]}\end{array}$ & $\begin{array}{c}0.914 \\
{[0.208]}\end{array}$ & $\begin{array}{c}0.915 \\
{[0.442]}\end{array}$ \\
\hline Age $=19$ & $\begin{array}{c}1.236 \\
{[0.345]}\end{array}$ & $\begin{array}{c}1.236 \\
{[0.351]}\end{array}$ & $\begin{array}{c}1.233 \\
{[0.348]}\end{array}$ & $\begin{array}{c}1.190 \\
{[0.219]}\end{array}$ & $\begin{array}{c}1.197 \\
{[0.220]}\end{array}$ & $\begin{array}{c}1.219 \\
{[0.388]}\end{array}$ & $\begin{array}{c}1.157 \\
{[0.369]}\end{array}$ & $\begin{array}{c}1.191 \\
{[0.220]}\end{array}$ & $\begin{array}{c}1.185 \\
{[0.484]}\end{array}$ \\
\hline
\end{tabular}

Notes: The data come from the 2013 DHS. The sample includes 48 months on each side of the donut-hole in panel (I) and 84 months on each side of the donut-hole in panel (II). The number of observations is 2,325 all cells in part (I), while it is 4,066 in all cells for ages up to 18 and 4,040 in all cells for age 19 in panel (II). The policy dummy is one when year of birth is greater than 1987. Each cell comes from a separate logistic regression of ever-married status in panel (A) and of ever-given-birth status in panel (B) at the specified age on the policy variable and the specified time trends in month and year of birth. Odds ratios and their standard errors are given as estimated parameters. The standard errors are clustered at the month-and-year-of-birth level. Statistical significance is ${ }^{* * *}$ at 1 percent level, $* *$ at 5 percent level, * at 10 percent level. 
Table 4: Policy Effect on Marriage and First-Birth by Age, Odds Ratios - Month-Year of Birth as Running Variable, 10-Year Intervals around the Donut-hole (Sample B3)

\begin{tabular}{|c|c|c|c|c|c|c|c|c|c|}
\hline \multirow[b]{2}{*}{$\begin{array}{l}\text { Degree of } \\
\text { Polynomial } \\
\end{array}$} & \multicolumn{6}{|c|}{ Single Time Trend } & \multicolumn{3}{|c|}{ Split Time Trends } \\
\hline & $\begin{array}{l}\text { One } \\
(1)\end{array}$ & $\begin{array}{l}\text { Two } \\
\text { (2) }\end{array}$ & $\begin{array}{c}\text { Three } \\
\text { (3) }\end{array}$ & $\begin{array}{l}\text { Four } \\
(4)\end{array}$ & $\begin{array}{c}\text { Five } \\
\text { (5) }\end{array}$ & $\begin{array}{l}\text { Six } \\
(6)\end{array}$ & $\begin{array}{l}\text { One } \\
(7)\end{array}$ & $\begin{array}{l}\text { Two } \\
(8)\end{array}$ & $\begin{array}{c}\text { Three } \\
(9)\end{array}$ \\
\hline \multicolumn{10}{|c|}{ A) Dependent Variable: Ever Married } \\
\hline Age $=13$ & $\begin{array}{c}0.669 \\
{[0.449]}\end{array}$ & $\begin{array}{c}0.959 \\
{[0.617]}\end{array}$ & $\begin{array}{c}1.099 \\
{[1.030]}\end{array}$ & $\begin{array}{c}1.073 \\
{[1.002]}\end{array}$ & $\begin{array}{c}0.650 \\
{[0.794]}\end{array}$ & $\begin{array}{c}0.411 \\
{[0.535]}\end{array}$ & $\begin{array}{c}0.885 \\
{[0.546]}\end{array}$ & $\begin{array}{c}0.805 \\
{[1.008]}\end{array}$ & $\begin{array}{c}0.068 \\
{[0.162]}\end{array}$ \\
\hline Age $=14$ & $\begin{array}{c}0.567 \\
{[0.280]}\end{array}$ & $\begin{array}{c}0.796 \\
{[0.387]}\end{array}$ & $\begin{array}{c}0.752 \\
{[0.515]}\end{array}$ & $\begin{array}{c}0.527 \\
{[0.396]}\end{array}$ & $\begin{array}{c}0.566 \\
{[0.535]}\end{array}$ & $\begin{array}{c}0.286 \\
{[0.304]}\end{array}$ & $\begin{array}{c}0.724 \\
{[0.342]}\end{array}$ & $\begin{array}{c}0.366 \\
{[0.354]}\end{array}$ & $\begin{array}{c}0.107 \\
{[0.180]}\end{array}$ \\
\hline Age $=15$ & $\begin{array}{c}0.440 * * * \\
{[0.130]}\end{array}$ & $\begin{array}{l}0.559^{*} \\
{[0.177]}\end{array}$ & $\begin{array}{c}0.474 * \\
{[0.195]}\end{array}$ & $\begin{array}{c}0.323 * * \\
{[0.163]}\end{array}$ & $\begin{array}{c}0.262 * * \\
{[0.151]}\end{array}$ & $\begin{array}{c}0.184 * * \\
{[0.124]}\end{array}$ & $\begin{array}{c}0.525^{* *} \\
{[0.167]}\end{array}$ & $\begin{array}{c}0.228 * * \\
{[0.142]}\end{array}$ & $\begin{array}{c}0.076 * * \\
{[0.082]}\end{array}$ \\
\hline Age $=16$ & $\begin{array}{c}0.720 \\
{[0.145]}\end{array}$ & $\begin{array}{c}0.827 \\
{[0.173]}\end{array}$ & $\begin{array}{c}0.517 * * \\
{[0.148]}\end{array}$ & $\begin{array}{c}0.346 * * * \\
{[0.110]}\end{array}$ & $\begin{array}{c}0.407 * * \\
{[0.160]}\end{array}$ & $\begin{array}{c}0.405 * * \\
{[0.168]}\end{array}$ & $\begin{array}{c}0.791 \\
{[0.165]}\end{array}$ & $\begin{array}{c}0.264 * * * \\
{[0.100]}\end{array}$ & $\begin{array}{c}0.403 \\
{[0.254]}\end{array}$ \\
\hline Age $=17$ & $\begin{array}{c}1.202 \\
{[0.193]}\end{array}$ & $\begin{array}{l}1.336^{*} \\
{[0.217]}\end{array}$ & $\begin{array}{c}1.172 \\
{[0.292]}\end{array}$ & $\begin{array}{c}0.823 \\
{[0.216]}\end{array}$ & $\begin{array}{c}1.096 \\
{[0.384]}\end{array}$ & $\begin{array}{c}1.114 \\
{[0.419]}\end{array}$ & $\begin{array}{c}1.271 \\
{[0.204]}\end{array}$ & $\begin{array}{c}0.790 \\
{[0.258]}\end{array}$ & $\begin{array}{c}1.456 \\
{[0.885]}\end{array}$ \\
\hline Age $=18$ & $\begin{array}{c}1.222 \\
{[0.172]}\end{array}$ & $\begin{array}{l}1.328^{*} \\
{[0.203]}\end{array}$ & $\begin{array}{c}1.163 \\
{[0.257]}\end{array}$ & $\begin{array}{c}0.785 \\
{[0.193]}\end{array}$ & $\begin{array}{c}0.986 \\
{[0.294]}\end{array}$ & $\begin{array}{c}1.456 \\
{[0.527]}\end{array}$ & $\begin{array}{c}1.275 \\
{[0.190]}\end{array}$ & $\begin{array}{c}0.775 \\
{[0.226]}\end{array}$ & $\begin{array}{c}1.316 \\
{[0.711]}\end{array}$ \\
\hline Age $=19$ & $\begin{array}{c}1.125 \\
{[0.156]}\end{array}$ & $\begin{array}{c}1.146 \\
{[0.184]}\end{array}$ & $\begin{array}{c}1.092 \\
{[0.235]}\end{array}$ & $\begin{array}{c}0.903 \\
{[0.252]}\end{array}$ & $\begin{array}{c}1.047 \\
{[0.322]} \\
\end{array}$ & $\begin{array}{c}1.361 \\
{[0.576]}\end{array}$ & $\begin{array}{c}1.126 \\
{[0.172]} \\
\end{array}$ & $\begin{array}{c}0.865 \\
{[0.283]}\end{array}$ & $\begin{array}{c}1.360 \\
{[0.887]} \\
\end{array}$ \\
\hline \multicolumn{10}{|c|}{ B) Dependent Variable: Ever Given Birth } \\
\hline Age $=15$ & $\begin{array}{c}0.674 \\
{[0.320]}\end{array}$ & $\begin{array}{c}0.784 \\
{[0.369]}\end{array}$ & $\begin{array}{c}0.559 \\
{[0.358]}\end{array}$ & $\begin{array}{c}0.218^{*} \\
{[0.192]}\end{array}$ & $\begin{array}{c}0.452 \\
{[0.523]}\end{array}$ & $\begin{array}{c}0.306 \\
{[0.432]}\end{array}$ & $\begin{array}{c}0.710 \\
{[0.338]}\end{array}$ & $\begin{array}{c}0.124 \\
{[0.169]}\end{array}$ & $\begin{array}{c}0.718 \\
{[1.555]}\end{array}$ \\
\hline Age $=16$ & $\begin{array}{c}0.602 \\
{[0.193]}\end{array}$ & $\begin{array}{c}0.693 \\
{[0.253]}\end{array}$ & $\begin{array}{c}0.540 \\
{[0.232]}\end{array}$ & $\begin{array}{c}0.234 * * \\
{[0.133]}\end{array}$ & $\begin{array}{c}0.362 \\
{[0.235]}\end{array}$ & $\begin{array}{c}0.205 * * \\
{[0.163]}\end{array}$ & $\begin{array}{c}0.630 \\
{[0.230]}\end{array}$ & $\begin{array}{c}0.122 * * * \\
{[0.095]}\end{array}$ & $\begin{array}{c}0.117 \\
{[0.159]}\end{array}$ \\
\hline Age $=17$ & $\begin{array}{c}0.756 \\
{[0.167]}\end{array}$ & $\begin{array}{c}0.708 \\
{[0.199]}\end{array}$ & $\begin{array}{c}0.513 * * \\
{[0.160]}\end{array}$ & $\begin{array}{c}0.213 * * * \\
{[0.095]}\end{array}$ & $\begin{array}{c}0.262 * * * \\
{[0.117]}\end{array}$ & $\begin{array}{c}0.191 * * * \\
{[0.110]}\end{array}$ & $\begin{array}{c}0.679 \\
{[0.189]}\end{array}$ & $\begin{array}{c}0.126 * * * \\
{[0.070]}\end{array}$ & $\begin{array}{c}0.072 * * * \\
{[0.067]}\end{array}$ \\
\hline Age $=18$ & $\begin{array}{c}1.056 \\
{[0.189]}\end{array}$ & $\begin{array}{c}1.224 \\
{[0.247]}\end{array}$ & $\begin{array}{c}1.078 \\
{[0.277]}\end{array}$ & $\begin{array}{c}0.578 * \\
{[0.189]}\end{array}$ & $\begin{array}{c}0.761 \\
{[0.274]}\end{array}$ & $\begin{array}{c}1.196 \\
{[0.572]}\end{array}$ & $\begin{array}{c}1.136 \\
{[0.222]}\end{array}$ & $\begin{array}{c}0.507 * \\
{[0.192]}\end{array}$ & $\begin{array}{c}1.210 \\
{[0.837]}\end{array}$ \\
\hline Age $=19$ & $\begin{array}{c}1.122 \\
{[0.155]}\end{array}$ & $\begin{array}{c}1.213 \\
{[0.209]}\end{array}$ & $\begin{array}{c}1.394 \\
{[0.283]}\end{array}$ & $\begin{array}{c}1.022 \\
{[0.302]}\end{array}$ & $\begin{array}{c}1.040 \\
{[0.311]}\end{array}$ & $\begin{array}{c}1.620 \\
{[0.700]}\end{array}$ & $\begin{array}{c}1.142 \\
{[0.186]}\end{array}$ & $\begin{array}{c}1.114 \\
{[0.372]}\end{array}$ & $\begin{array}{c}1.129 \\
{[0.755]}\end{array}$ \\
\hline
\end{tabular}

Notes: The data come from the 2013 DHS. The sample includes 10 years on each side of the donut-hole. The number of observations is 5,946 in all cells for all ages up to $15,5,921$ for age 16, 5,589 for age 17, 5,273 for age 18, and 4,970 for age 19. The policy dummy is one when year of birth is greater than 1987. Each cell comes from a separate logistic regression of ever-married status in panel (A) and of ever-given-birth status in panel (B) at the specified age on the policy variable and the specified time trends in month and year of birth. Odds ratios and their standard errors are given as estimated parameters. The standard errors are clustered at the month-and-year-of-birth level. Statistical significance is *** at 1 percent level, ** at 5 percent level, $*$ at 10 percent level. 
Table 5: Policy Effect on Predicted Fractions of Being Ever Married and Ever Giving Birth

by Age

\begin{tabular}{|c|c|c|c|c|c|c|c|c|}
\hline \multirow[b]{2}{*}{ Age } & \multicolumn{4}{|c|}{ I) 4-Year Intervals } & \multicolumn{4}{|c|}{ II) 7-Year Intervals } \\
\hline & Baseline & Policy & Diff. & $\%$ Fall & Baseline & Policy & Diff. & $\%$ Fall \\
\hline \multicolumn{9}{|c|}{ A) Ever Married } \\
\hline 13 & 0.010 & 0.004 & 0.006 & 60.0 & 0.010 & 0.010 & 0.000 & 0.0 \\
\hline 14 & 0.027 & 0.008 & 0.019 & 70.4 & 0.021 & 0.016 & 0.005 & 23.8 \\
\hline 15 & 0.071 & 0.021 & $0.050 * *$ & 70.4 & 0.065 & 0.027 & $0.038 * *$ & 58.5 \\
\hline 16 & 0.117 & 0.060 & $0.057 * *$ & 48.7 & 0.119 & 0.062 & $0.057 * *$ & 47.9 \\
\hline 17 & 0.125 & 0.158 & -0.033 & -26.4 & 0.157 & 0.160 & -0.003 & -1.9 \\
\hline 18 & 0.229 & 0.250 & -0.021 & -9.2 & 0.242 & 0.253 & -0.011 & -4.5 \\
\hline 19 & 0.313 & 0.338 & -0.025 & -8.0 & 0.331 & 0.359 & -0.028 & -8.5 \\
\hline \multicolumn{9}{|c|}{ B) Ever Given Birth } \\
\hline 15 & 0.013 & 0.004 & 0.009 & 69.2 & 0.012 & 0.004 & 0.008 & 66.7 \\
\hline 16 & 0.035 & 0.007 & $0.028 * *$ & 80.0 & 0.034 & 0.013 & $0.021 * *$ & 61.8 \\
\hline 17 & 0.074 & 0.025 & $0.049 * * *$ & 66.2 & 0.081 & 0.035 & $0.046 * * *$ & 56.8 \\
\hline 18 & 0.112 & 0.108 & 0.004 & 3.6 & 0.132 & 0.122 & 0.010 & 7.6 \\
\hline 19 & 0.184 & 0.217 & -0.033 & -17.9 & 0.193 & 0.222 & -0.029 & -15.0 \\
\hline
\end{tabular}

Notes: Predictions are based on the estimates given in columns (3) and (8) of Table 3 (specifications with split time trends in the form of linear polynomials). Predictions are given for someone born between December 1986 and January 1987. Statistically significant *** at 1 percent level, ** at 5 percent level, $*$ at 10 percent level. 
Table 6: Policy Effect on the Time to First Birth after Marriage, OLS Results

\begin{tabular}{|c|c|c|c|c|c|c|c|c|c|c|}
\hline \multirow[b]{2}{*}{ Degree of Polynomial } & \multicolumn{6}{|c|}{ Single Time Trend } & \multicolumn{3}{|c|}{ Split Time Trends } & \multirow[b]{2}{*}{ Obs. } \\
\hline & $\begin{array}{l}\text { One } \\
(1)\end{array}$ & $\begin{array}{l}\text { Two } \\
(2)\end{array}$ & $\begin{array}{l}\text { Three } \\
(3)\end{array}$ & $\begin{array}{l}\text { Four } \\
(4)\end{array}$ & $\begin{array}{l}\text { Five } \\
(5)\end{array}$ & $\begin{array}{l}\text { Six } \\
(6)\end{array}$ & $\begin{array}{l}\text { One } \\
(7)\end{array}$ & $\begin{array}{l}\text { Two } \\
(8)\end{array}$ & $\begin{array}{l}\text { Three } \\
(9)\end{array}$ & \\
\hline \multicolumn{11}{|c|}{ A) With age at marriage dummies } \\
\hline $\begin{array}{l}\text { Year of Birth } \\
1959-1998\end{array}$ & $\begin{array}{c}-3.457 * * * \\
{[0.630]}\end{array}$ & $\begin{array}{l}-1.022 \\
{[0.761]}\end{array}$ & $\begin{array}{l}-0.008 \\
{[0.575]}\end{array}$ & $\begin{array}{l}-0.070 \\
{[0.668]}\end{array}$ & -- & -- & $\begin{array}{c}-1.195 * * \\
{[0.440]}\end{array}$ & $\begin{array}{c}-0.485 \\
{[0.738]}\end{array}$ & -- & 12,635 \\
\hline $\begin{array}{l}\text { Month-Year of Birth } \\
\text { 4-year intervals }\end{array}$ & $\begin{array}{c}1.593 \\
{[1.786]}\end{array}$ & $\begin{array}{c}1.735 \\
{[1.750]}\end{array}$ & $\begin{array}{l}-- \\
--\end{array}$ & $\begin{array}{l}-- \\
--\end{array}$ & $\begin{array}{l}-- \\
--\end{array}$ & $\begin{array}{l}-- \\
--\end{array}$ & $\begin{array}{c}1.681 \\
{[1.749]}\end{array}$ & -- & $\begin{array}{l}-- \\
--\end{array}$ & 1,514 \\
\hline $\begin{array}{l}\text { Month-Year of Birth } \\
\text { 7-year intervals }\end{array}$ & $\begin{array}{l}-1.290 \\
{[1.419]}\end{array}$ & $\begin{array}{l}-0.270 \\
{[1.321]}\end{array}$ & $\begin{array}{c}1.887 \\
{[2.144]}\end{array}$ & $\begin{array}{c}1.551 \\
{[2.069]}\end{array}$ & $\begin{array}{l}-- \\
--\end{array}$ & $\begin{array}{l}-- \\
--\end{array}$ & $\begin{array}{l}-0.556 \\
{[1.332]}\end{array}$ & $\begin{array}{l}2.422 * * \\
{[0.899]}\end{array}$ & $\begin{array}{l}-- \\
--\end{array}$ & 2,516 \\
\hline $\begin{array}{l}\text { Month-Year of Birth } \\
10 \text {-year intervals }\end{array}$ & $\begin{array}{l}-2.229^{*} \\
{[1.271]}\end{array}$ & $\begin{array}{c}-0.113 \\
{[1.232]}\end{array}$ & $\begin{array}{c}0.274 \\
{[1.795]}\end{array}$ & $\begin{array}{c}0.898 \\
{[1.880]}\end{array}$ & $\begin{array}{c}1.999 \\
{[2.425]}\end{array}$ & $\begin{array}{c}3.088 \\
{[2.499]}\end{array}$ & $\begin{array}{c}-0.703 \\
{[1.238]}\end{array}$ & $\begin{array}{c}0.776 \\
{[1.112]}\end{array}$ & $\begin{array}{c}4.389 * * \\
{[1.739]}\end{array}$ & 3,394 \\
\hline
\end{tabular}

B) Without age at marriage dummies

\begin{tabular}{|c|c|c|c|c|c|c|c|c|c|c|}
\hline $\begin{array}{l}\text { Year of Birth } \\
1959-1998\end{array}$ & $\begin{array}{c}-3.009 * * * \\
{[0.554]}\end{array}$ & $\begin{array}{l}-0.862 \\
{[0.733]}\end{array}$ & $\begin{array}{l}-0.225 \\
{[0.604]}\end{array}$ & $\begin{array}{l}-0.276 \\
{[0.714]}\end{array}$ & $\begin{array}{l}-- \\
--\end{array}$ & $\begin{array}{l}-- \\
--\end{array}$ & $\begin{array}{c}-1.413^{* * *} \\
{[0.410]}\end{array}$ & $\begin{array}{c}-0.671 \\
{[0.677]}\end{array}$ & $\begin{array}{l}-- \\
--\end{array}$ & 12,635 \\
\hline $\begin{array}{l}\text { Month-Year of Birth } \\
\text { 4-year intervals }\end{array}$ & $\begin{array}{c}1.351 \\
{[1.876]}\end{array}$ & $\begin{array}{c}1.090 \\
{[1.828]}\end{array}$ & $\begin{array}{l}-- \\
--\end{array}$ & $\begin{array}{l}-- \\
--\end{array}$ & $\begin{array}{l}-- \\
--\end{array}$ & $\begin{array}{l}-- \\
--\end{array}$ & $\begin{array}{c}1.051 \\
{[1.827]}\end{array}$ & $\begin{array}{l}-- \\
--\end{array}$ & $\begin{array}{l}-- \\
--\end{array}$ & 1,514 \\
\hline $\begin{array}{l}\text { Month-Year of Birth } \\
7 \text {-year intervals }\end{array}$ & $\begin{array}{l}-1.530 \\
{[1.453]}\end{array}$ & $\begin{array}{c}-0.911 \\
{[1.336]}\end{array}$ & $\begin{array}{c}1.775 \\
{[2.245]}\end{array}$ & $\begin{array}{c}0.658 \\
{[2.113]}\end{array}$ & $\begin{array}{l}-- \\
--\end{array}$ & $\begin{array}{l}-- \\
--\end{array}$ & $\begin{array}{l}-1.231 \\
{[1.353]}\end{array}$ & $\begin{array}{l}1.761^{*} \\
{[0.944]}\end{array}$ & $\begin{array}{l}-- \\
--\end{array}$ & 2,516 \\
\hline $\begin{array}{l}\text { Month-Year of Birth } \\
10 \text {-year intervals }\end{array}$ & $\begin{array}{c}-2.832 * * \\
{[1.245]}\end{array}$ & $\begin{array}{c}-1.143 \\
{[1.194]}\end{array}$ & $\begin{array}{l}-0.061 \\
{[1.827]}\end{array}$ & $\begin{array}{l}-0.149 \\
{[1.859]}\end{array}$ & $\begin{array}{c}1.233 \\
{[2.492]}\end{array}$ & $\begin{array}{c}2.108 \\
{[2.490]}\end{array}$ & $\begin{array}{c}-1.883 \\
{[1.196]}\end{array}$ & $\begin{array}{l}-0.161 \\
{[1.013]}\end{array}$ & $\begin{array}{l}3.832^{*} \\
{[1.867]}\end{array}$ & 3,394 \\
\hline
\end{tabular}

Notes: The sample includes observations from both the 2008 and 2013 DHS in the analysis by year of birth, but only from the 2013 DHS in the analysis by month-year of birth. The sample is restricted to ages 17 and above because the policy has no effect on marriage status after age 17. In all analyses, 1986 and 1987 birth cohorts are excluded. The policy dummy is one when year of birth is greater than 1987. Each cell comes from a separate OLS regresion of the time to first-birth after marriage (in months) on the policy dummy and the specified time trends. Standard errors are clustered at the yearof-birth level when the running variable is year of birth and at the month-year of birth level when the running variable is month-year of birth. Statistical significance is $* * *$ at 1 percent level, $* *$ at 5 percent level, $*$ at 10 percent level. 
Table 7: Policy Effect on Number of Children Ever Born - Month-Year of Birth as Running Variable, 4-Year and 7-Year Intervals around the Donut-hole, OLS Results

\begin{tabular}{|c|c|c|c|c|c|c|c|c|c|}
\hline \multirow[b]{3}{*}{$\begin{array}{l}\text { Degree of } \\
\text { Polynomial }\end{array}$} & \multicolumn{3}{|c|}{ II) 4-Year Intervals } & \multicolumn{6}{|c|}{ II) 7-Year Intervals } \\
\hline & \multicolumn{2}{|c|}{ Single Time Trend } & \multirow{2}{*}{$\begin{array}{c}\text { Split } \\
\text { One } \\
(3)\end{array}$} & \multicolumn{4}{|c|}{ Single Time Trend } & \multicolumn{2}{|c|}{ Split Time Trends } \\
\hline & $\begin{array}{l}\text { One } \\
(1)\end{array}$ & $\begin{array}{l}\text { Two } \\
(2) \\
\end{array}$ & & $\begin{array}{l}\text { One } \\
(4)\end{array}$ & $\begin{array}{l}\text { Two } \\
(5) \\
\end{array}$ & $\begin{array}{c}\text { Three } \\
(6)\end{array}$ & $\begin{array}{c}\text { Four } \\
(7) \\
\end{array}$ & $\begin{array}{l}\text { One } \\
(8)\end{array}$ & $\begin{array}{l}\text { Two } \\
(9)\end{array}$ \\
\hline Age $=15$ & $\begin{array}{c}0.001 \\
{[0.008]}\end{array}$ & $\begin{array}{c}0.001 \\
{[0.008]}\end{array}$ & $\begin{array}{c}0.001 \\
{[0.008]}\end{array}$ & $\begin{array}{c}0.006 \\
{[0.005]}\end{array}$ & $\begin{array}{c}0.006 \\
{[0.005]}\end{array}$ & $\begin{array}{c}0.005 \\
{[0.009]}\end{array}$ & $\begin{array}{c}0.004 \\
{[0.009]}\end{array}$ & $\begin{array}{c}0.006 \\
{[0.005]}\end{array}$ & $\begin{array}{c}0.003 \\
{[0.011]}\end{array}$ \\
\hline Age $=16$ & $\begin{array}{c}-0.009 \\
{[0.011]}\end{array}$ & $\begin{array}{c}-0.009 \\
{[0.011]}\end{array}$ & $\begin{array}{c}-0.009 \\
{[0.011]}\end{array}$ & $\begin{array}{c}-0.006 \\
{[0.008]}\end{array}$ & $\begin{array}{c}-0.006 \\
{[0.008]}\end{array}$ & $\begin{array}{c}-0.002 \\
{[0.014]}\end{array}$ & $\begin{array}{c}-0.003 \\
{[0.014]}\end{array}$ & $\begin{array}{c}-0.006 \\
{[0.008]}\end{array}$ & $\begin{array}{c}-0.003 \\
{[0.018]}\end{array}$ \\
\hline Age $=17$ & $\begin{array}{c}-0.029 \\
{[0.019]}\end{array}$ & $\begin{array}{l}-0.031^{*} \\
{[0.018]}\end{array}$ & $\begin{array}{c}-0.030 \\
{[0.018]}\end{array}$ & $\begin{array}{c}-0.016 \\
{[0.015]}\end{array}$ & $\begin{array}{c}-0.017 \\
{[0.015]}\end{array}$ & $\begin{array}{c}-0.014 \\
{[0.023]}\end{array}$ & $\begin{array}{c}-0.015 \\
{[0.022]}\end{array}$ & $\begin{array}{c}-0.016 \\
{[0.014]}\end{array}$ & $\begin{array}{c}-0.017 \\
{[0.028]}\end{array}$ \\
\hline Age $=18$ & $\begin{array}{c}-0.070 * * \\
{[0.031]}\end{array}$ & $\begin{array}{c}-0.071 * * \\
{[0.029]}\end{array}$ & $\begin{array}{c}-0.070 * * \\
{[0.029]}\end{array}$ & $\begin{array}{c}-0.052 * * \\
{[0.025]}\end{array}$ & $\begin{array}{c}-0.052 * * \\
{[0.024]}\end{array}$ & $\begin{array}{c}-0.072 * \\
{[0.038]}\end{array}$ & $\begin{array}{c}-0.074 * * \\
{[0.036]}\end{array}$ & $\begin{array}{c}-0.052 * * \\
{[0.024]}\end{array}$ & $\begin{array}{c}-0.085^{*} \\
{[0.045]}\end{array}$ \\
\hline Age $=19$ & $\begin{array}{c}-0.003 \\
{[0.049]}\end{array}$ & $\begin{array}{c}-0.005 \\
{[0.047]}\end{array}$ & $\begin{array}{c}-0.004 \\
{[0.046]}\end{array}$ & $\begin{array}{c}-0.018 \\
{[0.034]}\end{array}$ & $\begin{array}{c}-0.019 \\
{[0.034]}\end{array}$ & $\begin{array}{c}0.007 \\
{[0.056]}\end{array}$ & $\begin{array}{c}0.002 \\
{[0.052]}\end{array}$ & $\begin{array}{c}-0.018 \\
{[0.033]}\end{array}$ & $\begin{array}{c}0.013 \\
{[0.065]}\end{array}$ \\
\hline Age $=20$ & $\begin{array}{c}0.023 \\
{[0.061]}\end{array}$ & $\begin{array}{c}0.022 \\
{[0.060]}\end{array}$ & $\begin{array}{c}0.022 \\
{[0.059]}\end{array}$ & $\begin{array}{c}-0.004 \\
{[0.046]}\end{array}$ & $\begin{array}{c}-0.016 \\
{[0.046]}\end{array}$ & $\begin{array}{c}0.082 \\
{[0.076]}\end{array}$ & $\begin{array}{c}0.060 \\
{[0.074]}\end{array}$ & $\begin{array}{c}-0.016 \\
{[0.045]}\end{array}$ & $\begin{array}{c}0.076 \\
{[0.092]}\end{array}$ \\
\hline
\end{tabular}

Notes: The data come from the 2013 DHS. The sample includes 48 months on each side of the donut-hole in panel (I) and 84 months on each side of the donut-hole in panel (II) (except for ages 19 and 20 in panel II, where there are 72 and 60 months, respectively, on the righthand-side of the donut-hole). The number of observations is 2,324 in all cells in part (I), while it is 4,061 in all cells for ages up to 18 , 4,035 in all cells for age 19, and 3,750 in all cells for age 20 in panel (II). The policy dummy is one when year of birth is greater than 1987. Each cell comes from a separate OLS regression of the number of children born by the specified age on the policy variable and the specified time trends in month and year of birth. The standard errors are clustered at the month-and-year-of-birth level. Statistical significance is $* * *$ at 1 percent level, $* *$ at 5 percent level, $*$ at 10 percent level. 


\section{ONLINE APPENDIX (Not for Print Publication)}

\section{APPENDIX A: SUPPLEMENTARY FIGURES AND TABLES}

Figure A1: Number of Students in Basic Education (Grades 1 to 8)
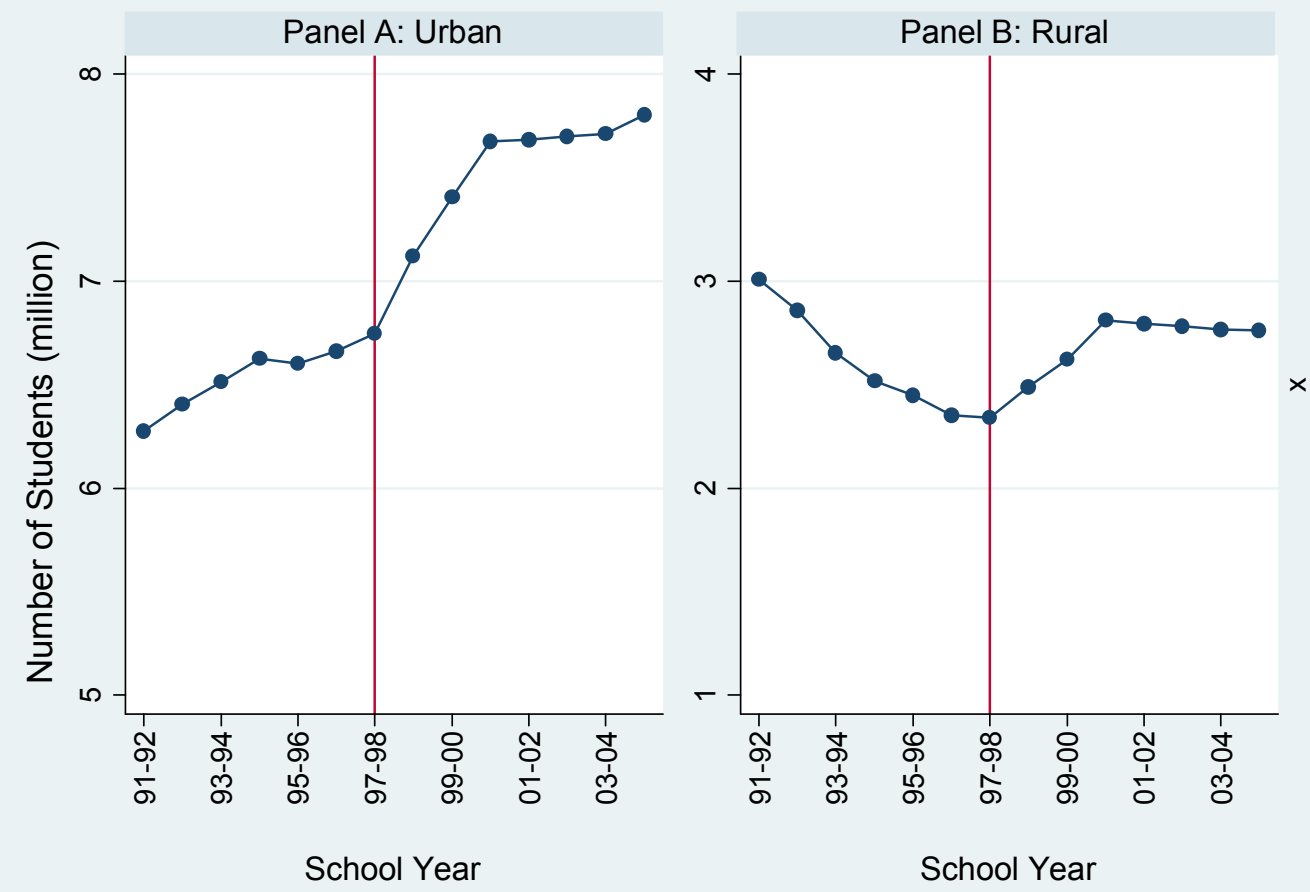

Source: Turkish Statistical Institute (1992-2005) 


\section{Figure A2: Testing whether the Cut-off Value for the Running Variable is Related to Interventions other than the 1997 Education Reform}
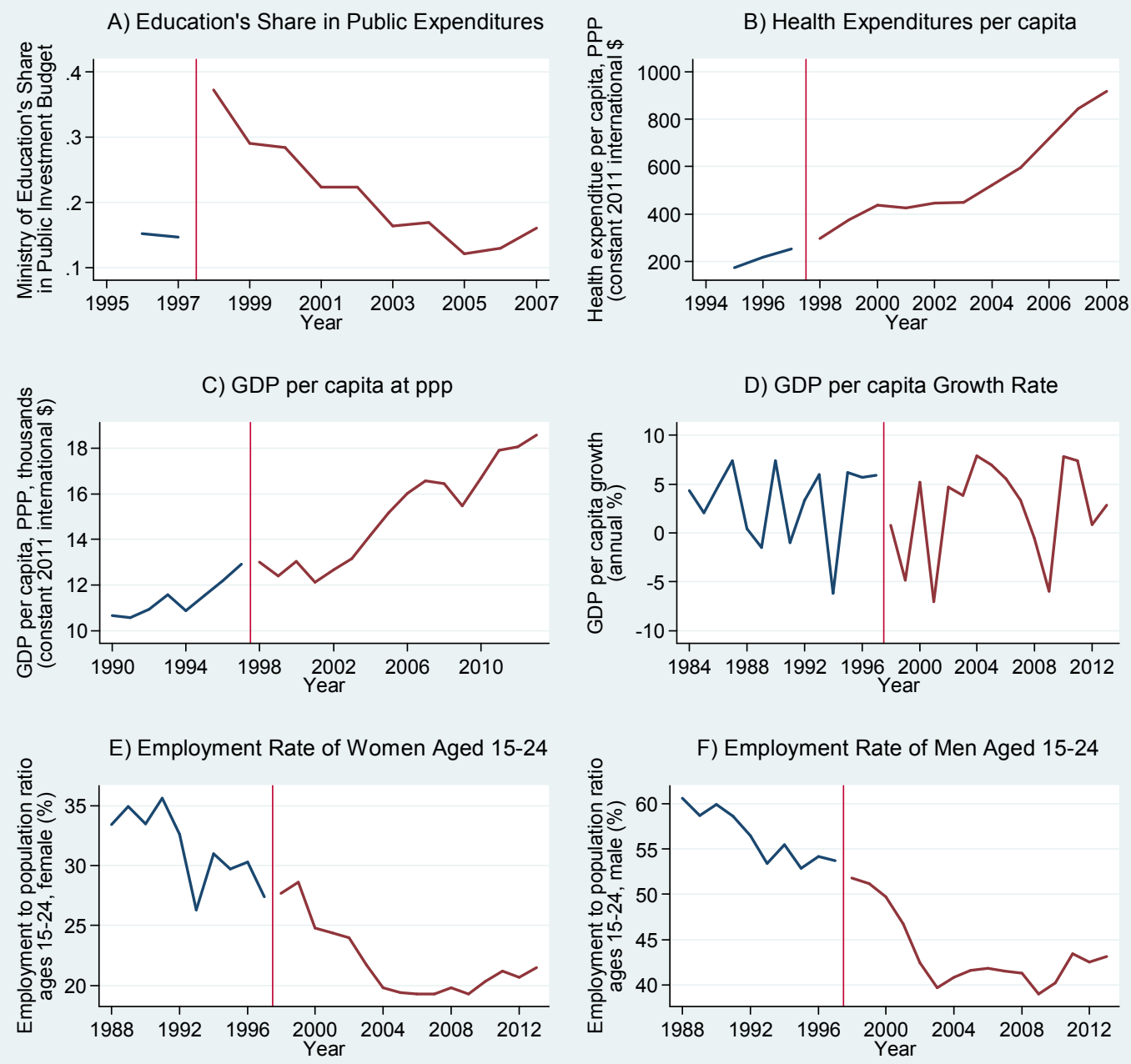

G) Infant Mortality Rate

H) Under-5 Mortality Rate
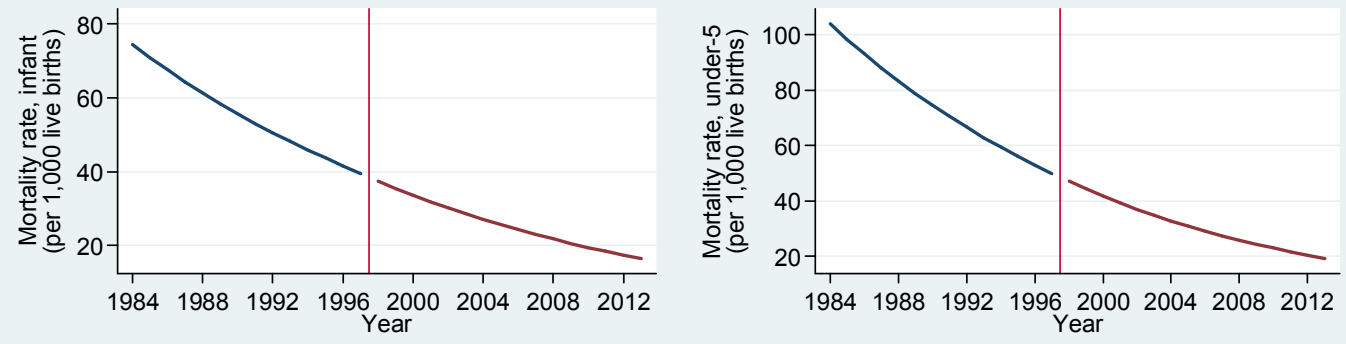

Source: Turkish Statistical Institute (2006), World Development Indicators (2015). 
Figure A3: Time to First Birth after Marriage

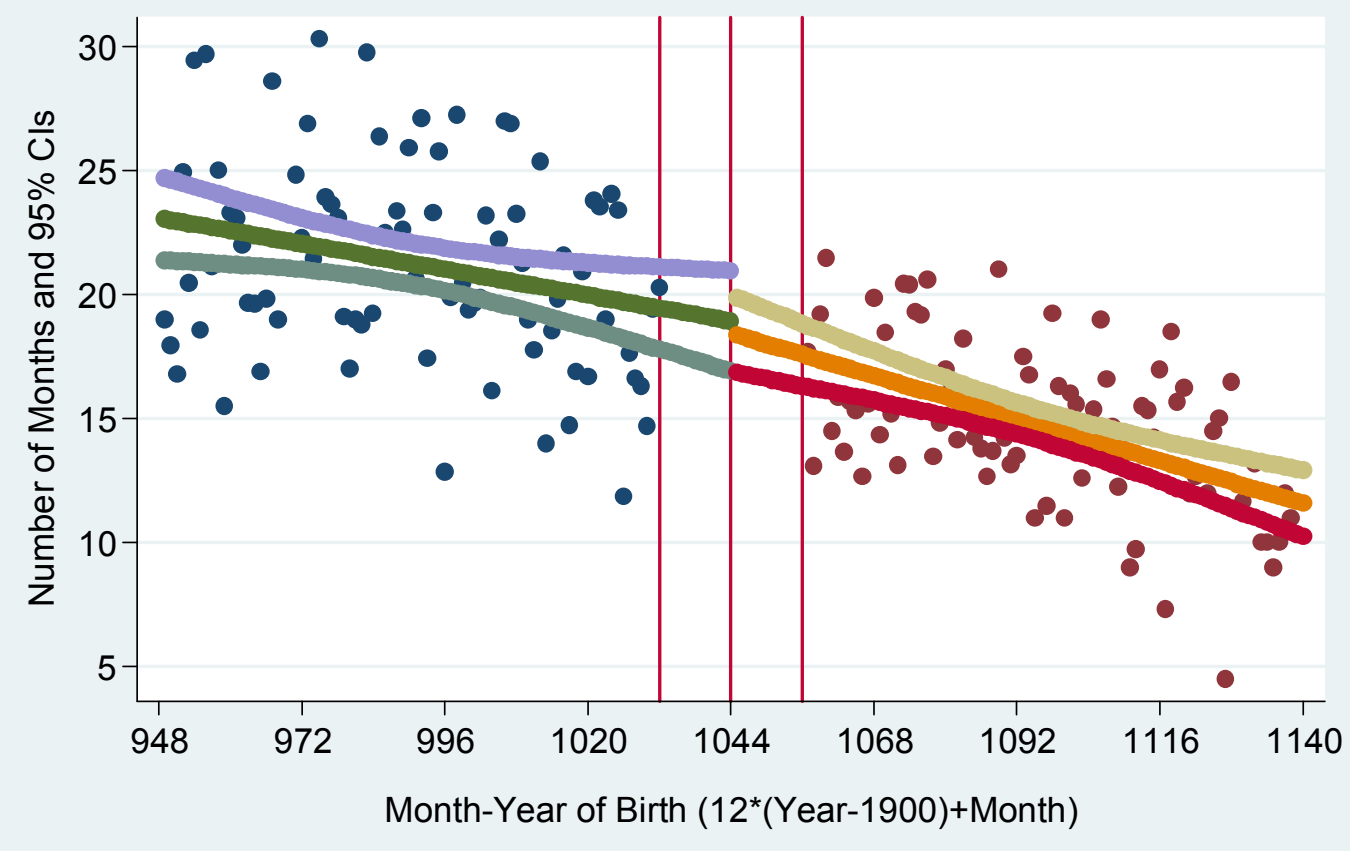

Notes: a) The sample includes women aged 17 and above in the $2013 \mathrm{DHS}$.

b) 1986 and 1987 birth cohorts are excluded due to the fuzziness of treatment status.

c) Linear polynomials are fit before and after the cut-off. 
Table A1: Descriptive Statistics I: Person-Level Characteristics for Sample (A)

\begin{tabular}{lrrrrr}
\hline & Mean & S.D. & Min. & Max. & No. Obs. \\
\hline Year of Birth & 1979.78 & 10.32 & 1959 & 1998 & 21,862 \\
Age & 30.69 & 10.07 & 15 & 49 & 21,862 \\
Policy & 0.31 & 0.46 & 0 & 1 & 21,862 \\
Years of Schooling & 6.99 & 4.26 & 0 & 21 & 21,845 \\
Ever Married & 0.69 & 0.46 & 0 & 1 & 21,862 \\
Age at Marriage & 21.47 & 6.52 & 7 & 49 & 15,112 \\
Age at First-Birth & 21.51 & 4.19 & 10 & 44 & 12,644 \\
Time to First-Birth after Marriage (months) & 21.11 & 21.47 & 0 & 337 & 12,644 \\
Location of Birth Characteristics & & & & & \\
Rural & 0.442 & 0.497 & 0 & 1 & 21,837 \\
NUTS-1 Level Region & & & & & \\
$\quad$ Region 1 & 0.044 & 0.204 & 0 & 1 & 21,849 \\
$\quad$ Region 2 & 0.042 & 0.200 & 0 & 1 & 21,849 \\
$\quad$ Region 3 & 0.061 & 0.239 & 0 & 1 & 21,849 \\
$\quad$ Region 4 & 0.049 & 0.215 & 0 & 1 & 21,849 \\
$\quad$ Region 5 & 0.065 & 0.246 & 0 & 1 & 21,849 \\
$\quad$ Region 6 & 0.124 & 0.329 & 0 & 1 & 21,849 \\
$\quad$ Region 7 & 0.080 & 0.271 & 0 & 1 & 21,849 \\
$\quad$ Region 8 & 0.101 & 0.301 & 0 & 1 & 21,849 \\
$\quad$ Region 9 & 0.081 & 0.273 & 0 & 1 & 21,849 \\
$\quad$ Region 10 & 0.094 & 0.291 & 0 & 1 & 21,849 \\
$\quad$ Region 11 & 0.101 & 0.301 & 0 & 1 & 21,849 \\
$\quad$ Region 12 & 0.146 & 0.353 & 0 & 1 & 21,849 \\
$\quad$ Foreign Born & 0.015 & 0.120 & 0 & 1 & 21,849 \\
\hline
\end{tabular}

Notes: The sample is drawn from 15- to 49-year-old individuals in the 2008 and 2013 DHS surveys. The sample excludes the 1986 and 1987 birth cohorts. 
Table A2: Descriptive Statistics II: Person-Level Characteristics for Samples (B1) - (B3)

\begin{tabular}{|c|c|c|c|c|c|c|c|c|c|c|c|c|c|c|c|}
\hline & \multicolumn{5}{|c|}{ A) Sample B1 } & \multicolumn{5}{|c|}{ B) Sample B2 } & \multicolumn{5}{|c|}{ C) Sample B3 } \\
\hline & Mean & S.D. & Min. & Max. & Obs. & Mean & S.D. & Min. & Max. & Obs. & Mean & S.D. & Min. & Max. & Obs. \\
\hline Year of Birth & 1986.4 & 3.20 & 1982 & 1991 & 2,325 & 1986.3 & 4.91 & 1979 & 1994 & 4,066 & 1986.4 & 6.71 & 1976 & 1997 & 5,946 \\
\hline Age & 26.51 & 3.21 & 21 & 31 & 2,325 & 26.61 & 4.91 & 18 & 34 & 4,066 & 26.50 & 6.71 & 15 & 37 & 5,946 \\
\hline Policy & 0.49 & 0.50 & 0 & 1 & 2,325 & 0.48 & 0.50 & 0 & 1 & 4,066 & 0.49 & 0.50 & 0 & 1 & 5,946 \\
\hline Years of Schooling & 8.16 & 4.56 & 0 & 21 & 2,325 & 8.13 & 4.39 & 0 & 21 & 4,065 & 8.08 & 4.16 & 0 & 21 & 5,945 \\
\hline Ever Married & 0.76 & 0.43 & 0 & 1 & 2,325 & 0.72 & 0.45 & 0 & 1 & 4,066 & 0.65 & 0.48 & 0 & 1 & 5,946 \\
\hline Age at Marriage & 20.11 & 3.35 & 10 & 31 & 1,775 & 20.08 & 3.62 & 10 & 34 & 2,924 & 20.15 & 3.84 & 10 & 36 & 3,867 \\
\hline Age at First-Birth & 21.28 & 3.23 & 12 & 31 & 1,514 & 21.44 & 3.66 & 12 & 34 & 2,516 & 21.62 & 3.95 & 12 & 36 & 3,395 \\
\hline \multicolumn{16}{|l|}{ Time to First-Birth after } \\
\hline Marriage (months) & 20.06 & 16.51 & 0 & 227 & 1,514 & 20.59 & 17.29 & 0 & 227 & 2,516 & 21.16 & 19.15 & 0 & 227 & 3,395 \\
\hline \multicolumn{16}{|l|}{ Location of Birth } \\
\hline $\begin{array}{l}\text { Rural } \\
\text { NUTS-1 Region }\end{array}$ & \multicolumn{14}{|c|}{ NUTS-1 Region } & 5,939 \\
\hline Region 1 & 0.052 & 0.222 & 0 & 1 & 2324 & 0.050 & 0.218 & 0 & 1 & 4063 & 0.051 & 0.221 & 0 & 1 & 5,942 \\
\hline Region 2 & 0.032 & 0.177 & 0 & 1 & 2324 & 0.030 & 0.171 & 0 & 1 & 4063 & 0.034 & 0.182 & 0 & 1 & 5,942 \\
\hline Region 3 & 0.063 & 0.243 & 0 & 1 & 2324 & 0.065 & 0.247 & 0 & 1 & 4063 & 0.063 & 0.243 & 0 & 1 & 5,942 \\
\hline Region 4 & 0.043 & 0.202 & 0 & 1 & 2324 & 0.044 & 0.206 & 0 & 1 & 4063 & 0.048 & 0.213 & 0 & 1 & 5,942 \\
\hline Region 5 & 0.064 & 0.244 & 0 & 1 & 2324 & 0.063 & 0.243 & 0 & 1 & 4063 & 0.064 & 0.245 & 0 & 1 & 5,942 \\
\hline Region 6 & 0.121 & 0.327 & 0 & 1 & 2324 & 0.120 & 0.325 & 0 & 1 & 4063 & 0.121 & 0.327 & 0 & 1 & 5,942 \\
\hline Region 7 & 0.077 & 0.266 & 0 & 1 & 2324 & 0.082 & 0.274 & 0 & 1 & 4063 & 0.076 & 0.265 & 0 & 1 & 5,942 \\
\hline Region 8 & 0.094 & 0.292 & 0 & 1 & 2324 & 0.093 & 0.291 & 0 & 1 & 4063 & 0.093 & 0.291 & 0 & 1 & 5,942 \\
\hline Region 9 & 0.080 & 0.272 & 0 & 1 & 2324 & 0.083 & 0.276 & 0 & 1 & 4063 & 0.085 & 0.279 & 0 & 1 & 5,942 \\
\hline Region 10 & 0.100 & 0.300 & 0 & 1 & 2324 & 0.101 & 0.301 & 0 & 1 & 4063 & 0.097 & 0.296 & 0 & 1 & 5,942 \\
\hline Region 11 & 0.100 & 0.300 & 0 & 1 & 2324 & 0.102 & 0.303 & 0 & 1 & 4063 & 0.102 & 0.302 & 0 & 1 & 5,942 \\
\hline Region 12 & 0.157 & 0.364 & 0 & 1 & 2324 & 0.149 & 0.356 & 0 & 1 & 4063 & 0.148 & 0.356 & 0 & 1 & 5,942 \\
\hline Foreign Born & 0.018 & 0.133 & 0 & 1 & 2324 & 0.018 & 0.134 & 0 & 1 & 4063 & 0.017 & 0.130 & 0 & 1 & 5,942 \\
\hline \multicolumn{16}{|l|}{ Month of Birth } \\
\hline January & 0.117 & 0.321 & 0 & 1 & 2325 & 0.109 & 0.311 & 0 & 1 & 4066 & 0.108 & 0.311 & 0 & 1 & 5,946 \\
\hline February & 0.083 & 0.276 & 0 & 1 & 2325 & 0.080 & 0.271 & 0 & 1 & 4066 & 0.082 & 0.275 & 0 & 1 & 5,946 \\
\hline March & 0.092 & 0.289 & 0 & 1 & 2325 & 0.092 & 0.289 & 0 & 1 & 4066 & 0.090 & 0.287 & 0 & 1 & 5,946 \\
\hline April & 0.092 & 0.290 & 0 & 1 & 2325 & 0.093 & 0.290 & 0 & 1 & 4066 & 0.091 & 0.288 & 0 & 1 & 5,946 \\
\hline May & 0.072 & 0.259 & 0 & 1 & 2325 & 0.077 & 0.266 & 0 & 1 & 4066 & 0.082 & 0.274 & 0 & 1 & 5,946 \\
\hline June & 0.087 & 0.282 & 0 & 1 & 2325 & 0.086 & 0.281 & 0 & 1 & 4066 & 0.084 & 0.277 & 0 & 1 & 5,946 \\
\hline July & 0.077 & 0.267 & 0 & 1 & 2325 & 0.074 & 0.262 & 0 & 1 & 4066 & 0.076 & 0.265 & 0 & 1 & 5,946 \\
\hline August & 0.079 & 0.270 & 0 & 1 & 2325 & 0.090 & 0.286 & 0 & 1 & 4066 & 0.089 & 0.284 & 0 & 1 & 5,946 \\
\hline September & 0.092 & 0.289 & 0 & 1 & 2325 & 0.088 & 0.283 & 0 & 1 & 4066 & 0.088 & 0.284 & 0 & 1 & 5,946 \\
\hline October & 0.089 & 0.285 & 0 & 1 & 2325 & 0.090 & 0.286 & 0 & 1 & 4066 & 0.085 & 0.279 & 0 & 1 & 5,946 \\
\hline November & 0.062 & 0.240 & 0 & 1 & 2325 & 0.064 & 0.245 & 0 & 1 & 4066 & 0.064 & 0.244 & 0 & 1 & 5,946 \\
\hline December & 0.058 & 0.233 & 0 & 1 & 2325 & 0.058 & 0.234 & 0 & 1 & 4066 & 0.060 & 0.238 & 0 & 1 & 5,946 \\
\hline
\end{tabular}

Notes: The samples are drawn from 15- to 49-year-old individuals in the 2013 DHS survey. All samples exclude the 1986 and 1987 birth cohorts. 
Table A3: Check of a Policy Effect on Other Covariates at the Cut-off, Odds Ratios

\begin{tabular}{|c|c|c|c|c|c|c|c|c|c|}
\hline \multirow[b]{3}{*}{$\begin{array}{l}\text { Degree of } \\
\text { Polynomial } \\
\end{array}$} & \multicolumn{3}{|c|}{ A) 4-year Intervals } & \multicolumn{6}{|c|}{ B) 7-year Intervals } \\
\hline & \multicolumn{2}{|c|}{ Single Time Trend } & \multirow{2}{*}{$\begin{array}{c}\text { Split Time } \\
\text { Trends }\end{array}$} & \multicolumn{4}{|c|}{ Single Time Trend } & \multicolumn{2}{|c|}{ Split Time Trends } \\
\hline & $\begin{array}{c}\text { One } \\
(1)\end{array}$ & $\begin{array}{l}\text { Two } \\
(2)\end{array}$ & & $\begin{array}{c}\text { One } \\
(4)\end{array}$ & $\begin{array}{c}\text { Two } \\
(5)\end{array}$ & $\begin{array}{c}\text { Three } \\
(6)\end{array}$ & $\begin{array}{c}\text { Four } \\
(7)\end{array}$ & $\begin{array}{c}\text { One } \\
(8)\end{array}$ & $\begin{array}{l}\text { Two } \\
(9)\end{array}$ \\
\hline Rural & $\begin{array}{c}0.965 \\
{[0.202]}\end{array}$ & $\begin{array}{c}0.965 \\
{[0.201]}\end{array}$ & $\begin{array}{c}0.965 \\
{[0.202]}\end{array}$ & $\begin{array}{c}1.145 \\
{[0.173]}\end{array}$ & $\begin{array}{c}1.146 \\
{[0.171]}\end{array}$ & $\begin{array}{c}1.077 \\
{[0.244]}\end{array}$ & $\begin{array}{c}1.067 \\
{[0.243]}\end{array}$ & $\begin{array}{c}1.144 \\
{[0.171]}\end{array}$ & $\begin{array}{c}1.040 \\
{[0.295]}\end{array}$ \\
\hline NUTS1 Region 1 & $\begin{array}{c}0.756 \\
{[0.415]}\end{array}$ & $\begin{array}{c}0.777 \\
{[0.400]}\end{array}$ & $\begin{array}{c}0.771 \\
{[0.397]}\end{array}$ & $\begin{array}{c}0.628 \\
{[0.228]}\end{array}$ & $\begin{array}{c}0.618 \\
{[0.213]}\end{array}$ & $\begin{array}{c}0.663 \\
{[0.376]}\end{array}$ & $\begin{array}{c}0.662 \\
{[0.376]}\end{array}$ & $\begin{array}{c}0.621 \\
{[0.212]}\end{array}$ & $\begin{array}{c}0.633 \\
{[0.437]}\end{array}$ \\
\hline NUTS1 Region 2 & $\begin{array}{c}0.469 \\
{[0.272]}\end{array}$ & $\begin{array}{c}0.456 \\
{[0.273]}\end{array}$ & $\begin{array}{c}0.449 \\
{[0.271]}\end{array}$ & $\begin{array}{c}0.774 \\
{[0.358]}\end{array}$ & $\begin{array}{c}0.808 \\
{[0.354]}\end{array}$ & $\begin{array}{c}0.457 \\
{[0.300]}\end{array}$ & $\begin{array}{c}0.432 \\
{[0.285]}\end{array}$ & $\begin{array}{c}0.800 \\
{[0.351]}\end{array}$ & $\begin{array}{c}0.349 \\
{[0.300]}\end{array}$ \\
\hline NUTS1 Region 3 & $\begin{array}{l}0.468^{*} \\
{[0.187]}\end{array}$ & $\begin{array}{l}0.464 * \\
{[0.192]}\end{array}$ & $\begin{array}{l}0.463^{*} \\
{[0.192]}\end{array}$ & $\begin{array}{c}0.808 \\
{[0.239]}\end{array}$ & $\begin{array}{c}0.801 \\
{[0.241]}\end{array}$ & $\begin{array}{c}0.426^{*} \\
{[0.202]}\end{array}$ & $\begin{array}{l}0.415^{*} \\
{[0.207]}\end{array}$ & $\begin{array}{c}0.802 \\
{[0.242]}\end{array}$ & $\begin{array}{l}0.318^{*} \\
{[0.206]}\end{array}$ \\
\hline NUTS1 Region 4 & $\begin{array}{c}2.428 \\
{[1.602]}\end{array}$ & $\begin{array}{c}2.448 \\
{[1.563]}\end{array}$ & $\begin{array}{c}2.437 \\
{[1.567]}\end{array}$ & $\begin{array}{c}0.819 \\
{[0.323]}\end{array}$ & $\begin{array}{c}0.809 \\
{[0.333]}\end{array}$ & $\begin{array}{c}2.306 \\
{[1.623]}\end{array}$ & $\begin{array}{c}2.303 \\
{[1.621]}\end{array}$ & $\begin{array}{c}0.810 \\
{[0.335]}\end{array}$ & $\begin{array}{c}3.580 \\
{[3.150]}\end{array}$ \\
\hline NUTS1 Region 5 & $\begin{array}{c}1.398 \\
{[0.678]}\end{array}$ & $\begin{array}{c}1.392 \\
{[0.691]}\end{array}$ & $\begin{array}{c}1.386 \\
{[0.694]}\end{array}$ & $\begin{array}{c}0.790 \\
{[0.256]}\end{array}$ & $\begin{array}{c}0.790 \\
{[0.258]}\end{array}$ & $\begin{array}{c}1.421 \\
{[0.742]}\end{array}$ & $\begin{array}{c}1.419 \\
{[0.752]}\end{array}$ & $\begin{array}{c}0.789 \\
{[0.259]}\end{array}$ & $\begin{array}{c}1.906 \\
{[1.286]}\end{array}$ \\
\hline NUTS1 Region 6 & $\begin{array}{c}0.997 \\
{[0.375]}\end{array}$ & $\begin{array}{c}0.963 \\
{[0.360]}\end{array}$ & $\begin{array}{c}0.973 \\
{[0.368]}\end{array}$ & $\begin{array}{c}0.804 \\
{[0.182]}\end{array}$ & $\begin{array}{c}0.801 \\
{[0.183]}\end{array}$ & $\begin{array}{c}0.822 \\
{[0.318]}\end{array}$ & $\begin{array}{c}0.830 \\
{[0.314]}\end{array}$ & $\begin{array}{c}0.801 \\
{[0.184]}\end{array}$ & $\begin{array}{c}0.850 \\
{[0.405]}\end{array}$ \\
\hline NUTS1 Region 7 & $\begin{array}{c}1.206 \\
{[0.548]}\end{array}$ & $\begin{array}{c}1.206 \\
{[0.545]}\end{array}$ & $\begin{array}{c}1.203 \\
{[0.545]}\end{array}$ & $\begin{array}{c}1.299 \\
{[0.362]}\end{array}$ & $\begin{array}{c}1.293 \\
{[0.368]}\end{array}$ & $\begin{array}{c}1.197 \\
{[0.571]}\end{array}$ & $\begin{array}{c}1.193 \\
{[0.569]}\end{array}$ & $\begin{array}{c}1.299 \\
{[0.373]}\end{array}$ & $\begin{array}{c}1.068 \\
{[0.639]}\end{array}$ \\
\hline NUTS1 Region 8 & $\begin{array}{c}0.670 \\
{[0.330]}\end{array}$ & $\begin{array}{c}0.688 \\
{[0.332]}\end{array}$ & $\begin{array}{c}0.691 \\
{[0.327]}\end{array}$ & $\begin{array}{c}1.081 \\
{[0.333]}\end{array}$ & $\begin{array}{c}1.132 \\
{[0.330]}\end{array}$ & $\begin{array}{c}0.825 \\
{[0.421]}\end{array}$ & $\begin{array}{c}0.800 \\
{[0.416]}\end{array}$ & $\begin{array}{c}1.125 \\
{[0.323]}\end{array}$ & $\begin{array}{c}0.738 \\
{[0.463]}\end{array}$ \\
\hline NUTS1 Region 9 & $\begin{array}{c}1.205 \\
{[0.526]}\end{array}$ & $\begin{array}{c}1.183 \\
{[0.476]}\end{array}$ & $\begin{array}{c}1.166 \\
{[0.463]}\end{array}$ & $\begin{array}{l}1.650^{*} \\
{[0.466]}\end{array}$ & $\begin{array}{l}1.651^{*} \\
{[0.462]}\end{array}$ & $\begin{array}{c}1.445 \\
{[0.700]}\end{array}$ & $\begin{array}{c}1.493 \\
{[0.677]}\end{array}$ & $\begin{array}{l}1.652 * \\
{[0.456]}\end{array}$ & $\begin{array}{c}1.280 \\
{[0.689]}\end{array}$ \\
\hline NUTS1 Region 10 & $\begin{array}{c}0.724 \\
{[0.257]}\end{array}$ & $\begin{array}{c}0.719 \\
{[0.263]}\end{array}$ & $\begin{array}{c}0.721 \\
{[0.268]}\end{array}$ & $\begin{array}{c}0.830 \\
{[0.206]}\end{array}$ & $\begin{array}{c}0.830 \\
{[0.207]}\end{array}$ & $\begin{array}{c}0.796 \\
{[0.327]}\end{array}$ & $\begin{array}{c}0.806 \\
{[0.349]}\end{array}$ & $\begin{array}{c}0.833 \\
{[0.210]}\end{array}$ & $\begin{array}{c}0.787 \\
{[0.446]}\end{array}$ \\
\hline NUTS1 Region 11 & $\begin{array}{c}1.521 \\
{[0.612]}\end{array}$ & $\begin{array}{c}1.460 \\
{[0.545]}\end{array}$ & $\begin{array}{c}1.445 \\
{[0.538]}\end{array}$ & $\begin{array}{c}1.352 \\
{[0.379]}\end{array}$ & $\begin{array}{c}1.350 \\
{[0.380]}\end{array}$ & $\begin{array}{c}1.609 \\
{[0.715]}\end{array}$ & $\begin{array}{c}1.566 \\
{[0.673]}\end{array}$ & $\begin{array}{c}1.344 \\
{[0.376]}\end{array}$ & $\begin{array}{c}1.656 \\
{[0.869]}\end{array}$ \\
\hline NUTS1 Region 12 & $\begin{array}{c}1.075 \\
{[0.398]}\end{array}$ & $\begin{array}{c}1.082 \\
{[0.412]}\end{array}$ & $\begin{array}{c}1.086 \\
{[0.413]}\end{array}$ & $\begin{array}{c}0.969 \\
{[0.245]}\end{array}$ & $\begin{array}{c}0.954 \\
{[0.235]}\end{array}$ & $\begin{array}{c}0.945 \\
{[0.396]}\end{array}$ & $\begin{array}{c}0.941 \\
{[0.388]}\end{array}$ & $\begin{array}{c}0.954 \\
{[0.233]}\end{array}$ & $\begin{array}{c}1.019 \\
{[0.530]}\end{array}$ \\
\hline
\end{tabular}

Notes: The sample includes observations from the 2013 DHS. In panel (A), with 4-year intervals around the donut-hole there are 2,324 observations in all regressions. In panel (B), with 7-year intervals around the donut-hole, there are 4,061 observations when the dependent variable is rural and 4,063 observations in the other regressions. The policy dummy is one when month-year of birth is greater than 1987 January. Each cell comes from a separate logistic regresion of the specified outcome on the policy dummy and the specified time trends. Odds ratios and their standard errors are given. Standard errors are clustered at the month-year of birth level. Statistical significance is *** at 1 percent level, ${ }^{* *}$ at 5 percent level, * at 10 percent level. 
Table A4: Check of Continuity in the Relationships between the Outcome Variables and the

\section{Running Variable I - Cut-off at January 1978, Odds ratios}

\begin{tabular}{|c|c|c|c|c|c|c|c|c|c|c|c|c|}
\hline \multirow{4}{*}{$\begin{array}{l}\text { Degree of } \\
\text { Polynomial } \\
\end{array}$} & \multicolumn{6}{|c|}{ I) Levels } & \multicolumn{6}{|c|}{ II) Timing } \\
\hline & \multicolumn{4}{|c|}{ Single Time Trend } & \multicolumn{2}{|c|}{$\begin{array}{l}\text { Split Time } \\
\text { Trends } \\
\end{array}$} & \multicolumn{4}{|c|}{ Single Time Trend } & \multicolumn{2}{|c|}{$\begin{array}{l}\text { Split Time } \\
\text { Trends } \\
\end{array}$} \\
\hline & $\begin{array}{l}\text { One } \\
(1)\end{array}$ & $\begin{array}{c}\text { Two } \\
(2)\end{array}$ & $\begin{array}{c}\text { Three } \\
(3)\end{array}$ & $\begin{array}{c}\text { Four } \\
(4)\end{array}$ & $\begin{array}{l}\text { One } \\
(5)\end{array}$ & $\begin{array}{c}\text { Two } \\
(6)\end{array}$ & $\begin{array}{l}\text { One } \\
(7)\end{array}$ & $\begin{array}{c}\text { Two } \\
(8)\end{array}$ & $\begin{array}{c}\text { Three } \\
(9)\end{array}$ & $\begin{array}{l}\text { Four } \\
(10) \\
\end{array}$ & $\begin{array}{l}\text { One } \\
(11)\end{array}$ & $\begin{array}{l}\text { Two } \\
(12) \\
\end{array}$ \\
\hline & \multicolumn{6}{|c|}{ I-A) Dependent Variable: Ever Married } & \multicolumn{6}{|c|}{$\begin{array}{l}\text { II-A) Dependent Variable: Marriage status } \\
\text { conditional on not being married until that age }\end{array}$} \\
\hline Age $=13$ & $\begin{array}{c}0.419 \\
{[0.281]}\end{array}$ & $\begin{array}{c}0.488 \\
{[0.319]}\end{array}$ & $\begin{array}{c}0.295 \\
{[0.273]}\end{array}$ & $\begin{array}{c}0.301 \\
{[0.285]}\end{array}$ & $\begin{array}{c}0.486 \\
{[0.314]}\end{array}$ & $\begin{array}{c}0.234 \\
{[0.276]}\end{array}$ & -- & -- & -- & -- & -- & -- \\
\hline Age $=14$ & $\begin{array}{c}0.812 \\
{[0.354]}\end{array}$ & $\begin{array}{c}0.862 \\
{[0.349]}\end{array}$ & $\begin{array}{c}0.653 \\
{[0.430]}\end{array}$ & $\begin{array}{c}0.699 \\
{[0.434]}\end{array}$ & $\begin{array}{c}0.858 \\
{[0.335]}\end{array}$ & $\begin{array}{c}0.613 \\
{[0.469]}\end{array}$ & $\begin{array}{c}1.345 \\
(0.854)\end{array}$ & $\begin{array}{c}1.309 \\
(0.761)\end{array}$ & $\begin{array}{c}1.191 \\
(1.124)\end{array}$ & $\begin{array}{c}1.169 \\
(0.987)\end{array}$ & $\begin{array}{c}1.280 \\
(0.712)\end{array}$ & $\begin{array}{c}0.987 \\
(0.996)\end{array}$ \\
\hline Age $=15$ & $\begin{array}{c}0.953 \\
{[0.286]}\end{array}$ & $\begin{array}{c}0.957 \\
{[0.277]}\end{array}$ & $\begin{array}{c}0.980 \\
{[0.511]}\end{array}$ & $\begin{array}{c}0.979 \\
{[0.515]}\end{array}$ & $\begin{array}{c}0.953 \\
{[0.273]}\end{array}$ & $\begin{array}{c}0.956 \\
{[0.630]}\end{array}$ & $\begin{array}{c}1.093 \\
(0.400)\end{array}$ & $\begin{array}{c}1.096 \\
(0.405)\end{array}$ & $\begin{array}{c}1.437 \\
(0.962)\end{array}$ & $\begin{array}{c}1.511 \\
(1.108)\end{array}$ & $\begin{array}{c}1.100 \\
(0.412)\end{array}$ & $\begin{array}{c}1.634 \\
(1.545)\end{array}$ \\
\hline Age $=16$ & $\begin{array}{c}0.982 \\
{[0.229]}\end{array}$ & $\begin{array}{c}0.984 \\
{[0.229]}\end{array}$ & $\begin{array}{c}1.030 \\
{[0.408]}\end{array}$ & $\begin{array}{c}1.018 \\
{[0.411]}\end{array}$ & $\begin{array}{c}0.982 \\
{[0.229]}\end{array}$ & $\begin{array}{c}1.010 \\
{[0.531]}\end{array}$ & $\begin{array}{c}1.012 \\
(0.307)\end{array}$ & $\begin{array}{c}0.990 \\
(0.302)\end{array}$ & $\begin{array}{c}1.097 \\
(0.543)\end{array}$ & $\begin{array}{c}1.046 \\
(0.539)\end{array}$ & $\begin{array}{c}0.986 \\
(0.305)\end{array}$ & $\begin{array}{c}1.044 \\
(0.719)\end{array}$ \\
\hline Age $=17$ & $\begin{array}{c}1.125 \\
{[0.232]}\end{array}$ & $\begin{array}{c}1.132 \\
{[0.232]}\end{array}$ & $\begin{array}{c}0.828 \\
{[0.297]}\end{array}$ & $\begin{array}{c}0.813 \\
{[0.298]}\end{array}$ & $\begin{array}{c}1.127 \\
{[0.231]}\end{array}$ & $\begin{array}{c}0.736 \\
{[0.349]}\end{array}$ & $\begin{array}{c}1.371 \\
(0.390)\end{array}$ & $\begin{array}{c}1.409 \\
(0.384)\end{array}$ & $\begin{array}{c}0.588 \\
(0.280)\end{array}$ & $\begin{array}{c}0.547 \\
(0.274)\end{array}$ & $\begin{array}{c}1.387 \\
(0.377)\end{array}$ & $\begin{array}{c}0.427 \\
(0.271)\end{array}$ \\
\hline Age $=18$ & $\begin{array}{c}1.028 \\
{[0.189]}\end{array}$ & $\begin{array}{c}1.028 \\
{[0.189]}\end{array}$ & $\begin{array}{c}0.973 \\
{[0.306]}\end{array}$ & $\begin{array}{c}0.971 \\
{[0.306]}\end{array}$ & $\begin{array}{c}1.026 \\
{[0.188]}\end{array}$ & $\begin{array}{c}0.955 \\
{[0.386]}\end{array}$ & $\begin{array}{c}0.856 \\
(0.217)\end{array}$ & $\begin{array}{c}0.853 \\
(0.212)\end{array}$ & $\begin{array}{c}1.335 \\
(0.570)\end{array}$ & $\begin{array}{c}1.328 \\
(0.559)\end{array}$ & $\begin{array}{c}0.852 \\
(0.211)\end{array}$ & $\begin{array}{c}1.522 \\
(0.804)\end{array}$ \\
\hline \multirow[t]{2}{*}{ Age $=19$} & $\begin{array}{c}0.938 \\
{[0.158]} \\
\end{array}$ & $\begin{array}{c}0.933 \\
{[0.156]} \\
\end{array}$ & $\begin{array}{c}0.793 \\
{[0.220]}\end{array}$ & $\begin{array}{c}0.794 \\
{[0.221]}\end{array}$ & $\begin{array}{c}0.930 \\
{[0.155]}\end{array}$ & $\begin{array}{c}0.781 \\
{[0.278]}\end{array}$ & $\begin{array}{c}0.773 \\
(0.229) \\
\end{array}$ & $\begin{array}{c}0.765 \\
(0.218) \\
\end{array}$ & $\begin{array}{c}0.542 \\
(0.265) \\
\end{array}$ & $\begin{array}{c}0.541 \\
(0.258) \\
\end{array}$ & $\begin{array}{c}0.763 \\
(0.213) \\
\end{array}$ & $\begin{array}{c}0.539 \\
(0.314) \\
\end{array}$ \\
\hline & \multicolumn{6}{|c|}{ I-B) Dependent Variable: Ever Given Birth } & \multicolumn{6}{|c|}{$\begin{array}{l}\text { II-B) Dependent Variable: Birth status conditional } \\
\text { on not giving birth until that age }\end{array}$} \\
\hline Age $=15$ & $\begin{array}{c}0.841 \\
{[0.494]}\end{array}$ & $\begin{array}{c}0.887 \\
{[0.480]}\end{array}$ & $\begin{array}{c}0.304 \\
{[0.282]}\end{array}$ & $\begin{array}{c}0.357 \\
{[0.302]}\end{array}$ & $\begin{array}{c}0.889 \\
{[0.460]}\end{array}$ & $\begin{array}{c}0.204 \\
{[0.206]}\end{array}$ & $\begin{array}{c}1.070 \\
(0.840)\end{array}$ & $\begin{array}{c}1.068 \\
(0.840)\end{array}$ & $\begin{array}{c}0.165 \\
(0.228)\end{array}$ & $\begin{array}{c}0.210 \\
(0.257)\end{array}$ & $\begin{array}{c}1.077 \\
(0.818)\end{array}$ & $\begin{array}{c}0.098 \\
(0.142)\end{array}$ \\
\hline Age $=16$ & $\begin{array}{c}0.853 \\
{[0.300]}\end{array}$ & $\begin{array}{c}0.883 \\
{[0.289]}\end{array}$ & $\begin{array}{c}0.783 \\
{[0.466]}\end{array}$ & $\begin{array}{c}0.784 \\
{[0.468]}\end{array}$ & $\begin{array}{c}0.876 \\
{[0.280]}\end{array}$ & $\begin{array}{c}0.696 \\
{[0.521]}\end{array}$ & $\begin{array}{c}0.863 \\
(0.363)\end{array}$ & $\begin{array}{c}0.882 \\
(0.346)\end{array}$ & $\begin{array}{c}1.573 \\
(1.060)\end{array}$ & $\begin{array}{c}1.598 \\
(1.156)\end{array}$ & $\begin{array}{c}0.874 \\
(0.341)\end{array}$ & $\begin{array}{c}2.161 \\
(2.001)\end{array}$ \\
\hline Age $=17$ & $\begin{array}{c}0.633^{*} \\
{[0.163]}\end{array}$ & $\begin{array}{c}0.657 * \\
{[0.162]}\end{array}$ & $\begin{array}{c}0.780 \\
{[0.344]}\end{array}$ & $\begin{array}{c}0.750 \\
{[0.342]}\end{array}$ & $\begin{array}{c}0.653 * \\
{[0.161]}\end{array}$ & $\begin{array}{c}0.739 \\
{[0.438]}\end{array}$ & $\begin{array}{c}0.485^{* *} \\
(0.164)\end{array}$ & $\begin{array}{c}0.499 * * \\
(0.163)\end{array}$ & $\begin{array}{c}0.764 \\
(0.443)\end{array}$ & $\begin{array}{c}0.682 \\
(0.416)\end{array}$ & $\begin{array}{c}0.490 * * \\
(0.161)\end{array}$ & $\begin{array}{c}0.718 \\
(0.576)\end{array}$ \\
\hline Age $=18$ & $\begin{array}{c}0.871 \\
{[0.199]}\end{array}$ & $\begin{array}{c}0.881 \\
{[0.197]}\end{array}$ & $\begin{array}{c}0.834 \\
{[0.333]}\end{array}$ & $\begin{array}{c}0.798 \\
{[0.326]}\end{array}$ & $\begin{array}{c}0.876 \\
{[0.197]}\end{array}$ & $\begin{array}{c}0.782 \\
{[0.417]}\end{array}$ & $\begin{array}{c}1.398 \\
(0.454)\end{array}$ & $\begin{array}{c}1.396 \\
(0.454)\end{array}$ & $\begin{array}{c}1.003 \\
(0.530)\end{array}$ & $\begin{array}{c}0.956 \\
(0.536)\end{array}$ & $\begin{array}{c}1.401 \\
(0.465)\end{array}$ & $\begin{array}{c}0.974 \\
(0.699)\end{array}$ \\
\hline Age $=19$ & $\begin{array}{c}0.954 \\
{[0.181]}\end{array}$ & $\begin{array}{c}0.958 \\
{[0.179]}\end{array}$ & $\begin{array}{c}0.836 \\
{[0.282]}\end{array}$ & $\begin{array}{c}0.831 \\
{[0.283]}\end{array}$ & $\begin{array}{c}0.955 \\
{[0.178]}\end{array}$ & $\begin{array}{c}0.778 \\
{[0.345]}\end{array}$ & $\begin{array}{c}1.109 \\
(0.329) \\
\end{array}$ & $\begin{array}{c}1.108 \\
(0.319) \\
\end{array}$ & $\begin{array}{c}0.876 \\
(0.421) \\
\end{array}$ & $\begin{array}{c}0.885 \\
(0.396) \\
\end{array}$ & $\begin{array}{c}1.102 \\
(0.311) \\
\end{array}$ & $\begin{array}{c}0.770 \\
(0.422)\end{array}$ \\
\hline
\end{tabular}

Notes: The data come from the 2013 DHS. The data are restricted to cohorts born before 1985 (i.e. those that are not affected by the 1997 education reform). In this range of birth cohorts, we take the 7-year interval with a donut-hole (as in the actual analysis) right before 1985. Hence, the sample includes the 1976 to 1985 birth cohorts, excluding the 1980 and 1981 birth cohorts. The counterfactual cut-off value of month-year of birth for the policy variable is January 1981. The number of observations is 4,013 in panels (I-A) and (I-B), 20,638 in panel (II-A), and 18,552 in panel (II-B). Each cell comes from a separate logistic regression of ever-married status in panel (I-A) and of ever-given-birth status in panel (I-B) at the specified age on the policy variable and the specified time trends in month and year of birth. The estimates in each column come from a separate logistic regression of marriage hazard status in panel (II-A) and of birth hazard status in panel (II-B) on the interactions of the policy variable with each age dummy and the interactions of the specified time trends in month and year of birth with each age dummy. Odds ratios and their standard errors are given as estimated parameters. The standard errors are clustered at the month-and-year-of-birth level. Statistical significance is $* * *$ at 1 percent level, ** at 5 percent level, * at 10 percent level. 
Table A5: Check of Continuity in the Relationships between the Outcome Variables and the

\section{Running Variable II - Cut-off at January 1976, Odds Ratios}

\begin{tabular}{|c|c|c|c|c|c|c|c|c|c|c|c|c|}
\hline \multirow[b]{3}{*}{$\begin{array}{l}\text { Degree of } \\
\text { Polynomial } \\
\end{array}$} & \multicolumn{6}{|c|}{ I) Levels } & \multicolumn{6}{|c|}{ II) Timing } \\
\hline & \multicolumn{4}{|c|}{ Single Time Trend } & \multicolumn{2}{|c|}{$\begin{array}{l}\text { Split Time } \\
\text { Trends }\end{array}$} & \multicolumn{4}{|c|}{ Single Time Trend } & \multicolumn{2}{|c|}{ Split Time Trends } \\
\hline & $\begin{array}{l}\text { One } \\
(1)\end{array}$ & $\begin{array}{c}\text { Two } \\
(2)\end{array}$ & $\begin{array}{l}\text { Three } \\
(3)\end{array}$ & $\begin{array}{c}\text { Four } \\
(4)\end{array}$ & $\begin{array}{l}\text { One } \\
(5)\end{array}$ & $\begin{array}{l}\text { Two } \\
(6) \\
\end{array}$ & $\begin{array}{l}\text { One } \\
(7)\end{array}$ & $\begin{array}{c}\text { Two } \\
(8)\end{array}$ & $\begin{array}{l}\text { Three } \\
(9)\end{array}$ & $\begin{array}{l}\text { Four } \\
(10) \\
\end{array}$ & $\begin{array}{l}\text { One } \\
(11)\end{array}$ & $\begin{array}{l}\text { Two } \\
(12) \\
\end{array}$ \\
\hline & \multicolumn{6}{|c|}{ I-A) Dependent Variable: Ever Married } & \multicolumn{6}{|c|}{$\begin{array}{l}\text { II-A) Dependent Variable: Marriage status } \\
\text { conditional on not being married until that age }\end{array}$} \\
\hline Age $=13$ & $\begin{array}{c}0.585 \\
{[0.386]}\end{array}$ & $\begin{array}{c}0.541 \\
{[0.362]}\end{array}$ & $\begin{array}{l}0.144 * \\
{[0.155]}\end{array}$ & $\begin{array}{l}0.151^{*} \\
{[0.165]}\end{array}$ & $\begin{array}{c}0.549 \\
{[0.367]}\end{array}$ & $\begin{array}{c}0.093 * \\
{[0.128]}\end{array}$ & -- & -- & -- & -- & -- & $\begin{array}{l}-- \\
--\end{array}$ \\
\hline Age $=14$ & $\begin{array}{c}1.071 \\
{[0.414]}\end{array}$ & $\begin{array}{c}1.066 \\
{[0.408]}\end{array}$ & $\begin{array}{c}0.564 \\
{[0.345]}\end{array}$ & $\begin{array}{c}0.558 \\
{[0.366]}\end{array}$ & $\begin{array}{c}1.070 \\
{[0.413]}\end{array}$ & $\begin{array}{c}0.444 \\
{[0.389]}\end{array}$ & $\begin{array}{c}1.698 \\
(0.800)\end{array}$ & $\begin{array}{c}1.526 \\
(0.672)\end{array}$ & $\begin{array}{c}1.384 \\
(0.996)\end{array}$ & $\begin{array}{c}2.175 \\
(1.892)\end{array}$ & $\begin{array}{c}1.591 \\
(0.719)\end{array}$ & $\begin{array}{c}2.774 \\
(3.284)\end{array}$ \\
\hline Age $=15$ & $\begin{array}{c}1.231 \\
{[0.350]}\end{array}$ & $\begin{array}{c}1.223 \\
{[0.346]}\end{array}$ & $\begin{array}{c}0.808 \\
{[0.388]}\end{array}$ & $\begin{array}{c}0.815 \\
{[0.399]}\end{array}$ & $\begin{array}{c}1.225 \\
{[0.347]}\end{array}$ & $\begin{array}{c}0.770 \\
{[0.489]}\end{array}$ & $\begin{array}{c}1.360 \\
(0.507)\end{array}$ & $\begin{array}{c}1.352 \\
(0.502)\end{array}$ & $\begin{array}{c}1.084 \\
(0.678)\end{array}$ & $\begin{array}{c}1.078 \\
(0.668)\end{array}$ & $\begin{array}{c}1.349 \\
(0.499)\end{array}$ & $\begin{array}{c}1.146 \\
(0.908)\end{array}$ \\
\hline Age $=16$ & $\begin{array}{c}0.940 \\
{[0.217]}\end{array}$ & $\begin{array}{c}0.939 \\
{[0.215]}\end{array}$ & $\begin{array}{c}0.852 \\
{[0.335]}\end{array}$ & $\begin{array}{c}0.851 \\
{[0.334]}\end{array}$ & $\begin{array}{c}0.938 \\
{[0.215]}\end{array}$ & $\begin{array}{c}0.862 \\
{[0.424]}\end{array}$ & $\begin{array}{c}0.712 \\
(0.191)\end{array}$ & $\begin{array}{c}0.713 \\
(0.191)\end{array}$ & $\begin{array}{c}0.909 \\
(0.409)\end{array}$ & $\begin{array}{c}0.928 \\
(0.398)\end{array}$ & $\begin{array}{c}0.716 \\
(0.189)\end{array}$ & $\begin{array}{c}1.035 \\
(0.542)\end{array}$ \\
\hline Age $=17$ & $\begin{array}{c}1.047 \\
{[0.198]}\end{array}$ & $\begin{array}{c}1.041 \\
{[0.196]}\end{array}$ & $\begin{array}{c}1.253 \\
{[0.382]}\end{array}$ & $\begin{array}{c}1.246 \\
{[0.382]}\end{array}$ & $\begin{array}{c}1.039 \\
{[0.196]}\end{array}$ & $\begin{array}{c}1.346 \\
{[0.513]}\end{array}$ & $\begin{array}{c}1.227 \\
(0.324)\end{array}$ & $\begin{array}{c}1.209 \\
(0.312)\end{array}$ & $\begin{array}{l}2.162 * \\
(0.865)\end{array}$ & $\begin{array}{l}2.146^{*} \\
(0.861)\end{array}$ & $\begin{array}{c}1.204 \\
(0.309)\end{array}$ & $\begin{array}{l}2.537 * \\
(1.279)\end{array}$ \\
\hline Age $=18$ & $\begin{array}{c}1.086 \\
{[0.189]}\end{array}$ & $\begin{array}{c}1.073 \\
{[0.186]}\end{array}$ & $\begin{array}{c}1.134 \\
{[0.312]}\end{array}$ & $\begin{array}{c}1.118 \\
{[0.311]}\end{array}$ & $\begin{array}{c}1.068 \\
{[0.186]}\end{array}$ & $\begin{array}{c}1.180 \\
{[0.407]}\end{array}$ & $\begin{array}{c}1.141 \\
(0.271)\end{array}$ & $\begin{array}{c}1.124 \\
(0.261)\end{array}$ & $\begin{array}{c}0.899 \\
(0.304)\end{array}$ & $\begin{array}{c}0.886 \\
(0.295)\end{array}$ & $\begin{array}{c}1.117 \\
(0.258)\end{array}$ & $\begin{array}{c}0.877 \\
(0.362)\end{array}$ \\
\hline \multirow[t]{2}{*}{ Age $=19$} & $\begin{array}{c}1.048 \\
{[0.187]}\end{array}$ & $\begin{array}{r}1.045 \\
{[0.190]} \\
\end{array}$ & $\begin{array}{c}1.170 \\
{[0.337]} \\
\end{array}$ & $\begin{array}{c}1.128 \\
{[0.332]} \\
\end{array}$ & $\begin{array}{c}1.041 \\
{[0.191]} \\
\end{array}$ & $\begin{array}{c}1.146 \\
{[0.417]} \\
\end{array}$ & $\begin{array}{c}0.945 \\
(0.294) \\
\end{array}$ & $\begin{array}{c}0.976 \\
(0.314) \\
\end{array}$ & $\begin{array}{r}1.178 \\
(0.590) \\
\end{array}$ & $\begin{array}{c}1.097 \\
(0.529) \\
\end{array}$ & $\begin{array}{c}0.970 \\
(0.314) \\
\end{array}$ & $\begin{array}{r}1.005 \\
(0.591) \\
\end{array}$ \\
\hline & \multicolumn{6}{|c|}{ I-B) Dependent Variable: Ever Given Birth } & \multicolumn{6}{|c|}{$\begin{array}{l}\text { II-B) Dependent Variable: Birth status conditional on } \\
\text { not giving birth until that age }\end{array}$} \\
\hline Age $=15$ & $\begin{array}{l}1.311 \\
{[0.595]}\end{array}$ & $\begin{array}{c}1.311 \\
{[0.595]}\end{array}$ & $\begin{array}{c}0.724 \\
{[0.518]}\end{array}$ & $\begin{array}{c}0.768 \\
{[0.642]}\end{array}$ & $\begin{array}{c}1.350 \\
{[0.636]}\end{array}$ & $\begin{array}{c}0.722 \\
{[0.842]}\end{array}$ & $\begin{array}{l}1.511 \\
(0.872)\end{array}$ & $\begin{array}{c}1.520 \\
(0.874)\end{array}$ & $\begin{array}{c}1.669 \\
(1.567)\end{array}$ & $\begin{array}{c}3.013 \\
(3.782)\end{array}$ & $\begin{array}{c}1.605 \\
(0.972)\end{array}$ & $\begin{array}{c}6.599 \\
(12.045)\end{array}$ \\
\hline Age $=16$ & $\begin{array}{c}1.047 \\
{[0.308]}\end{array}$ & $\begin{array}{c}1.041 \\
{[0.303]}\end{array}$ & $\begin{array}{c}0.619 \\
{[0.280]}\end{array}$ & $\begin{array}{c}0.620 \\
{[0.288]}\end{array}$ & $\begin{array}{c}1.043 \\
{[0.305]}\end{array}$ & $\begin{array}{c}0.523 \\
{[0.320]}\end{array}$ & $\begin{array}{c}0.907 \\
(0.367)\end{array}$ & $\begin{array}{c}0.904 \\
(0.357)\end{array}$ & $\begin{array}{c}0.575 \\
(0.351)\end{array}$ & $\begin{array}{c}0.578 \\
(0.338)\end{array}$ & $\begin{array}{c}0.900 \\
(0.351)\end{array}$ & $\begin{array}{c}0.489 \\
(0.358)\end{array}$ \\
\hline Age $=17$ & $\begin{array}{c}0.824 \\
{[0.203]}\end{array}$ & $\begin{array}{c}0.824 \\
{[0.198]}\end{array}$ & $\begin{array}{c}0.592 \\
{[0.231]}\end{array}$ & $\begin{array}{c}0.593 \\
{[0.230]}\end{array}$ & $\begin{array}{c}0.821 \\
{[0.196]}\end{array}$ & $\begin{array}{c}0.548 \\
{[0.268]}\end{array}$ & $\begin{array}{c}0.668 \\
(0.248)\end{array}$ & $\begin{array}{c}0.683 \\
(0.242)\end{array}$ & $\begin{array}{c}0.599 \\
(0.351)\end{array}$ & $\begin{array}{c}0.623 \\
(0.340)\end{array}$ & $\begin{array}{c}0.684 \\
(0.236)\end{array}$ & $\begin{array}{c}0.636 \\
(0.430)\end{array}$ \\
\hline Age $=18$ & $\begin{array}{c}0.913 \\
{[0.188]}\end{array}$ & $\begin{array}{c}0.911 \\
{[0.186]}\end{array}$ & $\begin{array}{c}0.881 \\
{[0.293]}\end{array}$ & $\begin{array}{c}0.874 \\
{[0.289]}\end{array}$ & $\begin{array}{c}0.909 \\
{[0.186]}\end{array}$ & $\begin{array}{c}0.889 \\
{[0.366]}\end{array}$ & $\begin{array}{c}1.057 \\
(0.300)\end{array}$ & $\begin{array}{c}1.064 \\
(0.308)\end{array}$ & $\begin{array}{c}1.557 \\
(0.737)\end{array}$ & $\begin{array}{c}1.521 \\
(0.709)\end{array}$ & $\begin{array}{c}1.067 \\
(0.310)\end{array}$ & $\begin{array}{c}1.803 \\
(1.061)\end{array}$ \\
\hline Age $=19$ & $\begin{array}{c}1.120 \\
{[0.191]}\end{array}$ & $\begin{array}{c}1.110 \\
{[0.189]}\end{array}$ & $\begin{array}{c}1.012 \\
{[0.275]}\end{array}$ & $\begin{array}{c}0.997 \\
{[0.274]}\end{array}$ & $\begin{array}{c}1.106 \\
{[0.189]}\end{array}$ & $\begin{array}{c}0.984 \\
{[0.335]}\end{array}$ & $\begin{array}{c}1.535 \\
(0.409) \\
\end{array}$ & $\begin{array}{c}1.501 \\
(0.392) \\
\end{array}$ & $\begin{array}{c}1.247 \\
(0.544) \\
\end{array}$ & $\begin{array}{c}1.228 \\
(0.523) \\
\end{array}$ & $\begin{array}{c}1.489 \\
(0.385) \\
\end{array}$ & $\begin{array}{c}1.137 \\
(0.591) \\
\end{array}$ \\
\hline
\end{tabular}

Notes: The data come from the 2013 DHS. The data are restricted to cohorts born before 1985 (i.e. those that are not affected by the 1997 education reform). In this range of birth cohorts, we take the 1968 to 1983 birth cohorts excluding the 1975 and 1976 birth cohorts (7-year intervals around the donut-hole). The counterfactual cut-off value of month-year of birth for the policy variable is January 1983. The number of observations is 3,994 in panels (I-A) and (I-B), 20,354 in panel (II-A), and 18,395 in panel (II-B). Each cell comes from a separate logistic regression of ever-married status in panel (I-A) and of ever-given-birth status in panel (I-B) at the specified age on the policy variable and the specified time trends in month and year of birth. The estimates in each column come from a separate logistic regression of marriage hazard status in panel (II-A) and of birth hazard status in panel (II-B) on the interactions of the policy variable with each age dummy and the interactions of the specified time trends in month and year of birth with each age dummy. Odds ratios and their standard errors are given as estimated parameters. The standard errors are clustered at the month-and-year-ofbirth level. Statistical significance is *** at 1 percent level, ** at 5 percent level, * at 10 percent level. 
Table A6: Check of Continuity in the Relationships between the Outcome Variables and the

\section{Running Variable III - Cut-off at January 1974, Odds ratios}

\begin{tabular}{|c|c|c|c|c|c|c|c|c|c|c|c|c|}
\hline \multirow[b]{3}{*}{$\begin{array}{l}\text { Degree of } \\
\text { Polynomial }\end{array}$} & \multicolumn{6}{|c|}{ I) Levels } & \multicolumn{6}{|c|}{ II) Timing } \\
\hline & \multicolumn{4}{|c|}{ Single Time Trend } & \multicolumn{2}{|c|}{ Split Time Trends } & \multicolumn{4}{|c|}{ Single Time Trend } & \multicolumn{2}{|c|}{$\begin{array}{l}\text { Split Time } \\
\text { Trends }\end{array}$} \\
\hline & $\begin{array}{c}\text { One } \\
(1)\end{array}$ & $\begin{array}{c}\text { Two } \\
(2)\end{array}$ & $\begin{array}{l}\text { Three } \\
(3)\end{array}$ & $\begin{array}{c}\text { Four } \\
(4)\end{array}$ & $\begin{array}{l}\text { One } \\
(5)\end{array}$ & $\begin{array}{l}\text { Two } \\
(6)\end{array}$ & $\begin{array}{l}\text { One } \\
(7)\end{array}$ & $\begin{array}{c}\text { Two } \\
(8)\end{array}$ & $\begin{array}{l}\text { Three } \\
(9)\end{array}$ & $\begin{array}{r}\text { Four } \\
(10)\end{array}$ & $\begin{array}{l}\text { One } \\
(11)\end{array}$ & $\begin{array}{l}\text { Two } \\
(12) \\
\end{array}$ \\
\hline & \multicolumn{6}{|c|}{ I-A) Dependent Variable: Ever Married } & \multicolumn{6}{|c|}{$\begin{array}{l}\text { II-A) Dependent Variable: Marriage status } \\
\text { conditional on not being married until that age }\end{array}$} \\
\hline Age $=13$ & $\begin{array}{c}2.544 \\
{[1.499]}\end{array}$ & $\begin{array}{c}2.560 \\
{[1.530]}\end{array}$ & $\begin{array}{l}12.131 * * \\
{[12.153]}\end{array}$ & $\begin{array}{l}12.057 * * \\
{[12.381]}\end{array}$ & $\begin{array}{c}2.568 \\
{[1.510]}\end{array}$ & $\begin{array}{l}17.943 * * \\
{[22.587]}\end{array}$ & -- & $\begin{array}{l}-- \\
--\end{array}$ & -- & $\begin{array}{l}-- \\
--\end{array}$ & $\begin{array}{l}-- \\
--\end{array}$ & -- \\
\hline Age $=14$ & $\begin{array}{c}1.718 \\
{[0.608]}\end{array}$ & $\begin{array}{c}1.752 \\
{[0.626]}\end{array}$ & $\begin{array}{c}3.665^{* *} \\
{[2.078]}\end{array}$ & $\begin{array}{c}3.607 * * \\
{[2.049]}\end{array}$ & $\begin{array}{c}1.743 \\
{[0.618]}\end{array}$ & $\begin{array}{c}4.513 * * \\
{[3.182]}\end{array}$ & $\begin{array}{c}1.272 \\
(0.635)\end{array}$ & $\begin{array}{c}1.258 \\
(0.613)\end{array}$ & $\begin{array}{c}1.510 \\
(1.185)\end{array}$ & $\begin{array}{c}1.439 \\
(1.075)\end{array}$ & $\begin{array}{c}1.251 \\
(0.607)\end{array}$ & $\begin{array}{c}1.611 \\
(1.513)\end{array}$ \\
\hline Age $=15$ & $\begin{array}{c}1.120 \\
{[0.286]}\end{array}$ & $\begin{array}{c}1.124 \\
{[0.285]}\end{array}$ & $\begin{array}{c}1.450 \\
{[0.602]}\end{array}$ & $\begin{array}{c}1.424 \\
{[0.587]}\end{array}$ & $\begin{array}{c}1.121 \\
{[0.285]}\end{array}$ & $\begin{array}{c}1.477 \\
{[0.758]}\end{array}$ & $\begin{array}{c}0.763 \\
(0.284)\end{array}$ & $\begin{array}{c}0.762 \\
(0.282)\end{array}$ & $\begin{array}{c}0.638 \\
(0.378)\end{array}$ & $\begin{array}{c}0.637 \\
(0.371)\end{array}$ & $\begin{array}{c}0.762 \\
(0.282)\end{array}$ & $\begin{array}{c}0.544 \\
(0.389)\end{array}$ \\
\hline Age $=16$ & $\begin{array}{c}0.792 \\
{[0.176]}\end{array}$ & $\begin{array}{c}0.790 \\
{[0.174]}\end{array}$ & $\begin{array}{c}0.876 \\
{[0.314]}\end{array}$ & $\begin{array}{c}0.874 \\
{[0.313]}\end{array}$ & $\begin{array}{c}0.789 \\
{[0.174]}\end{array}$ & $\begin{array}{c}0.846 \\
{[0.381]}\end{array}$ & $\begin{array}{c}0.541 * * \\
(0.161)\end{array}$ & $\begin{array}{c}0.552 * * \\
(0.158)\end{array}$ & $\begin{array}{c}0.510 \\
(0.230)\end{array}$ & $\begin{array}{c}0.507 \\
(0.226)\end{array}$ & $\begin{array}{c}0.552^{* *} \\
(0.157)\end{array}$ & $\begin{array}{c}0.442 \\
(0.248)\end{array}$ \\
\hline Age $=17$ & $\begin{array}{c}0.937 \\
{[0.184]}\end{array}$ & $\begin{array}{c}0.925 \\
{[0.181]}\end{array}$ & $\begin{array}{c}0.915 \\
{[0.298]}\end{array}$ & $\begin{array}{c}0.909 \\
{[0.297]}\end{array}$ & $\begin{array}{c}0.924 \\
{[0.181]}\end{array}$ & $\begin{array}{c}0.898 \\
{[0.376]}\end{array}$ & $\begin{array}{c}1.228 \\
(0.341)\end{array}$ & $\begin{array}{c}1.185 \\
(0.325)\end{array}$ & $\begin{array}{c}0.975 \\
(0.435)\end{array}$ & $\begin{array}{c}0.962 \\
(0.429)\end{array}$ & $\begin{array}{c}1.182 \\
(0.324)\end{array}$ & $\begin{array}{c}0.994 \\
(0.577)\end{array}$ \\
\hline Age $=18$ & $\begin{array}{c}0.993 \\
{[0.171]}\end{array}$ & $\begin{array}{c}0.989 \\
{[0.171]}\end{array}$ & $\begin{array}{c}0.884 \\
{[0.265]}\end{array}$ & $\begin{array}{c}0.874 \\
{[0.263]}\end{array}$ & $\begin{array}{c}0.987 \\
{[0.171]}\end{array}$ & $\begin{array}{c}0.774 \\
{[0.295]}\end{array}$ & $\begin{array}{c}1.104 \\
(0.293)\end{array}$ & $\begin{array}{c}1.121 \\
(0.303)\end{array}$ & $\begin{array}{c}0.861 \\
(0.426)\end{array}$ & $\begin{array}{c}0.853 \\
(0.415)\end{array}$ & $\begin{array}{c}1.122 \\
(0.306)\end{array}$ & $\begin{array}{c}0.636 \\
(0.382)\end{array}$ \\
\hline \multirow[t]{2}{*}{ Age $=19$} & $\begin{array}{c}1.173 \\
{[0.199]} \\
\end{array}$ & $\begin{array}{c}1.161 \\
{[0.198]} \\
\end{array}$ & $\begin{array}{c}1.029 \\
{[0.316]} \\
\end{array}$ & $\begin{array}{c}1.021 \\
{[0.318]}\end{array}$ & $\begin{array}{c}1.159 \\
{[0.199]} \\
\end{array}$ & $\begin{array}{c}0.927 \\
{[0.371]} \\
\end{array}$ & $\begin{array}{c}1.669 * \\
(0.455) \\
\end{array}$ & $\begin{array}{c}1.627^{*} \\
(0.436) \\
\end{array}$ & $\begin{array}{c}1.426 \\
(0.649) \\
\end{array}$ & $\begin{array}{c}1.461 \\
(0.669) \\
\end{array}$ & $\begin{array}{c}1.623 * \\
(0.435) \\
\end{array}$ & $\begin{array}{c}1.465 \\
(0.856) \\
\end{array}$ \\
\hline & \multicolumn{6}{|c|}{ I-B) Dependent Variable: Ever Given Birth } & \multicolumn{6}{|c|}{$\begin{array}{l}\text { II-B) Dependent Variable: Birth status conditional } \\
\text { on not giving birth until that age }\end{array}$} \\
\hline Age $=15$ & $\begin{array}{c}1.006 \\
{[0.525]}\end{array}$ & $\begin{array}{c}1.002 \\
{[0.518]}\end{array}$ & $\begin{array}{c}2.209 \\
{[1.816]}\end{array}$ & $\begin{array}{c}1.989 \\
{[1.542]}\end{array}$ & $\begin{array}{c}0.991 \\
{[0.507]}\end{array}$ & $\begin{array}{c}2.211 \\
{[2.089]}\end{array}$ & $\begin{array}{c}0.580 \\
(0.415)\end{array}$ & $\begin{array}{c}0.562 \\
(0.383)\end{array}$ & $\begin{array}{c}1.395 \\
(1.483)\end{array}$ & $\begin{array}{c}1.241 \\
(1.200)\end{array}$ & $\begin{array}{c}0.563 \\
(0.378)\end{array}$ & $\begin{array}{c}1.320 \\
(1.643)\end{array}$ \\
\hline Age $=16$ & $\begin{array}{c}1.026 \\
{[0.322]}\end{array}$ & $\begin{array}{c}1.026 \\
{[0.321]}\end{array}$ & $\begin{array}{c}1.549 \\
{[0.778]}\end{array}$ & $\begin{array}{c}1.520 \\
{[0.756]}\end{array}$ & $\begin{array}{c}1.022 \\
{[0.320]}\end{array}$ & $\begin{array}{c}1.550 \\
{[0.932]}\end{array}$ & $\begin{array}{c}1.042 \\
(0.458)\end{array}$ & $\begin{array}{c}1.043 \\
(0.466)\end{array}$ & $\begin{array}{c}1.159 \\
(0.861)\end{array}$ & $\begin{array}{c}1.178 \\
(0.903)\end{array}$ & $\begin{array}{c}1.044 \\
(0.469)\end{array}$ & $\begin{array}{c}1.124 \\
(1.044)\end{array}$ \\
\hline Age $=17$ & $\begin{array}{c}1.038 \\
{[0.277]}\end{array}$ & $\begin{array}{c}1.022 \\
{[0.266]}\end{array}$ & $\begin{array}{c}1.038 \\
{[0.460]}\end{array}$ & $\begin{array}{c}1.015 \\
{[0.438]}\end{array}$ & $\begin{array}{c}1.017 \\
{[0.262]}\end{array}$ & $\begin{array}{c}0.899 \\
{[0.478]}\end{array}$ & $\begin{array}{c}1.048 \\
(0.425)\end{array}$ & $\begin{array}{c}1.037 \\
(0.397)\end{array}$ & $\begin{array}{c}0.689 \\
(0.436)\end{array}$ & $\begin{array}{c}0.691 \\
(0.421)\end{array}$ & $\begin{array}{c}1.028 \\
(0.386)\end{array}$ & $\begin{array}{c}0.533 \\
(0.396)\end{array}$ \\
\hline Age $=18$ & $\begin{array}{c}0.869 \\
{[0.204]}\end{array}$ & $\begin{array}{c}0.860 \\
{[0.198]}\end{array}$ & $\begin{array}{c}0.788 \\
{[0.306]}\end{array}$ & $\begin{array}{c}0.784 \\
{[0.302]}\end{array}$ & $\begin{array}{c}0.857 \\
{[0.197]}\end{array}$ & $\begin{array}{c}0.696 \\
{[0.336]}\end{array}$ & $\begin{array}{c}0.684 \\
(0.245)\end{array}$ & $\begin{array}{c}0.685 \\
(0.240)\end{array}$ & $\begin{array}{c}0.542 \\
(0.323)\end{array}$ & $\begin{array}{c}0.540 \\
(0.328)\end{array}$ & $\begin{array}{c}0.685 \\
(0.239)\end{array}$ & $\begin{array}{c}0.483 \\
(0.367)\end{array}$ \\
\hline Age $=19$ & $\begin{array}{c}1.017 \\
{[0.174]}\end{array}$ & $\begin{array}{c}1.013 \\
{[0.174]}\end{array}$ & $\begin{array}{c}0.905 \\
{[0.263]}\end{array}$ & $\begin{array}{c}0.905 \\
{[0.265]}\end{array}$ & $\begin{array}{c}1.013 \\
{[0.174]} \\
\end{array}$ & $\begin{array}{c}0.795 \\
{[0.297]}\end{array}$ & $\begin{array}{c}1.296 \\
(0.359) \\
\end{array}$ & $\begin{array}{c}1.323 \\
(0.361) \\
\end{array}$ & $\begin{array}{c}1.185 \\
(0.551) \\
\end{array}$ & $\begin{array}{c}1.214 \\
(0.569) \\
\end{array}$ & $\begin{array}{c}1.337 \\
(0.365) \\
\end{array}$ & $\begin{array}{r}1.119 \\
(0.680) \\
\end{array}$ \\
\hline
\end{tabular}

Notes: The data come from the 2013 DHS. The data are restricted to cohorts born before 1985 (i.e. those that are not affected by the 1997 education reform). In this range of birth cohorts, we take the 1966 to 1981 birth cohorts excluding the 1973 and 1974 birth cohorts (7-year intervals around the donuthole). The counterfactual cut-off value of month-year of birth for the policy variable is January 1974. The number of observations is 3,792 in panels (I-A) and (I-B), 19,220 in panel (II-A), and 17,397 in panel (II-B). Each cell comes from a separate logistic regression of ever-married status in panel (I-A) and of ever-given-birth status in panel (I-B) at the specified age on the policy variable and the specified time trends in month and year of birth. The estimates in each column come from a separate logistic regression of marriage hazard status in panel (II-A) and of birth hazard status in panel (II-B) on the interactions of the policy variable with each age dummy and the interactions of the specified time trends in month and year of birth with each age dummy. Odds ratios and their standard errors are given as estimated parameters. The standard errors are clustered at the month-and-year-of-birth level. Statistical significance is $* * *$ at 1 percent level, $* *$ at 5 percent level, * at 10 percent level. 
Table A7: Check of Continuity in the Relationships between the Outcome Variables and the Running Variable IV - Cut-off at January 1972, Odds ratios

\begin{tabular}{|c|c|c|c|c|c|c|c|c|c|c|c|c|}
\hline \multirow[b]{3}{*}{$\begin{array}{l}\text { Degree of } \\
\text { Polynomial }\end{array}$} & \multicolumn{6}{|c|}{ I) Levels } & \multicolumn{6}{|c|}{ II) Timing } \\
\hline & \multicolumn{4}{|c|}{ Single Time Trend } & \multicolumn{2}{|c|}{$\begin{array}{l}\text { Split Time } \\
\text { Trends }\end{array}$} & \multicolumn{4}{|c|}{ Single Time Trend } & \multicolumn{2}{|c|}{$\begin{array}{l}\text { Split Time } \\
\text { Trends }\end{array}$} \\
\hline & $\begin{array}{l}\text { One } \\
(1)\end{array}$ & $\begin{array}{l}\text { Two } \\
(2) \\
\end{array}$ & $\begin{array}{c}\text { Three } \\
(3)\end{array}$ & $\begin{array}{c}\text { Four } \\
(4)\end{array}$ & $\begin{array}{l}\text { One } \\
(5)\end{array}$ & $\begin{array}{l}\text { Two } \\
(6)\end{array}$ & $\begin{array}{l}\text { One } \\
(7)\end{array}$ & $\begin{array}{c}\text { Two } \\
(8)\end{array}$ & $\begin{array}{l}\text { Three } \\
(9)\end{array}$ & $\begin{array}{l}\text { Four } \\
(10) \\
\end{array}$ & $\begin{array}{l}\text { One } \\
(11)\end{array}$ & $\begin{array}{l}\text { Two } \\
(12)\end{array}$ \\
\hline & \multicolumn{6}{|c|}{ I-A) Dependent Variable: Ever Married } & \multicolumn{6}{|c|}{$\begin{array}{l}\text { II-A) Dependent Variable: Marriage status } \\
\text { conditional on not being married until that age }\end{array}$} \\
\hline Age $=13$ & $\begin{array}{c}2.188 \\
{[1.279]}\end{array}$ & $\begin{array}{c}2.148 \\
{[1.182]}\end{array}$ & $\begin{array}{c}4.021 \\
{[3.435]}\end{array}$ & $\begin{array}{c}4.066 \\
{[3.556]}\end{array}$ & $\begin{array}{c}2.109 \\
{[1.152]}\end{array}$ & $\begin{array}{c}5.548 \\
{[6.665]}\end{array}$ & -- & $\begin{array}{l}-- \\
--\end{array}$ & $\begin{array}{l}-- \\
--\end{array}$ & $\begin{array}{l}-- \\
--\end{array}$ & $\begin{array}{l}-- \\
--\end{array}$ & $\begin{array}{l}-- \\
--\end{array}$ \\
\hline Age $=14$ & $\begin{array}{c}1.553 \\
{[0.577]}\end{array}$ & $\begin{array}{c}1.600 \\
{[0.622]}\end{array}$ & $\begin{array}{c}1.821 \\
{[1.122]}\end{array}$ & $\begin{array}{c}1.768 \\
{[1.069]}\end{array}$ & $\begin{array}{c}1.611 \\
{[0.636]}\end{array}$ & $\begin{array}{c}1.779 \\
{[1.379]}\end{array}$ & $\begin{array}{c}1.133 \\
(0.551)\end{array}$ & $\begin{array}{c}1.286 \\
(0.707)\end{array}$ & $\begin{array}{c}0.751 \\
(0.671)\end{array}$ & $\begin{array}{c}0.710 \\
(0.598)\end{array}$ & $\begin{array}{c}1.292 \\
(0.733)\end{array}$ & $\begin{array}{c}0.526 \\
(0.544)\end{array}$ \\
\hline Age $=15$ & $\begin{array}{c}1.425 \\
{[0.391]}\end{array}$ & $\begin{array}{c}1.495 \\
{[0.430]}\end{array}$ & $\begin{array}{c}1.375 \\
{[0.631]}\end{array}$ & $\begin{array}{c}1.342 \\
{[0.601]}\end{array}$ & $\begin{array}{c}1.513 \\
{[0.445]}\end{array}$ & $\begin{array}{c}1.350 \\
{[0.798]}\end{array}$ & $\begin{array}{c}1.293 \\
(0.470)\end{array}$ & $\begin{array}{c}1.366 \\
(0.536)\end{array}$ & $\begin{array}{c}1.024 \\
(0.626)\end{array}$ & $\begin{array}{c}1.009 \\
(0.602)\end{array}$ & $\begin{array}{c}1.388 \\
(0.558)\end{array}$ & $\begin{array}{c}1.015 \\
(0.780)\end{array}$ \\
\hline Age $=16$ & $\begin{array}{c}1.203 \\
{[0.303]}\end{array}$ & $\begin{array}{c}1.205 \\
{[0.310]}\end{array}$ & $\begin{array}{c}0.912 \\
{[0.372]}\end{array}$ & $\begin{array}{c}0.889 \\
{[0.349]}\end{array}$ & $\begin{array}{c}1.202 \\
{[0.310]}\end{array}$ & $\begin{array}{c}0.834 \\
{[0.418]}\end{array}$ & $\begin{array}{c}0.973 \\
(0.327)\end{array}$ & $\begin{array}{c}0.945 \\
(0.300)\end{array}$ & $\begin{array}{c}0.611 \\
(0.310)\end{array}$ & $\begin{array}{c}0.608 \\
(0.295)\end{array}$ & $\begin{array}{c}0.936 \\
(0.292)\end{array}$ & $\begin{array}{c}0.552 \\
(0.329)\end{array}$ \\
\hline Age $=17$ & $\begin{array}{c}1.206 \\
{[0.236]}\end{array}$ & $\begin{array}{c}1.210 \\
{[0.239]}\end{array}$ & $\begin{array}{c}0.788 \\
{[0.245]}\end{array}$ & $\begin{array}{c}0.775 \\
{[0.238]}\end{array}$ & $\begin{array}{c}1.207 \\
{[0.239]}\end{array}$ & $\begin{array}{c}0.690 \\
{[0.260]}\end{array}$ & $\begin{array}{c}1.175 \\
(0.308)\end{array}$ & $\begin{array}{c}1.178 \\
(0.316)\end{array}$ & $\begin{array}{c}0.646 \\
(0.286)\end{array}$ & $\begin{array}{c}0.660 \\
(0.300)\end{array}$ & $\begin{array}{c}1.180 \\
(0.317)\end{array}$ & $\begin{array}{c}0.547 \\
(0.303)\end{array}$ \\
\hline Age $=18$ & $\begin{array}{c}1.120 \\
{[0.208]}\end{array}$ & $\begin{array}{c}1.106 \\
{[0.202]}\end{array}$ & $\begin{array}{c}1.037 \\
{[0.289]}\end{array}$ & $\begin{array}{c}1.052 \\
{[0.295]}\end{array}$ & $\begin{array}{c}1.106 \\
{[0.202]}\end{array}$ & $\begin{array}{c}1.106 \\
{[0.381]}\end{array}$ & $\begin{array}{c}0.942 \\
(0.256)\end{array}$ & $\begin{array}{c}0.901 \\
(0.233)\end{array}$ & $\begin{array}{c}1.768 \\
(0.657)\end{array}$ & $\begin{array}{l}2.079 * \\
(0.812)\end{array}$ & $\begin{array}{c}0.907 \\
(0.234)\end{array}$ & $\begin{array}{c}2.999 * * \\
(1.462)\end{array}$ \\
\hline \multirow[t]{2}{*}{ Age $=19$} & $\begin{array}{c}1.041 \\
{[0.191]} \\
\end{array}$ & $\begin{array}{c}1.040 \\
{[0.190]} \\
\end{array}$ & $\begin{array}{c}0.971 \\
{[0.263]} \\
\end{array}$ & $\begin{array}{c}0.974 \\
{[0.263]} \\
\end{array}$ & $\begin{array}{c}1.042 \\
{[0.190]} \\
\end{array}$ & $\begin{array}{c}0.980 \\
{[0.322]}\end{array}$ & $\begin{array}{c}0.863 \\
(0.296) \\
\end{array}$ & $\begin{array}{c}0.889 \\
(0.310) \\
\end{array}$ & $\begin{array}{c}0.821 \\
(0.470) \\
\end{array}$ & $\begin{array}{c}0.802 \\
(0.457) \\
\end{array}$ & $\begin{array}{c}0.892 \\
(0.313) \\
\end{array}$ & $\begin{array}{c}0.711 \\
(0.514) \\
\end{array}$ \\
\hline & \multicolumn{6}{|c|}{ I-B) Dependent Variable: Ever Given Birth } & \multicolumn{6}{|c|}{$\begin{array}{l}\text { II-B) Dependent Variable: Birth status conditional } \\
\text { on not giving birth until that age }\end{array}$} \\
\hline Age $=15$ & $\begin{array}{c}1.154 \\
{[0.523]}\end{array}$ & $\begin{array}{c}1.194 \\
{[0.587]}\end{array}$ & $\begin{array}{c}0.743 \\
{[0.628]}\end{array}$ & $\begin{array}{c}0.744 \\
{[0.649]}\end{array}$ & $\begin{array}{c}1.220 \\
{[0.623]}\end{array}$ & $\begin{array}{c}0.640 \\
{[0.753]}\end{array}$ & $\begin{array}{c}0.617 \\
(0.392)\end{array}$ & $\begin{array}{c}0.595 \\
(0.420)\end{array}$ & $\begin{array}{c}0.143 \\
(0.169)\end{array}$ & $\begin{array}{c}0.135 \\
(0.169)\end{array}$ & $\begin{array}{c}0.594 \\
(0.436)\end{array}$ & $\begin{array}{c}0.071 \\
(0.121)\end{array}$ \\
\hline Age $=16$ & $\begin{array}{c}1.235 \\
{[0.337]}\end{array}$ & $\begin{array}{c}1.278 \\
{[0.356]}\end{array}$ & $\begin{array}{c}1.072 \\
{[0.479]}\end{array}$ & $\begin{array}{c}1.051 \\
{[0.458]}\end{array}$ & $\begin{array}{l}1.289 \\
{[0.363]}\end{array}$ & $\begin{array}{c}1.004 \\
{[0.576]}\end{array}$ & $\begin{array}{c}1.288 \\
(0.501)\end{array}$ & $\begin{array}{c}1.306 \\
(0.513)\end{array}$ & $\begin{array}{c}1.357 \\
(0.894)\end{array}$ & $\begin{array}{c}1.271 \\
(0.801)\end{array}$ & $\begin{array}{c}1.302 \\
(0.511)\end{array}$ & $\begin{array}{c}1.298 \\
(1.067)\end{array}$ \\
\hline Age $=17$ & $\begin{array}{c}1.534^{*} \\
{[0.368]}\end{array}$ & $\begin{array}{c}1.537^{*} \\
{[0.378]}\end{array}$ & $\begin{array}{c}1.152 \\
{[0.432]}\end{array}$ & $\begin{array}{c}1.125 \\
{[0.417]}\end{array}$ & $\begin{array}{c}1.536^{*} \\
{[0.380]}\end{array}$ & $\begin{array}{c}1.065 \\
{[0.516]}\end{array}$ & $\begin{array}{l}1.848^{*} \\
(0.629)\end{array}$ & $\begin{array}{c}1.728 \\
(0.584)\end{array}$ & $\begin{array}{c}1.174 \\
(0.625)\end{array}$ & $\begin{array}{c}1.154 \\
(0.622)\end{array}$ & $\begin{array}{c}1.712 \\
(0.576)\end{array}$ & $\begin{array}{c}1.083 \\
(0.743)\end{array}$ \\
\hline Age $=18$ & $\begin{array}{c}1.252 \\
{[0.268]}\end{array}$ & $\begin{array}{c}1.254 \\
{[0.271]}\end{array}$ & $\begin{array}{c}0.933 \\
{[0.325]}\end{array}$ & $\begin{array}{c}0.910 \\
{[0.308]}\end{array}$ & $\begin{array}{c}1.250 \\
{[0.270]}\end{array}$ & $\begin{array}{c}0.867 \\
{[0.364]}\end{array}$ & $\begin{array}{c}0.905 \\
(0.311)\end{array}$ & $\begin{array}{c}0.905 \\
(0.314)\end{array}$ & $\begin{array}{c}0.688 \\
(0.389)\end{array}$ & $\begin{array}{c}0.680 \\
(0.370)\end{array}$ & $\begin{array}{c}0.902 \\
(0.310)\end{array}$ & $\begin{array}{c}0.674 \\
(0.440)\end{array}$ \\
\hline Age $=19$ & $\begin{array}{c}1.068 \\
{[0.186]}\end{array}$ & $\begin{array}{c}1.061 \\
{[0.184]}\end{array}$ & $\begin{array}{c}0.972 \\
{[0.253]}\end{array}$ & $\begin{array}{c}0.984 \\
{[0.260]}\end{array}$ & $\begin{array}{c}1.061 \\
{[0.185]}\end{array}$ & $\begin{array}{c}1.026 \\
{[0.332]}\end{array}$ & $\begin{array}{c}0.818 \\
(0.210) \\
\end{array}$ & $\begin{array}{c}0.803 \\
(0.200) \\
\end{array}$ & $\begin{array}{c}1.057 \\
(0.433) \\
\end{array}$ & $\begin{array}{c}1.192 \\
(0.507) \\
\end{array}$ & $\begin{array}{c}0.809 \\
(0.202) \\
\end{array}$ & $\begin{array}{c}1.413 \\
(0.755) \\
\end{array}$ \\
\hline
\end{tabular}

Notes: The data come from the 2013 DHS. The data are restricted to cohorts born before 1985 (i.e. those that are not affected by the 1997 education reform). In this range of birth cohorts, we take the 1964 to 1979 birth cohorts excluding the 1971 and 1972 birth cohorts (7-year intervals around the donut-hole). The counterfactual cut-off value of month-year of birth for the policy variable is January 1972. The number of observations is 3,606 in panels (I-A) and (I-B), 18,127 in panel (II-A), and 16,476 in panel (II-B). Each cell comes from a separate logistic regression of ever-married status in panel (I-A) and of ever-given-birth status in panel (I-B) at the specified age on the policy variable and the specified time trends in month and year of birth. The estimates in each column come from a separate logistic regression of marriage hazard status in panel (II-A) and of birth hazard status in panel (II-B) on the interactions of the policy variable with each age dummy and the interactions of the specified time trends in month and year of birth with each age dummy. Odds ratios and their standard errors are given as estimated parameters. The standard errors are clustered at the month-and-year-ofbirth level. Statistical significance is $* * *$ at 1 percent level, $* *$ at 5 percent level, $*$ at 10 percent level. 
Table A8: Policy Effect on Marriage and First-Birth by Age (Odds Ratios) - 10-year Birth

Intervals on Both Sides of the Donut-hole; Year of Birth as the Running Variable

\begin{tabular}{|c|c|c|c|c|c|c|c|}
\hline & \multicolumn{4}{|c|}{ Single Time Trend } & \multicolumn{2}{|c|}{ Split Time Trends } & \multirow[b]{2}{*}{ Obs. } \\
\hline & $\begin{array}{l}\text { Linear } \\
(1)\end{array}$ & $\begin{array}{c}\text { Quadratic } \\
\text { (2) }\end{array}$ & $\begin{array}{c}\text { Cubic } \\
(3)\end{array}$ & $\begin{array}{c}\text { Quartic } \\
\text { (4) }\end{array}$ & $\begin{array}{c}\text { Linear } \\
(5)\end{array}$ & $\begin{array}{c}\text { Quadratic } \\
(6)\end{array}$ & \\
\hline \multicolumn{8}{|c|}{ A) Dependent Variable: Ever Married } \\
\hline Age $=12$ & $\begin{array}{c}0.729 \\
{[0.365]}\end{array}$ & $\begin{array}{c}0.630 \\
{[0.448]}\end{array}$ & $\begin{array}{c}1.435 \\
{[1.301]}\end{array}$ & $\begin{array}{c}2.567 \\
{[2.153]}\end{array}$ & $\begin{array}{c}0.560 \\
{[0.367]}\end{array}$ & $\begin{array}{c}2,216 \\
{[2.397]}\end{array}$ & 12,863 \\
\hline Age $=13$ & $\begin{array}{l}0.482 * \\
{[0.180]}\end{array}$ & $\begin{array}{c}0.593 \\
{[0.269]}\end{array}$ & $\begin{array}{c}0.643 \\
{[0.446]}\end{array}$ & $\begin{array}{c}0.919 \\
{[0.585]}\end{array}$ & $\begin{array}{c}0.610 \\
{[0.238]}\end{array}$ & $\begin{array}{c}0.888 \\
{[0.599]}\end{array}$ & 12,863 \\
\hline Age $=14$ & $\begin{array}{c}0.486^{* * *} \\
{[0.110]}\end{array}$ & $\begin{array}{l}0.629^{*} \\
{[0.149]}\end{array}$ & $\begin{array}{l}0.557^{*} \\
{[0.185]}\end{array}$ & $\begin{array}{l}0.555^{*} \\
{[0.173]}\end{array}$ & $\begin{array}{l}0.625^{* *} \\
{[0.141]}\end{array}$ & $\begin{array}{l}0.485^{* *} \\
{[0.177]}\end{array}$ & 12,863 \\
\hline Age $=15$ & $\begin{array}{c}0.510^{* * *} \\
{[0.106]}\end{array}$ & $\begin{array}{l}0.702 * * \\
{[0.115]}\end{array}$ & $\begin{array}{c}0.695 \\
{[0.163]}\end{array}$ & $\begin{array}{c}0.656 \\
{[0.179]}\end{array}$ & $\begin{array}{l}0.682 * * \\
{[0.105]}\end{array}$ & $\begin{array}{c}0.666 \\
{[0.192]}\end{array}$ & 12,863 \\
\hline Age $=16$ & $\begin{array}{l}0.707 * * \\
{[0.100]}\end{array}$ & $\begin{array}{c}0.839 \\
{[0.107]}\end{array}$ & $\begin{array}{l}0.736^{* *} \\
{[0.108]}\end{array}$ & $\begin{array}{c}0.657 * * * \\
{[0.101]}\end{array}$ & $\begin{array}{c}0.818 \\
{[0.107]}\end{array}$ & $\begin{array}{l}0.649 * * \\
{[0.125]}\end{array}$ & 12,417 \\
\hline Age $=17$ & $\begin{array}{c}1.019 \\
{[0.120]}\end{array}$ & $\begin{array}{c}1.137 \\
{[0.133]}\end{array}$ & $\begin{array}{c}1.183 \\
{[0.212]}\end{array}$ & $\begin{array}{c}1.012 \\
{[0.171]}\end{array}$ & $\begin{array}{c}1.093 \\
{[0.131]}\end{array}$ & $\begin{array}{c}1.033 \\
{[0.203]}\end{array}$ & 11,564 \\
\hline Age $=18$ & $\begin{array}{c}0.955 \\
{[0.075]}\end{array}$ & $\begin{array}{c}1,015 \\
{[0.137]}\end{array}$ & $\begin{array}{c}0.939 \\
{[0.145]}\end{array}$ & $\begin{array}{l}0.774 * \\
{[0.113]}\end{array}$ & $\begin{array}{c}0.990 \\
{[0.125]}\end{array}$ & $\begin{array}{l}0.732 * * \\
{[0.089]}\end{array}$ & 10,728 \\
\hline Age $=19$ & $\begin{array}{c}0.984 \\
{[0.059]}\end{array}$ & $\begin{array}{c}0.993 \\
{[0.058]}\end{array}$ & $\begin{array}{c}1.048 \\
{[0.087]}\end{array}$ & $\begin{array}{c}1.036 \\
{[0.102]}\end{array}$ & $\begin{array}{c}0.978 \\
{[0.050]}\end{array}$ & $\begin{array}{c}0.998 \\
{[0.066]}\end{array}$ & 9,859 \\
\hline \multicolumn{8}{|c|}{ B) Dependent Variable: Ever Given Birth } \\
\hline Age $=13$ & $\begin{array}{c}1.077 \\
{[0.913]}\end{array}$ & $\begin{array}{c}2.228 \\
{[2.055]}\end{array}$ & $\begin{array}{c}1.263 \\
{[1.412]}\end{array}$ & $\begin{array}{c}0.792 \\
{[1.154]}\end{array}$ & $\begin{array}{c}2.180 \\
{[1.446]}\end{array}$ & $\begin{array}{c}0.125 \\
{[0.241]}\end{array}$ & 12,863 \\
\hline Age $=14$ & $\begin{array}{c}0.470 \\
{[0.326]}\end{array}$ & $\begin{array}{c}0.778 \\
{[0.572]}\end{array}$ & $\begin{array}{c}0.574 \\
{[0.568]}\end{array}$ & $\begin{array}{c}0.299 \\
{[0.397]}\end{array}$ & $\begin{array}{c}0.781 \\
{[0.501]}\end{array}$ & $\begin{array}{c}0.351 \\
{[0.456]}\end{array}$ & 12,863 \\
\hline Age $=15$ & $\begin{array}{c}0.558 \\
{[0.212]}\end{array}$ & $\begin{array}{l}0.498^{* *} \\
{[0.177]}\end{array}$ & $\begin{array}{l}0.378 * * \\
{[0.166]}\end{array}$ & $\begin{array}{c}0.303 * * * \\
{[0.140]}\end{array}$ & $\begin{array}{l}0.502^{*} \\
{[0.179]}\end{array}$ & $\begin{array}{l}0.254^{* *} \\
{[0.157]}\end{array}$ & 12,863 \\
\hline Age $=16$ & $\begin{array}{c}0.583^{* * *} \\
{[0.115]}\end{array}$ & $\begin{array}{l}0.711^{*} \\
{[0.147]}\end{array}$ & $\begin{array}{l}0.523 * * \\
{[0.133]}\end{array}$ & $\begin{array}{c}0.430 * * * \\
{[0.123]}\end{array}$ & $\begin{array}{l}0.708^{*} \\
{[0.130]}\end{array}$ & $\begin{array}{c}0.406^{* * *} \\
{[0.128]}\end{array}$ & 12,417 \\
\hline Age $=17$ & $\begin{array}{c}0.695 * * * \\
{[0.087]}\end{array}$ & $\begin{array}{l}0.725^{* *} \\
{[0.111]}\end{array}$ & $\begin{array}{l}0.696^{* *} \\
{[0.107]}\end{array}$ & $\begin{array}{c}0.554 * * * \\
{[0.105]}\end{array}$ & $\begin{array}{l}0.712^{* *} \\
{[0.105]}\end{array}$ & $\begin{array}{l}0.555^{* *} \\
{[0.166]}\end{array}$ & 11,558 \\
\hline Age $=18$ & $\begin{array}{c}0.898 \\
{[0.070]}\end{array}$ & $\begin{array}{c}1.027 \\
{[0.134]}\end{array}$ & $\begin{array}{c}1.033 \\
{[0.145]}\end{array}$ & $\begin{array}{l}0.735^{*} \\
{[0.123]}\end{array}$ & $\begin{array}{c}0.974 \\
{[0.118]}\end{array}$ & $\begin{array}{c}0.751 \\
{[0.164]}\end{array}$ & 10,718 \\
\hline Age $=19$ & $\begin{array}{l}0.888 * * \\
{[0.049]}\end{array}$ & $\begin{array}{l}0.901 * \\
{[0.052]}\end{array}$ & $\begin{array}{c}0.946 \\
{[0.081]}\end{array}$ & $\begin{array}{c}0.901 \\
{[0.089]}\end{array}$ & $\begin{array}{l}0.882 * * \\
{[0.044]}\end{array}$ & $\begin{array}{c}0.867 \\
{[0.075]}\end{array}$ & 9,844 \\
\hline
\end{tabular}

Notes: The sample includes observations from both 2008 and 2013 DHS. The sample is restricted to 10-year intervals around the donuthole; i.e., it includes 1976-1985 and 1988-97 birth cohorts. The policy dummy is one when year of birth is greater than 1987. Each cell comes from a separate logistic regression of ever-married status at the specified age in panel (A) and of ever-given-birth status at the specified age in panel (B) on the policy variable as well as the specified time trends. Odds ratios and their standard errors are given as estimated parameters. In the first 4 columns, single time trends up to quartic polynomials are fitted, whereas separate polynomials are fitted on either side of the discontinuity in columns (5) and (6). The standard errors are clustered at the year-of-birth level. Statistical significance is *** at 1 percent level, ** at 5 percent level, * at 10 percent level. 
Table A9: Policy Effect on Marriage and First-Birth by Age (Odds Ratios) - 5-year Birth Intervals on Both Sides of the Donut-hole; Year of Birth as the Running Variable

\begin{tabular}{|c|c|c|c|c|c|c|c|c|}
\hline & \multirow{2}{*}{\multicolumn{3}{|c|}{$\begin{array}{l}\text { Split Time } \\
\text { Trends } \\
\text { Linear } \\
(3)\end{array}$}} & \multirow[b]{2}{*}{$\begin{array}{l}\text { Obs. } \\
(4)\end{array}$} & \multicolumn{2}{|c|}{ Single Time Trend } & \multirow{2}{*}{$\begin{array}{c}\text { Split Time } \\
\text { Trends } \\
\begin{array}{c}\text { Linear } \\
(7)\end{array}\end{array}$} & \multirow[b]{2}{*}{$\begin{array}{l}\text { Obs. } \\
(8)\end{array}$} \\
\hline & & & & & $\begin{array}{c}\text { Linear } \\
(5)\end{array}$ & $\begin{array}{c}\text { Quadratic } \\
(6)\end{array}$ & & \\
\hline & \multicolumn{4}{|c|}{ A) Dependent Variable: Ever Married } & \multicolumn{4}{|c|}{ B) Dependent Variable: Ever Given Birth } \\
\hline Age $=12$ & $\begin{array}{c}1.472 \\
{[0.844]}\end{array}$ & $\begin{array}{c}0.800 \\
{[0.468]}\end{array}$ & $\begin{array}{c}0.855 \\
{[0.525]}\end{array}$ & 7,310 & -- & -- & -- & -- \\
\hline Age $=13$ & $\begin{array}{c}0.518 \\
{[0.381]}\end{array}$ & $\begin{array}{c}0.603 \\
{[0.323]}\end{array}$ & $\begin{array}{c}0.655 \\
{[0.309]}\end{array}$ & 7,310 & $\begin{array}{c}1.640 \\
{[2.544]}\end{array}$ & $\begin{array}{c}1.435 \\
{[2.196]}\end{array}$ & $\begin{array}{c}1.590 \\
{[2.261]}\end{array}$ & 7,310 \\
\hline Age $=14$ & $\begin{array}{c}0.397 * * * \\
{[0.087]}\end{array}$ & $\begin{array}{c}0.446 * * * \\
{[0.085]}\end{array}$ & $\begin{array}{c}0.455 * * * \\
{[0.089]}\end{array}$ & 7,310 & $\begin{array}{c}0.327 \\
{[0.403]}\end{array}$ & $\begin{array}{c}0.414 \\
{[0.470]}\end{array}$ & $\begin{array}{c}0.522 \\
{[0.458]}\end{array}$ & 7,310 \\
\hline Age $=15$ & $\begin{array}{c}0.430 * * * \\
{[0.114]}\end{array}$ & $\begin{array}{c}0.483 * * * \\
{[0.113]}\end{array}$ & $\begin{array}{c}0.484 * * * \\
{[0.106]}\end{array}$ & 7,310 & $\begin{array}{l}0.431 * \\
{[0.191]}\end{array}$ & $\begin{array}{c}0.326 * * \\
{[0.180]}\end{array}$ & $\begin{array}{c}0.359 * \\
{[0.192]}\end{array}$ & 7,310 \\
\hline Age $=16$ & $\begin{array}{c}0.698 * * \\
{[0.110]}\end{array}$ & $\begin{array}{c}0.756 * * \\
{[0.094]}\end{array}$ & $\begin{array}{c}0.747 * * \\
{[0.095]}\end{array}$ & 7,310 & $\begin{array}{c}0.400 * * * \\
{[0.111]}\end{array}$ & $\begin{array}{c}0.457 * * * \\
{[0.087]}\end{array}$ & $\begin{array}{c}0.460 * * * \\
{[0.076]}\end{array}$ & 7,310 \\
\hline Age $=17$ & $\begin{array}{c}1.099 \\
{[0.200]}\end{array}$ & $\begin{array}{c}1.092 \\
{[0.199]}\end{array}$ & $\begin{array}{c}1.084 \\
{[0.187]}\end{array}$ & 6,867 & $\begin{array}{c}0.580 * * * \\
{[0.073]}\end{array}$ & $\begin{array}{c}0.604 * * \\
{[0.137]}\end{array}$ & $\begin{array}{c}0.599 * * \\
{[0.129]}\end{array}$ & 6,862 \\
\hline Age $=18$ & $\begin{array}{c}0.936 \\
{[0.125]}\end{array}$ & $\begin{array}{c}0.917 \\
{[0.111]}\end{array}$ & $\begin{array}{c}0.913 \\
{[0.098]}\end{array}$ & 6,438 & $\begin{array}{c}0.855 \\
{[0.110]}\end{array}$ & $\begin{array}{c}0.844 \\
{[0.095]}\end{array}$ & $\begin{array}{c}0.839 \\
{[0.091]}\end{array}$ & 6,432 \\
\hline Age $=19$ & $\begin{array}{c}1.011 \\
{[0.099]}\end{array}$ & $\begin{array}{c}1.001 \\
{[0.080]}\end{array}$ & $\begin{array}{c}0.997 \\
{[0.071]}\end{array}$ & 5,969 & $\begin{array}{c}0.897 \\
{[0.082]}\end{array}$ & $\begin{array}{c}0.881^{*} \\
{[0.063]}\end{array}$ & $\begin{array}{c}0.877 * * \\
{[0.058]}\end{array}$ & 5,964 \\
\hline
\end{tabular}

Notes: The sample includes observations from both 2008 and 2013 DHS. The sample is restricted to 5-year intervals around the donut-hole; i.e., it includes 1981-1985 and 1988-92 birth cohorts. The policy dummy is one when year of birth is greater than 1987. Each cell comes from a separate logistic regression of ever-married status at the specified age in panel (A) and of ever-given-birth status at the specified age in panel (B) on the policy variable as well as the specified time trends. Odds ratios and their standard errors are given as estimated parameters. The standard errors are clustered at the year-of-birth level. Statistical significance is $* * *$ at 1 percent level, ** at 5 percent level, * at 10 percent level. 
Table A10: Policy Effect on Number of Children - Sample (B3) and Month-Year of Birth as

Running Variable, OLS results

\begin{tabular}{|c|c|c|c|c|c|c|c|c|c|}
\hline \multirow[b]{2}{*}{$\begin{array}{l}\text { Degree of } \\
\text { Polynomial } \\
\end{array}$} & \multicolumn{6}{|c|}{ Single Time Trend } & \multicolumn{3}{|c|}{ Split Time Trends } \\
\hline & $\begin{array}{l}\text { One } \\
(1)\end{array}$ & $\begin{array}{l}\text { Two } \\
(2) \\
\end{array}$ & $\begin{array}{l}\text { Three } \\
(3)\end{array}$ & $\begin{array}{c}\text { Four } \\
(4) \\
\end{array}$ & $\begin{array}{c}\text { Five } \\
(5)\end{array}$ & $\begin{array}{l}\text { Six } \\
(6) \\
\end{array}$ & $\begin{array}{l}\text { One } \\
(7)\end{array}$ & $\begin{array}{l}\text { Two } \\
(8) \\
\end{array}$ & $\begin{array}{c}\text { Three } \\
(9)\end{array}$ \\
\hline \multicolumn{10}{|c|}{ A) Dependent Variable: Ever Married } \\
\hline Age $=15$ & $\begin{array}{c}0.002 \\
{[0.004]}\end{array}$ & $\begin{array}{c}0.002 \\
{[0.004]}\end{array}$ & $\begin{array}{c}0.008 \\
{[0.006]}\end{array}$ & $\begin{array}{c}0.008 \\
{[0.006]}\end{array}$ & $\begin{array}{c}0.004 \\
{[0.009]}\end{array}$ & $\begin{array}{c}0.004 \\
{[0.009]}\end{array}$ & $\begin{array}{c}0.002 \\
{[0.004]}\end{array}$ & $\begin{array}{c}0.009 \\
{[0.008]}\end{array}$ & $\begin{array}{c}-0.003 \\
{[0.014]}\end{array}$ \\
\hline Age $=16$ & $\begin{array}{l}-0.005 \\
{[0.007]}\end{array}$ & $\begin{array}{c}-0.005 \\
{[0.007]}\end{array}$ & $\begin{array}{c}-0.005 \\
{[0.011]}\end{array}$ & $\begin{array}{c}-0.006 \\
{[0.011]}\end{array}$ & $\begin{array}{c}0.000 \\
{[0.015]}\end{array}$ & $\begin{array}{c}-0.001 \\
{[0.014]}\end{array}$ & $\begin{array}{c}-0.005 \\
{[0.007]}\end{array}$ & $\begin{array}{c}-0.006 \\
{[0.013]}\end{array}$ & $\begin{array}{c}-0.005 \\
{[0.024]}\end{array}$ \\
\hline Age $=17$ & $\begin{array}{c}-0.015 \\
{[0.014]}\end{array}$ & $\begin{array}{c}-0.017 \\
{[0.013]}\end{array}$ & $\begin{array}{c}-0.007 \\
{[0.019]}\end{array}$ & $\begin{array}{c}-0.016 \\
{[0.017]}\end{array}$ & $\begin{array}{c}-0.019 \\
{[0.025]}\end{array}$ & $\begin{array}{c}-0.032 \\
{[0.023]}\end{array}$ & $\begin{array}{c}-0.017 \\
{[0.013]}\end{array}$ & $\begin{array}{c}-0.015 \\
{[0.021]}\end{array}$ & $\begin{array}{c}-0.046 \\
{[0.040]}\end{array}$ \\
\hline Age $=18$ & $\begin{array}{c}-0.034 \\
{[0.021]}\end{array}$ & $\begin{array}{c}-0.038^{*} \\
{[0.021]}\end{array}$ & $\begin{array}{c}-0.043 \\
{[0.031]}\end{array}$ & $\begin{array}{c}-0.092 * * * \\
{[0.028]}\end{array}$ & $\begin{array}{l}-0.076^{*} \\
{[0.039]}\end{array}$ & $\begin{array}{c}-0.103 * * \\
{[0.040]}\end{array}$ & $\begin{array}{l}-0.040 * \\
{[0.021]}\end{array}$ & $\begin{array}{c}-0.097 * * * \\
{[0.033]}\end{array}$ & $\begin{array}{l}-0.121 * \\
{[0.063]}\end{array}$ \\
\hline Age $=19$ & $\begin{array}{c}-0.003 \\
{[0.031]}\end{array}$ & $\begin{array}{c}-0.021 \\
{[0.032]}\end{array}$ & $\begin{array}{c}0.024 \\
{[0.043]}\end{array}$ & $\begin{array}{c}-0.031 \\
{[0.048]}\end{array}$ & $\begin{array}{c}-0.007 \\
{[0.057]}\end{array}$ & $\begin{array}{c}0.005 \\
{[0.076]}\end{array}$ & $\begin{array}{c}-0.024 \\
{[0.031]}\end{array}$ & $\begin{array}{c}-0.021 \\
{[0.054]}\end{array}$ & $\begin{array}{c}-0.031 \\
{[0.108]}\end{array}$ \\
\hline Age $=20$ & $\begin{array}{c}0.008 \\
{[0.038]}\end{array}$ & $\begin{array}{c}-0.015 \\
{[0.044]}\end{array}$ & $\begin{array}{c}0.057 \\
{[0.053]}\end{array}$ & $\begin{array}{c}0.028 \\
{[0.071]}\end{array}$ & $\begin{array}{c}0.018 \\
{[0.074]}\end{array}$ & $\begin{array}{c}0.026 \\
{[0.112]}\end{array}$ & $\begin{array}{c}-0.023 \\
{[0.042]}\end{array}$ & $\begin{array}{c}0.043 \\
{[0.079]}\end{array}$ & $\begin{array}{c}-0.234 \\
{[0.172]}\end{array}$ \\
\hline
\end{tabular}

Notes: The data come from the 2013 DHS. The sample includes 10 years on the left hand side of the donut-hole; on the right hand side of the donut-hole, the sample includes 10 years for age 15, 9 years for age 16, 8 years for age 17,7 years for age 18,6 years for age 19, and 5 years for age 20. The number of observations is 5,946 in all cells for age 15, 5,921 for age 16, 5,589 for age 17, 5,273 for age 18, 4,970 for age 19, and 4,685 for age 20. The policy dummy is one when year of birth is greater than 1987. Each cell comes from a separate OLS regression of the number of children born by the specified age on the policy variable and the specified time trends in month and year of birth. The standard errors are clustered at the month-year of birth level. Statistical significance is $* * *$ at 1 percent level, ** at 5 percent level, * at 10 percent level. 


\section{APPENDIX B: SURVIVAL ANALYSIS}

We examine the policy effects on the timing of marriage and first-birth using duration analysis. For this purpose, we construct event histories of time to marriage and time to first-birth. Women enter the risk set of marriage at age 14 and the risk set of first-birth at age 15 . Women who get married before age 14 are dropped from the marriage sample, and women who give birth before age 15 are dropped from the birth sample. Individuals exit the risk set when they get married in the first case and give their first-birth in the second case. The duration is censored on the right if women do not marry/give birth until age 19 or until their current age at the time of the survey, whichever comes earlier. Unlike the level analysis, where marriage and first-birth outcomes are examined at each age separately, the data are pooled over ages in the timing analysis. Basic descriptive statistics on personage level characeristics in the survival samples (B1) to (B3) are given in Table B1.

The estimates for the policy effect on the marriage and first-birth hazard rates are presented in Table B2 for samples (B1) and (B2) and in Table B3 for sample (B3). ${ }^{21}$ The estimates for the marriage hazard rate, given in panel A of each of these tables, are quite stable over the time windows and the specifications. A key finding here is that the policy in fact increases the marriage hazard rate at age 17. (This is statistically significant at the 1 percent level in almost all specifications in both tables.) Moreover, the magnitude of the effect is substantial. With more flexible specifications - the rightmost columns, given the time-window and whether or not the time trend is split-the estimates show that the policy increases the odds of the marriage hazard by a factor of more than four. The policy effects on the marriage hazard rate in earlier ages (ages 14 to 16) are all negative, apart from those for age 16 with the least flexible specifications and wide time-window in Table B3. However, the

\footnotetext{
${ }^{21}$ The results for sample (A) are given in Table B4. While the findings are similar, statistical significance is generally lower-particularly for specifications with split time trends.
} 
negative effect is statistically significant only for age 15 in both tables. The negative effect is not as strong in magnitude at age 16; while the negative effect is strong in magnitude at age 14, it is statistically insignificant, presumably due to the low incidence of marriage at this age. There is no evidence of a policy effect on the marriage hazard rates at ages 18 or 19.

When we examine the policy effect on the first-birth hazard rate, given in panel (B) of Tables B2 and B3, we see evidence for a positive policy effect on the hazard rate at age 18 in both tables. According to the estimates in Table B2, the policy increases the first-birth hazard rate at age 18 by a factor more than 2. In addition, the odds ratios for age 19 in both tables are substantially higher than one, suggesting a positive policy effect at this age as well. However, its statistical significance is not robust across different specifications. At earlier ages, 15 to 17, the odds ratios are all less than one in both Tables B2 and B3. However, the results on statistical significance are mixed. With sample (B1) in Table B2, there is evidence of a negative policy effect on the first-birth hazard rate at ages 15 and 16 , but not age 17. On the other hand, with sample (B2) in Table B2, there is strong evidence of a negative policy effect on the first-birth hazard rate at age 17 but weaker evidence for ages 15 and 16. Also with the more flexible specifications estimated with sample (B3) in Table B3, there is evidence of a negative policy effect on the first-birth hazard rate at ages 16 and 17, but no robust evidence exists at age 15 .

In summary, while the marriage hazard rate at ages 15 and below-when students are incarcerated in school—decreases with the policy, once the students are out of school at age 17, they are more likely to get married as a result of the policy. Consequently, after age 17, the probability of being ever married reverts to its baseline (no policy) levels as shown in Tables 2 and 3. Consistent with the rigid sequence of marriage and birth events, while the policy decreases the first-birth hazard rate at ages 
15 to 17 , it increases it after age 18 . As a result, the probability of ever giving birth by age 18 reverts to its pre-policy level. 
Table B1: Descriptive Statistics - Person-Age Level Characteristics for Survival Analysis Samples

\begin{tabular}{|c|c|c|c|c|c|c|c|c|c|c|c|}
\hline & \multicolumn{5}{|c|}{ A) Marriage Sample } & & \multicolumn{5}{|c|}{ B) First-birth Sample } \\
\hline & Mean & S.D. & Min. & Max. & No. Obs. & & Mean & S.D. & Min. & Max. & No. Obs. \\
\hline 1) Sample B1 & & & & & & 1) Sample B1 & & & & & \\
\hline Year of Birth & 1986.4 & 3.2 & 1982 & 1991 & 12,533 & Year of Birth & 1986.4 & 3.2 & 1982 & 1991 & 11,055 \\
\hline Year & 2002.8 & 3.6 & 1996 & 2010 & 12,533 & Year & 2003.3 & 3.5 & 1997 & 2010 & 11,055 \\
\hline Age & 16.34 & 1.68 & 14 & 19 & 12,533 & Age & 16.93 & 1.40 & 15 & 19 & 11,055 \\
\hline Policy & 0.495 & 0.500 & 0 & 1 & 12,533 & Policy & 0.492 & 0.500 & 0 & 1 & 11,055 \\
\hline Married & 0.063 & 0.243 & 0 & 1 & 12,533 & Given Birth & 0.043 & 0.202 & 0 & 1 & 11,055 \\
\hline Trend (in months) & -1.17 & 38.49 & -59.5 & 59.5 & 12,533 & Trend (in months) & -1.46 & 38.49 & -59.5 & 59.5 & 11,055 \\
\hline 2) Sample B2 & & & & & & 2) Sample B2 & & & & & \\
\hline Year of Birth & 1986.4 & 4.9 & 1979 & 1994 & 21,769 & Year of Birth & 1986.3 & 4.9 & 1979 & 1994 & 19,221 \\
\hline Year & 2002.7 & 5.200 & 1993 & 2013 & 21,769 & Year & 2003.3 & 5.1 & 1994 & 2013 & 19,221 \\
\hline Age & 16.33 & 1.68 & 14 & 19 & 21,769 & Age & 16.93 & 1.40 & 15 & 19 & 19,221 \\
\hline Policy & 0.490 & 0.500 & 0 & 1 & 21,769 & Policy & 0.485 & 0.500 & 0 & 1 & 19,221 \\
\hline Married & 0.063 & 0.244 & 0 & 1 & 21,769 & Given Birth & 0.043 & 0.204 & 0 & 1 & 19,221 \\
\hline Trend (in months) & -1.75 & 58.88 & -95.5 & 95.5 & 21,769 & Trend (in months) & -2.47 & 58.86 & -95.5 & 95.5 & 19,221 \\
\hline 3) Sample B3 & & & & & & 3) Sample B3 & & & & & \\
\hline Year of Birth & 1986.1 & 6.5 & 1976 & 1997 & 30,151 & Year of Birth & 1985.8 & 6.4 & 1976 & 1997 & 26,220 \\
\hline Year & 2002.3 & 6.6 & 1990 & 2013 & 30,151 & Year & 2002.6 & 6.4 & 1991 & 2013 & 26,220 \\
\hline Age & 16.22 & 1.65 & 14 & 19 & 30,151 & Age & 16.83 & 1.39 & 15 & 19 & 26,220 \\
\hline Policy & 0.475 & 0.499 & 0 & 1 & 30,151 & Policy & 0.460 & 0.498 & 0 & 1 & 26,220 \\
\hline Married & 0.061 & 0.239 & 0 & 1 & 30,151 & Given Birth & 0.043 & 0.203 & 0 & 1 & 26,220 \\
\hline Trend (in months) & -5.68 & 77.73 & -131.5 & 131.5 & 30,151 & Trend (in months) & -8.77 & 76.69 & -131.5 & 131.5 & 26,220 \\
\hline
\end{tabular}

Notes: The samples are drawn from 15- to 49-year-old individuals in the 2008 and 2013 DHS surveys. All samples exclude the 1986 and 1987 birth cohorts. 
Table B2: Policy Effect on Marriage and First-Birth Hazard Rates by Age, Odds Ratios Month-Year of Birth as Running Variable, 4-Year and 7-Year Intervals around the Donut-

\section{hole}

\begin{tabular}{|c|c|c|c|c|c|c|c|c|c|}
\hline \multirow[b]{3}{*}{$\begin{array}{l}\text { Degree of } \\
\text { Polynomial } \\
\end{array}$} & \multicolumn{3}{|c|}{ I) 4-Year Intervals } & \multicolumn{6}{|c|}{ II) 7-Year Intervals } \\
\hline & \multicolumn{2}{|c|}{$\begin{array}{c}\text { Single Time } \\
\text { Trend }\end{array}$} & \multirow{2}{*}{$\begin{array}{c}\begin{array}{c}\text { Split } \\
\text { Trends }\end{array} \\
\begin{array}{c}\text { One } \\
(3)\end{array} \\
\end{array}$} & \multicolumn{4}{|c|}{ Single Time Trend } & \multicolumn{2}{|c|}{ Split Time Trends } \\
\hline & $\begin{array}{l}\text { One } \\
(1)\end{array}$ & $\begin{array}{l}\text { Two } \\
(2) \\
\end{array}$ & & $\begin{array}{l}\text { One } \\
(4)\end{array}$ & $\begin{array}{c}\text { Two } \\
(5) \\
\end{array}$ & $\begin{array}{c}\text { Three } \\
(6)\end{array}$ & $\begin{array}{c}\text { Four } \\
(7)\end{array}$ & $\begin{array}{c}\text { One } \\
(8) \\
\end{array}$ & $\begin{array}{l}\text { Two } \\
(9) \\
\end{array}$ \\
\hline \multicolumn{10}{|c|}{ A) Dependent Variable: Marriage status conditional on not being married until that age } \\
\hline Age $=14$ & $\begin{array}{c}0.292 \\
(0.377)\end{array}$ & $\begin{array}{c}0.205 \\
(0.285)\end{array}$ & $\begin{array}{c}0.218 \\
(0.298)\end{array}$ & $\begin{array}{c}0.690 \\
(0.567)\end{array}$ & $\begin{array}{c}0.553 \\
(0.485)\end{array}$ & $\begin{array}{c}0.273 \\
(0.368)\end{array}$ & $\begin{array}{c}0.114 \\
(0.184)\end{array}$ & $\begin{array}{c}0.557 \\
(0.482)\end{array}$ & $\begin{array}{c}0.083 \\
(0.162)\end{array}$ \\
\hline Age $=15$ & $\begin{array}{c}0.302 * * \\
(0.176)\end{array}$ & $\begin{array}{c}0.346 \\
(0.243)\end{array}$ & $\begin{array}{l}0.308^{*} \\
(0.220)\end{array}$ & $\begin{array}{c}0.308 * * * \\
(0.128)\end{array}$ & $\begin{array}{c}0.267 * * \\
(0.137)\end{array}$ & $\begin{array}{l}0.253 * \\
(0.179)\end{array}$ & $\begin{array}{c}0.237^{*} \\
(0.189)\end{array}$ & $\begin{array}{c}0.259 * * \\
(0.137)\end{array}$ & $\begin{array}{l}0.177^{*} \\
(0.185)\end{array}$ \\
\hline Age $=16$ & $\begin{array}{c}0.826 \\
(0.401)\end{array}$ & $\begin{array}{c}0.796 \\
(0.398)\end{array}$ & $\begin{array}{c}0.796 \\
(0.398)\end{array}$ & $\begin{array}{c}0.640 \\
(0.197)\end{array}$ & $\begin{array}{c}0.624 \\
(0.199)\end{array}$ & $\begin{array}{c}0.876 \\
(0.464)\end{array}$ & $\begin{array}{c}0.831 \\
(0.467)\end{array}$ & $\begin{array}{c}0.620 \\
(0.200)\end{array}$ & $\begin{array}{c}0.914 \\
(0.653)\end{array}$ \\
\hline Age $=17$ & $\begin{array}{c}4.355^{* * * *} \\
(1.729)\end{array}$ & $\begin{array}{c}4.970 * * * \\
(2.042)\end{array}$ & $\begin{array}{c}5.300 * * * \\
(2.275)\end{array}$ & $\begin{array}{c}2.446 * * * \\
(0.670)\end{array}$ & $\begin{array}{c}2.459 * * * \\
(0.686)\end{array}$ & $\begin{array}{c}3.646^{* * * *} \\
(1.585)\end{array}$ & $\begin{array}{c}4.157 * * * \\
(1.974)\end{array}$ & $\begin{array}{c}2.507 * * * \\
(0.716)\end{array}$ & $\begin{array}{c}6.511 * * * \\
(4.296)\end{array}$ \\
\hline Age $=18$ & $\begin{array}{c}0.822 \\
(0.304)\end{array}$ & $\begin{array}{c}0.820 \\
(0.301)\end{array}$ & $\begin{array}{c}0.818 \\
(0.298)\end{array}$ & $\begin{array}{c}1.098 \\
(0.282)\end{array}$ & $\begin{array}{c}1.098 \\
(0.284)\end{array}$ & $\begin{array}{c}1.125 \\
(0.454)\end{array}$ & $\begin{array}{c}1.138 \\
(0.445)\end{array}$ & $\begin{array}{c}1.098 \\
(0.283)\end{array}$ & $\begin{array}{c}1.004 \\
(0.487)\end{array}$ \\
\hline Age $=19$ & $\begin{array}{r}1.105 \\
(0.486) \\
\end{array}$ & $\begin{array}{r}1.102 \\
(0.499) \\
\end{array}$ & $\begin{array}{c}1.098 \\
(0.498) \\
\end{array}$ & $\begin{array}{r}1.193 \\
(0.361) \\
\end{array}$ & $\begin{array}{r}1.257 \\
(0.364) \\
\end{array}$ & $\begin{array}{r}0.653 \\
(0.317) \\
\end{array}$ & $\begin{array}{c}0.605 \\
(0.315) \\
\end{array}$ & $\begin{array}{c}1.236 \\
(0.356) \\
\end{array}$ & $\begin{array}{c}0.583 \\
(0.399) \\
\end{array}$ \\
\hline \multicolumn{10}{|c|}{ B) Dependent Variable: Birth status conditional on not giving birth until that age } \\
\hline Age $=15$ & $\begin{array}{c}0.193 \\
(0.225)\end{array}$ & $\begin{array}{l}0.051 * \\
(0.081)\end{array}$ & $\begin{array}{l}0.053^{*} \\
(0.081)\end{array}$ & $\begin{array}{l}0.277^{*} \\
(0.194)\end{array}$ & $\begin{array}{c}0.139 * * \\
(0.117)\end{array}$ & $\begin{array}{c}0.192 \\
(0.272)\end{array}$ & $\begin{array}{c}0.071 \\
(0.115)\end{array}$ & $\begin{array}{c}0.116^{* *} \\
(0.108)\end{array}$ & $\begin{array}{c}0.035 \\
(0.073)\end{array}$ \\
\hline Age $=16$ & $\begin{array}{l}0.294 * \\
(0.197)\end{array}$ & $\begin{array}{l}0.192 * \\
(0.168)\end{array}$ & $\begin{array}{l}0.165^{*} \\
(0.155)\end{array}$ & $\begin{array}{c}0.517 \\
(0.271)\end{array}$ & $\begin{array}{c}0.425 \\
(0.273)\end{array}$ & $\begin{array}{l}0.281 * \\
(0.216)\end{array}$ & $\begin{array}{l}0.191 * \\
(0.177)\end{array}$ & $\begin{array}{c}0.404 \\
(0.269)\end{array}$ & $\begin{array}{c}0.083 * * \\
(0.105)\end{array}$ \\
\hline Age $=17$ & $\begin{array}{c}0.512 \\
(0.286)\end{array}$ & $\begin{array}{c}0.451 \\
(0.263)\end{array}$ & $\begin{array}{c}0.430 \\
(0.255)\end{array}$ & $\begin{array}{c}0.458 * * \\
(0.171)\end{array}$ & $\begin{array}{c}0.443 * * \\
(0.175)\end{array}$ & $\begin{array}{l}0.316^{*} \\
(0.190)\end{array}$ & $\begin{array}{c}0.240 * * \\
(0.161)\end{array}$ & $\begin{array}{c}0.430 * * \\
(0.175)\end{array}$ & $\begin{array}{c}0.160 * * \\
(0.135)\end{array}$ \\
\hline Age $=18$ & $\begin{array}{l}2.343 * \\
(1.062)\end{array}$ & $\begin{array}{l}2.358 * \\
(1.075)\end{array}$ & $\begin{array}{l}2.393 * \\
(1.109)\end{array}$ & $\begin{array}{c}1.956 * * \\
(0.586)\end{array}$ & $\begin{array}{c}1.947 * * \\
(0.556)\end{array}$ & $\begin{array}{c}2.615^{* *} \\
(1.263)\end{array}$ & $\begin{array}{l}2.655^{*} \\
(1.325)\end{array}$ & $\begin{array}{c}1.924 * * \\
(0.554)\end{array}$ & $\begin{array}{l}3.564^{*} \\
(2.318)\end{array}$ \\
\hline Age $=19$ & $\begin{array}{c}1.658 \\
(0.667)\end{array}$ & $\begin{array}{c}1.665 \\
(0.651)\end{array}$ & $\begin{array}{c}1.643 \\
(0.633)\end{array}$ & $\begin{array}{l}1.710 * \\
(0.468)\end{array}$ & $\begin{array}{c}1.743 * * \\
(0.463)\end{array}$ & $\begin{array}{c}1.655 \\
(0.757)\end{array}$ & $\begin{array}{c}1.678 \\
(0.759)\end{array}$ & $\begin{array}{c}1.732 * * \\
(0.456)\end{array}$ & $\begin{array}{c}1.618 \\
(0.907)\end{array}$ \\
\hline
\end{tabular}

Notes: The data come from the 2013 DHS. The sample includes 48 months on each side of the donut-hole in panel (I) and 84 months on each side of the donut-hole in panel (II). The samples in panel (A) are restricted to individuals aged 14 and above, while the samples in panel (B) are restricted to individuals aged 15 and above. The number of observations is 12,533 in all columns in panel (A) of part (I), while it is 11,055 in all columns in panel (B) of part (I). In part (II), the number of observations is 21,769 in all columns in panel (A) and 19,221 in all columns in panel (B). The policy dummy is one when year of birth is greater than 1987. The estimates in each column come from a separate logistic regression of marriage hazard status in panel (A) and of birth hazard status in panel (B) on the interactions of the policy variable with each age dummy and the interactions of the specified time trends in month and year of birth with each age dummy. Odds ratios and their standard errors are given as estimated parameters. The standard errors are clustered at the month-and-year-of-birth level. Statistical significance is $* * *$ at 1 percent level, ${ }^{* *}$ at 5 percent level, $*$ at 10 percent level. 
Table B3: Policy Effect on Marriage Hazard Rate and First-Birth Hazard Rate by Age, Odds Ratios - Month-Year of Birth as Running Variable, 10-Year Intervals around the Donut-hole

\begin{tabular}{|c|c|c|c|c|c|c|c|c|c|}
\hline \multirow[b]{2}{*}{$\begin{array}{l}\text { Degree of } \\
\text { Polynomial } \\
\end{array}$} & \multicolumn{6}{|c|}{ Single Time Trend } & \multicolumn{3}{|c|}{ Split Time Trends } \\
\hline & $\begin{array}{c}\text { One } \\
(1)\end{array}$ & $\begin{array}{l}\text { Two } \\
(2)\end{array}$ & $\begin{array}{c}\text { Three } \\
(3)\end{array}$ & $\begin{array}{c}\text { Four } \\
(4)\end{array}$ & $\begin{array}{c}\text { Five } \\
(5)\end{array}$ & $\begin{array}{l}\text { Six } \\
(6) \\
\end{array}$ & $\begin{array}{l}\text { One } \\
(7)\end{array}$ & $\begin{array}{c}\text { Two } \\
(8) \\
\end{array}$ & $\begin{array}{c}\text { Three } \\
(9)\end{array}$ \\
\hline \multicolumn{10}{|c|}{ A) Dependent Variable: Marriage status conditional on not being married until that age } \\
\hline Age $=14$ & $\begin{array}{c}0.490 \\
(0.327)\end{array}$ & $\begin{array}{c}0.671 \\
(0.444)\end{array}$ & $\begin{array}{c}0.577 \\
(0.558)\end{array}$ & $\begin{array}{c}0.250 \\
(0.294)\end{array}$ & $\begin{array}{c}0.411 \\
(0.633)\end{array}$ & $\begin{array}{c}0.214 \\
(0.386)\end{array}$ & $\begin{array}{c}0.600 \\
(0.385)\end{array}$ & $\begin{array}{c}0.166 \\
(0.256)\end{array}$ & $\begin{array}{c}0.267 \\
(0.645)\end{array}$ \\
\hline Age $=15$ & $\begin{array}{c}0.367 * * * \\
(0.135)\end{array}$ & $\begin{array}{c}0.450 * * \\
(0.179)\end{array}$ & $\begin{array}{c}0.352 * * \\
(0.174)\end{array}$ & $\begin{array}{c}0.232 * * \\
(0.153)\end{array}$ & $\begin{array}{c}0.157 * * \\
(0.116)\end{array}$ & $\begin{array}{c}0.135^{* *} \\
(0.120)\end{array}$ & $\begin{array}{c}0.428 * * \\
(0.172)\end{array}$ & $\begin{array}{c}0.162 * * \\
(0.136)\end{array}$ & $\begin{array}{c}0.060 * \\
(0.091)\end{array}$ \\
\hline Age $=16$ & $\begin{array}{c}1.125 \\
(0.296)\end{array}$ & $\begin{array}{c}1.202 \\
(0.304)\end{array}$ & $\begin{array}{c}0.557 \\
(0.212)\end{array}$ & $\begin{array}{c}0.397 * * \\
(0.168)\end{array}$ & $\begin{array}{c}0.715 \\
(0.436)\end{array}$ & $\begin{array}{c}0.867 \\
(0.504)\end{array}$ & $\begin{array}{c}1.167 \\
(0.295)\end{array}$ & $\begin{array}{c}0.316^{* *} \\
(0.170)\end{array}$ & $\begin{array}{c}1.361 \\
(1.275)\end{array}$ \\
\hline Age $=17$ & $\begin{array}{c}2.607 * * * \\
(0.628)\end{array}$ & $\begin{array}{c}2.827 * * * \\
(0.643)\end{array}$ & $\begin{array}{c}2.980 * * * \\
(1.029)\end{array}$ & $\begin{array}{c}2.294 * * \\
(0.848)\end{array}$ & $\begin{array}{c}4.316^{* * *} \\
(2.159)\end{array}$ & $\begin{array}{c}4.438 * * * \\
(2.261)\end{array}$ & $\begin{array}{c}2.674 * * * \\
(0.614)\end{array}$ & $\begin{array}{c}2.810 * * \\
(1.343)\end{array}$ & $\begin{array}{c}10.713 * * * \\
(9.700)\end{array}$ \\
\hline Age $=18$ & $\begin{array}{c}1.320 \\
(0.282)\end{array}$ & $\begin{array}{c}1.428 \\
(0.327)\end{array}$ & $\begin{array}{c}1.023 \\
(0.309)\end{array}$ & $\begin{array}{c}0.833 \\
(0.298)\end{array}$ & $\begin{array}{c}0.807 \\
(0.326)\end{array}$ & $\begin{array}{c}1.247 \\
(0.644)\end{array}$ & $\begin{array}{c}1.402 \\
(0.309)\end{array}$ & $\begin{array}{c}0.770 \\
(0.315)\end{array}$ & $\begin{array}{c}0.715 \\
(0.524)\end{array}$ \\
\hline Age $=19$ & $\begin{array}{r}1.072 \\
(0.273) \\
\end{array}$ & $\begin{array}{r}1.324 \\
(0.369) \\
\end{array}$ & $\begin{array}{c}0.858 \\
(0.304) \\
\end{array}$ & $\begin{array}{r}0.649 \\
(0.321) \\
\end{array}$ & $\begin{array}{c}0.995 \\
(0.534) \\
\end{array}$ & $\begin{array}{r}2.027 \\
(1.538) \\
\end{array}$ & $\begin{array}{c}1.272 \\
(0.336) \\
\end{array}$ & $\begin{array}{c}0.542 \\
(0.340) \\
\end{array}$ & $\begin{array}{c}7.460 * \\
(7.687) \\
\end{array}$ \\
\hline \multicolumn{10}{|c|}{ B) Dependent Variable: Birth status conditional on not giving birth until that age } \\
\hline Age $=15$ & $\begin{array}{c}0.605 \\
(0.368)\end{array}$ & $\begin{array}{c}0.485 \\
(0.294)\end{array}$ & $\begin{array}{c}0.178 * * \\
(0.156)\end{array}$ & $\begin{array}{c}0.070 * * \\
(0.074)\end{array}$ & $\begin{array}{c}0.218 \\
(0.321)\end{array}$ & $\begin{array}{l}0.038^{*} \\
(0.070)\end{array}$ & $\begin{array}{c}0.459 \\
(0.290)\end{array}$ & $\begin{array}{c}0.013 * * \\
(0.023)\end{array}$ & $\begin{array}{c}0.011 \\
(0.032)\end{array}$ \\
\hline Age $=16$ & $\begin{array}{c}0.553 \\
(0.256)\end{array}$ & $\begin{array}{c}0.689 \\
(0.339)\end{array}$ & $\begin{array}{c}0.543 \\
(0.325)\end{array}$ & $\begin{array}{l}0.236^{*} \\
(0.187)\end{array}$ & $\begin{array}{l}0.205^{*} \\
(0.177)\end{array}$ & $\begin{array}{l}0.173^{*} \\
(0.161)\end{array}$ & $\begin{array}{c}0.623 \\
(0.313)\end{array}$ & $\begin{array}{c}0.128 * * \\
(0.132)\end{array}$ & $\begin{array}{c}0.027 * * \\
(0.045)\end{array}$ \\
\hline Age $=17$ & $\begin{array}{c}0.876 \\
(0.282)\end{array}$ & $\begin{array}{c}0.847 \\
(0.295)\end{array}$ & $\begin{array}{c}0.377 * * \\
(0.164)\end{array}$ & $\begin{array}{c}0.192 * * * \\
(0.109)\end{array}$ & $\begin{array}{c}0.221 * * \\
(0.145)\end{array}$ & $\begin{array}{c}0.244 * * \\
(0.174)\end{array}$ & $\begin{array}{c}0.830 \\
(0.288)\end{array}$ & $\begin{array}{c}0.110 * * * \\
(0.078)\end{array}$ & $\begin{array}{c}0.095 * * \\
(0.110)\end{array}$ \\
\hline Age $=18$ & $\begin{array}{l}1.798 * * \\
(0.488)\end{array}$ & $\begin{array}{c}2.354 * * * \\
(0.584)\end{array}$ & $\begin{array}{c}2.245^{* *} \\
(0.858)\end{array}$ & $\begin{array}{c}1.669 \\
(0.712)\end{array}$ & $\begin{array}{l}2.679 * \\
(1.524)\end{array}$ & $\begin{array}{c}4.553 * * \\
(2.851)\end{array}$ & $\begin{array}{c}2.105 * * * \\
(0.510)\end{array}$ & $\begin{array}{c}1.843 \\
(0.962)\end{array}$ & $\begin{array}{c}8.209 * * \\
(7.646)\end{array}$ \\
\hline Age $=19$ & $\begin{array}{c}1.471 \\
(0.350) \\
\end{array}$ & $\begin{array}{c}1.739 * * \\
(0.427)\end{array}$ & $\begin{array}{l}1.905 * \\
(0.652)\end{array}$ & $\begin{array}{c}1.691 \\
(0.735) \\
\end{array}$ & $\begin{array}{c}1.383 \\
(0.657) \\
\end{array}$ & $\begin{array}{c}2.436 \\
(1.548)\end{array}$ & $\begin{array}{c}1.662 * * \\
(0.390)\end{array}$ & $\begin{array}{c}2.005 \\
(0.957) \\
\end{array}$ & $\begin{array}{c}1.357 \\
(1.174)\end{array}$ \\
\hline
\end{tabular}

Notes: The data come from the 2013 DHS. The sample includes 10 years on each side of the donut-hole. The samples in panel (A) are restricted to individuals aged 14 and above, while the samples in panel (B) are restricted to individuals aged 15 and above. The number of observations is 30,151 in all columns in panel (A), while it is 26,220 in all columns in panel (B). The policy dummy is one when year of birth is greater than 1987. The estimates in each column come from a separate logistic regression of marriage hazard status in panel (A) and of birth hazard status in panel (B) on the interactions of the policy variable with each age dummy and the interactions of the specified time trends in month and year of birth with each age dummy. Odds ratios and their standard errors are given as estimated parameters. The standard errors are clustered at the month-and-year-of-birth level. Statistical significance is *** at 1 percent level, ** at 5 percent level, * at 10 percent level. 
Table B4: Policy Effect on Marriage and First-Birth Hazard Rates by Age, Odds Ratios Sample (A) and Year of Birth as Running Variable

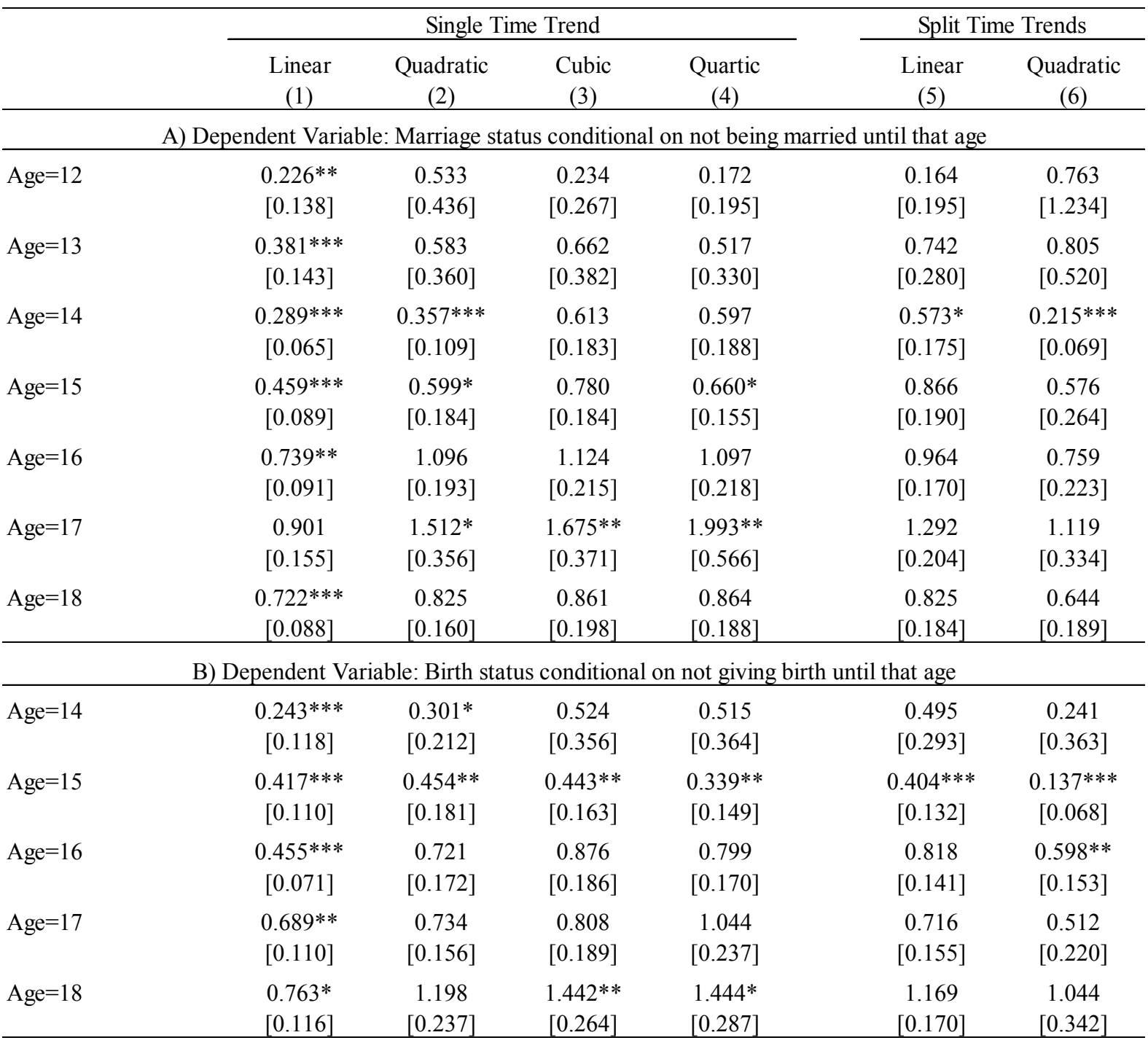

Notes: The sample includes observations from both 2008 and 2013 DHS. It includes 1959 to 1993 birth cohorts in the 2008 survey and 1964 to 1998 birth cohorts in the 2013 survey. 1986 and 1987 birth cohorts are excluded in all cases. The data are in person-age format. In panel (A), individuals who are never married by age 12 enter the risk set at this age and stay in the risk set until they get married or until the last age they are observed in the sample. Similarly, in panel (B), individuals who have never given birth by age 14 enter the risk set at this age and stay in the risk set until they give birth or until the last age they are observed in the sample. The number of observations is 141,622 in panel (A) and 108,361 in panel (B). The policy dummy is one when year of birth is greater than 1987. The estimates in each column come from a separate logistic regression of marriage hazard status in panel (A) and first-birth hazard status in panel (B) on the interactions of the policy dummy with each age dummy as well as the interactions of the specified time trends with each age dummy. Odds ratios and their standard errors are given as estimated parameters. In the first 4 columns, single time trends up to quartic polynomials are fitted, whereas separate polynomials are fitted on either side of the discontinuity in columns (5) and (6). The standard errors are clustered at the year-of-birth level. Statistical significance is *** at 1 percent level, ** at 5 percent level, * at 10 percent level. 


\section{APPENDIX C: FURTHER ROBUSTNESS CHECKS}

The main text already includes two sets of robustness checks; we check the assumption that the relationship between the outcome variables and the running variable is continuous except at the cutoff, and we examine any potential effects of the policy on baseline covariates at the cut-off. This section conducts further robustness checks. First, we conduct falsification checks in which we examine the effects of policies that did not happen. Second, we check the robustness of our findings to the inclusion of other control variables. Third, we inspect the sensitivity of our findings to the length of the donut-hole around the cut-off. Finally, we examine the potential effects of the change in Civil Code in 2002.

Our first robustness check concerns testing the effects of education policies that did not take place. For this purpose, we define counterfactual policies by sliding the timing of the policy over time and estimate equation (4) using these counterfactual policy dummies. The actual timing of the policy is slid incrementally by 4 months up to a maximum of 20 months in each direction. Tables $\mathrm{C} 1$ and $\mathrm{C} 2$ present the results for the policy effect on marriage and first-birth by age and on marriage and firstbirth hazard rates at each age, respectively. These results are given for three cases: sample (B1) with split linear polynomials, sample (B2) with split quadratic polynomials, and sample (B3) with single quartic polynomials. The results in Table $\mathrm{C} 1$ (Table $\mathrm{C} 2$ ) include the ages for which there is evidence of a policy effect in Tables 2 and 3 (Tables B1 and B2 in Appendix B).

In both Tables $\mathrm{C} 1$ and $\mathrm{C} 2$, the policy effect gradually diminishes and the odds ratios gradually approach one, as expected, as we move away from the discontinuity. In fact, when we move the actual timing of the policy by more than a year on either side, there is no evidence of a policy effect in either table. At the same time, the policy effect persists in some cases when the threshold of the counterfactual policy is not very distant from that of the actual. This occurs because, with a small 
perturbation, most of the points on the right-hand side are still treated and most on the left untreated. A similar analysis is conducted with sample (A), where counterfactuals are generated by sliding the timing of the policy by a year incrementally. The results, given in Table C3, confirm the above findings.

In the second robustness check, we check the sensitivity of our findings to the inclusion of other control variables when the running variable is month-year of birth. First, we add month of birth dummies to our level regressions (given in Table C4 for samples (B1) and (B2) and in Table C5 for sample (B3)) and to our timing regressions (given in Table C6 for samples (B1) and (B2) and Table C7 for sample (B3)). Second, in addition to the month of birth dummies, we also add dummies for place of birth in terms of 12 NUTS-1 regions and rural/urban status to our level regressions (given in Table C8 for samples (B1) and (B2) and in Table C9 for sample (B3)) and to our timing regressions (given in Table C10 for samples (B1) and (B2) and Table C11 for sample (B3)). The inclusion of these additional control variables makes very little difference; not only do the qualitative findings hold but the magnitudes of the coefficients are also very similar.

The third robustness check examines the sensitivity of our main findings on the policy effect on the probabilities of ever being married and ever giving birth by age to the width of the donut-hole we take. Table C12 presents the estimates with alternative donut-holes that increases incrementally by 3 months from 6 months to 18 months on each side of the cut-off. The coefficient mangitudes for the statistically significant estimates — for ages 15 and 16 in the marriage analysis and for ages 16 and 17 in the birth analysis - are very similar when even wider donut-holes (15-month and 18-month intervals) are taken; however, statistical significance is somewhat lower due to smaller sample sizes. When 9-month intervals on each side are taken, again the coefficient estimates are very similar; and the same patterns of statistical significance hold. However, when 6-month intervals are taken, the 
magnitudes of policy effects diminish (odds ratios become closer to one) and statistical significance is lost — as expected — as fuzziness around the cut-off starts to overwhelm the policy effect. That both the statistical significance and the magnitude of our estimates of the policy effect are very robust with donut-holes ranging from 9 to 18 months on each side is very assuring.

Finally, we examine the potential effects of the 2002 change in Civil Code. Prior to 2002, the Civil Code stipulated age 15 as the minimum age for marriage for women in Turkey. With a law that went into effect on 1 January 2002, the legal age of marriage was increased to 17 years for women. ${ }^{22}$ Nonetheless, as explained in Section 3.1, a non-trivial percentage of women marry before the legal age through religious ceremonies. For instance, according to the 2008 TDHS, 3.84 percent of 16year-old women were already married. Moreover, a significant percentage of women remained only with a religious marriage even after reaching the legal age of marriage. The percentage of 15- to 19year-old married women who had only a religious marriage ceremony was 33 percent in 1993, and dropped only to 29 percent by 2008 (TDHS data). Therefore, we might not expect the Civil Code to be binding on marriage decisions. Nonetheless, we conduct an empirical examination of this issue.

An individual born in December 1984 is not affected by the 2002 Civil Code because she was already 17 years old when the policy went into effect on January 1 of that year. Those born after January 1985 are affected, but the duration for which they are affected depends on their birth date. For instance, somebody born in January 1985 was affected only for a month because she was not allowed to get married in January 2002 but was able to marry in February 2002 (when she completed her $17^{\text {th }}$

\footnotetext{
${ }^{22}$ Under unusual circumstances such as pregnancy and with parental consent and a court decree, a 16-year-old is allowed to get married after the policy and a 14-year-old before the policy.
} 
year). In the same manner, somebody born in January 1986 was affected for a duration of 13 months, and somebody born in December 1986 was affected for a duration of 24 months.

We limit our analysis to individuals born before January 1987 to ensure that the individuals in our sample were not affected by the education policy. As explained above, the 1985 and 1986 birth cohorts were affected by the new Civil Code to varying degrees. Our sample also includes the 1983 and 1984 birth cohorts who were not affected by the Civil Code. We conduct a RDD analysis using this time window (1983-1986), month-year of birth as the running variable, and January 1985 as the cut-off. This time, however, the key variable of interest is not a dummy variable for the policy but a variable for the duration of exposure to the new policy in months (which varies from 1 to 24).

The estimation results, presented in Table C13, suggest that there might be small effects of the 2002 Civil Code on the marriage hazard rate at age 17. However, this result is not robust across specifications. While there is evidence of a positive policy effect on the marriage hazard rate at age 17 with single polynomials of various orders, there is no evidence with split polynomials. In addition, even when there is evidence of an effect, the magnitudes of the effects are tiny (the highest odds ratio is around 1.3) compared to the estimated effects of the education policy on the marriage hazard rate at age 17 given in Tables B2 and B3 of Appendix B (which are around 3-4). Furthermore, this effect might be partly capturing the effect of the education policy because the 1986 birth-cohort is partly affected. Hence, we conclude that there is either no or a small effect of the Civil Code on the marriage hazard rate at age 17, and even if such an effect exists, its magnitude is very small compared to the effect of the education policy. There is no evidence of an effect of the Civil Code on the marriage hazard rate at other ages. 
Table C1: Falsification Test of Level Effects of the Policy, Odds ratios

\begin{tabular}{|c|c|c|c|c|c|c|c|c|c|c|c|}
\hline & $(1)$ & $(2)$ & (3) & $(4)$ & $(5)$ & $(6)$ & $(7)$ & $(8)$ & $(9)$ & $(10)$ & $(11)$ \\
\hline \multicolumn{12}{|c|}{ I) 4-Year Intervals around the Bubble, Split Linear Polynomials } \\
\hline & \multicolumn{11}{|c|}{ Location of Chosen Discontinuity compared to the Actual Discontinuity (in months) } \\
\hline & -20 & -16 & -12 & -8 & -4 & 0 & +4 & +8 & +12 & +16 & +20 \\
\hline \multicolumn{12}{|c|}{ A) Dependent Variable: Ever Married } \\
\hline Age $=15$ & $\begin{array}{c}0.928 \\
{[0.542]}\end{array}$ & $\begin{array}{c}0.729 \\
{[0.464]}\end{array}$ & $\begin{array}{c}0.537 \\
{[0.403]}\end{array}$ & $\begin{array}{c}0.869 \\
{[0.613]}\end{array}$ & $\begin{array}{c}0.339^{*} \\
{[0.191]}\end{array}$ & $\begin{array}{l}0.285^{* *} \\
{[0.161]}\end{array}$ & $\begin{array}{c}0.489 \\
{[0.287]}\end{array}$ & $\begin{array}{c}0.608 \\
{[0.378]}\end{array}$ & $\begin{array}{c}0.674 \\
{[0.418]}\end{array}$ & $\begin{array}{c}1.590 \\
{[0.942]}\end{array}$ & $\begin{array}{r}1.079 \\
{[0.787]}\end{array}$ \\
\hline Age $=16$ & $\begin{array}{c}0.813 \\
{[0.305]}\end{array}$ & $\begin{array}{c}1.004 \\
{[0.367]}\end{array}$ & $\begin{array}{c}0.865 \\
{[0.309]}\end{array}$ & $\begin{array}{c}0.983 \\
{[0.361]}\end{array}$ & $\begin{array}{c}0.735 \\
{[0.260]}\end{array}$ & $\begin{array}{l}0.483 * * \\
{[0.167]}\end{array}$ & $\begin{array}{c}0.776 \\
{[0.302]}\end{array}$ & $\begin{array}{c}0.889 \\
{[0.368]}\end{array}$ & $\begin{array}{c}1.027 \\
{[0.437]}\end{array}$ & $\begin{array}{c}1.426 \\
{[0.594]}\end{array}$ & $\begin{array}{r}1.038 \\
{[0.490]}\end{array}$ \\
\hline \multicolumn{12}{|c|}{ B) Dependent Variable: Ever Given Birth } \\
\hline Age $=16$ & $\begin{array}{c}1.567 \\
{[1.163]}\end{array}$ & $\begin{array}{c}1.214 \\
{[0.984]}\end{array}$ & $\begin{array}{c}0.642 \\
{[0.582]}\end{array}$ & $\begin{array}{c}1.338 \\
{[1.160]}\end{array}$ & $\begin{array}{c}0.310 \\
{[0.231]}\end{array}$ & $\begin{array}{l}0.200^{* *} \\
{[0.147]}\end{array}$ & $\begin{array}{c}0.415 \\
{[0.292]}\end{array}$ & $\begin{array}{c}0.333 \\
{[0.251]}\end{array}$ & $\begin{array}{c}0.341 \\
{[0.275]}\end{array}$ & $\begin{array}{c}0.691 \\
{[0.549]}\end{array}$ & $\begin{array}{r}0.655 \\
{[0.495]}\end{array}$ \\
\hline Age $=17$ & $\begin{array}{c}0.601 \\
{[0.332]}\end{array}$ & $\begin{array}{c}0.621 \\
{[0.363]}\end{array}$ & $\begin{array}{c}0.545 \\
{[0.354]}\end{array}$ & $\begin{array}{c}0.764 \\
{[0.478]}\end{array}$ & $\begin{array}{c}0.371^{*} \\
{[0.219]}\end{array}$ & $\begin{array}{l}0.313^{* *} \\
{[0.143]}\end{array}$ & $\begin{array}{c}0.837 \\
{[0.363]}\end{array}$ & $\begin{array}{c}0.945 \\
{[0.452]}\end{array}$ & $\begin{array}{c}1.030 \\
{[0.501]}\end{array}$ & $\begin{array}{c}1.583 \\
{[0.815]}\end{array}$ & $\begin{array}{c}0.888 \\
{[0.572]}\end{array}$ \\
\hline
\end{tabular}

II) 7-Year Intervals around the Bubble, Split Quadratic Polynomials

Location of Chosen Discontinuity compared to the Actual Discontinuity (in months)

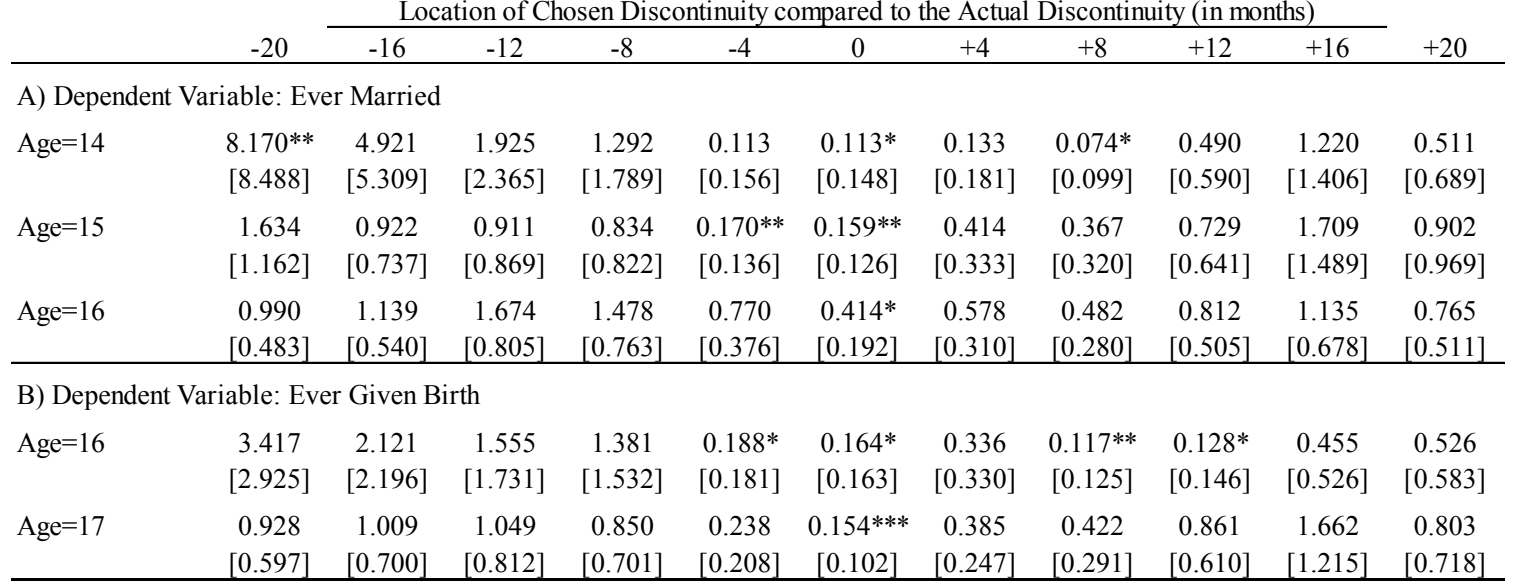

II) 7-Year Intervals around the Bubble, Single Quartic Polynomials

Location of Chosen Discontinuity compared to the Actual Discontinuity (in months)

\begin{tabular}{lccccccccccc}
\multicolumn{1}{c}{} & -20 & -16 & -12 & -8 & -4 & 0 & +4 & +8 & +12 & +16 & +20 \\
\hline \multirow{2}{*}{ A) Dependent Variable: Ever Married } & & & & & & & & & & \\
Age $=14$ & $4.018^{*}$ & 2.807 & 1.405 & 0.941 & 0.187 & $0.175^{*}$ & 0.195 & $0.148^{*}$ & 0.673 & 1.375 & 0.647 \\
& {$[3.373]$} & {$[2.483]$} & {$[1.448]$} & {$[1.119]$} & {$[0.198]$} & {$[0.183]$} & {$[0.214]$} & {$[0.160]$} & {$[0.661]$} & {$[1.286]$} & {$[0.705]$} \\
\multirow{2}{*}{ Age=15 } & 1.233 & 0.788 & 0.777 & 0.629 & $0.215^{* *}$ & $0.232^{* *}$ & 0.465 & 0.457 & 0.768 & 1.457 & 0.882 \\
& {$[0.743]$} & {$[0.537]$} & {$[0.602]$} & {$[0.517]$} & {$[0.130]$} & {$[0.145]$} & {$[0.297]$} & {$[0.309]$} & {$[0.537]$} & {$[0.995]$} & {$[0.774]$} \\
\multirow{2}{*}{ Age $=16$} & 0.927 & 1.011 & 1.323 & 1.113 & 0.687 & $0.442^{* *}$ & 0.582 & 0.534 & 0.819 & 1.062 & 0.813 \\
& {$[0.361]$} & {$[0.384]$} & {$[0.504]$} & {$[0.458]$} & {$[0.260]$} & {$[0.167]$} & {$[0.257]$} & {$[0.258]$} & {$[0.418]$} & {$[0.514]$} & {$[0.439]$} \\
\hline
\end{tabular}

B) Dependent Variable: Ever Given Birth

\begin{tabular}{cccccccccccc} 
Age $=16$ & 2.017 & 1.317 & 1.077 & 0.969 & $0.252^{*}$ & $0.241^{*}$ & 0.383 & $0.196^{* *}$ & $0.210^{*}$ & 0.593 & 0.621 \\
& {$[1.523]$} & {$[1.175]$} & {$[0.977]$} & {$[0.873]$} & {$[0.178]$} & {$[0.182]$} & {$[0.292]$} & {$[0.162]$} & {$[0.191]$} & {$[0.536]$} & {$[0.579]$} \\
Age $=17$ & 0.779 & 0.830 & 0.862 & 0.694 & $0.288^{* *}$ & $0.231 * * *$ & 0.462 & 0.511 & 0.911 & 1.496 & 0.893 \\
& {$[0.422]$} & {$[0.475]$} & {$[0.523]$} & {$[0.442]$} & {$[0.176]$} & {$[0.123]$} & {$[0.242]$} & {$[0.285]$} & {$[0.526]$} & {$[0.875]$} & {$[0.642]$} \\
\hline
\end{tabular}

Notes: In each column, a different cut-off value for month-year of birth is taken in defining the policy dummy -- as specified in the column heading. For instance, (-4) means that the policy dummy is one when month-year of birth is greater than the actual value of the cut-off (January 1, 1987) minus 4 months, which is September 1, 1987. In each column, a bubble of 12 months is taken on each side of the cut-off as it is done in the actual analysis. Each cell comes from a separate logistic regression of ever-married status at the specified age in panel (A) and of ever-given-birth status at the specified age in panel (B) on the policy variable and the time trends--which are specified in the headings. Odds ratios and their standard erros are given as the estimated coefficients. The standard errors are clustered at the month-year of birth level. Statistical significance is $* * *$ at 1 percent level, ** at 5 percent level, * at 10 percent level. 
Table C2: Falsification Test of Timing Effects of the Policy, Odds ratios

\begin{tabular}{|c|c|c|c|c|c|c|c|c|c|c|c|}
\hline & $(1)$ & $(2)$ & $(3)$ & $(4)$ & $(5)$ & $(6)$ & $(7)$ & $(8)$ & $(9)$ & $(10)$ & $(11)$ \\
\hline \multicolumn{12}{|c|}{ I) 4-Year Intervals around the Bubble, Split Linear Polynomials } \\
\hline & \multicolumn{11}{|c|}{ Location of Chosen Discontinuity compared to the Actual Discontinuity (in months) } \\
\hline & -20 & -16 & -12 & -8 & -4 & 0 & +4 & +8 & +12 & +16 & +20 \\
\hline \multicolumn{12}{|c|}{ A) Dependent Variable: Marriage status conditional on not being married until that age } \\
\hline Age $=15$ & $\begin{array}{c}0.352 \\
(0.307)\end{array}$ & $\begin{array}{c}0.328 \\
(0.274)\end{array}$ & $\begin{array}{c}0.331 \\
(0.331)\end{array}$ & $\begin{array}{c}0.650 \\
(0.571)\end{array}$ & $\begin{array}{l}0.313 * \\
(0.211)\end{array}$ & $\begin{array}{l}0.308^{*} \\
(0.220)\end{array}$ & $\begin{array}{c}0.583 \\
(0.468)\end{array}$ & $\begin{array}{c}0.719 \\
(0.595)\end{array}$ & $\begin{array}{c}0.772 \\
(0.622)\end{array}$ & $\begin{array}{c}1.879 \\
(1.489)\end{array}$ & $\begin{array}{c}1.672 \\
(1.669)\end{array}$ \\
\hline Age $=17$ & $\begin{array}{c}0.609 \\
(0.263) \\
\end{array}$ & $\begin{array}{c}1.163 \\
(0.487) \\
\end{array}$ & $\begin{array}{c}1.713 \\
(0.676) \\
\end{array}$ & $\begin{array}{l}2.695 * * \\
(1.069) \\
\end{array}$ & $\begin{array}{c}4.042^{* * *} \\
(1.585) \\
\end{array}$ & $\begin{array}{c}5.300^{* * *} \\
(2.275) \\
\end{array}$ & $\begin{array}{l}3.197 * * \\
(1.707) \\
\end{array}$ & $\begin{array}{c}4.545^{* * * *} \\
(2.583) \\
\end{array}$ & $\begin{array}{c}4.270^{* * *} \\
(2.200) \\
\end{array}$ & $\begin{array}{r}2.234 \\
(1.139) \\
\end{array}$ & $\begin{array}{r}0.867 \\
(0.414) \\
\end{array}$ \\
\hline \multicolumn{12}{|c|}{ B) Dependent Variable: Birth status conditional on not giving birth until that age } \\
\hline Age $=15$ & $\begin{array}{c}2.482 \\
(3.007)\end{array}$ & $\begin{array}{c}0.742 \\
(1.190)\end{array}$ & $\begin{array}{c}1.474 \\
(2.804)\end{array}$ & $\begin{array}{c}3.330 \\
(6.018)\end{array}$ & $\begin{array}{c}0.774 \\
(1.336)\end{array}$ & $\begin{array}{l}0.053^{*} \\
(0.081)\end{array}$ & $\begin{array}{c}0.078 \\
(0.121)\end{array}$ & $\begin{array}{c}0.143 \\
(0.183)\end{array}$ & $\begin{array}{c}0.175 \\
(0.219)\end{array}$ & $\begin{array}{c}0.587 \\
(0.676)\end{array}$ & $\begin{array}{c}0.537 \\
(0.714)\end{array}$ \\
\hline Age $=16$ & $\begin{array}{c}0.872 \\
(0.862)\end{array}$ & $\begin{array}{c}0.739 \\
(0.724)\end{array}$ & $\begin{array}{c}0.199 \\
(0.201)\end{array}$ & $\begin{array}{c}0.574 \\
(0.477)\end{array}$ & $\begin{array}{l}0.123^{* *} \\
(0.118)\end{array}$ & $\begin{array}{l}0.165^{*} \\
(0.155)\end{array}$ & $\begin{array}{c}0.385 \\
(0.348)\end{array}$ & $\begin{array}{c}0.552 \\
(0.494)\end{array}$ & $\begin{array}{c}0.517 \\
(0.507)\end{array}$ & $\begin{array}{c}1.057 \\
(1.104)\end{array}$ & $\begin{array}{c}1.082 \\
(1.014)\end{array}$ \\
\hline Age $=18$ & $\begin{array}{c}0.841 \\
(0.367)\end{array}$ & $\begin{array}{c}1.180 \\
(0.516)\end{array}$ & $\begin{array}{c}0.938 \\
(0.378)\end{array}$ & $\begin{array}{c}1.209 \\
(0.504)\end{array}$ & $\begin{array}{c}1.623 \\
(0.660)\end{array}$ & $\begin{array}{l}2.393^{*} \\
(1.109)\end{array}$ & $\begin{array}{l}2.587 * * \\
(1.165)\end{array}$ & $\begin{array}{c}3.230 * * * \\
(1.420)\end{array}$ & $\begin{array}{c}3.499^{* * *} \\
(1.632)\end{array}$ & $\begin{array}{l}2.427 * \\
(1.222)\end{array}$ & $\begin{array}{c}1.683 \\
(0.881)\end{array}$ \\
\hline
\end{tabular}

II) 7-Year Intervals around the Bubble, Split Quadratic Polynomials

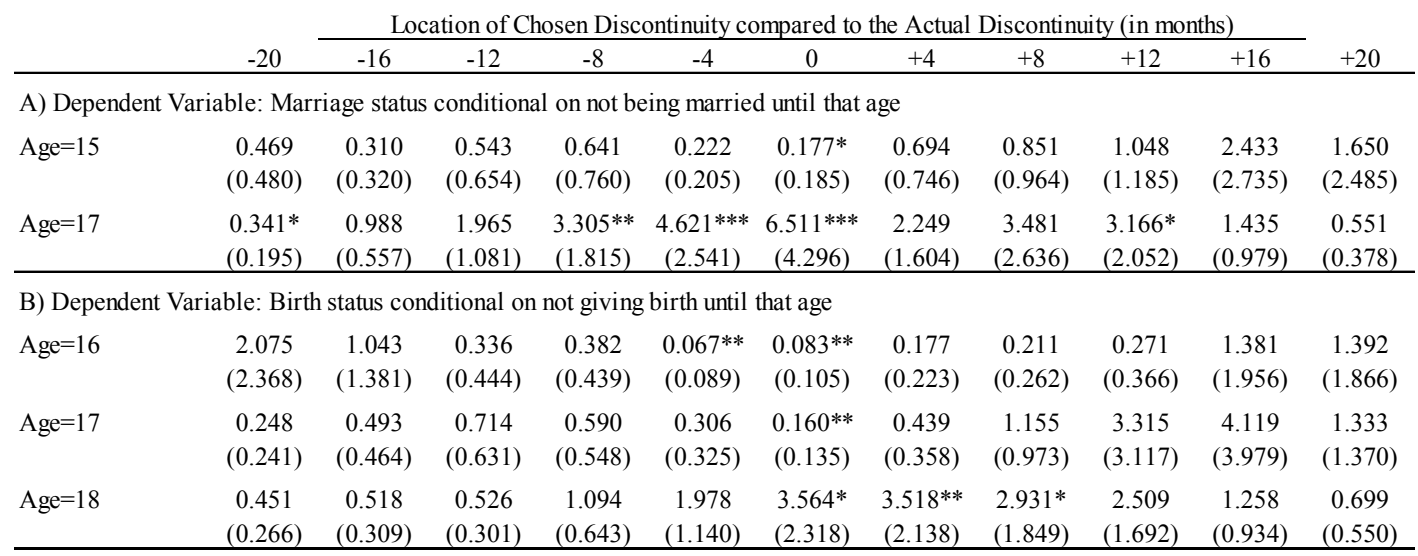

II) 7-Year Intervals around the Bubble, Single Quartic Polynomials

\begin{tabular}{|c|c|c|c|c|c|c|c|c|c|c|c|}
\hline & \multicolumn{11}{|c|}{ Location of Chosen Discontinuity compared to the Actual Discontinuity (in months) } \\
\hline & -20 & -16 & -12 & -8 & -4 & 0 & +4 & +8 & +12 & +16 & +20 \\
\hline \multicolumn{12}{|c|}{ A) Dependent Variable: Marriage status conditional on not being married until that age } \\
\hline Age $=15$ & $\begin{array}{c}0.497 \\
(0.420)\end{array}$ & $\begin{array}{c}0.336 \\
(0.293)\end{array}$ & $\begin{array}{c}0.517 \\
(0.499)\end{array}$ & $\begin{array}{c}0.486 \\
(0.472)\end{array}$ & $\begin{array}{c}0.232 * * \\
(0.167)\end{array}$ & $\begin{array}{c}0.237 * \\
(0.189)\end{array}$ & $\begin{array}{c}0.621 \\
(0.514)\end{array}$ & $\begin{array}{c}0.743 \\
(0.633)\end{array}$ & $\begin{array}{c}0.877 \\
(0.757)\end{array}$ & $\begin{array}{c}1.612 \\
(1.372)\end{array}$ & $\begin{array}{c}1.283 \\
(1.503)\end{array}$ \\
\hline Age $=17$ & $\begin{array}{c}0.481 \\
(0.220)\end{array}$ & $\begin{array}{c}1.047 \\
(0.478)\end{array}$ & $\begin{array}{c}1.751 \\
(0.770)\end{array}$ & $\begin{array}{l}2.524 * * \\
(1.091)\end{array}$ & $\begin{array}{c}3.174 * * * \\
(1.348)\end{array}$ & $\begin{array}{c}4.157 * * * \\
(1.974)\end{array}$ & $\begin{array}{c}2.255 \\
(1.194)\end{array}$ & $\begin{array}{l}3.304^{* *} \\
(1.859)\end{array}$ & $\begin{array}{l}3.229 * * \\
(1.647)\end{array}$ & $\begin{array}{c}1.828 \\
(0.983)\end{array}$ & $\begin{array}{c}0.913 \\
(0.513)\end{array}$ \\
\hline
\end{tabular}

B) Dependent Variable: Birth status conditional on not giving birth until that age

\begin{tabular}{ccccccccccccc} 
Age=16 & 1.310 & 0.710 & 0.320 & 0.357 & $0.143^{* *}$ & $0.191^{*}$ & 0.307 & 0.324 & 0.400 & 1.407 & 1.317 \\
& $(1.264)$ & $(0.748)$ & $(0.324)$ & $(0.316)$ & $(0.130)$ & $(0.177)$ & $(0.290)$ & $(0.315)$ & $(0.432)$ & $(1.551)$ & $(1.477)$ \\
Age=17 & 0.317 & 0.515 & 0.658 & 0.538 & 0.344 & $0.240^{* *}$ & 0.526 & 1.059 & 2.449 & 3.046 & 1.348 \\
& $(0.238)$ & $(0.373)$ & $(0.450)$ & $(0.380)$ & $(0.264)$ & $(0.161)$ & $(0.345)$ & $(0.717)$ & $(1.770)$ & $(2.276)$ & $(1.100)$ \\
Age=18 & 0.621 & 0.690 & 0.696 & 1.177 & 1.794 & $2.655^{*}$ & $2.855^{* *}$ & $2.502^{*}$ & 2.252 & 1.341 & 0.885 \\
& $(0.292)$ & $(0.327)$ & $(0.316)$ & $(0.545)$ & $(0.815)$ & $(1.325)$ & $(1.359)$ & $(1.221)$ & $(1.172)$ & $(0.766)$ & $(0.533)$ \\
\hline
\end{tabular}

Notes: In each column, a different cut-off value for month-year of birth is taken in defining the policy dummy -- as specified in the column heading. For instance, (-4) means that the policy dummy is one when month-year of birth is greater than the actual value of the cut-off (January 1, 1987) minus 4 months, which is September 1, 1987. In each column, a bubble of 12 months is taken on each side of the cut-off as it is done in the actual analysis. Each cell comes from a separate logistic regression of ever-married status at the specified age in panel (A) and of ever-given-birth status at the specified age in panel (B) on the interactions of the policy variable with each age dummy as well as the interactions of age dummies with the time trends--which are specified in the headings. The coefficients in each cell denote the policy effect for that age, calculated as the linear combination of the baseline policy coefficient and the policy coefficient for that specific age. Odds ratios and their standard erros are given as the estimated coefficients. The standard errors are clustered at the monthyear of birth level. Statistical significance is $* * *$ at 1 percent level, ${ }^{* *}$ at 5 percent level, ${ }^{*}$ at 10 percent level. 
Table C3: Falsification Test with Sample (A), Odds ratios

\begin{tabular}{|c|c|c|c|c|c|c|c|c|c|}
\hline \multicolumn{10}{|c|}{ A) Dependent Variable: Ever Married } \\
\hline & & \multicolumn{8}{|c|}{ Year of Discontinuity } \\
\hline & & $\begin{array}{c}1983 \\
(1)\end{array}$ & $\begin{array}{c}1984 \\
(2)\end{array}$ & $\begin{array}{c}1985 \\
(3)\end{array}$ & $\begin{array}{c}1986 \\
(4)\end{array}$ & $\begin{array}{c}\text { Actual } \\
(5)\end{array}$ & $\begin{array}{c}1988 \\
(6)\end{array}$ & $\begin{array}{c}1989 \\
(7)\end{array}$ & $\begin{array}{c}1990 \\
(8)\end{array}$ \\
\hline \multirow[t]{2}{*}{ Age $=14$} & & $\begin{array}{c}0.932 \\
{[0.283]}\end{array}$ & $\begin{array}{c}0.986 \\
{[0.309]}\end{array}$ & $\begin{array}{c}0.998 \\
{[0.386]}\end{array}$ & $\begin{array}{l}0.624^{*} \\
{[0.151]}\end{array}$ & $\begin{array}{c}0.546^{* *} \\
{[0.130]}\end{array}$ & $\begin{array}{c}0.553^{* *} \\
{[0.138]}\end{array}$ & $\begin{array}{c}0.750 \\
{[0.274]}\end{array}$ & $\begin{array}{c}0.853 \\
{[0.447]}\end{array}$ \\
\hline & No obs. & 20,588 & 20,616 & 20,625 & 20,527 & 20,552 & 20,542 & 20,502 & 20,470 \\
\hline \multirow[t]{2}{*}{ Age $=15$} & & $\begin{array}{c}1.023 \\
{[0.257]}\end{array}$ & $\begin{array}{c}0.886 \\
{[0.221]}\end{array}$ & $\begin{array}{c}0.751 \\
{[0.133]}\end{array}$ & $\begin{array}{c}0.649^{* * *} \\
{[0.094]}\end{array}$ & $\begin{array}{c}0.611 * * * \\
{[0.098]}\end{array}$ & $\begin{array}{l}0.656^{*} \\
{[0.152]}\end{array}$ & $\begin{array}{c}0.945 \\
{[0.203]}\end{array}$ & $\begin{array}{c}0.975 \\
{[0.285]}\end{array}$ \\
\hline & No obs. & 20,588 & 20,616 & 20,625 & 20,527 & 20,552 & 20,542 & 20,502 & 20,470 \\
\hline \multirow[t]{2}{*}{ Age $=16$} & & $\begin{array}{c}1.094 \\
{[0.165]}\end{array}$ & $\begin{array}{c}0.957 \\
{[0.124]}\end{array}$ & $\begin{array}{c}0.888 \\
{[0.100]}\end{array}$ & $\begin{array}{c}0.838 \\
{[0.111]}\end{array}$ & $\begin{array}{l}0.777^{* *} \\
{[0.086]}\end{array}$ & $\begin{array}{c}0.868 \\
{[0.137]}\end{array}$ & $\begin{array}{c}0.933 \\
{[0.169]}\end{array}$ & $\begin{array}{c}0.891 \\
{[0.199]}\end{array}$ \\
\hline & No obs. & 19,768 & 19,796 & 19,805 & 19,707 & 19,732 & 19,722 & 19,682 & 19,650 \\
\hline \multicolumn{10}{|c|}{ B) Dependent Variable: Ever Given Birth } \\
\hline & & \multicolumn{8}{|c|}{ Year of Discontinuity } \\
\hline & & $\begin{array}{c}1983 \\
(1)\end{array}$ & $\begin{array}{c}1984 \\
(2)\end{array}$ & $\begin{array}{c}1985 \\
(3)\end{array}$ & $\begin{array}{c}1986 \\
(4) \\
\end{array}$ & $\begin{array}{c}\text { Actual } \\
(5)\end{array}$ & $\begin{array}{c}1988 \\
(6) \\
\end{array}$ & $\begin{array}{c}1989 \\
(7) \\
\end{array}$ & $\begin{array}{c}1990 \\
(8)\end{array}$ \\
\hline \multirow[t]{2}{*}{ Age $=15$} & & $\begin{array}{c}0.771 \\
{[0.254]}\end{array}$ & $\begin{array}{c}0.764 \\
{[0.297]}\end{array}$ & $\begin{array}{c}0.923 \\
{[0.559]}\end{array}$ & $\begin{array}{c}0.526 \\
{[0.225]}\end{array}$ & $\begin{array}{c}0.411^{* *} \\
{[0.153]}\end{array}$ & $\begin{array}{c}0.350 * * * \\
{[0.139]}\end{array}$ & $\begin{array}{c}0.624 \\
{[0.244]}\end{array}$ & $\begin{array}{c}0.879 \\
{[0.516]}\end{array}$ \\
\hline & No obs. & 20,588 & 20,616 & 20,625 & 20,527 & 20,552 & 20,542 & 20,502 & 20,470 \\
\hline \multirow[t]{2}{*}{ Age $=16$} & & $\begin{array}{c}1.153 \\
{[0.294]}\end{array}$ & $\begin{array}{c}1.185 \\
{[0.297]}\end{array}$ & $\begin{array}{c}1.004 \\
{[0.267]}\end{array}$ & $\begin{array}{c}0.731 * * \\
{[0.107]}\end{array}$ & $\begin{array}{c}0.610^{* * *} \\
{[0.114]}\end{array}$ & $\begin{array}{c}0.568 * * * \\
{[0.117]}\end{array}$ & $\begin{array}{c}0.822 \\
{[0.185]}\end{array}$ & $\begin{array}{c}0.929 \\
{[0.265]}\end{array}$ \\
\hline & No obs. & 19,768 & 19,796 & 19,805 & 19,707 & 19,732 & 19,722 & 19,682 & 19,650 \\
\hline \multirow[t]{2}{*}{ Age $=17$} & & $\begin{array}{c}1.067 \\
{[0.181]}\end{array}$ & $\begin{array}{c}1.018 \\
{[0.162]}\end{array}$ & $\begin{array}{c}0.934 \\
{[0.119]}\end{array}$ & $\begin{array}{c}0.875 \\
{[0.116]}\end{array}$ & $\begin{array}{l}0.773^{*} \\
{[0.112]}\end{array}$ & $\begin{array}{c}0.893 \\
{[0.163]}\end{array}$ & $\begin{array}{c}0.969 \\
{[0.270]}\end{array}$ & $\begin{array}{c}0.915 \\
{[0.431]}\end{array}$ \\
\hline & No obs. & 18,909 & 18,937 & 18,946 & 18,848 & 18,873 & 18,863 & 18,823 & 18,791 \\
\hline
\end{tabular}

Notes: In each column, a separate cut-off value of year of birth is taken in defining the policy dummy -- as specified in the column heading. For instance, in column (1), the policy dummy is one when year of birth is equal to 1983 or above, and zero otherwise. In each column, two birth cohorts around the cut-off are dropped; for instance, the 1982 and 1983 birth cohorts are dropped in column (1). Each cell comes from a separate logistic regression of ever-married status at the specified age in panel (A) and of ever-givenbirth status at the specified age in panel (B) on the policy variable and the time trends--which are single time trends in the form of quartic polynomials. Odds ratios and their standard erros are given as the estimated coefficients. The standard errors are clustered at the year-of-birth level. Statistical significance is $* * *$ at 1 percent level, $* *$ at 5 percent level, ${ }^{*}$ at 10 percent level. 
Table C4: Policy Effect on Marriage and First-Birth by Age with Dummies for Month of

Birth - 4-Year and 7-Year Intervals around the Donut-hole (Samples B1 and B2), Odds ratios

\begin{tabular}{|c|c|c|c|c|c|c|c|c|c|}
\hline \multirow[b]{3}{*}{$\begin{array}{l}\text { Degree of } \\
\text { Polynomial } \\
\end{array}$} & \multicolumn{3}{|c|}{ I) 4-Year Intervals } & \multicolumn{6}{|c|}{ II) 7-Year Intervals } \\
\hline & \multicolumn{2}{|c|}{$\begin{array}{c}\text { Single Time } \\
\text { Trend }\end{array}$} & \multirow{2}{*}{$\begin{array}{c}\begin{array}{c}\text { Split } \\
\text { Trends }\end{array} \\
\text { One } \\
(3)\end{array}$} & \multicolumn{4}{|c|}{ Single Time Trend } & \multicolumn{2}{|c|}{ Split Time Trends } \\
\hline & $\begin{array}{l}\text { One } \\
(1)\end{array}$ & $\begin{array}{c}\text { Two } \\
(2)\end{array}$ & & $\begin{array}{l}\text { One } \\
(4)\end{array}$ & $\begin{array}{c}\text { Two } \\
(5)\end{array}$ & $\begin{array}{c}\text { Three } \\
(6) \\
\end{array}$ & $\begin{array}{c}\text { Four } \\
(7)\end{array}$ & $\begin{array}{c}\text { One } \\
(8) \\
\end{array}$ & $\begin{array}{c}\text { Two } \\
(9) \\
\end{array}$ \\
\hline \multicolumn{10}{|c|}{ A) Dependent Variable: Ever Married } \\
\hline Age $=13$ & $\begin{array}{c}0.825 \\
{[0.793]}\end{array}$ & $\begin{array}{c}0.746 \\
{[0.764]}\end{array}$ & $\begin{array}{c}0.699 \\
{[0.750]}\end{array}$ & $\begin{array}{c}1.175 \\
{[0.878]}\end{array}$ & $\begin{array}{c}1.416 \\
{[1.003]}\end{array}$ & $\begin{array}{c}0.989 \\
{[1.084]}\end{array}$ & $\begin{array}{c}0.424 \\
{[0.575]}\end{array}$ & $\begin{array}{c}1.309 \\
{[0.933]}\end{array}$ & $\begin{array}{c}0.244 \\
{[0.480]}\end{array}$ \\
\hline Age $=14$ & $\begin{array}{c}0.378 \\
{[0.323]}\end{array}$ & $\begin{array}{c}0.319 \\
{[0.293]}\end{array}$ & $\begin{array}{c}0.309 \\
{[0.290]}\end{array}$ & $\begin{array}{c}0.861 \\
{[0.512]}\end{array}$ & $\begin{array}{c}0.850 \\
{[0.520]}\end{array}$ & $\begin{array}{c}0.478 \\
{[0.411]}\end{array}$ & $\begin{array}{c}0.200 \\
{[0.214]}\end{array}$ & $\begin{array}{c}0.814 \\
{[0.501]}\end{array}$ & $\begin{array}{c}0.124 \\
{[0.172]}\end{array}$ \\
\hline Age $=15$ & $\begin{array}{l}0.330^{* *} \\
{[0.164]}\end{array}$ & $\begin{array}{c}0.313^{* *} \\
{[0.169]}\end{array}$ & $\begin{array}{l}0.293 * * \\
{[0.160]}\end{array}$ & $\begin{array}{l}0.472^{* *} \\
{[0.168]}\end{array}$ & $\begin{array}{c}0.429^{* *} \\
{[0.171]}\end{array}$ & $\begin{array}{l}0.328^{*} \\
{[0.191]}\end{array}$ & $\begin{array}{l}0.248^{* *} \\
{[0.158]}\end{array}$ & $\begin{array}{l}0.416 * * \\
{[0.169]}\end{array}$ & $\begin{array}{l}0.171 * * \\
{[0.139]}\end{array}$ \\
\hline Age $=16$ & $\begin{array}{l}0.553^{*} \\
{[0.169]}\end{array}$ & $\begin{array}{l}0.518^{* *} \\
{[0.169]}\end{array}$ & $\begin{array}{l}0.507 * * \\
{[0.163]}\end{array}$ & $\begin{array}{l}0.559^{* *} \\
{[0.127]}\end{array}$ & $\begin{array}{c}0.517 * * * \\
{[0.124]}\end{array}$ & $\begin{array}{c}0.548^{*} \\
{[0.194]}\end{array}$ & $\begin{array}{l}0.466^{* *} \\
{[0.165]}\end{array}$ & $\begin{array}{c}0.508^{* * *} \\
{[0.123]}\end{array}$ & $\begin{array}{l}0.445^{*} \\
{[0.190]}\end{array}$ \\
\hline Age $=17$ & $\begin{array}{c}1.416 \\
{[0.420]}\end{array}$ & $\begin{array}{c}1.364 \\
{[0.401]}\end{array}$ & $\begin{array}{c}1.370 \\
{[0.401]}\end{array}$ & $\begin{array}{c}1.058 \\
{[0.203]}\end{array}$ & $\begin{array}{c}1.040 \\
{[0.197]}\end{array}$ & $\begin{array}{c}1.297 \\
{[0.419]}\end{array}$ & $\begin{array}{c}1.194 \\
{[0.383]}\end{array}$ & $\begin{array}{c}1.034 \\
{[0.195]}\end{array}$ & $\begin{array}{c}1.344 \\
{[0.559]}\end{array}$ \\
\hline Age $=18$ & $\begin{array}{c}1.134 \\
{[0.277]}\end{array}$ & $\begin{array}{c}1.123 \\
{[0.272]}\end{array}$ & $\begin{array}{c}1.125 \\
{[0.270]}\end{array}$ & $\begin{array}{c}1.085 \\
{[0.174]}\end{array}$ & $\begin{array}{l}1.075 \\
{[0.171]}\end{array}$ & $\begin{array}{c}1.246 \\
{[0.329]}\end{array}$ & $\begin{array}{c}1.221 \\
{[0.319]}\end{array}$ & $\begin{array}{l}1.075 \\
{[0.170]}\end{array}$ & $\begin{array}{c}1.253 \\
{[0.412]}\end{array}$ \\
\hline Age $=19$ & $\begin{array}{c}1.207 \\
{[0.296]}\end{array}$ & $\begin{array}{c}1.197 \\
{[0.292]}\end{array}$ & $\begin{array}{c}1.198 \\
{[0.290]}\end{array}$ & $\begin{array}{c}1.154 \\
{[0.183]}\end{array}$ & $\begin{array}{c}1.156 \\
{[0.184]}\end{array}$ & $\begin{array}{c}1.044 \\
{[0.273]}\end{array}$ & $\begin{array}{c}1.009 \\
{[0.262]}\end{array}$ & $\begin{array}{c}1.152 \\
{[0.182]}\end{array}$ & $\begin{array}{c}1.022 \\
{[0.337]}\end{array}$ \\
\hline \multicolumn{10}{|c|}{ B) Dependent Variable: Ever Given Birth } \\
\hline Age $=15$ & $\begin{array}{c}0.385 \\
{[0.397]}\end{array}$ & $\begin{array}{c}0.254 \\
{[0.336]}\end{array}$ & $\begin{array}{c}0.297 \\
{[0.378]}\end{array}$ & $\begin{array}{c}0.578 \\
{[0.332]}\end{array}$ & $\begin{array}{c}0.362 \\
{[0.253]}\end{array}$ & $\begin{array}{c}0.628 \\
{[0.713]}\end{array}$ & $\begin{array}{c}0.368 \\
{[0.515]}\end{array}$ & $\begin{array}{c}0.341 \\
{[0.255]}\end{array}$ & $\begin{array}{c}0.464 \\
{[0.802]}\end{array}$ \\
\hline Age $=16$ & $\begin{array}{l}0.335^{* *} \\
{[0.183]}\end{array}$ & $\begin{array}{c}0.223 * * \\
{[0.146]}\end{array}$ & $\begin{array}{l}0.212^{* *} \\
{[0.143]}\end{array}$ & $\begin{array}{c}0.561 \\
{[0.217]}\end{array}$ & $\begin{array}{l}0.423 * \\
{[0.191]}\end{array}$ & $\begin{array}{c}0.382 \\
{[0.244]}\end{array}$ & $\begin{array}{l}0.257^{*} \\
{[0.181]}\end{array}$ & $\begin{array}{l}0.402 * \\
{[0.190]}\end{array}$ & $\begin{array}{l}0.171^{*} \\
{[0.162]}\end{array}$ \\
\hline Age $=17$ & $\begin{array}{l}0.414^{* *} \\
{[0.159]}\end{array}$ & $\begin{array}{c}0.326 * * * \\
{[0.138]}\end{array}$ & $\begin{array}{c}0.311^{* * *} \\
{[0.133]}\end{array}$ & $\begin{array}{l}0.502^{* *} \\
{[0.142]}\end{array}$ & $\begin{array}{c}0.444 * * * \\
{[0.140]}\end{array}$ & $\begin{array}{l}0.358^{* *} \\
{[0.162]}\end{array}$ & $\begin{array}{c}0.247 * * * \\
{[0.122]}\end{array}$ & $\begin{array}{c}0.425^{* * *} \\
{[0.138]}\end{array}$ & $\begin{array}{c}0.167 * * * \\
{[0.102]}\end{array}$ \\
\hline Age $=18$ & $\begin{array}{c}1.086 \\
{[0.365]}\end{array}$ & $\begin{array}{c}1.047 \\
{[0.352]}\end{array}$ & $\begin{array}{c}1.044 \\
{[0.350]}\end{array}$ & $\begin{array}{c}0.958 \\
{[0.212]}\end{array}$ & $\begin{array}{c}0.956 \\
{[0.212]}\end{array}$ & $\begin{array}{c}1.034 \\
{[0.377]}\end{array}$ & $\begin{array}{c}0.941 \\
{[0.344]}\end{array}$ & $\begin{array}{c}0.946 \\
{[0.211]}\end{array}$ & $\begin{array}{c}0.987 \\
{[0.458]}\end{array}$ \\
\hline Age $=19$ & $\begin{array}{c}1.314 \\
{[0.377]}\end{array}$ & $\begin{array}{c}1.310 \\
{[0.376]}\end{array}$ & $\begin{array}{c}1.309 \\
{[0.374]} \\
\end{array}$ & $\begin{array}{c}1.213 \\
{[0.223]}\end{array}$ & $\begin{array}{c}1.218 \\
{[0.224]}\end{array}$ & $\begin{array}{c}1.267 \\
{[0.390]} \\
\end{array}$ & $\begin{array}{c}1.199 \\
{[0.369]}\end{array}$ & $\begin{array}{c}1.212 \\
{[0.223]}\end{array}$ & $\begin{array}{c}1.247 \\
{[0.487]}\end{array}$ \\
\hline
\end{tabular}

Notes: The data come from the 2013 DHS. The sample includes 48 months on each side of the donut-hole in panel (I) and 84 months on each side of the donut-hole in panel (II). The number of observations is 2,325 in all cells in part (I), while it is 4,066 in all cells for ages up to 18 and 4040 in all cells for age 19 in panel (II). The policy dummy is one when year of birth is greater than 1987. Each cell comes from a separate logistic regression of ever-married status in panel (A) and of ever-given-birth status in panel (B) at the specified age on the policy variable and the specified time trends in month and year of birth; additional control variables include dummies for month of birth. In marriage estimations at age 13 in part (I), two months (February and March) are combined to generate a single dummy; and in fertility esimations at age 15, February and March as well as November and December are combined. Odds ratios and their standard errors are given as estimated parameters. The standard errors are clustered at the month-and-year-of-birth level. Statistical significance is $* * *$ at 1 percent level, $* *$ at 5 percent level, $*$ at 10 percent level. 
Table C5: Policy Effect on Marriage and First-Birth by Age with Dummies for Month of Birth - 10-Year Intervals around the Donut-hole (Sample B3), Odds ratios

\begin{tabular}{|c|c|c|c|c|c|c|c|c|c|}
\hline \multirow[b]{2}{*}{$\begin{array}{l}\text { Degree of } \\
\text { Polynomial }\end{array}$} & \multicolumn{6}{|c|}{ Single Time Trend } & \multicolumn{3}{|c|}{ Split Time Trends } \\
\hline & $\begin{array}{l}\text { One } \\
(1)\end{array}$ & $\begin{array}{l}\text { Two } \\
(2)\end{array}$ & $\begin{array}{l}\text { Three } \\
\text { (3) }\end{array}$ & $\begin{array}{l}\text { Four } \\
\text { (4) }\end{array}$ & $\begin{array}{c}\text { Five } \\
(5)\end{array}$ & $\begin{array}{l}\text { Six } \\
(6) \\
\end{array}$ & $\begin{array}{l}\text { One } \\
(7)\end{array}$ & $\begin{array}{c}\text { Two } \\
(8)\end{array}$ & $\begin{array}{l}\text { Three } \\
(9)\end{array}$ \\
\hline \multicolumn{10}{|c|}{ A) Dependent Variable: Ever Married } \\
\hline Age $=13$ & $\begin{array}{c}0.832 \\
{[0.539]}\end{array}$ & $\begin{array}{c}1.140 \\
{[0.735]}\end{array}$ & $\begin{array}{c}1.212 \\
{[1.074]}\end{array}$ & $\begin{array}{c}1.283 \\
{[1.149]}\end{array}$ & $\begin{array}{l}1.146 \\
{[1.370]}\end{array}$ & $\begin{array}{c}0.727 \\
{[0.963]}\end{array}$ & $\begin{array}{c}1.077 \\
{[0.672]}\end{array}$ & $\begin{array}{c}0.954 \\
{[1.180]}\end{array}$ & $\begin{array}{c}0.162 \\
{[0.414]}\end{array}$ \\
\hline Age $=14$ & $\begin{array}{c}0.622 \\
{[0.317]}\end{array}$ & $\begin{array}{c}0.850 \\
{[0.424]}\end{array}$ & $\begin{array}{c}0.783 \\
{[0.543]}\end{array}$ & $\begin{array}{c}0.568 \\
{[0.436]}\end{array}$ & $\begin{array}{c}0.703 \\
{[0.689]}\end{array}$ & $\begin{array}{c}0.360 \\
{[0.399]}\end{array}$ & $\begin{array}{c}0.780 \\
{[0.384]}\end{array}$ & $\begin{array}{c}0.386 \\
{[0.388]}\end{array}$ & $\begin{array}{c}0.155 \\
{[0.282]}\end{array}$ \\
\hline Age $=15$ & $\begin{array}{l}0.465^{* *} \\
{[0.139]}\end{array}$ & $\begin{array}{l}0.584 * \\
{[0.185]}\end{array}$ & $\begin{array}{l}0.496^{*} \\
{[0.211]}\end{array}$ & $\begin{array}{l}0.343^{* *} \\
{[0.175]}\end{array}$ & $\begin{array}{l}0.301 * \\
{[0.185]}\end{array}$ & $\begin{array}{l}0.214^{* *} \\
{[0.150]}\end{array}$ & $\begin{array}{l}0.550^{*} \\
{[0.174]}\end{array}$ & $\begin{array}{c}0.240 * * \\
{[0.152]}\end{array}$ & $\begin{array}{l}0.097^{* *} \\
{[0.112]}\end{array}$ \\
\hline Age $=16$ & $\begin{array}{c}0.737 \\
{[0.143]}\end{array}$ & $\begin{array}{c}0.840 \\
{[0.168]}\end{array}$ & $\begin{array}{l}0.540^{* *} \\
{[0.156]}\end{array}$ & $\begin{array}{c}0.362 * * * \\
{[0.111]}\end{array}$ & $\begin{array}{l}0.451^{* *} \\
{[0.180]}\end{array}$ & & $\begin{array}{c}0.804 \\
{[0.162]}\end{array}$ & $\begin{array}{c}0.279 * * * \\
{[0.103]}\end{array}$ & $\begin{array}{c}0.485 \\
{[0.306]}\end{array}$ \\
\hline Age $=17$ & $\begin{array}{c}1.204 \\
{[0.191]}\end{array}$ & $\begin{array}{l}1.335^{*} \\
{[0.214]}\end{array}$ & $\begin{array}{c}1.210 \\
{[0.300]}\end{array}$ & $\begin{array}{c}0.843 \\
{[0.215]}\end{array}$ & $\begin{array}{c}1.144 \\
{[0.404]}\end{array}$ & $\begin{array}{c}1.155 \\
{[0.424]}\end{array}$ & $\begin{array}{c}1.269 \\
{[0.202]}\end{array}$ & $\begin{array}{c}0.817 \\
{[0.255]}\end{array}$ & $\begin{array}{c}1.545 \\
{[0.906]}\end{array}$ \\
\hline Age $=18$ & $\begin{array}{c}1.228 \\
{[0.163]}\end{array}$ & $\begin{array}{l}1.325^{* *} \\
{[0.188]}\end{array}$ & $\begin{array}{c}1.183 \\
{[0.249]}\end{array}$ & $\begin{array}{c}0.794 \\
{[0.177]}\end{array}$ & $\begin{array}{c}1.012 \\
{[0.291]}\end{array}$ & $\begin{array}{l}1.520 \\
{[0.504]}\end{array}$ & $\begin{array}{l}1.274 * \\
{[0.177]}\end{array}$ & $\begin{array}{c}0.789 \\
{[0.207]}\end{array}$ & $\begin{array}{c}1.382 \\
{[0.691]}\end{array}$ \\
\hline Age $=19$ & $\begin{array}{c}1.138 \\
{[0.147]}\end{array}$ & $\begin{array}{c}1.146 \\
{[0.169]}\end{array}$ & $\begin{array}{c}1.118 \\
{[0.223]}\end{array}$ & $\begin{array}{c}0.930 \\
{[0.233]}\end{array}$ & $\begin{array}{c}1.092 \\
{[0.306]}\end{array}$ & $\begin{array}{c}1.459 \\
{[0.524]}\end{array}$ & $\begin{array}{c}1.126 \\
{[0.157]} \\
\end{array}$ & $\begin{array}{c}0.894 \\
{[0.253]} \\
\end{array}$ & $\begin{array}{c}1.444 \\
{[0.774]}\end{array}$ \\
\hline \multicolumn{10}{|c|}{ B) Dependent Variable: Ever Given Birth } \\
\hline Age $=15$ & $\begin{array}{c}0.747 \\
{[0.386]}\end{array}$ & $\begin{array}{c}0.749 \\
{[0.385]}\end{array}$ & $\begin{array}{c}0.531 \\
{[0.402]}\end{array}$ & $\begin{array}{c}0.274 \\
{[0.256]}\end{array}$ & $\begin{array}{c}0.880 \\
{[1.125]}\end{array}$ & $\begin{array}{c}0.342 \\
{[0.554]}\end{array}$ & $\begin{array}{c}0.697 \\
{[0.370]}\end{array}$ & $\begin{array}{c}0.159 \\
{[0.231]}\end{array}$ & $\begin{array}{c}1.245 \\
{[3.067]}\end{array}$ \\
\hline Age $=16$ & $\begin{array}{c}0.674 \\
{[0.230]}\end{array}$ & $\begin{array}{c}0.755 \\
{[0.264]}\end{array}$ & $\begin{array}{c}0.547 \\
{[0.251]}\end{array}$ & $\begin{array}{l}0.265^{* *} \\
{[0.147]}\end{array}$ & $\begin{array}{c}0.418 \\
{[0.289]}\end{array}$ & $\begin{array}{l}0.255^{*} \\
{[0.196]}\end{array}$ & $\begin{array}{c}0.697 \\
{[0.250]}\end{array}$ & $\begin{array}{l}0.146^{* *} \\
{[0.111]}\end{array}$ & $\begin{array}{c}0.156 \\
{[0.217]}\end{array}$ \\
\hline Age $=17$ & $\begin{array}{c}0.763 \\
{[0.173]}\end{array}$ & $\begin{array}{c}0.731 \\
{[0.192]}\end{array}$ & $\begin{array}{l}0.472^{* *} \\
{[0.158]}\end{array}$ & $\begin{array}{c}0.234 * * * \\
{[0.099]}\end{array}$ & $\begin{array}{l}0.299 * * \\
{[0.143]}\end{array}$ & $\begin{array}{c}0.210^{* * *} \\
{[0.117]}\end{array}$ & $\begin{array}{c}0.700 \\
{[0.186]}\end{array}$ & $\begin{array}{c}0.137 * * * \\
{[0.071]}\end{array}$ & $\begin{array}{l}0.095^{* *} \\
{[0.088]}\end{array}$ \\
\hline Age $=18$ & $\begin{array}{c}1.104 \\
{[0.206]}\end{array}$ & $\begin{array}{c}1.245 \\
{[0.247]}\end{array}$ & $\begin{array}{c}1.062 \\
{[0.297]}\end{array}$ & $\begin{array}{c}0.615 \\
{[0.196]}\end{array}$ & $\begin{array}{c}0.928 \\
{[0.370]}\end{array}$ & $\begin{array}{c}1.304 \\
{[0.624]}\end{array}$ & $\begin{array}{c}1.170 \\
{[0.228]}\end{array}$ & $\begin{array}{c}0.557 \\
{[0.209]}\end{array}$ & $\begin{array}{c}1.544 \\
{[1.105]}\end{array}$ \\
\hline Age $=19$ & $\begin{array}{c}1.200 \\
{[0.180]}\end{array}$ & $\begin{array}{c}1.238 \\
{[0.211]}\end{array}$ & $\begin{array}{c}1.373 \\
{[0.307]}\end{array}$ & $\begin{array}{c}1.067 \\
{[0.310]}\end{array}$ & $\begin{array}{c}1.125 \\
{[0.364]}\end{array}$ & $\begin{array}{c}1.873 \\
{[0.816]}\end{array}$ & $\begin{array}{c}1.196 \\
{[0.197]}\end{array}$ & $\begin{array}{c}1.133 \\
{[0.369]} \\
\end{array}$ & $\begin{array}{c}1.315 \\
{[0.855]}\end{array}$ \\
\hline
\end{tabular}

Notes: The data come from the 2013 DHS. The sample includes 10 years on each side of the donut-hole. The number of observations is 5,946 in all cells for all ages up to 15, 5,921 for age 16, 5,589 for age 17, 5,273 for age 18, and 4,970 for age 19 . The policy dummy is one when year of birth is greater than 1987. Each cell comes from a separate logistic regression of evermarried status in panel (A) and of ever-given-birth status in panel (B) at the specified age on the policy variable and the specified time trends in month and year of birth; additional control variables include dummies for month of birth. Odds ratios and their standard errors are given as estimated parameters. The standard errors are clustered at the month-and-year-of-birth level. Statistical significance is *** at 1 percent level, $* *$ at 5 percent level, ${ }^{*}$ at 10 percent level. 
Table C6: Policy Effect on Marriage and First-Birth Hazard Rates with Dummies for Month

of Birth - 4-Year and 7-Year Intervals around the Donut-hole (Samples B1 and B2), Odds

ratios

\begin{tabular}{|c|c|c|c|c|c|c|c|c|c|}
\hline \multirow[b]{3}{*}{$\begin{array}{l}\text { Degree of } \\
\text { Polynomial }\end{array}$} & \multicolumn{3}{|c|}{ I) 4-Year Intervals } & \multicolumn{6}{|c|}{ II) 7-Year Intervals } \\
\hline & \multicolumn{2}{|c|}{$\begin{array}{c}\text { Single Time } \\
\text { Trend }\end{array}$} & \multirow{2}{*}{$\begin{array}{c}\begin{array}{c}\text { Split } \\
\text { Trends }\end{array} \\
\text { One } \\
(3)\end{array}$} & \multicolumn{4}{|c|}{ Single Time Trend } & \multicolumn{2}{|c|}{ Split Time Trends } \\
\hline & $\begin{array}{l}\text { One } \\
(1)\end{array}$ & $\begin{array}{l}\text { Two } \\
\text { (2) }\end{array}$ & & $\begin{array}{l}\text { One } \\
(4)\end{array}$ & $\begin{array}{l}\text { Two } \\
(5)\end{array}$ & $\begin{array}{c}\text { Three } \\
(6)\end{array}$ & $\begin{array}{l}\text { Four } \\
(7)\end{array}$ & $\begin{array}{l}\text { One } \\
(8)\end{array}$ & $\begin{array}{l}\text { Two } \\
\text { (9) }\end{array}$ \\
\hline \multicolumn{10}{|c|}{ A) Dependent Variable: Marriage status conditional on not being married until that age } \\
\hline Age $=14$ & $\begin{array}{c}0.308 \\
{[0.395]}\end{array}$ & $\begin{array}{c}0.215 \\
{[0.297]}\end{array}$ & $\begin{array}{c}0.227 \\
{[0.311]}\end{array}$ & $\begin{array}{c}0.703 \\
{[0.576]}\end{array}$ & $\begin{array}{c}0.561 \\
{[0.494]}\end{array}$ & $\begin{array}{c}0.283 \\
{[0.377]}\end{array}$ & $\begin{array}{c}0.119 \\
{[0.192]}\end{array}$ & $\begin{array}{c}0.563 \\
{[0.490]}\end{array}$ & $\begin{array}{c}0.086 \\
{[0.170]}\end{array}$ \\
\hline Age $=15$ & $\begin{array}{l}0.319^{*} \\
{[0.191]}\end{array}$ & $\begin{array}{c}0.360 \\
{[0.250]}\end{array}$ & $\begin{array}{c}0.318 \\
{[0.226]}\end{array}$ & $\begin{array}{c}0.316^{* * * *} \\
{[0.130]}\end{array}$ & $\begin{array}{c}0.272 * * * \\
{[0.138]}\end{array}$ & $\begin{array}{l}0.263 * \\
{[0.186]}\end{array}$ & $\begin{array}{l}0.244^{*} \\
{[0.193]}\end{array}$ & $\begin{array}{l}0.263 * * \\
{[0.138]}\end{array}$ & $\begin{array}{c}0.183 * \\
{[0.189]}\end{array}$ \\
\hline Age $=16$ & $\begin{array}{c}0.862 \\
{[0.388]}\end{array}$ & $\begin{array}{c}0.828 \\
{[0.385]}\end{array}$ & $\begin{array}{c}0.827 \\
{[0.386]}\end{array}$ & $\begin{array}{c}0.652 \\
{[0.193]}\end{array}$ & $\begin{array}{c}0.636 \\
{[0.194]}\end{array}$ & $\begin{array}{c}0.897 \\
{[0.444]}\end{array}$ & $\begin{array}{c}0.850 \\
{[0.448]}\end{array}$ & $\begin{array}{c}0.631 \\
{[0.195]}\end{array}$ & $\begin{array}{c}0.941 \\
{[0.631]}\end{array}$ \\
\hline Age $=17$ & $\begin{array}{c}4.433 * * * \\
{[1.751]}\end{array}$ & $\begin{array}{c}5.087 * * * \\
{[2.081]}\end{array}$ & $\begin{array}{c}5.437 * * * \\
{[2.323]}\end{array}$ & $\begin{array}{c}2.479^{* * * *} \\
{[0.682]}\end{array}$ & $\begin{array}{c}2.494 * * * \\
{[0.696]}\end{array}$ & $\begin{array}{c}3.691 * * * \\
{[1.552]}\end{array}$ & $\begin{array}{c}4.213 * * * \\
{[1.914]}\end{array}$ & $\begin{array}{c}2.546 * * * \\
{[0.725]}\end{array}$ & $\begin{array}{c}6.639 * * * \\
{[4.193]}\end{array}$ \\
\hline Age $=18$ & $\begin{array}{c}0.836 \\
{[0.290]}\end{array}$ & $\begin{array}{c}0.836 \\
{[0.290]}\end{array}$ & $\begin{array}{c}0.834 \\
{[0.289]}\end{array}$ & $\begin{array}{c}1.116 \\
{[0.278]}\end{array}$ & $\begin{array}{c}1.114 \\
{[0.279]}\end{array}$ & $\begin{array}{c}1.138 \\
{[0.438]}\end{array}$ & $\begin{array}{c}1.148 \\
{[0.432]}\end{array}$ & $\begin{array}{c}1.115 \\
{[0.279]}\end{array}$ & $\begin{array}{c}1.021 \\
{[0.480]}\end{array}$ \\
\hline Age $=19$ & $\begin{array}{c}1.135 \\
{[0.475]}\end{array}$ & $\begin{array}{c}1.127 \\
{[0.484]}\end{array}$ & $\begin{array}{c}1.124 \\
{[0.483]}\end{array}$ & $\begin{array}{c}1.214 \\
{[0.355]}\end{array}$ & $\begin{array}{c}1.276 \\
{[0.356]}\end{array}$ & $\begin{array}{c}0.672 \\
{[0.311]}\end{array}$ & $\begin{array}{c}0.621 \\
{[0.306]}\end{array}$ & $\begin{array}{c}1.255 \\
{[0.349]}\end{array}$ & $\begin{array}{c}0.602 \\
{[0.388]}\end{array}$ \\
\hline \multicolumn{10}{|c|}{ B) Dependent Variable: Birth status conditional on not giving birth until that age } \\
\hline Age $=15$ & $\begin{array}{c}0.209 \\
{[0.250]}\end{array}$ & $\begin{array}{l}0.055^{*} \\
{[0.086]}\end{array}$ & $\begin{array}{l}0.056^{*} \\
{[0.086]}\end{array}$ & $\begin{array}{l}0.284 * \\
{[0.198]}\end{array}$ & $\begin{array}{l}0.143 * * \\
{[0.120]}\end{array}$ & $\begin{array}{c}0.199 \\
{[0.281]}\end{array}$ & $\begin{array}{c}0.074 \\
{[0.119]}\end{array}$ & $\begin{array}{l}0.118^{* *} \\
{[0.110]}\end{array}$ & $\begin{array}{c}0.036 \\
{[0.075]}\end{array}$ \\
\hline Age $=16$ & $\begin{array}{l}0.316^{*} \\
{[0.209]}\end{array}$ & $\begin{array}{l}0.204^{*} \\
{[0.174]}\end{array}$ & $\begin{array}{l}0.175^{*} \\
{[0.159]}\end{array}$ & $\begin{array}{c}0.529 \\
{[0.273]}\end{array}$ & $\begin{array}{c}0.434 \\
{[0.275]}\end{array}$ & $\begin{array}{c}0.291 \\
{[0.220]}\end{array}$ & $\begin{array}{l}0.199^{*} \\
{[0.179]}\end{array}$ & $\begin{array}{c}0.412 \\
{[0.270]}\end{array}$ & $\begin{array}{l}0.087 * * \\
{[0.107]}\end{array}$ \\
\hline Age $=17$ & $\begin{array}{c}0.546 \\
{[0.302]}\end{array}$ & $\begin{array}{c}0.477 \\
{[0.276]}\end{array}$ & $\begin{array}{c}0.454 \\
{[0.267]}\end{array}$ & $\begin{array}{l}0.470 * * \\
{[0.173]}\end{array}$ & $\begin{array}{l}0.453^{* *} \\
{[0.176]}\end{array}$ & $\begin{array}{l}0.327^{*} \\
{[0.193]}\end{array}$ & $\begin{array}{l}0.248^{* *} \\
{[0.162]}\end{array}$ & $\begin{array}{l}0.440 * * \\
{[0.177]}\end{array}$ & $\begin{array}{l}0.167^{* *} \\
{[0.136]}\end{array}$ \\
\hline Age $=18$ & $\begin{array}{l}2.476^{* *} \\
{[1.123]}\end{array}$ & $\begin{array}{l}2.503 * * \\
{[1.146]}\end{array}$ & $\begin{array}{l}2.545^{* *} \\
{[1.186]}\end{array}$ & $\begin{array}{l}2.007 * * \\
{[0.604]}\end{array}$ & $\begin{array}{l}1.995^{* *} \\
{[0.574]}\end{array}$ & $\begin{array}{l}2.701 * * \\
{[1.282]}\end{array}$ & $\begin{array}{l}2.742 * * \\
{[1.341]}\end{array}$ & $\begin{array}{l}1.973 * * \\
{[0.572]}\end{array}$ & $\begin{array}{l}3.735^{* *} \\
{[2.377]}\end{array}$ \\
\hline Age $=19$ & $\begin{array}{c}1.753 \\
{[0.715]}\end{array}$ & $\begin{array}{c}1.759 \\
{[0.702]}\end{array}$ & $\begin{array}{c}1.740 \\
{[0.689]}\end{array}$ & $\begin{array}{l}1.754 * * \\
{[0.486]}\end{array}$ & $\begin{array}{l}1.786^{* *} \\
{[0.479]}\end{array}$ & $\begin{array}{c}1.721 \\
{[0.774]}\end{array}$ & $\begin{array}{c}1.743 \\
{[0.772]}\end{array}$ & $\begin{array}{l}1.776 * * \\
{[0.473]}\end{array}$ & $\begin{array}{c}1.705 \\
{[0.935]}\end{array}$ \\
\hline
\end{tabular}

Notes: The data come from the 2013 DHS. The sample includes 48 months on each side of the donut-hole in panel (I) and 84 months on each side of the donut-hole in panel (II). The samples in panel (A) are restricted to individuals aged 14 and above, while the samples in panel (B) are restricted to individuals aged 15 and above. The number of observations is 12,533 in all columns in panel (A) of part (I), while it is 11,055 in all columns in panel (B) of part (I). In part (II), the number of observations is 21,769 in all columns in panel (A) and 19,221 in all columns in panel (B). The policy dummy is one when year of birth is greater than 1987. The estimates in each column come from a separate logistic regression of marriage hazard status in panel (A) and of birth hazard status in panel (B) on the interactions of the policy variable with each age dummy and the interactions of the specified time trends in month and year of birth with each age dummy. Other covariates included are month-of-birth dummies. Odds ratios and their standard errors are given as estimated parameters. The standard errors are clustered at the month-year of birth level. Statistical significance is *** at 1 percent level, ** at 5 percent level, * at 10 percent level. 
Table C7: Policy Effect on Marriage and First-Birth Hazard Rates with Dummies for Month of Birth - 10-Year Intervals around the Donut-hole (Sample B3), Odds ratios

\begin{tabular}{|c|c|c|c|c|c|c|c|c|c|}
\hline \multirow[b]{2}{*}{$\begin{array}{l}\text { Degree of } \\
\text { Polynomial }\end{array}$} & \multicolumn{6}{|c|}{ Single Time Trend } & \multicolumn{3}{|c|}{ Split Time Trends } \\
\hline & $\begin{array}{c}\text { One } \\
(1)\end{array}$ & $\begin{array}{l}\text { Two } \\
(2)\end{array}$ & $\begin{array}{c}\text { Three } \\
\text { (3) }\end{array}$ & $\begin{array}{c}\text { Four } \\
(4)\end{array}$ & $\begin{array}{c}\text { Five } \\
(5)\end{array}$ & $\begin{array}{l}\text { Six } \\
(6) \\
\end{array}$ & $\begin{array}{l}\text { One } \\
(7)\end{array}$ & $\begin{array}{c}\text { Two } \\
(8)\end{array}$ & $\begin{array}{c}\text { Three } \\
(9)\end{array}$ \\
\hline \multicolumn{10}{|c|}{ A) Dependent Variable: Marriage status conditional on not being married until that age } \\
\hline Age $=14$ & $\begin{array}{c}0.495 \\
{[0.332]}\end{array}$ & $\begin{array}{c}0.675 \\
{[0.447]}\end{array}$ & $\begin{array}{c}0.590 \\
{[0.574]}\end{array}$ & $\begin{array}{c}0.253 \\
{[0.299]}\end{array}$ & $\begin{array}{c}0.420 \\
{[0.650]}\end{array}$ & $\begin{array}{c}0.224 \\
{[0.404]}\end{array}$ & $\begin{array}{c}0.601 \\
{[0.388]}\end{array}$ & $\begin{array}{c}0.169 \\
{[0.262]}\end{array}$ & $\begin{array}{c}0.278 \\
{[0.687]}\end{array}$ \\
\hline Age $=15$ & $\begin{array}{c}0.370 * * * \\
{[0.134]}\end{array}$ & $\begin{array}{l}0.452^{* *} \\
{[0.177]}\end{array}$ & $\begin{array}{c}0.363^{* *} \\
{[0.178]}\end{array}$ & $\begin{array}{l}0.236^{* *} \\
{[0.154]}\end{array}$ & $\begin{array}{l}0.162 * * \\
{[0.121]}\end{array}$ & $\begin{array}{l}0.140^{* *} \\
{[0.124]}\end{array}$ & $\begin{array}{c}0.430^{* *} \\
{[0.170]}\end{array}$ & $\begin{array}{l}0.166^{* *} \\
{[0.137]}\end{array}$ & $\begin{array}{l}0.063 * \\
{[0.094]}\end{array}$ \\
\hline Age $=16$ & $\begin{array}{c}1.133 \\
{[0.290]}\end{array}$ & $\begin{array}{c}1.206 \\
{[0.298]}\end{array}$ & $\begin{array}{c}0.573 \\
{[0.212]}\end{array}$ & $\begin{array}{l}0.404 * * \\
{[0.165]}\end{array}$ & $\begin{array}{c}0.728 \\
{[0.426]}\end{array}$ & $\begin{array}{c}0.887 \\
{[0.493]}\end{array}$ & $\begin{array}{c}1.173 \\
{[0.290]}\end{array}$ & $\begin{array}{c}0.324^{* *} \\
{[0.169]}\end{array}$ & $\begin{array}{c}1.411 \\
{[1.265]}\end{array}$ \\
\hline Age $=17$ & $\begin{array}{c}2.614 * * * \\
{[0.635]}\end{array}$ & $\begin{array}{c}2.827 * * * \\
{[0.655]}\end{array}$ & $\begin{array}{c}3.043 * * * \\
{[1.040]}\end{array}$ & $\begin{array}{l}2.320 * * \\
{[0.843]}\end{array}$ & $\begin{array}{c}4.336^{* * *} \\
{[2.100]}\end{array}$ & $\begin{array}{c}4.477 * * * \\
{[2.196]}\end{array}$ & $\begin{array}{c}2.679 * * * \\
{[0.626]}\end{array}$ & $\begin{array}{l}2.865 * * 1 \\
{[1.327]}\end{array}$ & $\begin{array}{c}10.833^{* * *} \\
{[9.509]}\end{array}$ \\
\hline Age $=18$ & $\begin{array}{c}1.329 \\
{[0.273]}\end{array}$ & $\begin{array}{c}1.427 \\
{[0.311]}\end{array}$ & $\begin{array}{c}1.048 \\
{[0.305]}\end{array}$ & $\begin{array}{c}0.843 \\
{[0.285]}\end{array}$ & $\begin{array}{c}0.815 \\
{[0.316]}\end{array}$ & $\begin{array}{c}1.275 \\
{[0.636]}\end{array}$ & $\begin{array}{c}1.403 \\
{[0.295]}\end{array}$ & $\begin{array}{c}0.786 \\
{[0.307]}\end{array}$ & $\begin{array}{c}0.733 \\
{[0.528]}\end{array}$ \\
\hline Age $=19$ & $\begin{array}{c}1.084 \\
{[0.265]}\end{array}$ & $\begin{array}{c}1.330 \\
{[0.354]}\end{array}$ & $\begin{array}{c}0.881 \\
{[0.302]}\end{array}$ & $\begin{array}{c}0.657 \\
{[0.307]}\end{array}$ & $\begin{array}{c}1.007 \\
{[0.514]}\end{array}$ & $\begin{array}{c}2.097 \\
{[1.462]}\end{array}$ & $\begin{array}{c}1.277 \\
{[0.322]}\end{array}$ & $\begin{array}{c}0.553 \\
{[0.324]}\end{array}$ & $\begin{array}{l}7.408 * * \\
{[6.800]}\end{array}$ \\
\hline \multicolumn{10}{|c|}{ B) Dependent Variable: Birth status conditional on not giving birth until that age } \\
\hline Age $=15$ & $\begin{array}{c}0.618 \\
{[0.379]}\end{array}$ & $\begin{array}{c}0.492 \\
{[0.301]}\end{array}$ & $\begin{array}{c}0.182 * \\
{[0.161]}\end{array}$ & $\begin{array}{c}0.072 * * \\
{[0.076]}\end{array}$ & $\begin{array}{c}0.231 \\
{[0.345]}\end{array}$ & $\begin{array}{c}0.042^{*} \\
{[0.075]}\end{array}$ & $\begin{array}{c}0.466 \\
{[0.296]}\end{array}$ & $\begin{array}{l}0.013^{* *} \\
{[0.024]}\end{array}$ & $\begin{array}{c}0.013 \\
{[0.035]}\end{array}$ \\
\hline Age $=16$ & $\begin{array}{c}0.564 \\
{[0.257]}\end{array}$ & $\begin{array}{c}0.698 \\
{[0.337]}\end{array}$ & $\begin{array}{c}0.557 \\
{[0.328]}\end{array}$ & $\begin{array}{l}0.243^{*} \\
{[0.187]}\end{array}$ & $\begin{array}{l}0.218^{*} \\
{[0.186]}\end{array}$ & $\begin{array}{l}0.184^{*} \\
{[0.168]}\end{array}$ & $\begin{array}{c}0.631 \\
{[0.311]}\end{array}$ & $\begin{array}{l}0.132^{* *} \\
{[0.132]}\end{array}$ & $\begin{array}{c}0.029 * * \\
{[0.049]}\end{array}$ \\
\hline Age $=17$ & $\begin{array}{c}0.894 \\
{[0.285]}\end{array}$ & $\begin{array}{c}0.861 \\
{[0.294]}\end{array}$ & $\begin{array}{l}0.388 * * \\
{[0.169]}\end{array}$ & $\begin{array}{c}0.198 * * * \\
{[0.111]}\end{array}$ & $\begin{array}{l}0.235^{* *} \\
{[0.154]}\end{array}$ & $\begin{array}{l}0.261^{*} \\
{[0.185]}\end{array}$ & $\begin{array}{c}0.844 \\
{[0.289]}\end{array}$ & $\begin{array}{c}0.114 * * * \\
{[0.079]}\end{array}$ & $\begin{array}{l}0.105^{*} \\
{[0.122]}\end{array}$ \\
\hline Age $=18$ & $\begin{array}{l}1.845^{* *} \\
{[0.502]}\end{array}$ & $\begin{array}{c}2.407 * * * \\
{[0.605]}\end{array}$ & $\begin{array}{c}2.318^{* *} \\
{[0.892]}\end{array}$ & $\begin{array}{c}1.729 \\
{[0.734]}\end{array}$ & $\begin{array}{l}2.848^{*} \\
{[1.625]}\end{array}$ & $\begin{array}{c}4.950^{* * *} \\
{[3.092]}\end{array}$ & $\begin{array}{c}2.158^{* * *} \\
{[0.529]}\end{array}$ & $\begin{array}{c}1.919 \\
{[0.992]}\end{array}$ & $\begin{array}{l}9.260 * * \\
{[8.659]}\end{array}$ \\
\hline Age $=19$ & $\begin{array}{l}1.513^{*} \\
{[0.365]}\end{array}$ & $\begin{array}{l}1.782 * * \\
{[0.433]}\end{array}$ & $\begin{array}{l}1.970^{* *} \\
{[0.675]}\end{array}$ & $\begin{array}{c}1.765 \\
{[0.742]}\end{array}$ & $\begin{array}{c}1.473 \\
{[0.696]}\end{array}$ & $\begin{array}{c}2.712 \\
{[1.669]}\end{array}$ & $\begin{array}{l}1.704 * * \\
{[0.399]}\end{array}$ & $\begin{array}{c}2.101 \\
{[0.965]}\end{array}$ & $\begin{array}{c}1.566 \\
{[1.309]}\end{array}$ \\
\hline
\end{tabular}

Notes: The data come from the 2013 DHS. The sample includes 10 years on each side of the donut-hole. The samples in panel (A) are restricted to individuals aged 14 and above, while the samples in panel (B) are restricted to individuals aged 15 and above. The number of observations is 30,151 in all columns in panel (A), while it is 26,220 in all columns in panel (B). The policy dummy is one when year of birth is greater than 1987. The estimates in each column come from a separate logistic regression of marriage hazard status in panel (A) and of birth hazard status in panel (B) on the interactions of the policy variable with each age dummy and the interactions of the specified time trends in month and year of birth with each age dummy. Other covariates included are month-ofbirth dummies. Odds ratios and their standard errors are given as estimated parameters. The standard errors are clustered at the month-and-year-of-birth level. Statistical significance is *** at 1 percent level, ** at 5 percent level, * at 10 percent level. 
Table C8: Policy Effect on Marriage and First-Birth by Age with Dummies for Birth-Month, NUTS-1 Regions, and Rural Area - Samples (B1) and (B2), Odds ratios

\begin{tabular}{|c|c|c|c|c|c|c|c|c|c|}
\hline \multirow{3}{*}{$\begin{array}{l}\text { Degree of } \\
\text { Polynomial } \\
\end{array}$} & \multicolumn{3}{|c|}{ I) 4-Year Intervals } & \multicolumn{6}{|c|}{ II) 7-Year Intervals } \\
\hline & \multicolumn{2}{|c|}{$\begin{array}{c}\text { Single Time } \\
\text { Trend }\end{array}$} & \multirow{2}{*}{$\begin{array}{c}\begin{array}{c}\text { Split } \\
\text { Trends }\end{array} \\
\begin{array}{c}\text { One } \\
(3)\end{array}\end{array}$} & \multicolumn{4}{|c|}{ Single Time Trend } & \multicolumn{2}{|c|}{ Split Time Trends } \\
\hline & $\begin{array}{l}\text { One } \\
(1)\end{array}$ & $\begin{array}{l}\text { Two } \\
(2)\end{array}$ & & $\begin{array}{l}\text { One } \\
(4)\end{array}$ & $\begin{array}{l}\text { Two } \\
(5)\end{array}$ & $\begin{array}{c}\text { Three } \\
(6)\end{array}$ & $\begin{array}{c}\text { Four } \\
(7) \\
\end{array}$ & $\begin{array}{l}\text { One } \\
(8)\end{array}$ & $\begin{array}{l}\text { Two } \\
(9) \\
\end{array}$ \\
\hline \multicolumn{10}{|c|}{ A) Dependent Variable: Ever Married } \\
\hline Age $=13$ & $\begin{array}{c}0.774 \\
{[0.836]}\end{array}$ & $\begin{array}{c}0.699 \\
{[0.793]}\end{array}$ & $\begin{array}{c}0.662 \\
{[0.780]}\end{array}$ & $\begin{array}{c}1.180 \\
{[0.909]}\end{array}$ & $\begin{array}{c}1.449 \\
{[1.067]}\end{array}$ & $\begin{array}{c}0.938 \\
{[1.062]}\end{array}$ & $\begin{array}{c}0.450 \\
{[0.626]}\end{array}$ & $\begin{array}{c}1.345 \\
{[0.993]}\end{array}$ & $\begin{array}{c}0.271 \\
{[0.537]}\end{array}$ \\
\hline Age $=14$ & $\begin{array}{c}0.389 \\
{[0.357]}\end{array}$ & $\begin{array}{c}0.305 \\
{[0.296]}\end{array}$ & $\begin{array}{c}0.297 \\
{[0.291]}\end{array}$ & $\begin{array}{c}0.872 \\
{[0.513]}\end{array}$ & $\begin{array}{c}0.856 \\
{[0.525]}\end{array}$ & $\begin{array}{c}0.463 \\
{[0.397]}\end{array}$ & $\begin{array}{c}0.208 \\
{[0.223]}\end{array}$ & $\begin{array}{c}0.822 \\
{[0.508]}\end{array}$ & $\begin{array}{c}0.130 \\
{[0.179]}\end{array}$ \\
\hline Age $=15$ & $\begin{array}{c}0.299 * * \\
{[0.150]}\end{array}$ & $\begin{array}{c}0.284 * * \\
{[0.157]}\end{array}$ & $\begin{array}{c}0.268 * * \\
{[0.149]}\end{array}$ & $\begin{array}{c}0.461 * * \\
{[0.165]}\end{array}$ & $\begin{array}{c}0.419 * * \\
{[0.169]}\end{array}$ & $\begin{array}{c}0.305 * * \\
{[0.177]}\end{array}$ & $\begin{array}{c}0.244 * * \\
{[0.153]}\end{array}$ & $\begin{array}{c}0.409 * * \\
{[0.167]}\end{array}$ & $\begin{array}{c}0.169 * * \\
{[0.135]}\end{array}$ \\
\hline Age $=16$ & $\begin{array}{c}0.533 * * \\
{[0.159]}\end{array}$ & $\begin{array}{c}0.507 * * \\
{[0.164]}\end{array}$ & $\begin{array}{c}0.497 * * \\
{[0.158]}\end{array}$ & $\begin{array}{c}0.528 * * * \\
{[0.120]}\end{array}$ & $\begin{array}{c}0.491 * * * \\
{[0.119]}\end{array}$ & $\begin{array}{c}0.520^{*} \\
{[0.175]}\end{array}$ & $\begin{array}{c}0.453 * * \\
{[0.154]}\end{array}$ & $\begin{array}{c}0.483 * * * \\
{[0.118]}\end{array}$ & $\begin{array}{c}0.437 * * \\
{[0.179]}\end{array}$ \\
\hline Age $=17$ & $\begin{array}{c}1.457 \\
{[0.430]}\end{array}$ & $\begin{array}{c}1.412 \\
{[0.416]}\end{array}$ & $\begin{array}{c}1.417 \\
{[0.415]}\end{array}$ & $\begin{array}{c}1.045 \\
{[0.198]}\end{array}$ & $\begin{array}{c}1.027 \\
{[0.195]}\end{array}$ & $\begin{array}{c}1.295 \\
{[0.408]}\end{array}$ & $\begin{array}{c}1.204 \\
{[0.378]}\end{array}$ & $\begin{array}{c}1.021 \\
{[0.193]}\end{array}$ & $\begin{array}{c}1.368 \\
{[0.555]}\end{array}$ \\
\hline Age $=18$ & $\begin{array}{c}1.170 \\
{[0.276]}\end{array}$ & $\begin{array}{c}1.162 \\
{[0.272]}\end{array}$ & $\begin{array}{c}1.162 \\
{[0.270]}\end{array}$ & $\begin{array}{c}1.069 \\
{[0.170]}\end{array}$ & $\begin{array}{c}1.058 \\
{[0.166]}\end{array}$ & $\begin{array}{c}1.253 \\
{[0.323]}\end{array}$ & $\begin{array}{c}1.235 \\
{[0.315]}\end{array}$ & $\begin{array}{c}1.059 \\
{[0.166]}\end{array}$ & $\begin{array}{c}1.279 \\
{[0.412]}\end{array}$ \\
\hline Age $=19$ & $\begin{array}{c}1.265 \\
{[0.308]}\end{array}$ & $\begin{array}{c}1.256 \\
{[0.305]} \\
\end{array}$ & $\begin{array}{c}1.256 \\
{[0.303]} \\
\end{array}$ & $\begin{array}{c}1.142 \\
{[0.183]}\end{array}$ & $\begin{array}{c}1.143 \\
{[0.184]} \\
\end{array}$ & $\begin{array}{c}1.047 \\
{[0.273]}\end{array}$ & $\begin{array}{c}1.020 \\
{[0.266]}\end{array}$ & $\begin{array}{c}1.140 \\
{[0.182]}\end{array}$ & $\begin{array}{c}1.044 \\
{[0.347]} \\
\end{array}$ \\
\hline \multicolumn{10}{|c|}{ B) Dependent Variable: Ever Given Birth } \\
\hline Age $=15$ & $\begin{array}{c}0.343 \\
{[0.363]}\end{array}$ & $\begin{array}{c}0.221 \\
{[0.304]}\end{array}$ & $\begin{array}{c}0.265 \\
{[0.348]}\end{array}$ & $\begin{array}{c}0.592 \\
{[0.352]}\end{array}$ & $\begin{array}{c}0.364 \\
{[0.261]}\end{array}$ & $\begin{array}{c}0.605 \\
{[0.701]}\end{array}$ & $\begin{array}{c}0.368 \\
{[0.518]}\end{array}$ & $\begin{array}{c}0.345 \\
{[0.263]}\end{array}$ & $\begin{array}{c}0.466 \\
{[0.802]}\end{array}$ \\
\hline Age $=16$ & $\begin{array}{c}0.312 * * \\
{[0.173]}\end{array}$ & $\begin{array}{c}0.214 * * \\
{[0.143]}\end{array}$ & $\begin{array}{c}0.206^{* *} \\
{[0.140]}\end{array}$ & $\begin{array}{c}0.556 \\
{[0.215]}\end{array}$ & $\begin{array}{c}0.414^{*} \\
{[0.190]}\end{array}$ & $\begin{array}{c}0.363 \\
{[0.233]}\end{array}$ & $\begin{array}{c}0.260 * \\
{[0.182]}\end{array}$ & $\begin{array}{c}0.396^{*} \\
{[0.190]}\end{array}$ & $\begin{array}{c}0.178 * \\
{[0.166]}\end{array}$ \\
\hline Age $=17$ & $\begin{array}{c}0.382 * * \\
{[0.148]}\end{array}$ & $\begin{array}{c}0.308 * * * \\
{[0.134]}\end{array}$ & $\begin{array}{c}0.295 * * * \\
{[0.128]}\end{array}$ & $\begin{array}{c}0.476 * * * \\
{[0.136]}\end{array}$ & $\begin{array}{c}0.421 * * * \\
{[0.134]}\end{array}$ & $\begin{array}{c}0.327 * * \\
{[0.146]}\end{array}$ & $\begin{array}{c}0.234 * * * \\
{[0.114]}\end{array}$ & $\begin{array}{c}0.404 * * * \\
{[0.134]}\end{array}$ & $\begin{array}{c}0.159 * * * \\
{[0.095]}\end{array}$ \\
\hline Age $=18$ & $\begin{array}{c}1.096 \\
{[0.371]}\end{array}$ & $\begin{array}{c}1.062 \\
{[0.363]}\end{array}$ & $\begin{array}{c}1.058 \\
{[0.361]}\end{array}$ & $\begin{array}{c}0.938 \\
{[0.210]}\end{array}$ & $\begin{array}{c}0.936 \\
{[0.212]}\end{array}$ & $\begin{array}{c}1.005 \\
{[0.368]}\end{array}$ & $\begin{array}{c}0.925 \\
{[0.341]}\end{array}$ & $\begin{array}{c}0.927 \\
{[0.211]}\end{array}$ & $\begin{array}{c}0.972 \\
{[0.453]}\end{array}$ \\
\hline Age $=19$ & $\begin{array}{c}1.360 \\
{[0.386]}\end{array}$ & $\begin{array}{c}1.361 \\
{[0.389]}\end{array}$ & $\begin{array}{c}1.358 \\
{[0.386]}\end{array}$ & $\begin{array}{c}1.204 \\
{[0.223]}\end{array}$ & $\begin{array}{c}1.208 \\
{[0.225]}\end{array}$ & $\begin{array}{c}1.259 \\
{[0.385]}\end{array}$ & $\begin{array}{c}1.205 \\
{[0.370]}\end{array}$ & $\begin{array}{c}1.203 \\
{[0.224]}\end{array}$ & $\begin{array}{c}1.255 \\
{[0.488]}\end{array}$ \\
\hline
\end{tabular}

Notes: The data come from the 2013 DHS. The sample includes 48 months on each side of the donut-hole in panel (I) and 84 months on each side of the donut-hole in panel (II). The number of observations is 2,325 all cells in part (I), while it is 4,066 in all cells for ages up to 18 and 4040 in all cells for age 19 in panel (II). The policy dummy is one when year of birth is greater than 1987. Each cell comes from a separate logistic regression of ever-married status in panel (A) and of ever-given-birth status in panel (B) at the specified age on the policy variable and the specified time trends in month and year of birth; additional control variables include month and place of birth dummies (NUTS-1 level region dummies and an urban/rural dummy). With 48-month intervals, certain months and regions are combined in panel (A) regressions at ages 13 and 14; and with 84-year intervals, certain regions are combined in panel (A) regressions for age 13. With 48-month intervals, certain months and regions are combined in panel (B) regressions at age 15 and certain regions are combined in panel (B) regressions at age 16; with 84-intervals, certain regions are combined in panel (B) regressions at age 15. Odds ratios and their standard errors are given as estimated parameters. The standard errors are clustered at the month-and-year-of-birth level. Statistical significance is *** at 1 percent level, ${ }^{* *}$ at 5 percent level, ${ }^{*}$ at 10 percent level. 
Table C9: Policy Effect on Marriage and First-Birth by Age with Dummies for Birth-Month, NUTS-1 Regions, and Rural Area - Sample (B3), Odds ratios

\begin{tabular}{|c|c|c|c|c|c|c|c|c|c|}
\hline \multirow[b]{2}{*}{$\begin{array}{l}\text { Degree of } \\
\text { Polynomial }\end{array}$} & \multicolumn{6}{|c|}{ Single Time Trend } & \multicolumn{3}{|c|}{ Split Time Trends } \\
\hline & $\begin{array}{l}\text { One } \\
(1)\end{array}$ & $\begin{array}{l}\text { Two } \\
\text { (2) }\end{array}$ & $\begin{array}{l}\text { Three } \\
\text { (3) }\end{array}$ & $\begin{array}{l}\text { Four } \\
\text { (4) }\end{array}$ & $\begin{array}{c}\text { Five } \\
(5)\end{array}$ & $\begin{array}{l}\text { Six } \\
(6)\end{array}$ & $\begin{array}{l}\text { One } \\
(7)\end{array}$ & $\begin{array}{l}\text { Two } \\
(8)\end{array}$ & $\begin{array}{l}\text { Three } \\
\text { (9) }\end{array}$ \\
\hline \multicolumn{10}{|c|}{ A) Dependent Variable: Ever Married } \\
\hline Age $=13$ & $\begin{array}{c}0.777 \\
{[0.515]}\end{array}$ & $\begin{array}{c}1.080 \\
{[0.716]}\end{array}$ & $\begin{array}{c}1.162 \\
{[1.058]}\end{array}$ & $\begin{array}{c}1.314 \\
{[1.197]}\end{array}$ & $\begin{array}{l}1.250 \\
{[1.544]}\end{array}$ & $\begin{array}{c}0.833 \\
{[1.131]}\end{array}$ & $\begin{array}{c}1.029 \\
{[0.658]}\end{array}$ & $\begin{array}{c}1.024 \\
{[1.267]}\end{array}$ & $\begin{array}{c}0.217 \\
{[0.553]}\end{array}$ \\
\hline Age $=14$ & $\begin{array}{c}0.610 \\
{[0.311]}\end{array}$ & $\begin{array}{c}0.815 \\
{[0.410]}\end{array}$ & $\begin{array}{c}0.761 \\
{[0.522]}\end{array}$ & $\begin{array}{c}0.576 \\
{[0.437]}\end{array}$ & $\begin{array}{c}0.705 \\
{[0.682]}\end{array}$ & $\begin{array}{c}0.386 \\
{[0.422]}\end{array}$ & $\begin{array}{c}0.753 \\
{[0.373]}\end{array}$ & $\begin{array}{c}0.407 \\
{[0.400]}\end{array}$ & $\begin{array}{c}0.173 \\
{[0.307]}\end{array}$ \\
\hline Age $=15$ & $\begin{array}{c}0.441^{* * *} \\
{[0.133]}\end{array}$ & $\begin{array}{l}0.543^{*} \\
{[0.174]}\end{array}$ & $\begin{array}{l}0.466^{*} \\
{[0.198]}\end{array}$ & $\begin{array}{c}0.335^{* *} \\
{[0.170]}\end{array}$ & $\begin{array}{c}0.293 * * \\
{[0.180]}\end{array}$ & $\begin{array}{l}0.219^{* *} \\
{[0.153]}\end{array}$ & $\begin{array}{c}0.514^{* *} \\
{[0.165]}\end{array}$ & $\begin{array}{c}0.243 * * \\
{[0.151]}\end{array}$ & $\begin{array}{l}0.104^{* *} \\
{[0.118]}\end{array}$ \\
\hline Age $=16$ & $\begin{array}{l}0.702 * \\
{[0.137]}\end{array}$ & $\begin{array}{c}0.791 \\
{[0.159]}\end{array}$ & $\begin{array}{c}0.506^{* *} \\
{[0.145]}\end{array}$ & $\begin{array}{c}0.347^{* * *} \\
{[0.105]}\end{array}$ & $\begin{array}{l}0.438^{* *} \\
{[0.171]}\end{array}$ & $\begin{array}{l}0.452^{* *} \\
{[0.182]}\end{array}$ & $\begin{array}{c}0.760 \\
{[0.153]}\end{array}$ & $\begin{array}{c}0.272 * * * \\
{[0.099]}\end{array}$ & $\begin{array}{c}0.499 \\
{[0.312]}\end{array}$ \\
\hline Age $=17$ & $\begin{array}{c}1.163 \\
{[0.184]}\end{array}$ & $\begin{array}{c}1.276 \\
{[0.205]}\end{array}$ & $\begin{array}{c}1.194 \\
{[0.293]}\end{array}$ & $\begin{array}{c}0.853 \\
{[0.216]}\end{array}$ & $\begin{array}{c}1.145 \\
{[0.399]}\end{array}$ & $\begin{array}{c}1.186 \\
{[0.436]}\end{array}$ & $\begin{array}{c}1.217 \\
{[0.193]}\end{array}$ & $\begin{array}{c}0.840 \\
{[0.259]}\end{array}$ & $\begin{array}{c}1.547 \\
{[0.915]}\end{array}$ \\
\hline Age $=18$ & $\begin{array}{c}1.194 \\
{[0.159]}\end{array}$ & $\begin{array}{l}1.274^{*} \\
{[0.180]}\end{array}$ & $\begin{array}{c}1.170 \\
{[0.245]}\end{array}$ & $\begin{array}{c}0.814 \\
{[0.180]}\end{array}$ & $\begin{array}{c}1.000 \\
{[0.286]}\end{array}$ & $\begin{array}{c}1.527 \\
{[0.515]}\end{array}$ & $\begin{array}{c}1.229 \\
{[0.171]}\end{array}$ & $\begin{array}{c}0.816 \\
{[0.213]}\end{array}$ & $\begin{array}{c}1.294 \\
{[0.663]}\end{array}$ \\
\hline Age $=19$ & $\begin{array}{c}1.112 \\
{[0.146]}\end{array}$ & $\begin{array}{c}1.124 \\
{[0.169]}\end{array}$ & $\begin{array}{c}1.098 \\
{[0.221]} \\
\end{array}$ & $\begin{array}{c}0.947 \\
{[0.241]} \\
\end{array}$ & $\begin{array}{c}1.096 \\
{[0.311]}\end{array}$ & $\begin{array}{c}1.522 \\
{[0.566]}\end{array}$ & $\begin{array}{c}1.105 \\
{[0.158]} \\
\end{array}$ & $\begin{array}{c}0.908 \\
{[0.265]}\end{array}$ & $\begin{array}{c}1.401 \\
{[0.799]}\end{array}$ \\
\hline \multicolumn{10}{|c|}{ B) Dependent Variable: Ever Given Birth } \\
\hline Age $=15$ & $\begin{array}{c}0.728 \\
{[0.385]}\end{array}$ & $\begin{array}{c}0.706 \\
{[0.372]}\end{array}$ & $\begin{array}{c}0.512 \\
{[0.395]}\end{array}$ & $\begin{array}{c}0.275 \\
{[0.261]}\end{array}$ & $\begin{array}{c}0.869 \\
{[1.131]}\end{array}$ & $\begin{array}{c}0.371 \\
{[0.603]}\end{array}$ & $\begin{array}{c}0.660 \\
{[0.359]}\end{array}$ & $\begin{array}{c}0.171 \\
{[0.245]}\end{array}$ & $\begin{array}{c}1.315 \\
{[3.174]}\end{array}$ \\
\hline Age $=16$ & $\begin{array}{c}0.648 \\
{[0.225]}\end{array}$ & $\begin{array}{c}0.710 \\
{[0.253]}\end{array}$ & $\begin{array}{c}0.527 \\
{[0.244]}\end{array}$ & $\begin{array}{l}0.265^{* *} \\
{[0.149]}\end{array}$ & $\begin{array}{c}0.411 \\
{[0.288]}\end{array}$ & $\begin{array}{l}0.268^{*} \\
{[0.207]}\end{array}$ & $\begin{array}{c}0.657 \\
{[0.240]}\end{array}$ & $\begin{array}{l}0.153^{* *} \\
{[0.117]}\end{array}$ & $\begin{array}{c}0.169 \\
{[0.231]}\end{array}$ \\
\hline Age $=17$ & $\begin{array}{c}0.726 \\
{[0.168]}\end{array}$ & $\begin{array}{c}0.679 \\
{[0.183]}\end{array}$ & $\begin{array}{l}0.445^{* *} \\
{[0.149]}\end{array}$ & $\begin{array}{c}0.230^{* * *} \\
{[0.098]}\end{array}$ & $\begin{array}{c}0.281 * * * \\
{[0.136]}\end{array}$ & $\begin{array}{c}0.209 * * * \\
{[0.117]}\end{array}$ & $\begin{array}{c}0.654 \\
{[0.178]}\end{array}$ & $\begin{array}{c}0.140 * * * \\
{[0.073]}\end{array}$ & $\begin{array}{l}0.093 * * \\
{[0.086]}\end{array}$ \\
\hline Age $=18$ & $\begin{array}{c}1.061 \\
{[0.202]}\end{array}$ & $\begin{array}{c}1.185 \\
{[0.241]}\end{array}$ & $\begin{array}{c}1.037 \\
{[0.293]}\end{array}$ & $\begin{array}{c}0.624 \\
{[0.205]}\end{array}$ & $\begin{array}{c}0.906 \\
{[0.367]}\end{array}$ & $\begin{array}{c}1.274 \\
{[0.629]}\end{array}$ & $\begin{array}{c}1.117 \\
{[0.223]}\end{array}$ & $\begin{array}{c}0.574 \\
{[0.220]}\end{array}$ & $\begin{array}{c}1.402 \\
{[1.036]}\end{array}$ \\
\hline Age $=19$ & $\begin{array}{c}1.181 \\
{[0.180]}\end{array}$ & $\begin{array}{c}1.218 \\
{[0.212]}\end{array}$ & $\begin{array}{c}1.362 \\
{[0.307]}\end{array}$ & $\begin{array}{c}1.081 \\
{[0.320]} \\
\end{array}$ & $\begin{array}{c}1.103 \\
{[0.363]}\end{array}$ & $\begin{array}{c}1.905 \\
{[0.848]}\end{array}$ & $\begin{array}{c}1.179 \\
{[0.198]}\end{array}$ & $\begin{array}{c}1.157 \\
{[0.387]}\end{array}$ & $\begin{array}{c}1.224 \\
{[0.823]} \\
\end{array}$ \\
\hline
\end{tabular}

Notes: The data come from the 2013 DHS. The sample includes 10 years on each side of the donut-hole. The number of observations is 5,946 in all cells for all ages up to $15,5,921$ for age 16, 5,589 for age 17, 5,273 for age 18, and 4,970 for age 19 . The policy dummy is one when year of birth is greater than 1987. Each cell comes from a separate logistic regression of evermarried status in panel (A) and of ever-given-birth status in panel (B) at the specified age on the policy variable and the specified time trends in month and year of birth; additional control variables include month and place of birth dummies (NUTS-1 level region dummies and a rural/urban dummy). In marriage estimations at age 13, some regions are combined. Odds ratios and their standard errors are given as estimated parameters. The standard errors are clustered at the month-and-year-of-birth level. Statistical significance is *** at 1 percent level, $* *$ at 5 percent level, $*$ at 10 percent level. 
Table C10: Policy Effect on Marriage and First-Birth Hazard Rates with Birth-Month, NUTS-1 Region, and Rural Dummies - Samples (B1) and (B2), Odds ratios

\begin{tabular}{|c|c|c|c|c|c|c|c|c|c|}
\hline \multirow[b]{3}{*}{$\begin{array}{l}\text { Degree of } \\
\text { Polynomial } \\
\end{array}$} & \multicolumn{3}{|c|}{ I) 4-Year Intervals } & \multicolumn{6}{|c|}{ II) 7-Year Intervals } \\
\hline & \multicolumn{2}{|c|}{$\begin{array}{l}\text { Single Time } \\
\text { Trend }\end{array}$} & \multirow{2}{*}{$\begin{array}{c}\begin{array}{c}\text { Split } \\
\text { Trends }\end{array} \\
\begin{array}{c}\text { One } \\
(3)\end{array}\end{array}$} & \multicolumn{4}{|c|}{ Single Time Trend } & \multicolumn{2}{|c|}{ Split Time Trends } \\
\hline & $\begin{array}{c}\text { One } \\
(1)\end{array}$ & $\begin{array}{l}\text { Two } \\
(2)\end{array}$ & & $\begin{array}{l}\text { One } \\
(4)\end{array}$ & $\begin{array}{l}\text { Two } \\
(5)\end{array}$ & $\begin{array}{c}\text { Three } \\
(6) \\
\end{array}$ & $\begin{array}{c}\text { Four } \\
(7)\end{array}$ & $\begin{array}{c}\text { One } \\
(8)\end{array}$ & $\begin{array}{l}\text { Two } \\
(9)\end{array}$ \\
\hline \multicolumn{10}{|c|}{ A) Dependent Variable: Marriage status conditional on not being married until that age } \\
\hline Age $=14$ & $\begin{array}{c}0.316 \\
{[0.402]}\end{array}$ & $\begin{array}{c}0.224 \\
{[0.309]}\end{array}$ & $\begin{array}{c}0.236 \\
{[0.322]}\end{array}$ & $\begin{array}{c}0.700 \\
{[0.574]}\end{array}$ & $\begin{array}{c}0.556 \\
{[0.493]}\end{array}$ & $\begin{array}{c}0.273 \\
{[0.360]}\end{array}$ & $\begin{array}{c}0.122 \\
{[0.195]}\end{array}$ & $\begin{array}{c}0.561 \\
{[0.491]}\end{array}$ & $\begin{array}{c}0.087 \\
{[0.170]}\end{array}$ \\
\hline Age $=15$ & $\begin{array}{l}0.324 * \\
{[0.195]}\end{array}$ & $\begin{array}{c}0.372 \\
{[0.257]}\end{array}$ & $\begin{array}{c}0.327 \\
{[0.231]}\end{array}$ & $\begin{array}{c}0.311^{* * *} \\
{[0.129]}\end{array}$ & $\begin{array}{c}0.267 * * * \\
{[0.136]}\end{array}$ & $\begin{array}{l}0.254 * \\
{[0.180]}\end{array}$ & $\begin{array}{l}0.244^{*} \\
{[0.191]}\end{array}$ & $\begin{array}{c}0.260^{* * *} \\
{[0.136]}\end{array}$ & $\begin{array}{l}0.184^{*} \\
{[0.187]}\end{array}$ \\
\hline Age $=16$ & $\begin{array}{c}0.880 \\
{[0.390]}\end{array}$ & $\begin{array}{c}0.849 \\
{[0.390]}\end{array}$ & $\begin{array}{c}0.847 \\
{[0.390]}\end{array}$ & $\begin{array}{c}0.629 \\
{[0.185]}\end{array}$ & $\begin{array}{c}0.614 \\
{[0.186]}\end{array}$ & $\begin{array}{c}0.876 \\
{[0.425]}\end{array}$ & $\begin{array}{c}0.838 \\
{[0.432]}\end{array}$ & $\begin{array}{c}0.610 \\
{[0.188]}\end{array}$ & $\begin{array}{c}0.938 \\
{[0.614]}\end{array}$ \\
\hline Age $=17$ & $\begin{array}{c}4.625^{* * *} \\
{[1.823]}\end{array}$ & $\begin{array}{l}5.273^{* * *} \\
{[2.143]}\end{array}$ & $\begin{array}{c}5.638 * * * \\
{[2.386]}\end{array}$ & $\begin{array}{c}2.483 * * * \\
{[0.685]}\end{array}$ & $\begin{array}{c}2.500^{* * * *} \\
{[0.700]}\end{array}$ & $\begin{array}{c}3.710^{* * * *} \\
{[1.566]}\end{array}$ & $\begin{array}{c}4.217 * * * \\
{[1.910]}\end{array}$ & $\begin{array}{c}2.552^{* * *} \\
{[0.727]}\end{array}$ & $\begin{array}{c}6.654^{* * *} \\
{[4.159]}\end{array}$ \\
\hline Age $=18$ & $\begin{array}{c}0.867 \\
{[0.297]}\end{array}$ & $\begin{array}{c}0.867 \\
{[0.297]}\end{array}$ & $\begin{array}{c}0.865 \\
{[0.296]}\end{array}$ & $\begin{array}{c}1.106 \\
{[0.277]}\end{array}$ & $\begin{array}{c}1.105 \\
{[0.279]}\end{array}$ & $\begin{array}{c}1.131 \\
{[0.435]}\end{array}$ & $\begin{array}{c}1.140 \\
{[0.429]}\end{array}$ & $\begin{array}{c}1.106 \\
{[0.278]}\end{array}$ & $\begin{array}{c}1.021 \\
{[0.484]}\end{array}$ \\
\hline Age $=19$ & $\begin{array}{c}1.195 \\
{[0.504]}\end{array}$ & $\begin{array}{c}1.188 \\
{[0.513]}\end{array}$ & $\begin{array}{c}1.183 \\
{[0.512]}\end{array}$ & & $\begin{array}{c}1.271 \\
{[0.360]}\end{array}$ & $\begin{array}{c}0.669 \\
{[0.313]}\end{array}$ & $\begin{array}{c}0.629 \\
{[0.310]}\end{array}$ & $\begin{array}{c}1.251 \\
{[0.354]}\end{array}$ & $\begin{array}{c}0.615 \\
{[0.396]} \\
\end{array}$ \\
\hline \multicolumn{10}{|c|}{ B) Dependent Variable: Birth status conditional on not giving birth until that age } \\
\hline Age $=15$ & $\begin{array}{c}0.207 \\
{[0.247]}\end{array}$ & $\begin{array}{l}0.056^{*} \\
{[0.088]}\end{array}$ & $\begin{array}{l}0.058^{* *} \\
{[0.088]}\end{array}$ & $\begin{array}{l}0.277^{*} \\
{[0.195]}\end{array}$ & $\begin{array}{l}0.138^{*} \\
{[0.117]}\end{array}$ & $\begin{array}{c}0.188 \\
{[0.264]}\end{array}$ & $\begin{array}{c}0.072 \\
{[0.116]}\end{array}$ & $\begin{array}{l}0.115^{* *} \\
{[0.108]}\end{array}$ & $\begin{array}{c}0.036 \\
{[0.073]}\end{array}$ \\
\hline Age $=16$ & $\begin{array}{l}0.313^{*} \\
{[0.209]}\end{array}$ & $\begin{array}{l}0.206^{*} \\
{[0.177]}\end{array}$ & $\begin{array}{l}0.178^{* *} \\
{[0.162]}\end{array}$ & $\begin{array}{c}0.518 \\
{[0.267]}\end{array}$ & $\begin{array}{c}0.423 \\
{[0.270]}\end{array}$ & $\begin{array}{l}0.277^{*} \\
{[0.210]}\end{array}$ & $\begin{array}{l}0.196^{*} \\
{[0.177]}\end{array}$ & $\begin{array}{c}0.403 \\
{[0.266]}\end{array}$ & $\begin{array}{l}0.086^{* *} \\
{[0.106]}\end{array}$ \\
\hline Age $=17$ & $\begin{array}{c}0.543 \\
{[0.297]}\end{array}$ & $\begin{array}{c}0.480 \\
{[0.276]}\end{array}$ & $\begin{array}{c}0.457 \\
{[0.266]}\end{array}$ & $\begin{array}{c}0.457^{* *} \\
{[0.168]}\end{array}$ & $\begin{array}{c}0.441^{* *} \\
{[0.172]}\end{array}$ & $\begin{array}{c}0.313^{* *} \\
{[0.182]}\end{array}$ & $\begin{array}{c}0.241^{* *} \\
{[0.155]}\end{array}$ & $\begin{array}{c}0.429^{* *} \\
{[0.172]}\end{array}$ & $\begin{array}{c}0.163^{* *} \\
{[0.131]}\end{array}$ \\
\hline Age $=18$ & $\begin{array}{c}2.529 * * \\
{[1.156]}\end{array}$ & $\begin{array}{c}2.543 * * \\
{[1.171]}\end{array}$ & $\begin{array}{c}2.584^{* *} \\
{[1.210]}\end{array}$ & $\begin{array}{l}1.982^{* *} \\
{[0.605]}\end{array}$ & $\begin{array}{l}1.968^{* *} \\
{[0.575]}\end{array}$ & $\begin{array}{c}2.628^{* *} \\
{[1.263]}\end{array}$ & $\begin{array}{c}2.665^{* *} \\
{[1.315]}\end{array}$ & $\begin{array}{l}1.947^{* *} \\
{[0.573]}\end{array}$ & $\begin{array}{c}3.599 * * \\
{[2.305]}\end{array}$ \\
\hline Age $=19$ & $\begin{array}{c}1.778 \\
{[0.722]}\end{array}$ & $\begin{array}{c}1.782 \\
{[0.706]}\end{array}$ & $\begin{array}{c}1.760 \\
{[0.692]}\end{array}$ & $\begin{array}{c}1.726^{*} \\
{[0.483]}\end{array}$ & $\begin{array}{l}1.756^{* *} \\
{[0.477]}\end{array}$ & $\begin{array}{c}1.649 \\
{[0.737]}\end{array}$ & $\begin{array}{c}1.684 \\
{[0.738]}\end{array}$ & $\begin{array}{l}1.748^{* *} \\
{[0.470]}\end{array}$ & $\begin{array}{c}1.632 \\
{[0.883]} \\
\end{array}$ \\
\hline
\end{tabular}

Notes: The data come from the 2013 DHS. The sample includes 48 months on each side of the donut-hole in panel (I) and 84 months on each side of the donut-hole in panel (II). The samples in panel (A) are restricted to individuals aged 14 and above, while the samples in panel (B) are restricted to individuals aged 15 and above. The number of observations is 12,533 in all columns in panel (A) of part (I), while it is 11,055 in all columns in panel (B) of part (I). In part (II), the number of observations is 21,769 in all columns in panel (A) and 19,221 in all columns in panel (B). The policy dummy is one when year of birth is greater than 1987. The estimates in each column come from a separate logistic regression of marriage hazard status in panel (A) and of birth hazard status in panel (B) on the interactions of the policy variable with each age dummy and the interactions of the specified time trends in month and year of birth with each age dummy. Other covariates include month dummies, dummies for 13 regions, and a rural/urban dummy. Odds ratios and their standard errors are given as estimated parameters. The standard errors are clustered at the month-and-year-of-birth level. Statistical significance is *** at 1 percent level, ** at 5 percent level, $*$ at 10 percent level. 
Table C11: Policy Effect on Marriage and First-Birth Hazard Rates with Birth-Month, NUTS-1 Region, and Rural Dummies - Sample (B3), Odds ratios

\begin{tabular}{|c|c|c|c|c|c|c|c|c|c|}
\hline \multirow[b]{2}{*}{$\begin{array}{l}\text { Degree of } \\
\text { Polynomial } \\
\end{array}$} & \multicolumn{6}{|c|}{ Single Time Trend } & \multicolumn{3}{|c|}{ Split Time Trends } \\
\hline & $\begin{array}{l}\text { One } \\
(1)\end{array}$ & $\begin{array}{l}\text { Two } \\
(2)\end{array}$ & $\begin{array}{l}\text { Three } \\
\text { (3) }\end{array}$ & $\begin{array}{l}\text { Four } \\
\text { (4) }\end{array}$ & $\begin{array}{c}\text { Five } \\
(5)\end{array}$ & $\begin{array}{l}\text { Six } \\
(6) \\
\end{array}$ & $\begin{array}{l}\text { One } \\
(7)\end{array}$ & $\begin{array}{c}\text { Two } \\
(8)\end{array}$ & $\begin{array}{c}\text { Three } \\
(9)\end{array}$ \\
\hline \multicolumn{10}{|c|}{ A) Dependent Variable: Marriage status conditional on not being married until that age } \\
\hline Age $=14$ & $\begin{array}{c}0.482 \\
{[0.323]}\end{array}$ & $\begin{array}{c}0.648 \\
{[0.432]}\end{array}$ & $\begin{array}{c}0.575 \\
{[0.558]}\end{array}$ & $\begin{array}{c}0.253 \\
{[0.298]}\end{array}$ & $\begin{array}{c}0.418 \\
{[0.642]}\end{array}$ & $\begin{array}{c}0.234 \\
{[0.416]}\end{array}$ & $\begin{array}{c}0.578 \\
{[0.376]}\end{array}$ & $\begin{array}{c}0.173 \\
{[0.266]}\end{array}$ & $\begin{array}{c}0.288 \\
{[0.697]}\end{array}$ \\
\hline Age $=15$ & $\begin{array}{c}0.357^{* * * *} \\
{[0.128]}\end{array}$ & $\begin{array}{l}0.430^{* *} \\
{[0.169]}\end{array}$ & $\begin{array}{c}0.354^{* *} \\
{[0.175]}\end{array}$ & $\begin{array}{c}0.234^{* *} \\
{[0.153]}\end{array}$ & $\begin{array}{l}0.161^{* *} \\
{[0.120]}\end{array}$ & $\begin{array}{l}0.144 * * \\
{[0.126]}\end{array}$ & $\begin{array}{l}0.408^{* *} \\
{[0.163]}\end{array}$ & $\begin{array}{l}0.168^{* *} \\
{[0.139]}\end{array}$ & $\begin{array}{l}0.066^{*} \\
{[0.097]}\end{array}$ \\
\hline Age $=16$ & $\begin{array}{c}1.079 \\
{[0.275]}\end{array}$ & $\begin{array}{c}1.146 \\
{[0.283]}\end{array}$ & $\begin{array}{c}0.553 \\
{[0.203]}\end{array}$ & $\begin{array}{l}0.394 * * \\
{[0.160]}\end{array}$ & $\begin{array}{c}0.709 \\
{[0.410]}\end{array}$ & $\begin{array}{c}0.885 \\
{[0.483]}\end{array}$ & $\begin{array}{c}1.115 \\
{[0.276]}\end{array}$ & $\begin{array}{l}0.321 * * \\
{[0.165]}\end{array}$ & $\begin{array}{c}1.408 \\
{[1.240]}\end{array}$ \\
\hline Age $=17$ & $\begin{array}{c}2.511^{* * * *} \\
{[0.608]}\end{array}$ & $\begin{array}{c}2.720 * * * \\
{[0.628]}\end{array}$ & $\begin{array}{c}3.091^{* * * *} \\
{[1.057]}\end{array}$ & $\begin{array}{c}2.368^{* *} \\
{[0.858]}\end{array}$ & $\begin{array}{c}4.313 * * * \\
{[2.079]}\end{array}$ & $\begin{array}{c}4.521 * * * \\
{[2.217]}\end{array}$ & $\begin{array}{c}2.577 * * * \\
{[0.601]}\end{array}$ & $\begin{array}{c}2.978^{* *} \\
{[1.371]}\end{array}$ & $\begin{array}{c}10.572 * * * \\
{[9.204]}\end{array}$ \\
\hline Age $=18$ & $\begin{array}{c}1.287 \\
{[0.266]}\end{array}$ & $\begin{array}{c}1.376 \\
{[0.302]}\end{array}$ & $\begin{array}{c}1.054 \\
{[0.310]}\end{array}$ & $\begin{array}{c}0.859 \\
{[0.291]}\end{array}$ & $\begin{array}{c}0.809 \\
{[0.317]}\end{array}$ & $\begin{array}{c}1.283 \\
{[0.643]}\end{array}$ & $\begin{array}{c}1.354 \\
{[0.287]}\end{array}$ & $\begin{array}{c}0.811 \\
{[0.317]}\end{array}$ & $\begin{array}{c}0.702 \\
{[0.515]}\end{array}$ \\
\hline Age $=19$ & $\begin{array}{c}1.061 \\
{[0.262]}\end{array}$ & $\begin{array}{c}1.309 \\
{[0.354]}\end{array}$ & $\begin{array}{c}0.878 \\
{[0.304]}\end{array}$ & $\begin{array}{c}0.671 \\
{[0.314]}\end{array}$ & $\begin{array}{c}1.019 \\
{[0.519]}\end{array}$ & $\begin{array}{c}2.265 \\
{[1.567]}\end{array}$ & $\begin{array}{c}1.255 \\
{[0.321]}\end{array}$ & $\begin{array}{c}0.567 \\
{[0.331]}\end{array}$ & $\begin{array}{l}7.297 * * \\
{[6.881]} \\
\end{array}$ \\
\hline \multicolumn{10}{|c|}{ B) Dependent Variable: Birth status conditional on not giving birth until that age } \\
\hline Age $=15$ & $\begin{array}{c}0.601 \\
{[0.371]}\end{array}$ & $\begin{array}{c}0.469 \\
{[0.289]}\end{array}$ & $\begin{array}{c}0.176^{* *} \\
{[0.156]}\end{array}$ & $\begin{array}{c}0.070^{* *} \\
{[0.075]}\end{array}$ & $\begin{array}{c}0.220 \\
{[0.328]}\end{array}$ & $\begin{array}{c}0.042 * \\
{[0.076]}\end{array}$ & $\begin{array}{c}0.444 \\
{[0.285]}\end{array}$ & $\begin{array}{l}0.013 * * \\
{[0.024]}\end{array}$ & $\begin{array}{c}0.013 \\
{[0.037]}\end{array}$ \\
\hline Age $=16$ & $\begin{array}{c}0.546 \\
{[0.248]}\end{array}$ & $\begin{array}{c}0.666 \\
{[0.323]}\end{array}$ & $\begin{array}{c}0.542 \\
{[0.320]}\end{array}$ & $\begin{array}{l}0.239^{*} \\
{[0.185]}\end{array}$ & $\begin{array}{l}0.210^{*} \\
{[0.180]}\end{array}$ & $\begin{array}{l}0.185^{*} \\
{[0.169]}\end{array}$ & $\begin{array}{c}0.604 \\
{[0.299]}\end{array}$ & $\begin{array}{l}0.133 * * \\
{[0.133]}\end{array}$ & $\begin{array}{c}0.030^{* *} \\
{[0.050]}\end{array}$ \\
\hline Age $=17$ & $\begin{array}{c}0.861 \\
{[0.274]}\end{array}$ & $\begin{array}{c}0.818 \\
{[0.281]}\end{array}$ & $\begin{array}{l}0.379 * * \\
{[0.166]}\end{array}$ & $\begin{array}{c}0.197 * * * \\
{[0.110]}\end{array}$ & $\begin{array}{l}0.224^{* *} \\
{[0.146]}\end{array}$ & $\begin{array}{l}0.258^{*} \\
{[0.182]}\end{array}$ & $\begin{array}{c}0.804 \\
{[0.276]}\end{array}$ & $\begin{array}{c}0.116^{* * *} \\
{[0.080]}\end{array}$ & $\begin{array}{l}0.101^{* *} \\
{[0.116]}\end{array}$ \\
\hline Age $=18$ & $\begin{array}{l}1.796 * * \\
{[0.491]}\end{array}$ & $\begin{array}{c}2.336^{* * * *} \\
{[0.595]}\end{array}$ & $\begin{array}{l}2.278^{* *} \\
{[0.886]}\end{array}$ & $\begin{array}{c}1.731 \\
{[0.743]}\end{array}$ & $\begin{array}{l}2.723^{*} \\
{[1.563]}\end{array}$ & $\begin{array}{l}4.760 * * \\
{[2.998]}\end{array}$ & $\begin{array}{c}2.099^{* * *} * \\
{[0.520]}\end{array}$ & $\begin{array}{c}1.921 \\
{[0.999]}\end{array}$ & $\begin{array}{l}8.311^{* *} \\
{[7.839]}\end{array}$ \\
\hline Age $=19$ & $\begin{array}{c}1.490 \\
{[0.362]}\end{array}$ & $\begin{array}{l}1.748 * * \\
{[0.429]}\end{array}$ & $\begin{array}{l}1.929^{*} \\
{[0.665]}\end{array}$ & $\begin{array}{c}1.721 \\
{[0.723]}\end{array}$ & $\begin{array}{c}1.388 \\
{[0.655]}\end{array}$ & $\begin{array}{c}2.610 \\
{[1.603]}\end{array}$ & $\begin{array}{l}1.675^{* *} \\
{[0.395]}\end{array}$ & $\begin{array}{c}2.052 \\
{[0.948]}\end{array}$ & $\begin{array}{c}1.369 \\
{[1.155]} \\
\end{array}$ \\
\hline
\end{tabular}

Notes: The data come from the 2013 DHS. The sample includes 10 years on each side of the donut-hole. The samples in panel (A) are restricted to individuals aged 14 and above, while the samples in panel (B) are restricted to individuals aged 15 and above. The number of observations is 30,151 in all columns in panel (A), while it is 26,220 in all columns in panel (B). The policy dummy is one when year of birth is greater than 1987. The estimates in each column come from a separate logistic regression of marriage hazard status in panel (A) and of birth hazard status in panel (B) on the interactions of the policy variable with each age dummy and the interactions of the specified time trends in month and year of birth with each age dummy. Other covariates include month dummies, dummies for 13 regions, and a rural/urban dummy. Odds ratios and their standard errors are given as estimated parameters. The standard errors are clustered at the month-and-year-of-birth level. Statistical significance is *** at 1 percent level, ${ }^{* *}$ at 5 percent level, * at 10 percent level. 
Table C12: Sensitivity of the Results to the Width of the Donut-hole, Odds ratios

\begin{tabular}{|c|c|c|c|c|c|c|c|c|c|c|}
\hline \multirow[t]{3}{*}{ Donut-hole } & \multicolumn{2}{|c|}{ \pm 6 months } & \multicolumn{2}{|c|}{ \pm 9 months } & \multicolumn{2}{|c|}{ \pm 12 months } & \multicolumn{2}{|c|}{ \pm 15 months } & \multicolumn{2}{|c|}{ \pm 18 months } \\
\hline & $\begin{array}{c}\text { Single } \\
\text { Quadratic } \\
\text { Trend } \\
\end{array}$ & $\begin{array}{c}\text { Split } \\
\text { Linear } \\
\text { Trends }\end{array}$ & $\begin{array}{c}\text { Single } \\
\text { Quadratic } \\
\text { Trend } \\
\end{array}$ & $\begin{array}{c}\text { Split } \\
\text { Linear } \\
\text { Trends }\end{array}$ & $\begin{array}{c}\text { Single } \\
\text { Quadratic } \\
\text { Trend } \\
\end{array}$ & $\begin{array}{c}\text { Split } \\
\text { Linear } \\
\text { Trends }\end{array}$ & $\begin{array}{c}\text { Single } \\
\text { Quadratic } \\
\text { Trend } \\
\end{array}$ & $\begin{array}{c}\text { Split } \\
\text { Linear } \\
\text { Trends } \\
\end{array}$ & $\begin{array}{c}\text { Single } \\
\text { Quadratic } \\
\text { Trend } \\
\end{array}$ & $\begin{array}{c}\text { Split } \\
\text { Linear } \\
\text { Trends } \\
\end{array}$ \\
\hline & $(1)$ & $(2)$ & (3) & (4) & $(5)$ & (6) & $(7)$ & $(8)$ & $(9)$ & $(10)$ \\
\hline \multicolumn{11}{|c|}{ A) Dependent Variable: Ever Married } \\
\hline Age $=13$ & $\begin{array}{c}1.131 \\
{[0.727]}\end{array}$ & $\begin{array}{c}1.048 \\
{[0.684]}\end{array}$ & $\begin{array}{c}1.060 \\
{[0.713]}\end{array}$ & $\begin{array}{c}0.953 \\
{[0.661]}\end{array}$ & $\begin{array}{c}1.066 \\
{[0.774]}\end{array}$ & $\begin{array}{c}0.971 \\
{[0.708]}\end{array}$ & $\begin{array}{c}1.579 \\
{[1.241]}\end{array}$ & $\begin{array}{c}1.424 \\
{[1.111]}\end{array}$ & $\begin{array}{c}1.222 \\
{[1.047]}\end{array}$ & $\begin{array}{c}1.101 \\
{[0.927]}\end{array}$ \\
\hline Age $=14$ & $\begin{array}{c}0.917 \\
{[0.459]}\end{array}$ & $\begin{array}{c}0.911 \\
{[0.451]}\end{array}$ & $\begin{array}{c}0.600 \\
{[0.327]}\end{array}$ & $\begin{array}{c}0.576 \\
{[0.315]}\end{array}$ & $\begin{array}{c}0.775 \\
{[0.457]}\end{array}$ & $\begin{array}{c}0.742 \\
{[0.436]}\end{array}$ & $\begin{array}{c}0.890 \\
{[0.590]}\end{array}$ & $\begin{array}{c}0.842 \\
{[0.555]}\end{array}$ & $\begin{array}{c}0.777 \\
{[0.570]}\end{array}$ & $\begin{array}{c}0.754 \\
{[0.542]}\end{array}$ \\
\hline Age $=15$ & $\begin{array}{c}0.696 \\
{[0.287]}\end{array}$ & $\begin{array}{c}0.694 \\
{[0.294]}\end{array}$ & $\begin{array}{c}0.423^{* *} \\
{[0.150]}\end{array}$ & $\begin{array}{c}0.409 * * \\
{[0.148]}\end{array}$ & $\begin{array}{c}0.406^{* *} \\
{[0.163]}\end{array}$ & $\begin{array}{c}0.395 * * \\
{[0.161]}\end{array}$ & $\begin{array}{c}0.443 * \\
{[0.191]}\end{array}$ & $\begin{array}{c}0.437^{*} \\
{[0.192]}\end{array}$ & $\begin{array}{c}0.440 * \\
{[0.205]}\end{array}$ & $\begin{array}{c}0.448^{*} \\
{[0.210]}\end{array}$ \\
\hline Age $=16$ & $\begin{array}{c}0.756 \\
{[0.179]}\end{array}$ & $\begin{array}{c}0.754 \\
{[0.182]}\end{array}$ & $\begin{array}{c}0.552 * * * \\
{[0.121]}\end{array}$ & $\begin{array}{c}0.546^{* * *} \\
{[0.122]}\end{array}$ & $\begin{array}{l}0.500 * * * \\
{[0.125]}\end{array}$ & $\begin{array}{l}0.492 * * * \\
{[0.124]}\end{array}$ & $\begin{array}{c}0.576^{* *} \\
{[0.160]}\end{array}$ & $\begin{array}{c}0.565^{* *} \\
{[0.159]}\end{array}$ & $\begin{array}{c}0.511 * * \\
{[0.152]}\end{array}$ & $\begin{array}{c}0.506 * * \\
{[0.153]}\end{array}$ \\
\hline Age $=17$ & $\begin{array}{c}1.243 \\
{[0.216]}\end{array}$ & $\begin{array}{c}1.240 \\
{[0.217]}\end{array}$ & $\begin{array}{c}1.005 \\
{[0.173]}\end{array}$ & $\begin{array}{c}0.999 \\
{[0.173]}\end{array}$ & $\begin{array}{c}1.026 \\
{[0.199]}\end{array}$ & $\begin{array}{c}1.021 \\
{[0.198]}\end{array}$ & $\begin{array}{c}1.114 \\
{[0.236]}\end{array}$ & $\begin{array}{c}1.103 \\
{[0.234]}\end{array}$ & $\begin{array}{c}0.963 \\
{[0.212]}\end{array}$ & $\begin{array}{c}0.953 \\
{[0.211]}\end{array}$ \\
\hline Age $=18$ & $\begin{array}{c}1.100 \\
{[0.159]}\end{array}$ & $\begin{array}{c}1.100 \\
{[0.159]}\end{array}$ & $\begin{array}{c}0.989 \\
{[0.154]}\end{array}$ & $\begin{array}{c}0.988 \\
{[0.154]}\end{array}$ & $\begin{array}{c}1.061 \\
{[0.181]}\end{array}$ & $\begin{array}{c}1.061 \\
{[0.182]}\end{array}$ & $\begin{array}{c}1.175 \\
{[0.217]}\end{array}$ & $\begin{array}{c}1.172 \\
{[0.217]}\end{array}$ & $\begin{array}{c}1.161 \\
{[0.226]}\end{array}$ & $\begin{array}{c}1.160 \\
{[0.226]}\end{array}$ \\
\hline Age $=19$ & $\begin{array}{c}1.214 \\
{[0.173]}\end{array}$ & $\begin{array}{c}1.211 \\
{[0.173]}\end{array}$ & $\begin{array}{c}1.089 \\
{[0.165]}\end{array}$ & $\begin{array}{c}1.086 \\
{[0.165]}\end{array}$ & $\begin{array}{c}1.133 \\
{[0.195]} \\
\end{array}$ & $\begin{array}{c}1.130 \\
{[0.194]}\end{array}$ & $\begin{array}{c}1.195 \\
{[0.220]}\end{array}$ & $\begin{array}{c}1.189 \\
{[0.218]}\end{array}$ & $\begin{array}{c}1.134 \\
{[0.214]}\end{array}$ & $\begin{array}{c}1.129 \\
{[0.213]}\end{array}$ \\
\hline \multicolumn{11}{|c|}{ B) Dependent Variable: Ever Given Birth } \\
\hline Age $=15$ & $\begin{array}{c}0.894 \\
{[0.678]}\end{array}$ & $\begin{array}{c}0.912 \\
{[0.720]}\end{array}$ & $\begin{array}{c}0.312^{*} \\
{[0.202]}\end{array}$ & $\begin{array}{c}0.287 * \\
{[0.203]}\end{array}$ & $\begin{array}{c}0.330 \\
{[0.228]}\end{array}$ & $\begin{array}{c}0.313 \\
{[0.232]}\end{array}$ & $\begin{array}{c}0.476 \\
{[0.349]}\end{array}$ & $\begin{array}{c}0.450 \\
{[0.354]}\end{array}$ & $\begin{array}{c}0.251 * \\
{[0.196]}\end{array}$ & $\begin{array}{c}0.208^{*} \\
{[0.177]}\end{array}$ \\
\hline Age $=16$ & $\begin{array}{c}0.628 \\
{[0.290]}\end{array}$ & $\begin{array}{c}0.628 \\
{[0.303]}\end{array}$ & $\begin{array}{c}0.371 * * \\
{[0.158]}\end{array}$ & $\begin{array}{c}0.353 * * \\
{[0.158]}\end{array}$ & $\begin{array}{c}0.385^{* *} \\
{[0.182]}\end{array}$ & $\begin{array}{c}0.367 * * \\
{[0.182]}\end{array}$ & $\begin{array}{c}0.486 \\
{[0.241]}\end{array}$ & $\begin{array}{c}0.467 \\
{[0.241]}\end{array}$ & $\begin{array}{c}0.426 \\
{[0.240]}\end{array}$ & $\begin{array}{c}0.405 \\
{[0.237]}\end{array}$ \\
\hline Age $=17$ & $\begin{array}{c}0.673 \\
{[0.222]}\end{array}$ & $\begin{array}{c}0.662 \\
{[0.228]}\end{array}$ & $\begin{array}{c}0.430 * * * \\
{[0.129]}\end{array}$ & $\begin{array}{c}0.409 * * * \\
{[0.128]}\end{array}$ & $\begin{array}{c}0.424 * * * \\
{[0.139]}\end{array}$ & $\begin{array}{c}0.408 * * * \\
{[0.138]}\end{array}$ & $\begin{array}{c}0.531^{*} \\
{[0.179]}\end{array}$ & $\begin{array}{l}0.515^{*} \\
{[0.179]}\end{array}$ & $\begin{array}{c}0.526^{*} \\
{[0.189]}\end{array}$ & $\begin{array}{c}0.515^{*} \\
{[0.190]}\end{array}$ \\
\hline Age $=18$ & $\begin{array}{c}1.169 \\
{[0.233]}\end{array}$ & $\begin{array}{c}1.163 \\
{[0.234]}\end{array}$ & $\begin{array}{c}0.929 \\
{[0.184]}\end{array}$ & $\begin{array}{c}0.920 \\
{[0.184]}\end{array}$ & $\begin{array}{c}0.922 \\
{[0.208]}\end{array}$ & $\begin{array}{c}0.914 \\
{[0.208]}\end{array}$ & $\begin{array}{c}0.950 \\
{[0.239]}\end{array}$ & $\begin{array}{c}0.940 \\
{[0.238]}\end{array}$ & $\begin{array}{c}0.858 \\
{[0.219]}\end{array}$ & $\begin{array}{c}0.852 \\
{[0.219]}\end{array}$ \\
\hline Age $=19$ & $\begin{array}{c}1.271 \\
{[0.204]}\end{array}$ & $\begin{array}{c}1.267 \\
{[0.203]}\end{array}$ & $\begin{array}{c}1.146 \\
{[0.190]}\end{array}$ & $\begin{array}{c}1.140 \\
{[0.190]}\end{array}$ & $\begin{array}{c}1.197 \\
{[0.220]}\end{array}$ & $\begin{array}{c}1.191 \\
{[0.220]}\end{array}$ & $\begin{array}{c}1.304 \\
{[0.256]}\end{array}$ & $\begin{array}{c}1.296 \\
{[0.255]}\end{array}$ & $\begin{array}{c}1.314 \\
{[0.265]}\end{array}$ & $\begin{array}{c}1.308 \\
{[0.264]}\end{array}$ \\
\hline
\end{tabular}

Notes: The data comes from the 2013 DHS. The sample includes the 1979-94 birth cohorts except for those born in the donut-hole specified in the column headings. The estimates with the baseline donut-hole (12 months on each side of the cut-off) are given in columns (5) and (6). The policy dummy is one when year of birth is greater than 1987. Each cell comes from a separate logistic regression of ever-married status in panel (A) and of ever-given-birth status in panel (B) at the specified age on the policy variable as well as the specified time trends in month and year of birth. Odds ratios and their standard errors are given as estimated parameters. The standard errors are clustered at the month-and-year-of-birth level. Statistical significance is *** at 1 percent level, ** at 5 percent level, * at 10 percent level. 
Table C13: Effect of the New Civil Code on Marriage Hazard Rates by Age, Odds ratios

\begin{tabular}{lcccccc}
\hline \multicolumn{5}{c}{ A) Dependent Variable: Marriage status conditional on not being married until that age } \\
\cline { 2 - 7 } Degree of & One & Two & Three & Four & One & Two \\
Polynomial & $(1)$ & $(2)$ & $(3)$ & $(4)$ & $(5)$ & $(6)$ \\
\hline \multicolumn{2}{c}{ II) 2-Year Intervals on both sides of the cut-off } & $(1983-84$ vs. $1985-86$ Birth Cohorts $)$ \\
\hline Age=14 & 0.966 & 0.759 & 0.698 & 0.751 & 29.905 & 3.111 \\
& $(0.100)$ & $(0.221)$ & $(0.213)$ & $(0.389)$ & $(74.238)$ & $(8.361)$ \\
Age=15 & $0.887^{* * *}$ & 0.950 & 0.943 & 1.293 & 1.376 & 3.141 \\
& $(0.031)$ & $(0.128)$ & $(0.125)$ & $(0.313)$ & $(1.031)$ & $(3.099)$ \\
Age=16 & 1.035 & 1.162 & 1.166 & 1.176 & 2.183 & 2.241 \\
& $(0.035)$ & $(0.165)$ & $(0.169)$ & $(0.318)$ & $(2.171)$ & $(3.555)$ \\
Age=17 & $1.100^{* * *}$ & $1.298^{*}$ & $1.294^{*}$ & 1.106 & 0.880 & 2.301 \\
& $(0.034)$ & $(0.182)$ & $(0.183)$ & $(0.304)$ & $(0.879)$ & $(3.443)$ \\
Age=18 & 1.003 & 1.159 & $1.195^{*}$ & $1.348^{*}$ & 0.549 & 1.340 \\
& $(0.030)$ & $(0.104)$ & $(0.113)$ & $(0.203)$ & $(0.408)$ & $(1.260)$ \\
Age=19 & 1.009 & 1.096 & 1.096 & 0.938 & 0.594 & 0.684 \\
& $(0.022)$ & $(0.113)$ & $(0.113)$ & $(0.193)$ & $(0.390)$ & $(0.594)$ \\
\hline
\end{tabular}

II) 4-Year Interval before the cut-off, 2-Year after (1981-84 vs. 1985-86 Birth Cohorts)

\begin{tabular}{lcccccc}
\hline Age=14 & 1.013 & 0.905 & 0.966 & 0.942 & 23.130 & 18.042 \\
& $(0.053)$ & $(0.120)$ & $(0.243)$ & $(0.256)$ & $(44.209)$ & $(47.796)$ \\
Age=15 & $0.927 * * *$ & $0.891 * *$ & 0.857 & 0.858 & 2.375 & 2.316 \\
& $(0.025)$ & $(0.045)$ & $(0.098)$ & $(0.100)$ & $(1.629)$ & $(2.074)$ \\
Age=16 & 0.991 & 1.040 & 1.206 & 1.232 & 0.970 & 1.038 \\
& $(0.021)$ & $(0.056)$ & $(0.154)$ & $(0.158)$ & $(0.808)$ & $(1.392)$ \\
Age=17 & 1.018 & $1.176^{* * *}$ & $1.267 * *$ & $1.312^{* *}$ & 0.252 & 0.781 \\
& $(0.020)$ & $(0.059)$ & $(0.140)$ & $(0.155)$ & $(0.220)$ & $(0.895)$ \\
Age=18 & 1.018 & 0.984 & 1.022 & 1.022 & 0.933 & 0.462 \\
& $(0.016)$ & $(0.038)$ & $(0.076)$ & $(0.077)$ & $(0.550)$ & $(0.365)$ \\
Age=19 & $0.973 *$ & 1.061 & 1.091 & 1.118 & 0.384 & 0.578 \\
& $(0.015)$ & $(0.042)$ & $(0.084)$ & $(0.094)$ & $(0.231)$ & $(0.414)$ \\
\hline
\end{tabular}

Notes: The data come from the 2013 DHS. The sample includes 24 months on each side of the cut-off. The number of observations is 6,327 in all columns in panel (A) and 9,641 in all columns in panel (B). The estimates in each column come from a separate logistic regression of marriage hazard status on the interactions of the intensity of the exposure to the new civil code with each age dummy and the interactions of the specified time trends in month and year of birth with each age dummy, dummies for each birth-month, dummies for the NUTS-1 level region of birth, and a dummy for rural region of birth. Odds ratios and their standard errors are given as estimated parameters. The standard errors are clustered at the month-and-year-of-birth level. Statistical significance is *** at 1 percent level, ${ }^{* *}$ at 5 percent level, * at 10 percent level. 